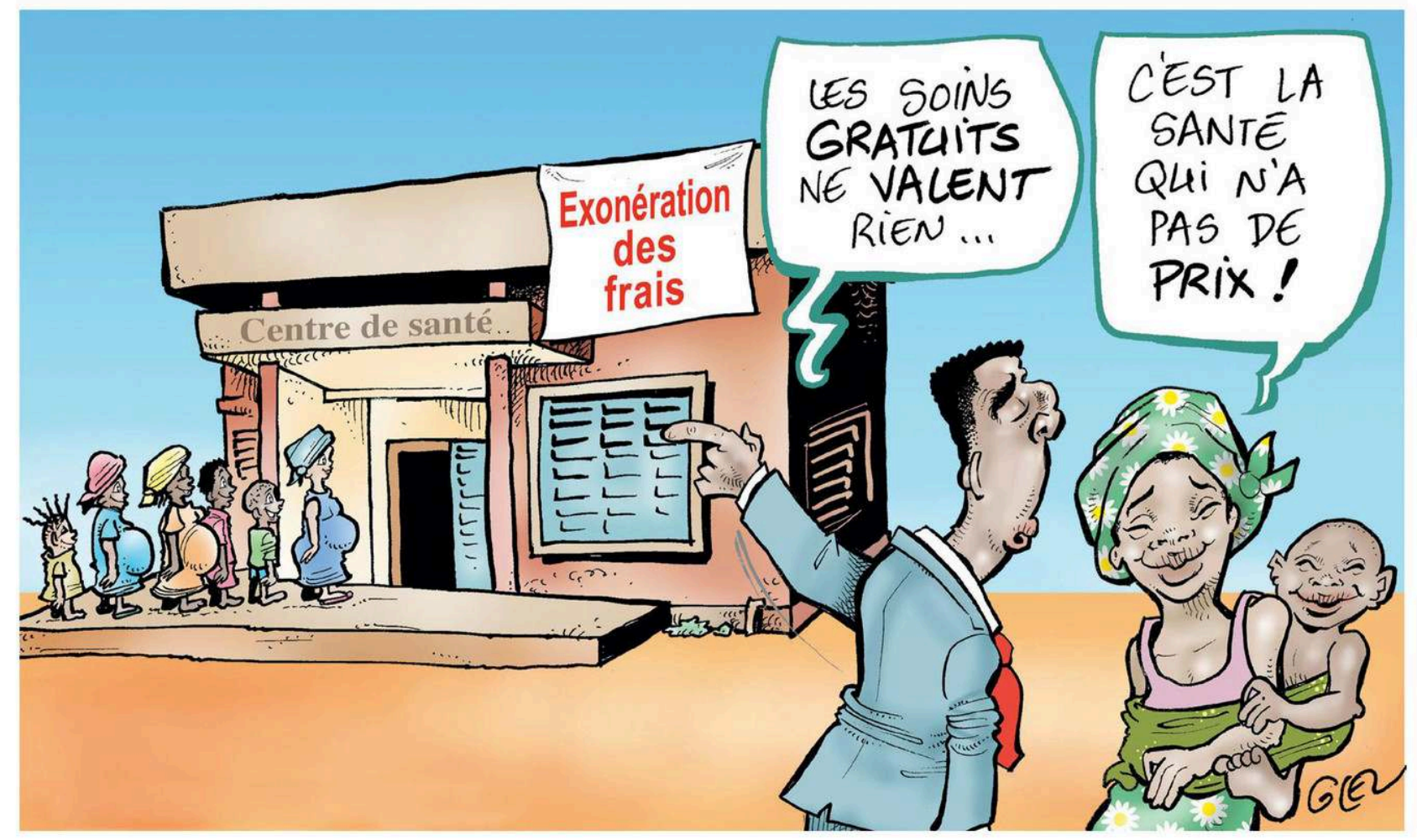

\title{
Des idées reçues en santé mondiale
}

Sous la direction de

Valéry Ridde et Fatoumata Ouattara

\section{LIBRE ACCÈS}

Projet pilote réalisé

en collaboration avec

la Direction des

bibliothèques

de l'UdeM. 


\section{Des idées reçues en santé mondiale}

\section{Valéry Ridde et Fatoumata Ouattara}

DOI : 10.4000/books.pum.3607

Éditeur : Presses de l'Université de Montréal

Lieu d'édition : Montréal

Année d'édition : 2015

Date de mise en ligne : 7 novembre 2017

Collection : PUM

EAN électronique : 9782821895461

\section{OpenEdition}

\section{Books}

https://books.openedition.org

\section{Édition imprimée}

EAN (Édition imprimée) : 9782760635234

Nombre de pages : 250

\section{Référence électronique}

RIDDE, Valéry; OUATTARA, Fatoumata. Des idées reçues en santé mondiale. Nouvelle édition [en ligne]. Montréal : Presses de l'Université de Montréal, 2015 (généré le 03 février 2022). Disponible sur Internet : <http://books.openedition.org/pum/3607>. ISBN : 9782821895461 . DOI : https://doi.org/ 10.4000/books.pum.3607.

(C) Presses de l'Université de Montréal, 2015 Conditions d'utilisation :

http://www.openedition.org/6540 


\section{RÉSUMÉS}

"J'ai connu la tentation du cliché ", avoue le philosophe Alain Badiou en entrevue. Nous voilà rassurés: même les plus grands risquent de tomber dans les poncifs ou, comme l'expliquait Platon, dans une vision cynique et pauvre du monde. Ce livre pose le problème de l'obscurantisme lié à l'absence d'accès aux connaissances.

Le lecteur est donc convié à une aventure de vérification. Des spécialistes internationaux de plusieurs domaines (santé publique, anthropologie, sociologie, histoire, économie) déconstruisent ici quelques idées reçues autour de thèmes très variés : sida, santé maternelle, reproductive et sexuelle, accès aux soins, offre de soins, environnement, nutrition... Le pari consiste à mettre en lumière l'importance d'une argumentation critique nuancée en examinant des idées largement véhiculées, c'est-à-dire celles qui ont cours dans le grand public. Par sa dimension éclectique, cet ouvrage est aussi divertissant qu'instructif pour lutter contre les clichés néfastes au progrès des Nations.

\section{VALÉRY RIDDE}

Professeur agrégé de santé publique à l'École de santé publique de l'Université de Montréal, titulaire d'une chaire de recherche en santé publique appliquée des Instituts de recherche en santé du Canada (IRSC) et chercheur à l'Institut de recherche en santé publique de l'Université de Montréal (IRSPUM)

\section{FATOUMATA OUATTARA}

Docteure en anthropologie sociale de l'École des hautes études en sciences (EHESS) de Marseille. Elle est chargée de recherche à l'Institut de recherche pour le développement (IRD) en France et ses travaux portent sur la santé de la reproduction et les catégories morales. 

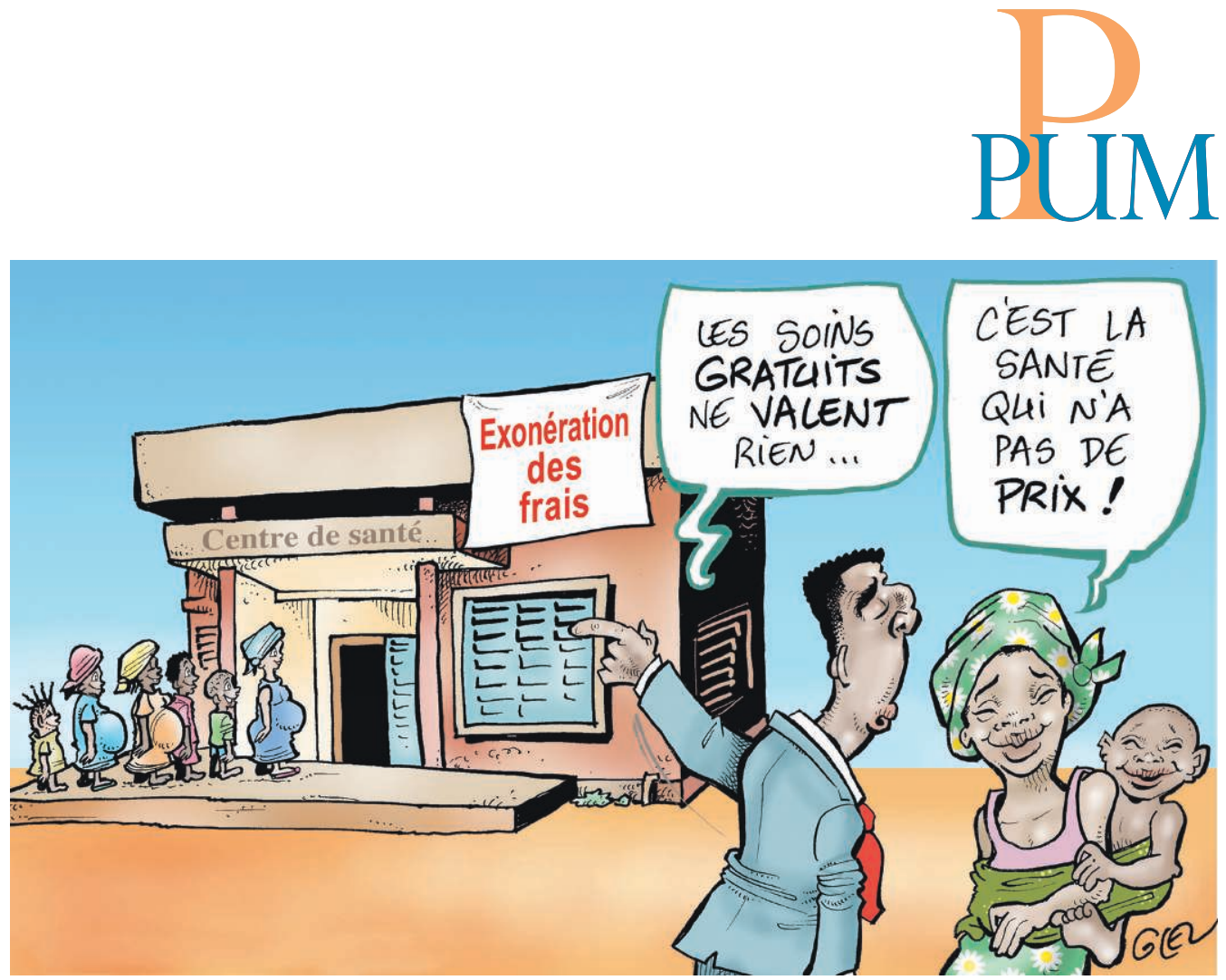

\section{Des idées reçues en santé mondiale}

Sous la direction de

Valéry Ridde et Fatoumata Ouattara 

DES IDÉES REÇUES EN SANTÉ MONDIALE 

Sous la direction de

Valéry Ridde et Fatoumata Ouattara

\section{DES IDÉES REÇUES EN SANTÉ MONDIALE}




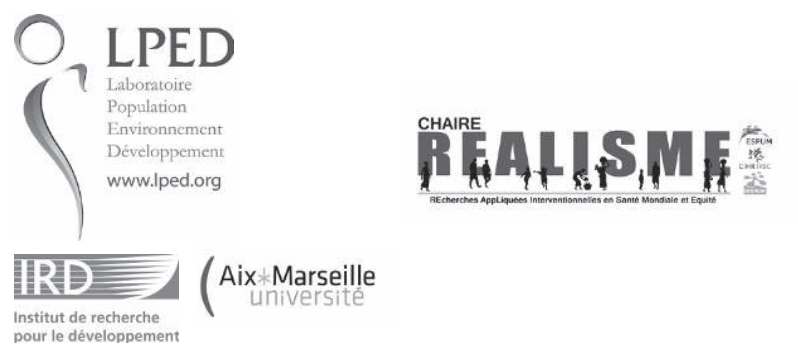

Le dessin de couverture est une réalisation de $\mathrm{D}$. Glez, d'après une idée de L. Queuille et V. Ridde, produit par HELP/CRCHUM/ECHO.

Catalogage avant publication de Bibliothèque et Archives nationales du Québec et Bibliothèque et Archives Canada

Vedette principale au titre:

Des idées reçues en santé mondiale

(PUM)

(Libre accès)

Comprend des références bibliographiques.

ISBN 978-2-7606-3523-4

1. Santé mondiale. I. Ridde, Valéry, 1969- . II. Ouattara, Fatoumata. III. Collection: PUM. RA441.133 2015

362.1

C2015-941583-7

Mise en pages: Folio infographie

ISBN (papier) : 978-2-7606-3523-4

ISBN (PDF) : 978-2-7606-3524-1

ISBN (ePub) : 978-2-7606-3525-8

Dépôt légal: $4^{\mathrm{e}}$ trimestre 2015

Bibliothèque et Archives nationales du Québec

(c) Les Presses de l'Université de Montréal, 2015

Les Presses de l'Université de Montréal remercient de leur soutien financier le Conseil des arts du Canada et la Société de développement des entreprises culturelles du Québec (SODEC).

\section{Canadä̀}

IMPRIMÉ AU CANADA 


\section{Préface}

L'ouvrage dirigé par Valéry Ridde et Fatoumata Ouattara prend place dans la vaste littérature sur les «idées reçues», dont ils citent dans leur introduction quelques exemples, parmi lesquels on retiendra avec eux, outre l'incontournable Gustave Flaubert dans la catégorie des illustres fondateurs, Georges Courade dans la catégorie des chercheurs contemporains en sciences sociales. Le livre dirigé par Courade, L’Afrique des idées reçues, offre en effet deux similitudes avec celui-ci: il concerne l'Afrique (qui est le site principal - mais non exclusif - de références pour les auteurs rassemblés par Ridde et Ouattara); et il est lui aussi écrit par un ensemble de chercheurs soucieux d'opposer des vérités scientifiques aux clichés habituels.

La spécificité du présent ouvrage réside bien évidemment en son thème, la santé mondiale, dans laquelle s'inscrivent les 46 «idées reçues » qui sont ici réfutées. La santé mondiale est de prime abord une affaire de spécialistes, aux confins de la santé publique, de l'épidémiologie, de la géographie de la santé ou de l'économie de la santé; certains diraient qu'il s'agit en fait plus simplement de la santé publique dans les pays du Sud. Mais bien évidemment, la santé mondiale concerne aussi les interactions sanitaires prenant place quotidiennement dans ces pays, et donc tant le personnel de santé et les malades que les responsables politiques. Tout le monde a donc, à divers égards, son mot à dire sur ces sujets. Et chacun peut donc être pris en flagrant délit de profération d'une «idée reçue».

C'est ainsi que les «idées reçues» qui sont présentées dans cet ouvrage reflètent des rapports très différents aux réalités sanitaires. Une partie relève clairement de clichés communs ou de stéréotypes profanes (comme «Pour se soigner, les sociétés africaines sont solidaires»). Mais d'autres expriment des points de vue répandus au sein des professions médicales 
(comme «Les médicaments du marché informel sont des faux médicaments»), et certaines peuvent même être activement soutenues par des spécialistes reconnus («Financer le système de santé sur la base des résultats sauve des vies»).

On peut dès lors s'interroger sur ce qu'est une «idée reçue» en santé mondiale et sur le statut de la réfutation dont elle fait l'objet. S'agit-il d'opposer la vérité de la science aux erreurs du sens commun, dans une version médicalisée de la «rupture épistémologique» prônée il y a longtemps par Bourdieu, Chamboredon et Passeron dans Le métier de sociologue (comme avec «Les méthodes contraceptives rendent stérile»)? Ou bien s'agit-il, dans l'espace propre des débats scientifiques, de démontrer autant que faire se peut le caractère erroné des assertions de certains collègues (comme avec «Les comités d'éthique garantissent le caractère éthique des recherches en santé») ? S’agit-il de se mettre sur le terrain des politiques de santé pour dénoncer les naïvetés ou les postulats fallacieux qui s'y révèlent (comme avec «La présentation des preuves scientifiques conduit les décideurs à prendre des décisions rationnelles»)? On bien s'agit-il tout simplement, sur un mode plus prosaïque, de plaider pour la complexité face à des simplifications excessives (comme avec «C'est à cause de leur culture que certains patients ne suivent pas leur traitement»)?

Il s'agit en fait de tout cela à la fois dans cet ouvrage, dont le mérite est justement d'ouvrir de façon argumentée et pédagogique de multiples débats, qui se situent dans des registres argumentatifs variés, plutôt que d'asséner des vérités ou de défendre des dogmes. Le procédé rhétorique de la déconstruction d' «idées reçues» n'est pas ici utilisé pour dénoncer ou ridiculiser ceux qui les proféreraient, mais pour inviter à aller au-delà de certaines apparences et à mieux poser des problèmes importants. Ce qui semble «aller de soi », pour des malades, pour des professionnels de santé, pour des décideurs, et même pour des chercheurs, «ne va pas de soi », et il faut y regarder de plus près : tel est, à mes yeux, le message central de toutes les contributions, en leur variété. Au fil des discours, des débats ou des textes, chacun de nous a déjà recouru, recourt, et recourra encore, çà et là, à des interprétations stéréotypées ou à des généralisations paresseuses : ce livre nous incite à une saine vigilance, à plus de rigueur, et c'est là tout son intérêt. Sur nombre des sujets évoqués, et au-delà des clichés évidents qui sont réfutés au fil des articles, la discussion n'est pas close et doit se poursuivre. Mais elle gagnera à tenir mieux compte désormais des 
données empiriques disponibles et de l'analyse critique de celles-ci: c'est ainsi que je comprends le but de cet ouvrage, et c'est pour cela que j'en conseille la lecture.

Mais les «idées reçues» ont aussi une dimension «idéologique». Dans le monde du développement (et la santé mondiale est un secteur du développement comme un autre) prévalent trop de modèles standardisés (la langue anglaise les caractérise avec acuité: «magic bullet» ou «one size fits all»), souvent fondés sur des «idées reçues» ou utilisant à tout le moins des «idées reçues» à titre de légitimation. Les idéologies politiques et/ou scientifiques sont le plus souvent au cœur de ces processus de production de mécanismes miraculeux, dits «à haut facteur d'impact», fréquemment issus du «new public management», et censés être efficients dans des contextes très variés. La diffusion massive, par la Banque mondiale, du paiement basé sur la performance dans les systèmes de santé en Afrique en est une illustration d'actualité. C’est une caractéristique commune des idéologies et des «idées reçues» que d'ignorer la complexité irréductible des contextes.

Certes, il serait illusoire de croire que l'univers scientifique pourrait être débarrassé des idéologies qui prolifèrent dans son environnement intellectuel et politique. On ne peut opposer une sphère scientifique pure, fondée sur la preuve, et une sphère sociale, où règneraient les "idées reçues». Les pressions idéologiques s'exercent en permanence au sein même de l'univers scientifique, et aucune procédure, aucun protocole, aucun dispositif d'enquête, ne peuvent nous en débarrasser une fois pour toutes. Mais on ne peut pour autant se résigner à être la proie des préjugés, des stéréotypes, des pré-conceptions, des clichés ou des dogmes. Dans cette lutte constante, complexe, dialectique, entre les productions de savoirs (nécessaires pour comprendre le monde et régulées par la quête de véracité ou à tout le moins de plausibilité) et les idéologies (nécessaires pour le transformer, et régulées par la quête d'efficacité), il n'est pas d'autre voie pour un chercheur que l'exercice de la pensée critique, la promotion de débats basés sur des arguments empiriquement fondés, le recours aux contre-exemples, la réfutation des idées reçues.

Jean-Pierre Olivier de Sardan

Directeur de recherche émérite au CNRS France Directeur d'études à l'EHESS France Chercheur au LASDEL Niger 

«La fonction du mythe, c'est d'évacuer le réel.»

Roland BARTHES, 1970

"Le mythe, à trop s'enfler, doit éclater.»

Edgar MORIN, 1969 



\title{
Introduction
}

\author{
Valéry Ridde et Fatoumata Ouattara
}

Préjugés, stéréotypes, clichés, mythes, idées reçues, voici autant de termes apparaissant à la fois comme des allégories du langage commun et comme des spectres guettant toute réflexion intellectuelle. Pour peu que celle-ci relève d'une procédure d'analyse scientifique de la réalité, d'une étude rigoureuse des situations, le risque du cliché n'est jamais loin.

"J'ai connu la tentation du cliché», dit Alain Badiou dans une entrevue télévisée'. Nous voilà rassurés: même les plus grands philosophes contemporains risquent de tomber dans ces poncifs ou, pour reprendre ses mots évoquant la définition des clichés par Platon, dans cette vision cynique et pauvre du monde. Badiou nous rappelle aussi que Platon définissait la philosophie comme la lutte contre les clichés, c'est-à-dire contre les opinions (« une parole choisie par l'histoire», dit Barthes) non fondées, ou ces «fausses évidences» que sont les mythes, selon Barthes (1970). On se rappellera comment Raymond Boudon, en faisant un lien entre l'idéologie et les idées reçues, appréhendait les manières par lesquelles on adhère aux idées fausses (1986). Dans un autre contexte, et plus récemment, l'ensemble de ces mots et expressions renvoie «à des notions de répétitions et d'informations figées» (Observatoire Régional de la Santé NordPas-de-Calais, 2013: 1). Il ne s'agira pas dans ce livre de trouver des définitions formelles ou ce que signifierait une idée reçue, par exemple. Il ne s'agira pas non plus de déterminer les conditions d'émergence, de circulation ou de temporalité de telles idées. D’ailleurs, une des idées reçues en la matière est que «les idées reçues ont la vie dure»!

1. https://www.youtube.com/watch?v=AOSTZSnoPrE 
Ainsi, loin de nous l'idée d'inviter le lecteur à une réflexion de nature philosophique sur le bien-fondé des opinions ou encore le caractère objectif de la science. Mais il faut bien reconnaître, comme Bernard Rentier ${ }^{2}$, recteur de l'Université de Liège (Belgique), le rappelait récemment dans son plaidoyer en faveur d'un accès libre aux publications scientifiques, que l'obscurantisme est souvent lié à l'absence d'accès aux connaissances. Barthes (1970) évoquait justement ce «mythe obscurantiste» (39) et le «divorce accablant de la connaissance et de la mythologie ${ }^{3} »(72)$.

Qui ne se souvient pas de cette fameuse rumeur d'Orléans, en France, à propos de la disparition de femmes, analysée par une équipe dirigée par Edgar Morin à la fin des années 1960 (Morin, 1969)? L'équipe de sociologues s'était engagée dans une «entreprise de démythification» (9), montrant notamment «la très remarquable absence de souci de vérifier " (113). C'est dans cette aventure de vérification que nous souhaitons emmener le lecteur intéressé par les faits de la santé mondiale, en mettant en évidence la place cruciale de l'argumentation critique qui s'accompagne du souci de la nuance.

Les origines de cet ouvrage collectif sont multiples. D’abord, nos recherches sont essentiellement réalisées en Afrique, dans le domaine de la santé. L'Afrique n’a pas la préséance sur les autres continents pour ce qui est de la circulation d'idées reçues, de mythes et autres rumeurs; il suffit de penser au concept d'ethnie, qui représente bien cette mondialisation des croyances (Amselle et M’Bokolo, 1999). Dans un ouvrage sur L'Afrique des idées reçues (Courade, 2006), on discute par exemple du pouvoir des femmes ou des vertus communautaires des sociétés africaines dans un contexte où le traditionalisme culturel de ces sociétés est souvent convoqué abusivement (Olivier de Sardan, 1999, 2010). Ce travail fort passionnant nous a convaincu de l'intérêt de poursuivre notre réflexion, en faveur d'une diffusion plus grande des plus récentes données sur certains sujets de santé mondiale.

Ensuite, nous nous sommes rapidement rendu compte que notre désir de partage des connaissances avec le plus grand nombre n'était pas solitaire. Bien d'autres avant nous s'étaient engagés dans des publications visant à contrer les idées reçues sur l'histoire ou sur l'Asie et l'Orient

2. http://tv.uqam.ca/Default.aspx $? \mathrm{v}=53835 \& \mathrm{p}=\mathrm{O}$

3. Citation extraite d'un des rares mythes concernant l'Afrique dans son célèbre ouvrage. 
(Historia, 2010; Poulet, 2009). Tous les ans, le grand ouvrage des idées reçues est publié à destination du grand public (Éditions Cavalier Bleu) et récemment, au Québec, a été publié un livre où 50 idées reçues sont déconstruites par des chercheurs (Lamy, 2012).

Enfin, par notre engagement dans le monde de la recherche appliquée et de la pratique de la santé publique en Afrique, au plus près des acteurs concernés depuis de nombreuses années, nous avons affronté au quotidien l'expression d'idées reçues. L'article «Pourquoi ces mères indifférentes? Ou comment faire la part du culturel» publié par Marie-Cécile et Edmond Ortigues (1993) souligne l'intérêt à dépasser les discours à forts traits culturalistes de soignants à l'égard des usagers. Plus récemment, et en rapport direct avec nos domaines de recherche, on se souviendra par exemple de ce professeur de gynécologie osant affirmer haut et fort devant ses pairs lors d'une conférence nationale dans un pays d'Afrique de l'Ouest que rendre les accouchements gratuits allait provoquer plus de naissances. Évidemment, les preuves scientifiques vont à l'encontre de cette croyance (Ridde, Queuille et Ndour, 2014), mais cet exemple montre que même les esprits soi-disant les mieux formés (la fameuse médecine fondée sur des preuves) ne sont pas à l'abri, comme osait le dire Badiou, de dérives discursives, $\mathrm{du}$ «recours à une fausse nature [...] d'images pauvres, incomplètes» (Barthes, 1970: 222-223).

En tant que chercheurs travaillant sur des faits de santé, cela nous rappelle les débats sur le culturalisme pratique de la santé publique, notamment en France (Fassin, 2001). N'est-ce pas dans ce sens que Didier Fassin et Anne-Claire Defossez, à partir d'une expérience d'enquête de terrain en Équateur, allaient jusqu'à qualifier le dialogue entre sciences sociales et santé publique de «liaison dangereuse» (Fassin et Defossez, 1992)? Dès lors que ces relations sont envisagées de façon critique, il devient alors nécessaire de considérer, par exemple, l'indicateur de mortalité maternelle dans sa globalité et non sous le seul angle sanitaire que lui assignent des institutions internationales. «L'opinion que se forgent les médecins et les décideurs au sujet des populations qu'ils soignent ou administrent, de leurs comportements et des raisons ou des logiques qui les sous-tendent, se fondent souvent sur une connaissance qui ressortit à la sociologie ou à l'anthropologie spontanées, c'est-à-dire à une sorte de bon sens. Il en résulte une série d'idées reçues dont la plupart résistent mal à l'épreuve des faits.» (Ibid.: 28). Plus de vingt ans après la publication 
de cet article, ce rappel n'a pas pris une ride. Ainsi, en santé mondiale (enjeux de santé dépassant les territoires nationaux et essentiellement concernés par des questions d'équité), nous ne sommes pas à l'abri de l'existence, ou de la persistance, d'idées reçues parfois davantage chargées de préjugés que de preuves scientifiques. Certains vont affirmer par exemple que les hommes peuls préfèrent dépenser de l'argent pour soigner leurs animaux que leurs enfants; que les femmes africaines n'aiment pas être accouchées par des hommes; ou que les patients doivent payer les soins pour les valoriser.

Ainsi, cet ouvrage collectif vise à présenter et à déconstruire quelques idées reçues en santé mondiale selon une perspective interdisciplinaire et sur des bases scientifiques à travers des exemples touchant l'ensemble des continents. La notion d'idée est abordée au sens large du terme, soit comme recouvrant les croyances, les valeurs, les préférences ou les principes véhiculés par les acteurs sociaux au regard de sujets touchant la santé (Béland et Cox, 2011). Certains textes traitent d'idées arrêtées, attestant ainsi la particularité de populations: des sociétés africaines qui seraient plus solidaires en situation de maladie, des femmes africaines qui feraient plus d'enfants, l'homosexualité qui n'existerait pas en Afrique. Parfois, les textes développés renvoient à la déconstruction d'idées circulant de façon plus implicite, par exemple, l'idée selon laquelle les méthodes contraceptives rendent stérile ou bien celle sur le fait que les prestations confessionnelles servent les pauvres. Enfin, d'autres textes s'attachent à commenter et à démonter des idées fortement associées aux investigations scientifiques, comme celui sur les questions que le VIH/sida pose aux politiques de santé ou alors celui sur la mise en place d'un système de financement basé sur les résultats.

Pour déconstruire certaines idées reçues, nous avons réuni des spécialistes internationaux de multiples domaines d'expertise (santé publique, anthropologie, sociologie, histoire, économie) concernant des contextes géographiques divers (Asie, Afrique, Amérique latine, etc.) et autour des thèmes les plus variés possible (VIH, santé maternelle, santé de la reproduction, santé sexuelle, accès aux soins, offre de soins, nutrition, environnement). Si le thème de la santé mondiale est l'axe fédérateur de l'ensemble des textes, ceux-ci sont regroupés autour de six thématiques différentes: les politiques et les systèmes de santé; la prévention et les soins; la santé maternelle et infantile; les populations vulnérables et l'équité; les singularités des populations; la recherche en santé. 
Notons que nous nous intéressons aux idées largement véhiculées, soit celles qui ont cours dans le grand public, et non pas aux idées d'experts ou aux débats entre scientifiques. Nous avons expressément demandé aux auteurs de ne pas user d'un jargon scientifique difficilement accessible en dehors du «jeu des perles de verre», comme dirait Hermann Hesse (1999), mais de s'adresser à un large public. Cela n'a pas toujours été facile, d'autant plus qu'il y avait la contrainte de rédiger des textes courts. Mais les auteurs ont tout de même pu partager leur plus intime connaissance de leur sujet d'expertise pour déconstruire ces idées reçues en santé mondiale. Aussi, au lieu d'une longue liste de références scientifiques qui ne donne que rarement envie de vérifier, chaque chapitre mentionne trois références clés, récentes et, pour certaines, accessibles sur Internet, que les lecteurs passionnés pourront lire pour approfondir le sujet.

Au XIX ${ }^{e}$ siècle, Gustave Flaubert s'est amusé à publier un dictionnaire des idées reçues, qui était surtout de nature humoristique. Notre ouvrage sur les idées reçues en santé mondiale n'est malheureusement pas humoristique, mais nous espérons que sa lecture sera aussi divertissante qu'instructive par sa dimension éclectique pour contribuer à lutter contre ces clichés néfastes au progrès des nations.

\section{Références}

Amselle, J.-L. et M'Boкоlo, E. (1999). Au cour de l'ethnie: ethnies, tribalisme et État en Afrique. Paris: La Découverte.

Barthes, R. (1970). Mythologies. Paris: Éditions du Seuil.

BÉland, D. et Cox, R. H. (dir.). (2011). Ideas and Politics in Social Science Research. New York: Oxford University Press.

Boudon, R. (1986). L'idéologie, ou, L'origine des idées reçues. Paris: Fayard.

Courade, G. (dir.). (2006). L’Afrique des idées reçues. Paris: Belin.

FAssin, D. (2001). Le culturalisme pratique de la santé publique. Critique d'un sens commun. In J.-P. Dozon et D. FAssin (dir.), Critique de la santé publique. Une approche anthropologique (p. 181-208). Paris: Balland.

Fassin, D. et Defossez, A.-C. (1992). Une liaison dangereuse. Sciences sociales et santé publique dans les programmes de réduction de la mortalité maternelle en Équateur. Cahiers des Sciences Humaines, 28 (1): 23-36.

Hesse, H. (1999). Le jeu des perles de verre. Essai de biographie du Magister Ludi Joseph Valet: accompagné de ses écrits posthumes. Paris: Librairie générale française.

Historia. (2010). 150 idées reçues sur l'histoire. Paris: First. 
Lamy, G. (dir.). (2012). C’est faux! 50 idées déconstruites par des spécialistes. Québec (Québec): Septentrion.

Morin, E. (1969). La rumeur d'Orléans. Paris: Éditions du Seuil.

Observatoire Régional de la Santé Nord-Pas-de-Calais. (2013). Observations inattendues... et capricieuses de la santé. Clichés. http://www.orsnpdc.org/ observation/287339_1cliches.pdf

Olivier de SARdAn, J.-P. (2010). Le culturalisme traditionaliste africaniste. Analyse d'une idéologie scientifique. Cahiers d'études africaines, L (2-3-4)(198-199-200), : 419-453.

Olivier de Sardan, J.-P. (1999). Sociétés et développement. In D. Fassin et Y. Jaffré, Sociétés, développement et santé, Ellipses/AUPELF, 28-37.

Ortigues M.-C. et E. (1993). Pourquoi Ces mères indifférentes? ou COMMent faire la part du Culturel. Psychopathologie Africaine, XXV (1): 5-31.

Palier, B. et Surel, Y. (2005). Les «trois I» et l'analyse de l'État en action. Revue française de science politique, 55(1): 7-32.

Poulet, R. (2009). Dictionnaire des idées reçues sur l'Asie et l'Orient. Les Editions du Zaporogue.

Ridde, V., Queuille, L. et Ndour, M. (2014). Nine misconceptions about free healthcare in sub-Saharan Africa. Development Studies Research, 1(1): 54-63. doi :10.1080/21665095.2014.925785 


\section{LES POLITIQUES ET LES SYSTèMES DE SANTÉ}





\section{L'implantation de la médecine occidentale devrait entraîner la disparition des médecines locales dans les pays à faible revenu}

Nicolas Vornax

Le sujet des médecines locales des pays à faible revenu est depuis longtemps discuté dans le champ de la santé publique internationale. Les anthropologues ont d'ailleurs analysé ces médecines, pour identifier des praticiens locaux, comprendre les significations sociales de la maladie et les pratiques qui en découlaient. Ainsi, on a bien compris que les mondes dans lesquels on intervenait au nom du progrès et du développement dans le domaine de la santé n'étaient pas vides de contenu. Le domaine de la santé n'était pas sans systèmes médicaux ou sans traditions médicales. Les programmes de santé publique pouvaient se heurter à ces pratiques enracinées de longue date dans des sociétés et des cultures. À ce titre, on a pu, et l'on peut encore penser que ces héritages du passé devraient s'effacer lorsque la médecine scientifique occidentale sera suffisamment intégrée, lorsque les paysages seront peuplés de structures de santé biomédicales, lorsque ces dernières seront bien organisées et riches en matériel divers, et lorsque l'on aura formé suffisamment de médecins et d'infirmières pour les habiter. Ce doit être pour cela qu'on qualifie de manière abusive ces médecines de "traditionnelles», en les inscrivant ainsi dans un ordre de progression culturelle où le moderne doit inévitablement prendre la place du traditionnel. 
Bref, l'occidentalisation des systèmes médicaux à partir d'une diffusion de modèles et de motifs en provenance de pays riches devrait, semble$\mathrm{t}$-il, transformer le rapport que les personnes des pays à bas et moyen revenu entretiennent avec les médecines locales. Ces personnes devraient alors s'orienter différemment dans leurs recours aux soins. Elles devraient privilégier la médecine occidentale en raison de sa grande efficacité, de sa provenance, et tourner progressivement le dos à des pratiques considérées comme peu efficaces, et aux praticiens locaux rangés habituellement dans les catégories de tradipraticiens, de guérisseurs, de sorcierschamans, d'herboristes, voire de charlatans.

Évidemment, l'ordre des choses n'est pas si simple, et l'on constate qu'il ne suffit pas de la présence des uns pour produire la disparition des autres. Le marché des offres de soins n'est pas une garde-robe où le neuf remplace le vieux linge démodé. La vitalité des médecines locales est toujours aussi forte. Elle l'est par exemple dans des pays d'Asie qui la proposent d'une manière officielle, comme un service conjoint aux services biomédicaux, et se présente à travers de multiples initiatives de valorisation et de reconnaissance de ces médecines dans des politiques nationales de santé développées ici et là.

On comprend donc que les médecines et traditions médicales locales non scientifiques sont encore en vigueur dans les pays à faible et moyen revenu. Elles semblent tirer leur épingle du jeu sur un marché des offres de soins toujours plus grand et très compétitif. La situation en Occident des médecines et des thérapies dites alternatives montre même que l'hyper-modernité et les avancées technologiques ne changent rien. Effectivement, en dépit du développement important de la biomédecine en Occident depuis deux siècles, en dépit de nouvelles technologies médicales diagnostiques et thérapeutiques très performantes et d'un accès à des services de santé en augmentation, les médecines alternatives et complémentaires sont toujours très présentes dans les itinéraires thérapeutiques des personnes malades. En témoigne une étude réalisée en 2006 sur l'usage de ces espaces de soins chez 2000 Canadiens. Il apparait dans ces travaux qu'environ $70 \%$ des Canadiens et des Québécois ont eu recours à ces pratiques dans leur vie et qu'environ la moitié avait consulté un thérapeute de ce secteur durant l'année précédant l'enquête. Il suffit encore de naviguer sur le site Internet de Passeport Santé (http://www.passeportsante.net/fr) pour constater que l'abécédaire de ces thérapies est impres- 
sionnant et que de nouvelles formes thérapeutiques sont apparues durant les dernières années.

\section{Des médecines utiles et indéracinables}

Il semble donc difficile de soutenir un lien de cause à effet direct entre la présence de la biomédecine et la disparition potentielle des thérapeutes et des thérapies de tout acabit. Il faut dire par ailleurs que plusieurs facteurs jouent en faveur de la présence et de la popularité de ces dernières. Ces facteurs renvoient à une accessibilité géographique et économique à des thérapeutes en tout temps. Retenons encore que l'accessibilité renvoie aussi à la manière dont on comprend, explique et interprète les problèmes dans ces médecines, comme aux procédures et aux logiques par lesquelles on passe pour les résoudre. Par exemple, le recours à ces médecines est parfois pertinent parce que le sens que le thérapeute donne au mal est bien compris par ceux qui le consultent, parce que son explication s'enracine dans le monde des personnes en souffrance. C'est le cas notamment en Haïti, où l'on continue de recourir à la médecine vaudou parce que les consultants y entendent que leurs infortunes relèvent du manque de soins à l'endroit de relations avec leurs ancêtres, avec des entités non humaines (les lwa) que l'on honore depuis plusieurs générations dans les habitations familiales, à qui l'on voue parfois un culte d'adoration individuelle. Dans leurs théories sur la maladie, les thérapeutes évoquent encore les relations sociales dans lesquelles se présentent l'envie, la convoitise, la jalousie, les conflits ou des comportements non tolérés. Ces «ingrédients » seraient à l'origine de nombreux problèmes en Haïti. Le malheur suppose donc la nécessité de revenir sur la place que le malade et les siens occupent dans une société et un monde haïtiens complexes, meublés de divers éléments avec lesquels les consultants sont très familiers. Ceux-là accueillent ainsi favorablement ce type de lecture et les procédures thérapeutiques qui s'ensuivent, et ce, en raison d'une proximité culturelle. Les Haïtiens qui consultent dans le vaudou s'y reconnaissent et reconnaissent les lieux dans lesquels ils ont appris à exister, et dans lesquels les problèmes émergent.

Une correspondance entre les discours des thérapeutes et des soignés, une inscription du problème dans des réseaux sémantiques communs, la mobilisation de modèles explicatifs du malheur connus de part et d'autre, des renvois à des normes sociales, des ordres de moralité, des modes de 
vie, des agents morbides divers, ou d'autres référents propres à une société ou à un groupe, rapprochent ainsi les médecines locales de ceux qui les utilisent. Le familier, l'intelligible et la reconnaissance de ce que l'on est, de la manière dont on se définit et dont on vit, participent de cette accessibilité. La quête de bien-être et de guérison se mêle par conséquent à une quête et une offre de sens qui ont aussi leur importance dans les logiques et les motivations du recours aux soins.

Il faut donc savoir que le recours à des médecines ne correspond pas uniquement à des quêtes de guérison, mais qu'il comporte une dimension sociale qui compte pour beaucoup. La permanence de ces médecines coïncide avec la permanence (et parfois l'affirmation et la revendication) de systèmes et d'ordres sociaux, de référents identitaires qui disent beaucoup de ce que nous sommes, de ce que nous voulons être et de ce que nous devenons.

En conclusion, penser la présence des médecines locales et leur disparition dépasse les questions d'efficacité, de preuves scientifiques ou de méthodes thérapeutiques qui viennent systématiquement à l'esprit de ceux qui ont tendance à entretenir l'idée reçue d'une disparition des médecines locales au profit de la médecine moderne occidentale. Les médecines et thérapies qui côtoient la médecine scientifique qu'on essaie d'implanter ici et là devraient donc subsister, à moins qu'on engage une lutte féroce contre elles. Il en va d'une certaine permanence et d'une continuité de sens. Il en va d'un rôle social et pas uniquement thérapeutique et, bien souvent, d'une autre manière de comprendre le monde et de s'y inscrire pour être bien et se protéger des infortunes.

L'Organisation mondiale de la santé (OMS) prescrit d'ailleurs une attitude positive à l'endroit des médecines du monde en suggérant des politiques d'intégration des médecines, une collaboration, ou tout au moins une tolérance de ces pratiques et praticiens. Les paysages médicaux qui devraient se présenter dans le futur nous apparaissent relever beaucoup plus du pluralisme, de la juxtaposition de pratiques, de la combinaison et de l'emprunt, que de la disparition de traditions médicales et de l'imposition d'une médecine scientifique appuyée par des programmes de santé internationale. Prenons le temps d'examiner les lieux de ces interventions et nous le constaterons très vite. De là, nous pourrons en partie changer nos idées reçues héritées d'un passé néocolonial et de théories évolutionnistes bien dépassées! Évidemment, cet état de faits ne doit pas 
non plus étouffer les projets d'intégration de la médecine scientifique là où on en est dépourvu. Mais le faire n'oblige pas d'imposer des modèles uniques et d'être animé des attitudes paternalistes qui imprègnent parfois le champ de la santé internationale.

\section{Pour aller plus loin}

Esmail, N. (2007). Complementary and alternative medicine in Canada: trends in use and public attitudes, 1997-2006. Public Policy Sources, 87.

Organisation MONDIALE DE LA SANTÉ (2002). Stratégie de l'OMS pour la médecine traditionnelle. Genève, Organisation mondiale de la santé.

VONARx, N. (2012). Le vodou haïtien: entre médecine, magie et religion. Presses universitaires de Rennes. 


\title{
Les politiques de santé ne se fondent que sur des critères rationnels
}

\author{
Sanni Yaya et Mamadou Barry
}

Le recours au savoir comme moyen d'aide à l'élaboration, à la hiérarchisation et à la mise en œuvre des politiques n'est pas un phénomène contemporain. Aujourd'hui encore, la recherche d'une plus grande efficacité dans les politiques publiques, notamment en matière de santé, veut inciter l'action publique à davantage de rationalité et d'objectivité. Dans cette quête, une idée est constamment mise en avant: utiliser les données sur la santé pour concevoir, mettre en œuvre et évaluer les politiques de santé. L'Organisation mondiale de la santé (OMS) souligne que l'atteinte des objectifs sanitaires à l'échelle internationale exige une importante mobilisation de données scientifiques rigoureuses. Elle insiste ainsi sur la nécessité d'utiliser les données sanitaires afin de surveiller l'état de santé des populations, de comprendre les espérances de vie faibles, d'évaluer l'émergence des maladies non transmissibles, de contrôler la propagation des maladies transmissibles et d'élaborer des politiques de santé conséquentes.

Depuis une décennie, bailleurs et bénéficiaires de l'aide publique au développement se sont promis de donner la priorité aux problèmes de santé en utilisant des données probantes compte tenu des interventions efficaces disponibles. Ces différents appels à l'utilisation de preuves scien- 
tifiques laissent penser que les politiques de santé seraient exclusivement déterminées par des considérations objectives sur la santé.

Mais la réalité est tout autre. La définition même d'une question en tant que politique de santé est, par essence, de nature politique. Cela concerne la conception de la santé que ses promoteurs souhaitent mettre en avant, à la lumière des principes, valeurs et intérêts qui sont les leurs et au regard du cheminement proposé pour élaborer et rendre cohérente cette politique de santé. Les décisions portant sur les politiques de santé sont très souvent d'une grande complexité. Contrairement au modèle du choix rationnel qui suppose une parfaite connaissance des différentes options, de leurs conséquences, de leur utilité relativement aux buts poursuivis, les choix en santé illustrent plus souvent quautrement les déviations du raisonnement réel par rapport à la rationalité véhiculée dans les discours officiels.

\section{La vision dominante}

Au sein des organisations internationales intervenant dans le domaine de la santé, un nouveau consensus se fait jour autour du concept de données objectives en matière d'élaboration des politiques de santé. Ce modèle de décision fondé sur une rationalité néoclassique est employé dans de nombreuses analyses sur les politiques socioéconomiques. Il se fonde sur l'utilisation d'analyses coûts-bénéfices en tant que moyen d'aide à la hiérarchisation et à la décision. Une telle approche repose sur l'hypothèse d'une capacité des acteurs à définir avec soin la nature des problèmes auxquels ils font face et à proposer des solutions différentes. L'évaluation de ces solutions sur la base d'un ensemble de critères objectifs devrait fonder la décision.

Mais cette approche repose sur des présupposés très contestables. En effet, bien souvent, les concepteurs de politiques, souvent appuyés d'experts d'une question, se mobilisent pour proposer du problème qui les intéresse une définition différente de celle qui est adoptée par les décideurs, afin de les amener à modifier leurs perceptions dans un sens plus favorable aux intérêts des acteurs mobilisés. Le processus d'élaboration des politiques fonctionne comme un système de traitement de l'information. Ceci explique en partie pourquoi, et comment, les gouvernements accordent la priorité à tel problème plutôt qu'à tel autre, sans toujours considérer, comme il se doit, les données probantes. 


\section{Les financements internationaux}

En examinant les flux d'aide publique à la santé, on constate que les financements internationaux ne reposent pas sur des données probantes. En effet, certains enjeux de santé drainent l'attention des dirigeants politiques et des bailleurs de fonds, tandis que d'autres restent à l'arrière-plan, sans que ces différences ne s'expliquent par des facteurs objectifs tels que la morbidité et la mortalité ou par l'existence de moyens d'action efficients (c'est-à-dire rentables en termes de coût/efficacité).

TABLEAU 1

Charge de la maladie et financement de la santé dans les pays à revenu faible et intermédiaire

\begin{tabular}{l|c|c|c|c}
\hline \multicolumn{1}{c|}{ Catégorie } & $\begin{array}{c}\text { EVCI* } \\
\text { perdus en } \\
\text { milliers }\end{array}$ & $\begin{array}{c}\text { \% dans la } \\
\text { charge totale } \\
\text { de la maladie }\end{array}$ & $\begin{array}{c}\text { \% de l'aide } \\
\text { à la santé - } \\
\text { Interventions } \\
\text { directement } \\
\text { attribuables à } \\
\text { la maladie }\end{array}$ & $\begin{array}{c}\text { \% de l'aide } \\
\text { à la santé - } \\
\text { Interventions } \\
\text { directement et } \\
\text { indirectement } \\
\text { attribuables } \\
\text { à la maladie }\end{array}$ \\
\hline $\begin{array}{l}\text { Maladies infec- } \\
\text { tieuses sans MST } \\
\text { et VIH/sida }\end{array}$ & 327407 & 23,6 & 10,2 & 20,8 \\
\hline MST et VIH/sida & 80173 & 5,8 & 25,9 & 28,5 \\
\hline $\begin{array}{l}\text { Santé maternelle et } \\
\text { condition périnatale }\end{array}$ & 115494 & 8,3 & 1,0 & 19,8 \\
\hline $\begin{array}{l}\text { Déficiences } \\
\text { nutritionnelles }\end{array}$ & 29564 & 2,8 & 2,8 & 3,8 \\
\hline $\begin{array}{l}\text { Maladies non } \\
\text { transmissibles }\end{array}$ & 8842 & 48,9 & 0,0 & 22,1 \\
\hline Blessures & 55945 & 11,2 & 0,0 & 5,1 \\
\hline Total & $\mathbf{3 8 7 4 2 6}$ & $\mathbf{1 0 0 , 0}$ & $\mathbf{5 4 , 9}$ & $\mathbf{1 0 0 , 0}$ \\
\hline
\end{tabular}

* EVCl: espérance de vie corrigée en fonction de l'incapacité

Source: Présentation des auteurs d'après Mackellar (2005).

Pour mesurer les besoins de santé, l'espérance de vie corrigée en fonction de l'incapacité (EVCI) est un outil qui fait aujourd'hui autorité. Il permet de mesurer la charge de morbidité (ou invalidité) des différentes maladies et aide ainsi à une hiérarchisation rationnelle des dépenses. Ce tableau montre, par exemple, que la part des dépenses pour les programmes portant sur le VIH/sida (environ $30 \%$ de l'aide) est beaucoup 
plus élevée que le poids du VIH/sida dans la charge totale des maladies (environ $6 \%$ ) au début des années 200o. Inversement, des maladies transmissibles comme la pneumonie ou la diarrhée, qui tuent chaque année des millions de personnes et contre lesquelles on dispose de moyens d'intervention efficients, n'attirent que des financements modestes. Les maladies non transmissibles n'attirent aucun financement spécifique. Il apparaît donc que ce n'est pas l'importance d'un problème de santé qui prévaut dans les décisions politiques sur le financement de la santé. Et cela ne s'explique pas par un manque de données.

\section{Éléments d'explication}

Les acteurs qui composent la communauté des donateurs sont essentiellement des États, des organisations intergouvernementales, des ONG, des entreprises (industries pharmaceutiques, notamment) et des leaders d'opinion. Ce sont eux qui prennent les décisions relatives à l'organisation et à la mise en œuvre des politiques d'aide à la santé. Ces acteurs, qui peuvent partager des objectifs communs de développement, diffèrent d'abord par leurs statuts (publics et privés), ensuite par leurs intérêts et enfin par leurs valeurs et orientations. Les valeurs et orientations d'un acteur sont consubstantielles à sa perception d'un problème, de sa causalité, ainsi que des options disponibles pour le résoudre. Ainsi, on peut citer plusieurs exemples mettant en évidence cette image politique des bailleurs ou promoteurs de projets: la santé comme un droit, la santé comme un facteur de développement, la santé comme un facteur de sécurité et la santé comme un bien public. Les données sur la santé sont analysées et interprétées à l'aune de ces représentations sociopolitiques qui varient selon les époques, les espaces et les acteurs. Nous tenons là la raison principale des disparités entre maladies: ce que nous appelons réalité n'est pas quelque chose qui serait là, devant et hors de nous, indépendamment de l'observation humaine. La réalité n'est pas une donnée objective mais un construit social. Nous la construisons à travers des catégories mentales et des interactions sociales.

Un problème de santé se voit accorder de l'attention en raison moins de son importance objectivable que de la manière dont il est mis en évidence par ceux qui sont convaincus de son importance ou qui y ont intérêt. Cela ne signifie pas qu'il n'existe aucun lien entre la matérialité 
des faits et l'attention accordée à un problème, mais ce lien est distendu. Il est toujours influencé par des représentations sociales. Entre les faits ou les chiffres et les politiques mises en place s'intercale une intermédiation sociopolitique que l'on ne peut ignorer, voire éliminer.

Ainsi, la dynamique du choix en matière de politiques publiques repose moins sur un processus rationnel et davantage sur une approche socioconstructiviste. Les acteurs souhaitant se mobiliser autour de la santé, et ce, quel que soit l'enjeu, ont compris le message et essaient malheureusement bien trop souvent de présenter leur dossier en fonction des préoccupations des décideurs et en choisissant les représentations du problème les plus susceptibles de retenir leur attention. Ce qui peut faire en sorte que des problèmes peu répandus retiennent l'attention. En effet, la nécessité d'intéresser les bailleurs en invoquant leur image politique pourrait conduire à des politiques publiques qui correspondent davantage aux priorités des donateurs qu'à celles des bénéficiaires. D’où les problèmes d'appropriation de l'aide et de décalage des politiques d'aide par rapport aux besoins des populations.

\section{Pour aller plus loin}

Fassin, D. (200o). Les enjeux politiques de la santé. Études sénégalaises, équatoriennes et françaises. Paris, Éditions Karthala.

HANLON, M. et al. (2014). Regional variation in the allocation of development assistance for health. Globalization and Health, $10: 8$. doi:10.1186/1744-8603-10-8

Jones, B. D. et Baumgartner, F. R. (2005). The Politics of Attention. How Government Prioritizes Problems? The University of Chicago Press. 


\section{En Afrique, les soins gratuits déresponsabilisent la population, ne sont pas valorisés et sont de mauvaise qualité}

Valéry Ridde et Ludovic Queuille

De nombreuses personnes prétendent que la gratuité des soins déresponsabilise les usagers en leur permettant, par exemple, d'abuser des services. Elles avancent aussi que celle-ci les amènerait à ne pas valoriser les services et les produits offerts ou à les considérer comme de mauvaise qualité. Pourtant, de nombreuses données scientifiques montrent le contraire. Certains prétendent aussi que la gratuité des accouchements va entraîner une hausse des naissances et donc que cette mesure va à l'encontre de la promotion de la planification des naissances. Il n'existe aucune preuve pour soutenir une telle idée, alors que de nombreuses connaissances tendent à démontrer le contraire'.

Des recherches sur les traitements contre le VIH mis en place par l'État du Sénégal ont montré que l'instauration de la gratuité a été favorable à la responsabilisation des malades dans le suivi de leur traitement. La lutte contre le VIH est ainsi devenue bien plus efficace. C'est pour la même raison que le traitement contre la tuberculose est gratuit dans de

1. Ce chapitre est composé d'une section d'un article publié: Nine misconceptions about free healthcare in sub-Saharan Africa, Development Studies Research, vol. 1 (1), 2014, Valéry Ridde, Ludovic Queuille et Marame Ndour. 
nombreux pays en Afrique. Des recherches expérimentales ont montré que le fait d'avoir payé ou non les moustiquaires en Ouganda et au Kenya ou du désinfectant pour l'eau en Zambie ne modifie en rien leur niveau d'utilisation par les populations. Ceux qui payent n'utilisent pas plus leur produit que ceux qui en bénéficient gratuitement.

La gratuité permet à plus de malades d'être soignés et guéris, et donc aux citoyens d'être acteurs dans la gestion de leur santé. Si les consultations prénatales $(\mathrm{CPN})$ sont gratuites dans la plupart des pays d'Afrique, c'est pour créer des liens entre les femmes enceintes et les soignants afin d'encourager les accouchements par du personnel qualifié dans les maternités. Ainsi, en Afrique, les femmes qui ont réalisé trois $\mathrm{CPN}$ ont neuf fois plus de chances d'accoucher dans un centre de santé que celles qui n'en ont réalisé aucune. Au Burkina Faso, dans une région où la population est majoritairement pauvre, un an après l'instauration de la gratuité, $80 \%$ des enfants malades ont été conduits dans un centre de santé, alors qu'ils n'étaient que $30 \%$ lorsque les soins étaient payants. Même les parents des enfants malades vivant à plus de $10 \mathrm{~km}$ des centres de santé se déplacent en masse pour bénéficier des soins gratuits ( $77 \%$ de plus qu'auparavant). A contrario, au Rwanda, où les mutuelles de santé réclament encore un paiement au centre de santé, seulement $33 \%$ des enfants malades se rendent dans un centre de santé.

Lorsque l'on supprime le paiement direct au point de service, l'augmentation de la fréquentation des services est immédiate, importante, mais surtout durable lorsque la mise en œuvre ne fait pas défaut. Si les services gratuits n'étaient pas valorisés, la fréquentation s'essoufflerait au fil du temps. Au contraire, la gratuité, lorsqu'elle est bien mise en œuvre et suffisamment financée, permet à un plus grand nombre de malades d'être soignés et guéris, et donc aux citoyens de reprendre confiance dans le système de santé. Et lorsque l'on sait combien la maladie engendre des frais importants en dehors de ceux déboursés au centre de santé, il est peu logique et inconvenant de considérer que les populations dévalorisent les soins qu'ils viennent chercher, sous prétexte qu'ils sont gratuits. Tout ceci est confirmé, par exemple, par une étude sur la qualité perçue des accouchements comparant la perception des femmes ayant accouché dans un district du Burkina Faso où elles doivent payer à celle des femmes du district voisin où la gratuité est expérimentée. Il n'existe pas de différence pour les trois dimensions de la qualité étudiées: relations 
interpersonnelles, soins prodigués et environnement de l'accouchement. Autrement dit, ne pas payer ne dévalorise pas la perception de la qualité des accouchements.

Si l'on en vient maintenant à la gratuité des accouchements en Afrique, c'est dans les milieux urbains qu'ils sont les plus chers et que les taux de natalité sont les plus faibles. Au Mali et au Burkina Faso, les femmes vivant en milieu rural ont respectivement 7,2 et 6,7 enfants en moyenne, alors que celles vivant dans la capitale n'en ont que 4,8 et 3,4. Au Burkina Faso, au Ghana, au Sénégal et dans d'autres pays, la tendance à la baisse de la fécondité se maintient, alors que les accouchements ont été fortement subventionnés ou rendus gratuits dans la période 2005-2010. De plus, on sait depuis longtemps que l'éducation est un des plus importants facteurs associés à la fécondité et que la pauvreté est un frein à sa baisse. Or, les dépenses lors des accouchements, et surtout des césariennes, peuvent entraîner les ménages les moins aisés dans l'extrême pauvreté.

Du point de vue des droits humains mais aussi sur un plan stratégique, le concept de "maternité sans risque " vise à sauver la vie des femmes lors des accouchements assistés par du personnel qualifié en facilitant la détection des complications (survenant dans $15 \%$ des accouchements), leur référence et leur prise en charge à l'hôpital. Or, faire payer les accouchements, c'est favoriser les accouchements à domicile et ainsi restreindre les possibilités de soins dont doit disposer toute femme enceinte. D’une façon générale, il est essentiel de renforcer l'accès des populations à l'information et aux services de santé sexuelle et reproductive pour que les femmes puissent être en mesure de choisir librement d'utiliser un produit contraceptif.

La qualité des soins est un enjeu majeur des systèmes de santé. Certains s'inquiètent des effets négatifs de la gratuité sur la qualité des soins, du fait notamment de l'augmentation du nombre de consultations pour les agents de santé, de la trop grande liberté offerte aux prescripteurs, de ruptures dans les stocks de produits médicaux, etc. Il n'existe pas, à notre connaissance, de recherche établissant un lien direct et quantifié entre la gratuité et la baisse de la qualité des soins. Cependant, des recherches qualitatives montrent, par exemple au Niger ou au Mali, que lorsque les politiques de gratuité des soins sont sous-financées et mal organisées, elles engendrent une baisse de qualité vue sous l'angle de la disponibilité des médicaments. Mais cette mauvaise qualité préexiste très 
souvent aux politiques de gratuité et cette exacerbation s'explique en grande partie par la mauvaise mise en ouvre et non par le principe de gratuité lui-même. A contrario, le principe du paiement par les usagers ne renforce pas forcément la disponibilité des médicaments. Au Burkina Faso, par exemple, les médicaments de lutte contre le paludisme distribués par les agents de santé communautaire restent payants, contrairement au Mali, où ils sont gratuits. Or, les mêmes problèmes de mise en œuvre et de logistique se posent et rendent la disponibilité de ces produits très problématique et inquiétante. La qualité de la mise en œuvre des politiques est donc centrale à l'atteinte de leurs objectifs.

Deux études concernant la qualité des soins dans une situation où la gratuité est bien mise en œuvre et où la fréquentation des services a très largement augmenté ont été réalisées au Burkina Faso. La première montre que, contrairement à cette idée reçue, les durées moyennes des actes réalisés par les agents de santé dans un district où les soins sont gratuits ne sont pas inférieures à celles du district voisin où ils restent payants (respectivement 12 contre 9 minutes pour les consultations curatives, 63 contre 62 minutes pour les accouchements et 15 contre 11 minutes pour les $\mathrm{CPN}$ ). La seconde étude montre que les prescriptions médicales concernant les soins gratuits pour les enfants de moins de cinq ans restent très proches des normes de l'OMS et de l'État. L'importance des mesures d'accompagnement et de la supervision de la gratuité mentionnée dans cette étude est confirmée par une recherche menée au Sénégal, où la distribution gratuite des traitements contre le paludisme par les agents de santé communautaire sans autre mesure pose de sérieuses difficultés.

La gratuité des soins ne résoudra pas tous les problèmes que vivent les populations et les systèmes de santé. Toutefois, le fait même qu'elle agisse souvent comme un révélateur des dysfonctionnements constitue en soi une réelle possibilité d'amélioration des systèmes de santé. Dans cette optique, il parait important de prendre en considération les nombreuses recherches qui montrent que la plupart des idées communément exprimées contre le principe de la gratuité des soins ne reposent pas sur des preuves. La plupart du temps, ces idées reçues sont liées à des contextes où la gratuité des soins n'est pas mise en œuvre correctement, est peu financée et ne constitue pas une réelle priorité politique. Ce n'est donc pas contre le principe lui-même qu'il faut lutter, mais pour son application efficace. En effet, lorsque la gratuité est bien planifiée, suffisamment 
financée et mise en œuvre grâce à des mesures ciblées d'accompagnement, elle s'avère très efficace et équitable.

\section{Pour aller plus loin}

Bates, M.-A., Glennerster, R., Gumede, K. et Duflo, E. (2012). Pourquoi Payer? Field Actions Science Reports [Online], Special Issue 4 | 2012, Online since 31 January 2012, http://factsreports.revues.org/1301.

Gratuité des soins en Afrique subsaharienne: en finir avec certaines idées reçues (2013). http://www.oxfamfrance.org/rapports/acces-sante/gratuite-dessoins-afrique-subsaharienne-idees-recues

RidDE, V. (2012). L'accès aux soins de santé en Afrique de l'Ouest. Au-delà des idéologies et des idées reçues. Montréal, Presses de l'Université de Montréal. 


\title{
Financer le système de santé sur la base des résultats sauve des vies
}

\author{
Katerini T. Storeng
}

L'un des facteurs les plus importants pour expliquer les mauvais indicateurs de santé en Afrique et dans les pays à faible revenu est l'absence ou le retard d'accès à des soins de santé de qualité. Le financement basé sur les résultats (FBR) est actuellement promu comme une des stratégies pour améliorer la santé et les services de santé. Il s'agit notamment d'améliorer l'accès aux services essentiels pour les femmes et les enfants afin d'accélérer la progression vers les Objectifs du Millénaire pour le développement des Nations Unies. Récemment, la plupart des pays donateurs de l'aide (Royaume-Uni, Norvège, Allemagne, Japon, Suède et États-Unis) se sont associés à la Banque mondiale, l'UNICEF, le Fonds mondial de lutte contre le VIH, la tuberculose et le paludisme, l'Alliance GAVI pour la vaccination et la Fondation Bill et Melinda Gates pour soutenir le passage à l'échelle du FBR dans les pays à faible revenu.

Le FBR consiste, par des incitatifs financiers, à payer les centres de santé ou les professionnels de la santé en fonction de leur réussite dans l'atteinte d'un résultat spécifique. Classiquement, le financement est associé à une variété d'objectifs quantitatifs, tels que le nombre d'accouchements assistés ou de vaccinations réalisées. La Banque mondiale et ses partenaires avancent que le «FBR peut permettre d'avoir $20 \%$ de soins de santé en plus pour les mêmes fonds». Selon le président de la Banque mondiale, Jim Yong Kim, les recherches ont montré que le FBR sauve des 
vies et améliore l'accès à la qualité. De même, le ministre des Finances et du Planning économique du Rwanda avance que des évaluations rigoureuses dans son pays «prouvent que le FBR peut être une réussite, quelles que soient les conditions, si nous soutenons son processus». Mais jusqu'à quel point ces affirmations sont-elles fondées sur des preuves?

En 2012, une recherche approfondie et rigoureuse des preuves scientifiques sur les effets du FBR sur la santé a montré que les données probantes à cet égard sont trop faibles pour soutenir l'hypothèse de son efficacité. Elle avance aussi que de meilleures études doivent être entreprises avant de recommander le FBR comme une solution pour le financement de la santé. Bien que d'autres études aient été publiées après 2012, les preuves que le FBR soit un bon système de financement sans ambiguité restent faibles et la plupart concernent l'expérience d'un seul pays (le Rwanda). Dans les prochaines années, des études actuellement en cours démontreront quand, où et comment le FBR est efficace en santé mondiale. En attendant, les résultats utilisés par la Banque mondiale dans ses communiqués de presse proviennent d'évaluations qui sont en cours et celles-ci n'ont pas été analysées par des scientifiques pour en assurer l'intégrité et la qualité. D’ailleurs, un expert remarquait qu'«il est vraiment étrange que la Banque mondiale ait commissionné de très nombreuses évaluations sur ses programmes de FBR, alors qu'elle décide d'affirmer que le FBR fonctionne avant que la plupart des preuves scientifiques de son efficacité soient disponibles».

Même si le FBR semble contribuer à l'amélioration de quelques indicateurs mesurables, au moins à court terme, nous avons très peu de connaissances sur ses effets à long terme ou ses impacts plus généraux sur les systèmes de santé. Un nombre important d'effets inattendus ont déjà été mis au jour, tels que la manipulation des chiffres pour atteindre les résultats et obtenir plus de récompenses, la sélection des patients qui sont plus faciles à traiter et la concentration des activités vers les plus faciles à organiser ou les plus payantes. Bien que les bailleurs de fonds reconnaissent parfois que ce sont des problèmes potentiels associés au FBR, ils sont rarement mentionnés lorsque ce modèle est discuté en public et promu. Des études anthropologiques ont révélé que le FBR peut endommager les relations sociales et la collaboration entre les professionnels de la santé. Ainsi, l'évaluation de cette approche ne doit pas se limiter aux résultats mesurables, mais doit aussi tenter de comprendre comment les résultats sont atteints, et quels sont les effets d'entraînement potentiels du FBR sur 
le système de santé, les relations sociales entre les professionnels de santé ainsi qu'entre ces derniers et les patients.

Le débat académique concernant le FBR est très polarisé. Un expert confirmé en santé mondiale affirmait qu'il était inquiet du fait que même de meilleures données probantes ne changeraient pas les oppositions fondées sur des idéologies contre l'utilisation de l'argent comme incitatif dans le domaine de la santé. D'un autre côté, il est important de reconnaître que le FBR n'est pas non plus neutre idéologiquement. Il est fondé sur une théorie du comportement humain qui n'est pas sans valeur, mais qui s'inscrit dans une perspective plus large en faveur d'une instrumentalisation économique et d'une idéologie néolibérale en santé mondiale. Des arguments discutables ou incomplets concernant les preuves de l'efficacité du FBR contribuent à rendre ses fondements idéologiques obscurs et, ainsi, à affecter une nécessaire discussion plus nuancée à propos de la manière dont les pays à faible revenu doivent financer leur système de santé pour améliorer la santé de leurs citoyens.

Pour les pays à faible revenu, la question centrale aujourd'hui est plus de savoir comment, plutôt que de savoir s'il faut introduire le FBR dans le secteur de la santé. Bien que les bailleurs de fonds avancent que les pays doivent décider ce qu'ils veulent encourager par des mesures incitatives, il ne fait aucun doute que ces bailleurs exercent une pression énorme sur ces pays pauvres et dépendants de l'aide pour qu'ils introduisent ce nouveau modèle, qui est aujourd'hui soutenu par tous ceux qui comptent à l'échelle internationale. Il est donc essentiel de s'engager dans une discussion bien plus nuancée concernant les effets attendus et inattendus de ce modèle et de défier une vision largement sans critique affirmant que le FBR est une approche fondée sur des preuves pour «sauver des vies».

\section{Pour aller plus loin}

Fretheim, A., Witter, S., Lindahl, A. K., et Olsen, I. T. (2012). Performance-based financing in low- and middle-income countries: still more questions than answers. Bull World Health Organ, 9o(8) : 559-559.

Magrath, P. et Nichter, M. (2012). Paying for performance and the social relations of health care provision: An anthropological perspective. Social Science \& Medicine, 75(10): 1778-1785.

Mishra, A. (2014). Trust and teamwork matter: Community health workers' experiences in integrated service delivery in India. Glob Public Health: 1-15. 


\title{
Les médicaments du marché informel sont des faux médicaments
}

\author{
Carine Baxerres
}

Le marché informel du médicament est l'expression généralement choisie en anthropologie pour désigner les pratiques de vente et d'achat de médicaments qui se déroulent hors du cadre formel imposé par l'État. Il s'agit de médicaments qui sont vendus hors des officines, dans les marchés, aux coins des rues, de porte en porte, etc., par des personnes qui ne disposent pas d'une reconnaissance officielle. Dans le langage commun, ces pratiques sont désignées dans les pays francophones d'Afrique par les expressions les pharmacies par terre ou encore les médicaments de la rue.

Ces pratiques ont lentement émergé dans ces pays au cours des années 1950. Mais leur développement massif date de la fin des années 1970, alors que les pays éprouvent des problèmes de gestion pharmaceutique et de ruptures régulières et importantes de médicaments. À partir du milieu des années 1990, les institutions sanitaires de ces pays, tous touchés par la question du marché informel, décident de prendre des dispositions préventives et répressives pour lutter contre celui-ci. Se développe alors progressivement, parmi les acteurs institutionnels (ministères de la santé, ordres des pharmaciens, associations, organisations non gouvernementales et fondations) une opinion radicalement défavorable à ce marché informel, qui s'articule autour de quelques points parmi lesquels se retrouvent les notions de contrefaçon pharmaceutique et de qualité 
douteuse des médicaments vendus. S'instaure dès lors l'idée selon laquelle les médicaments du marché informel sont des faux médicaments.

Progressivement, cette opinion s'est développée à une échelle supérieure. Des institutions investies dans le champ pharmaceutique, elle a atteint le grand public, sphère où elle a pris le caractère d'un discours dominant. Relayée dans les articles de journaux, les émissions et reportages télévisuels et radiophoniques, l'opinion publique internationale est devenue consensuelle sur cette question. La mention du marché informel du médicament comme sujet de recherche, par exemple, recueille inévitablement des propos de la sorte: «Ah oui, les faux médicaments, les petites pilules rouges, bleues, jaunes qui sont vendues sous le soleil en Afrique...» Cette opinion publique se conforte avec l'arrivée sur le devant de la scène internationale de la question de la contrefaçon pharmaceutique et, depuis octobre 2009, avec «l'appel de Cotonou» lancé par Jacques Chirac, de celle des «faux médicaments». Ces questions sont mises en évidence par les institutions sanitaires internationales, les dirigeants de firmes pharmaceutiques, les autorités sanitaires de nombreux pays. Elles sont également relayées par les différents médias.

Pourtant, ce discours est basé sur un amalgame, à la fois non pertinent et non vérifié, entre trois phénomènes distincts: 1) celui du marché informel du médicament, qui pose la question de la légalité des circuits de distribution, 2) celui de la contrefaçon pharmaceutique, qui pose le problème de la légalité des médicaments et des fabricants qui les produisent, et 3) celui de la non-conformité des produits ou de la malfaçon, qui interroge la qualité des médicaments (sous-dosages, surdosages, absence de principes actifs, toxicité). Le terme faux médicaments, d'utilisation plus récente, vient encore brouiller les définitions et renforcer l'imprécision sur les phénomènes qui sont ainsi désignés.

Dans les faits, ces trois phénomènes se prêtent très mal aux évaluations quantitatives. Il est également très difficile d'en apprécier l'impact sur la santé des individus. Leurs effets sur les patients sont difficiles à détecter et à quantifier. Ainsi, la plupart des écrits sur la contrefaçon et les faux médicaments proviennent de journalistes d'investigation locaux et comprennent finalement peu d'investigations scientifiques de santé publique. De telles enquêtes posent d'ailleurs d'infinis problèmes méthodologiques: comment réaliser des investigations réellement représentatives de ces phénomènes? 
Pourtant, à travers les médias, dans les discours des acteurs institutionnels ainsi que dans des articles scientifiques, des chiffres sont avancés. La contrefaçon représenterait $10 \%$ du marché mondial du médicament, $15 \%$ dans les pays développés et plus de $50 \%$ en Afrique et en Asie. Il est également dit fréquemment que ces médicaments sont responsables de 200000 décès par an, sans que l'origine de ce chiffre ne soit réellement explicitée. Le volume du marché informel est également sujet à des évaluations variées. Au Bénin, selon les sources, il représente entre $25 \%$ et $50 \%$ du marché total du médicament, alors que certains professionnels pensent qu'il dépasse le volume du marché formel. Au-delà des chiffres, certains faits divers, tels que celui de faux vaccins contre la méningite introduits au Niger en 1995, sont régulièrement évoqués par différents acteurs, et notamment dans des articles de presse, sans que l'on sache bien s'il s'agit de contrefaçon ou de malfaçon. Dans les faits, il s'agissait de vaccins dans lesquels il n'y avait pas de produits actifs, mais cela n'est que très rarement précisé dans les articles de presse.

Ces trois phénomènes sont ainsi souvent l'objet d'évaluations fantaisistes, extrapolées et pourtant reprises sans trop de précaution dans les discours. Sur le plan international, les médicaments, qui représentent un des secteurs économiques les plus dynamiques et lucratifs, sont l'objet d'enjeux économiques et politiques importants. Les polémiques actuelles autour de la question des brevets pharmaceutiques ainsi que la concurrence croissante entre les firmes pharmaceutiques d'origine occidentale et celles des pays émergents, spécialisées dans la production de médicaments génériques, expliquent en partie la production de ce discours dominant. Les objectifs implicites sont alors loin d'être uniquement sanitaires et de santé publique.

Les recherches que j'ai menées en Afrique de l'Ouest permettent pour le moins de déconstruire l'amalgame entre médicament du marché informel et faux médicament. En effet, une observation participante conduite durant plus de deux ans (2005-2007) dans le centre de la vente informelle de médicaments au Bénin, situé dans le grand marché international de Cotonou, a permis de mettre en évidence deux sources principales d'approvisionnement: 1) les circuits formels du Bénin et des pays francophones voisins (Togo, Burkina Faso, Niger), 2) les circuits formels des pays anglophones voisins, le Nigeria et le Ghana. Dans ces derniers, les médicaments sont achetés auprès de grossistes formels qui distribuent 
des produits autorisés par les agences en charge de la régulation des médicaments. La plupart de ces médicaments ne sont par contre pas autorisés à la vente au Bénin et sont généralement montrés du doigt par les détracteurs du marché informel, comme des faux médicaments.

Il ne s'agit pas de dire que le marché informel n'est pas concerné par la question de la contrefaçon ou de la falsification des médicaments. Plus un marché est soumis à une régulation stricte, moins les risques de circulation de produits frauduleux sont élevés. Mais si des copies frauduleuses de médicaments intègrent le marché informel, elles ne constituent pas - loin de là - la majorité des médicaments qui y sont distribués. Par contre, ces constats mettent en évidence qu'il existe des différences notoires en matière d'approvisionnement pharmaceutique entre d'une part le Bénin et d'autre part le Nigeria et le Ghana. Il s'agit globalement de distinctions entre pays francophones et pays anglophones d'Afrique. Les mêmes médicaments n'y sont pas autorisés et donc distribués légalement. Alors que les approvisionnements pharmaceutiques des pays francophones sont aujourd'hui encore très dépendants des pays occidentaux et particulièrement de la France, les pays anglophones se sont ouverts depuis les années 1970-1980 à des médicaments en provenance des pays émergents d'Asie, et notamment de l'Inde, de la Chine et de l'Indonésie.

Ainsi, plus que la falsification, les questions qui se posent à travers les médicaments vendus dans le marché informel en Afrique concernent l'hétérogénéité des systèmes pharmaceutiques des différents pays et des procédures d'enregistrement des médicaments à l'échelle du continent africain, ainsi que la commercialisation de produits dans un pays où ils ne sont pas enregistrés officiellement.

\section{Pour aller plus loin}

Baxerres, C. (2015). Contrefaçon pharmaceutique: la construction sociale d'un problème de santé publique. In Egrot, M., Desclaux, A. (dir.). Anthropologie du médicament au Sud. La pharmaceuticalisation à ses marges. Paris, L'Harmattan - Editions de l'IRD: 129-146.

Baxerres, C. (2014). Faux médicaments, de quoi parle-t-on? Contrefaçons, marché informel, qualité des médicaments... Réflexions à partir d'une étude anthropologique conduite au Bénin, Bulletin de la société de pathologie exotique, 107: 121-126. http://link.springer.com/article/10.1007\%2Fs13149-014-0354-9\#page-1

Jourdain-Fortier, C. et Moine-Dupuis, I. (2013). La contrefaçon de médicaments: les premiers pas d'une réaction normative internationale. Paris, LexisNexis. 


\title{
Les missions internationales médicales bénévoles à court terme sont efficaces
}

\author{
Stephan Brenner
}

Le fait de s'engager bénévolement dans des missions médicales à court terme est de plus en plus répandu chez les médecins, les internes et les étudiants en médecine de l'Amérique du Nord et de l'Europe occidentale. L'engagement bénévole en médecine répond à la tradition altruiste de la profession médicale qui s'enracine dans l'offre en soins de santé aux plus démunis et aux communautés mal desservies des pays à faible revenu. Cet engagement s'inscrit dans le courant de l'éthique médicale qui prône la responsabilité sociale envers les plus vulnérables et les plus défavorisés. Par ailleurs, dans les pays riches, où la médecine reste un domaine académique compétitif, le bénévolat en médecine devient, de plus en plus, un prérequis intéressant pour étoffer les curriculum vitæ des cliniciens. Avec le manque considérable de ressources humaines dans la plupart des pays à revenu faible, il n'est pas surprenant de constater que de nombreuses organisations non gouvernementales à but non lucratif essaient de répondre à la pénurie locale de médecins généralistes et spécialistes en recrutant des cliniciens en provenance des pays riches.

Les missions à des fins médicales peuvent se regrouper en trois catégories. Il y a d'abord les formes diverses et variées d'opérations de secours humanitaires. Ces missions sont organisées pour secourir un nombre important de victimes après une catastrophe naturelle ou d'origine 
humaine. Elles se déroulent au sein des hôpitaux de campagne et s'appuient sur des équipes d'urgence et chirurgicales expatriées pour répondre aux besoins aigus en soins des populations touchées. La deuxième catégorie regroupe les missions médicales à long terme. Les donateurs, souvent en collaboration avec les gouvernements locaux, recrutent, pour une période allant de plusieurs mois à quelques années, des cliniciens spécialistes expatriés pour travailler dans des centres de santé tertiaires afin de combler le manque de pédiatres, dentistes, ophtalmologistes, anesthésistes sur place. Pour finir, les missions médicales à court terme sont généralement composées de plusieurs médecins, infirmières ou étudiants en médecine qui s'engagent bénévolement sur des périodes de plusieurs jours ou quelques semaines. Ces bénévoles travaillent dans des régions éloignées ou rurales et soignent gratuitement des personnes souffrant de maladies aiguës ou chroniques grâce aux dons de médicaments et de matériel médical.

La controverse au sujet du bénéfice, de l'impact et de l'efficacité des missions humanitaires médicales demeure encore actuelle. Les données probantes à ce sujet sont, malheureusement, rares et insuffisantes. Le manque de pérennité et l'intégration plutôt informelle des missions médicales dans les systèmes de santé locaux sont des préoccupations souvent exprimées. Les missions d'aide humanitaire ont lieu habituellement dans des régions où les structures sanitaires locales ont été détruites ou sont très souvent surchargées. Si on considère la structure opérationnelle mise en place pour répondre à la catastrophe, on remarque que l'intention première n'est pas de programmer des missions à long terme, mais plutôt d'intégrer le système de soins national et local pour mieux gérer le problème sanitaire. Les missions de longue durée, quant à elles, sont généralement initiées et soutenues par les administrations nationales de santé avec le soutien des gouvernements donateurs pour couvrir les besoins en santé de la population locale. Par conséquent, l'intégration et la participation à long terme sont des caractéristiques relativement communes. Toutefois, ce type de missions reflète souvent la négligence de l'État (qui accueille ces aides) envers sa responsabilité réelle d'investissement et d'organisation des services de santé nécessaires à son propre fonctionnement. Quant aux missions à court terme, les principales préoccupations demeurent leur manque de pérennité et d'intégration locale, puisqu'elles fonctionnent souvent avec les systèmes de santé étrangers loin des cadres fixés à l'échelle nationale. Somme toute, les 
missions à court terme restent probablement la forme de bénévolat médical la plus attrayante. Elles sont de courte durée et donc plus conformes aux obligations cliniques qui incombent aux médecins venant de pays étrangers. Elles recrutent volontiers des étudiants en médecine ayant des qualifications professionnelles limitées et sont reconnues comme une riche et unique expérience de vie professionnelle et personnelle auprès des plus démunis (patients et soignants locaux).

Les quelques données probantes disponibles sur l'impact des missions médicales à court terme étudient principalement les répercussions du bénévolat médical sur l'offre en soins cliniques et le système de santé. Les effets négatifs sur la qualité des soins cliniques offerts ont été recensés dans des situations où les besoins de santé locaux ont été négligés ou mal compris. Dans des contextes aux ressources limitées, les prestations de soins requièrent souvent le maintien des approches thérapeutiques habituelles. En effet, les meilleures stratégies curatives possibles dans un pays développé ne peuvent pas toujours être applicables dans un pays aux ressources médicales limitées. Le protocole médical standard en vigueur dans les pays riches exige souvent un suivi médical, des traitements postchirurgicaux, des mesures de réhabilitation ou encore d'autres formes d'interventions cliniques multidisciplinaires. Élaborer des prestations de soins qui ne sont pas adaptées aux directives locales ni à la réalité des établissements de santé locaux (couramment surchargés) ne s'inscrit en rien dans une perspective de pérennité puisque, une fois la mission terminée, le suivi par le médecin expatrié n’est pas souvent envisageable. Si les patients, aux ressources financières déjà limitées, désirent continuer le vaste processus de soins mis en place par les missions humanitaires, ils devront engager des dépenses supplémentaires.

Une autre préoccupation sur la qualité des soins reliée à l'éthique médicale mérite d'être soulevée. Travailler dans un établissement de santé avec relativement peu de supervision permet - et parfois on s'y attend aux internes expatriés en médecine et même aux étudiants en médecine d'exécuter des tâches ou des procédures cliniques pour lesquelles ils ne sont pas pleinement qualifiés. De même, les médecins cliniciens expérimentés pourraient effectuer des procédures cliniques, qui ont été remplacées dans les pays riches par des techniques modernes de diagnostic moins invasives, sans jamais les avoir réalisées régulièrement dans leur pratique professionnelle. Les ressources en matériel médical limitées, le manque 
de supervision et l'absence d'un cadre déontologique réglementaire pour encadrer les pratiques professionnelles abusives pourraient finalement compromettre la sécurité des patients. Enfin, en ce qui concerne l'organisation du travail, la maîtrise insuffisante des langues et des coutumes locales requiert souvent que les infirmières et les assistants médicaux locaux endossent le rôle d'interprète entre le bénévole et le patient. Cette pratique empêche les agents de santé locaux d'accomplir leurs tâches quotidiennes et les force à devenir de simples auxiliaires.

Selon une perspective de santé publique, les effets des missions médicales à court terme semblent encore plus problématiques. Si on tient compte d'une approche coûts-bénéfices, les coûts par cas traité lors d'une mission à court terme sont plus importants que pour toute autre intervention de soins de santé offerte. Le bénévolat représente un coût financier élevé, si on tient compte de toutes les dépenses qu'il faut prévoir : les billets d'avion, les vaccins et l'hébergement. De plus, les impacts sur la santé des interventions médicales offertes lors de missions sont de très courte durée dans des contextes de soins où, généralement, la malnutrition, le manque d'hygiène et les difficultés à contrôler le vecteur de la maladie prévalent. Ainsi, l'allocation des ressources financières et humaines lors des missions humanitaires médicales à court terme tend à être extrêmement inefficace. Comme la plupart des missions médicales se centrent principalement sur les interventions curatives, les besoins réels des communautés locales en matière de santé - les mesures de prévention des maladies, le dépistage de la maladie, l'éducation à la santé - et les problèmes des systèmes de santé locaux - la mauvaise qualité des infrastructures de santé, la corruption, le manque de professionnels de santé - sont trop souvent négligés. Malgré le nombre de missions à court terme qui ont été organisées et financées avec le soutien des pays riches, seulement quelques-unes ont su collaborer et créer des accords avec les administrateurs de santé locaux. Ce manque de collaboration conduit souvent à la duplication des programmes nationaux de santé, au gaspillage des ressources et à la négligence de l'importance du rôle des autorités locales. Dans leur désir de porter secours aux communautés marginalisées ignorées des politiques de santé déjà existantes, les missions médicales bénévoles ont plutôt contribué à perturber les systèmes de santé.

Les soins de santé offerts lors des nombreuses missions à court terme, empreintes d'altruisme et de bienveillance, sont-ils réellement de bons 
soins? Les structures opérationnelles actuelles des missions médicales à court terme contribuent-elles efficacement à relever les défis des insuffisances des infrastructures de santé en soulageant durablement les systèmes de santé surchargés et en allégeant le fardeau de la maladie des populations frappées par la pauvreté?

Les communautés locales considèrent généralement les missions médicales comme un signe visible d'une solidarité internationale. Les professionnels de santé locaux apprécient généralement la possibilité de pouvoir mesurer leurs connaissances et compétences professionnelles en travaillant aux côtés de leurs collègues des pays riches. Néanmoins, les données probantes qui montrent l'impact positif des missions médicales à court terme sur la santé semblent être contrebalancées par la présence importante des conséquences négatives. Il semble finalement que les missions médicales servent avant tout aux médecins des pays riches dans leur recherche de moyens aventureux pour combiner les soins cliniques avec la philanthropie. Les concepts de missions humanitaires médicales nourris d'une approche plus bidirectionnelle peuvent être une solution envisageable pour répondre à certaines des critiques soulevées. Toutefois, de tels concepts devraient requérir une évaluation critique du potentiel réel des missions médicales à fournir des soins de santé efficaces et durables.

\section{Pour aller plus loin}

Chevalier, A. et al. (2002). Analyse des conditions préalables à la mise en œuvre de formations en santé dans le cadre de l'aide humanitaire. Santé publique, 14: 37.

MAKI, J. et al. (2012). Health impact assessment and short-term medical missions: a methods study to evaluate quality of care. BMC Health Service Research, 8: 121.

Matiniuk, A. L. C. et al., Brain Gains. A literature review of medical missions to low and middle-income countries. (2012) BMC Health Service Research, 12: 134. 


\title{
7
}

\section{Le VIH/sida pose des questions totalement inédites aux politiques de santé}

\author{
Laurent Vidal
}

Le virus de l'immunodéficience humaine (VIH en français), cause du sida, a été découvert il y a 30 ans. L'épidémie qui s'est développée dans le monde est singulière notamment par ses incidences variées. Le VIH a alors frappé les esprits pour différentes raisons: ses caractéristiques virologiques, sa large diffusion mondiale, sa transmission par voies sexuelle, sanguine et de la mère à l'enfant et, pendant longtemps, son caractère mortel. La communauté internationale, les États et le monde associatif se sont alors mobilisés pour développer des stratégies de dépistage et de prévention, mettre au point et diffuser des traitements de plus en plus efficaces.

Partant de là s'est construit le raisonnement suivant: les caractéristiques du VIH sont originales et l'épidémie que ce virus a engendrée est particulière, donc les questions que l'épidémie pose à la société, aux individus et aux politiques de santé sont singulières et inédites. Il convient par conséquent d'inventer des solutions aux problèmes posés qui s'avéreraient donc inédites.

Le VIH/sida poserait donc des questions totalement inédites aux politiques de santé. Ainsi, l'épidémie de VIH aurait «donné naissance à la décentralisation des soins», "lancé le principe de la gratuité des soins» et «créé la stigmatisation du malade». Or, si l'épidémie de VIH a pu leur 
conférer une forme particulière, voire originale, nous n'avons là ni des idées, ni des actions, ni des situations nouvelles.

Très tôt, l'épidémiologie du VIH et les études sur sa répartition spatiale ont montré, par exemple en Afrique, que l'infection se diffuse aussi bien en milieu rural qu'en milieu urbain, notamment à partir de grandes voies de communication. Par ailleurs, les systèmes de santé sont très souvent organisés de façon pyramidale, des structures de santé «de base» aux grands centres hospitaliers universitaires (CHU), en passant par les structures départementales et régionales. Les $\mathrm{CHU}$ sont présentés comme les fleurons de la santé. Ils concentrent les spécialistes et forment les futurs médecins. Ils attirent aussi nombre de patients qui pourraient être traités dans des structures périphériques. Avec l'arrivée de l'épidémie de VIH, ces $\mathrm{CHU}$ ont été logiquement en première ligne, leurs services de maladies infectieuses hospitalisant les patients (et développant des projets de recherche). Donc, plus que pour toute autre pathologie, la prise en charge du VIH, à ses débuts, a renforcé le pouvoir, le rôle et l'attractivité des CHU. Compte tenu de la réalité de la diffusion spatiale du VIH, a émergé un discours sur la nécessité de « décentraliser la prise en charge». Les bailleurs de fonds ont soutenu la création de comités locaux de lutte contre le sida (par exemple au Cameroun), représentant la structure centrale de coordination de la lutte contre le sida. Par ailleurs, les responsables de la santé des pays concernés ont progressivement décidé de rapprocher les traitements des malades. Ils ont fait en sorte que les centres de santé au niveau départemental dépistent le VIH, éventuellement mettent sous traitement les patients et les suivent. Pour ce faire, des services ont été créés au sein de ces centres de santé. Nous voyons donc que le dispositif général de proximité des structures de santé préexistait à la décision de décentraliser la prise en charge du VIH. Et ce depuis de nombreuses années. Il n'est qu'à se rappeler de la médecine mobile du docteur Jamot au début du siècle, durant la colonisation française de l'Afrique. Plus récemment, d'autres "problèmes de santé» ont donné lieu à des politiques de décentralisation. Citons par exemple le cas de la tuberculose, dont la prise en charge s'est décentralisée avant celle du VIH, au point d'ailleurs de lui servir de modèle. En somme, la «décentralisation de la prise en charge du VIH» n'est ni une décision politique ni une action originales. Dire le contraire, c'est estimer, par une déduction arbitraire, que, sous prétexte que l'épidémie de VIH a des spécificités, elle a généré des stratégies radicalement nouvelles. 
L'accès aux traitements du VIH a engendré une forte mobilisation des activistes de la lutte contre le sida comme des chercheurs, relayés par les décideurs locaux de la santé, dans deux grandes directions. D’une part, cela a consisté à faire en sorte que les traitements disponibles au Nord le soient aussi au Sud, où se trouve l'immense majorité des personnes vivant avec le VIH. D’autre part, une fois les médicaments présents, il s'agissait d'œuvrer pour des prix en baisse, voire d'obtenir leur gratuité. Cela fut le cas dans un nombre croissant de pays, à partir du tout début des années 2000. La mobilisation dans les pays, et à l'échelle internationale, a certainement été inédite et sans commune mesure dans la santé publique. Mais, si nous avons là des avancées majeures dans la lutte contre le sida, nous n’avons pas là une stratégie de santé publique nouvelle. L'épidémie de VIH n'a inauguré ni l'idée ni la réalité de la gratuité d'un traitement. Les traitements de la tuberculose et la vaccination sont gratuits depuis longtemps. Là encore, le recul historique est important. Depuis un siècle, les décideurs de la santé comme les médecins attirent régulièrement l'attention sur la question des coûts de traitements et de la nécessité d'en faciliter l'accès, y compris via leur gratuité. Une question ancienne et que n'a pas inaugurée l'épidémie de VIH, même si le processus ayant débouché sur la mise à disposition gratuite des traitements y a pris des formes spécifiques.

Par ses modes de transmission, par ce à quoi elle renvoie (la sexualité), mais aussi par son absence de traitement pendant plus de 15 ans, l'infection à VIH a créé des peurs et en a nourri d'autres (celle de l'étranger, par exemple). Peurs intimement associées à des mécanismes de stigmatisation à l'encontre de la personne perçue comme contagieuse ou jugée comme coupable de sa contamination. Des pratiques de stigmatisation et des craintes de celle-ci se sont donc développées simultanément. Or, ni le principe ni la mécanique de la stigmatisation de la personne infectée par le VIH ne sont réellement nouveaux. La lèpre, la tuberculose, voire la maladie mentale, et plus anciennement la peste ou le choléra sont autant de maladies qui ont généré à la fois de la mise à l'écart, de la désignation, du confinement, de l'exclusion et la peur de ceux-ci. De plus, que les cas de stigmatisation liée au VIH aient été plus dénoncés que ceux dont sont victimes d'autres malades ne dit rien sur l'ampleur particulière du phénomène et encore moins sur son caractère nouveau. Affirmer le contraire relève d'une forme de surenchère qui, en construisant de fausses 
spécificités du VIH, pense pouvoir proposer des réponses nouvelles. Or, lutter contre la stigmatisation c'est, et cela a toujours été, pour toutes les maladies, lutter contre les idées reçues sur le risque de contamination, sur la faute individuelle, etc.

«Le VIH/sida pose des questions totalement inédites aux politiques de santé» est une idée reçue fondée sur une compilation d'idées reçues: à la fois l'histoire des politiques de santé et leur actualité l'attestent. Dévoiler cette idée reçue pose aussi la question de l'innovation dans les réflexions, comme dans les actions. Comment la définir, comment la délimiter? Faute d'y réfléchir, les chercheurs reproduiront des schémas erronés, non fondés empiriquement, d'une connaissance toujours nouvelle, de savoirs inédits, alors qu'ils progressent aussi et surtout par affinement, nuances, capitalisation et non uniquement par de grandes ruptures.

\section{Pour aller plus loin}

Fabre, G. (1998). Épidémies et contagion. L'imaginaire du mal en Occident. Paris, PUF. FArmer, P. (1996). SIDA en Haïti. La victime accusée. Paris, Karthala.

VIdal, L. (2000). Femmes en temps de sida. Expériences d'Afrique. Paris, PUF. 


\title{
Les conflits d'équipes sont essentiels à la performance des professionnels de la santé
}

\author{
François Chiocchio, Marie-Hélène Chomienne \\ et Karine Savaria
}

L'imaginaire collectif semble épris de l'idée selon laquelle la confrontation des opinions serait un mécanisme générateur de performance, de créativité et d'innovation. Les chercheurs de plusieurs domaines se sont intéressés à cette question. Or, bien que les résultats de recherches soient probants, il apparaît que la complexité de la question et l'attrait de conclusions simplistes persistent. Nous entreprenons de clarifier la question en suivant une structure historique de l'évolution récente de la recherche sur les conflits'.

Depuis le début des années 1990, les conflits font l'objet de plusieurs études à la suite de la publication d'un résultat de recherche inusité. En effet, initialement, on a trouvé que les conflits étaient reliés positivement à la performance des équipes. L’idée selon laquelle les discussions que provoquent les confrontations engendrent des remises en question du mode de fonctionnement des équipes devint séduisante; mais est-ce vrai dans tous les cas?

Dans le domaine de la santé, diverses études se sont penchées sur le travail des équipes médicales, le plus souvent celles œuvrant en salles

1. Le fonds de démarrage de l'École de gestion Telfer de l'Université d'Ottawa a rendu possible la publication de ce chapitre. 
d'opération ou dans le cadre de traumas, rencontrant des défis particuliers. Formées sur le terrain, ces équipes multidisciplinaires travaillent dans un contexte d'urgence où les conflits sont notoires. Ces études sur les soins primaires mettent en évidence le fait que les conflits sont inévitables et insistent en amont sur l'importance de former les professionnels de santé à la pratique collaborative et aux processus de résolution des conflits. Or, cela n'explique pas pourquoi les conflits seraient profitables dans des contextes d'offre de soins.

Pour cela, il faut développer la notion de conflit. Les chercheurs en psychologie du travail qui s'intéressent aux conflits définissent généralement le terme comme un désaccord perçu ou réel sur le plan des opinions. On les classe en deux catégories: les conflits de tâches et de processus, concernant la nature du travail, et les conflits relationnels, mettant en jeu les émotions. Une grande quantité de recherches ont été réalisées, à tel point que, depuis une décennie, il est maintenant possible d'effectuer des analyses sophistiquées à partir des études initiales. Ainsi, les métaanalyses faites à partir de résultats cumulés de milliers d'équipes de travail, révèlent des tendances intéressantes, mais complexes. Le lien entre les conflits et la performance semble dépendre de plusieurs facteurs, dont notamment les types de conflits en jeu ou encore le type d'équipe dans lequel le phénomène est observé.

\section{L'effet du type de conflit et du type d'équipe}

Ces méta-analyses montrent que les conflits relationnels affectent toujours négativement la performance des équipes. Lorsque les discussions s'enflamment et mettent en jeu des émotions négatives (frustrations, agressivité verbale, ressentiment, par exemple), les effets sont fortement contre-productifs. Dans un contexte de santé, il persiste encore des guerres de territoire et des cas d'agressivité verbale ou de commentaires dérogatoires qui influencent négativement les relations de travail. Les situations de stress associées à la gestion de cas complexes et à l'urgence des tâches sont souvent mises en cause pour expliquer ces comportements. Cela a des répercussions directes et profondes sur les dynamiques fonctionnelles et sur la performance de l'équipe.

Mais que dire des autres formes de conflits? Le seul fait d'éviter les discussions d'affects négatifs suffit-il pour maximiser la performance, la 
créativité et l'innovation? Les recherches se sont donc poursuivies sur deux fronts pour clarifier cette question. D’abord, à la performance se sont ajoutés d'autres éléments importants du succès des équipes, soit la satisfaction des coéquipiers au regard du travail et de leur place dans l'équipe.

On sait comment la satisfaction au travail des professionnels de la santé (médecins, infirmiers et infirmières, psychologues, ergothérapeutes, par exemple) est importante. Ainsi, les recherches ont commencé à révéler un lien négatif entre toutes les formes de conflits et la performance ou la satisfaction. Ces résultats ont été obtenus en tenant compte d'un facteur additionnel, soit le type d'équipe. Il en ressort une relation fortement négative dans les équipes dites complexes et relativement négative dans les équipes dites simples.

On sait bien sûr que les équipes de santé sont complexes. Dans plusieurs pays, la pratique collaborative a été mise de l'avant comme outil essentiel pour améliorer la performance des systèmes de santé. On a ainsi vu la juxtaposition de diverses professions devant travailler ensemble de manière plus collaborative. La difficulté à comprendre les rôles nouveaux que cela entraîne a souvent été soulevée comme source de conflits. Les champs de pratique, et à qui revient la responsabilité ultime du patient, sont souvent mal reconnus, mal définis, mal compris et entravent non seulement la performance de l'équipe, mais aussi la satisfaction du personnel à s'épanouir au sein de l'équipe. Nous savons que mettre en commun des personnes aux connaissances, expériences et spécialités très variées afin de répondre à des problèmes de santé toujours plus compliqués auprès de populations fragiles et grandissantes est loin d'être simple. Mais les recherches sur les conflits n'ont pas nécessairement porté sur les équipes de santé de façon isolée. Malheureusement pour notre propos, les chercheurs ont catégorisé les équipes à l'aide d'autres critères: les équipes de projet et les équipes décisionnelles mixtes (c'est-à-dire les équipes composées de membres variés dont la principale tâche consiste à analyser des informations complexes afin de prendre une décision).

Sans avoir nécessairement effectué de nombreux travaux sur les équipes de santé, il est possible de faire des recoupements intéressants. Par exemple, bien que par équipe de projet on entende souvent des équipes qui œuvrent dans le domaine de l'ingénierie et de la construction, on sait qu'il y a tout de même des équipes de projets dans le domaine de la santé. 
Il s'agit d'équipes de santé qui, en plus de leurs tâches quotidiennes associées aux soins, ont des responsabilités additionnelles, comme la création, la validation et l'implantation de nouvelles manières de fournir les soins. Une fois les procédures implantées au sein des organisations, ces équipes se dissolvent. Ces projets sont exigeants parce que le travail y opère selon une logique différente de celle des soins. De plus, comparativement au travail de fourniture des soins, les professionnels de la santé ne sont pas formés à la gestion de projet. Dans ces contextes, tous les types de conflits sont fortement néfastes et nuisent au succès des projets et à la satisfaction des coéquipiers.

Les équipes décisionnelles sont aussi considérées comme complexes. Ces équipes ont comme tâche principale de cumuler des informations diverses et de les analyser afin de prendre une décision qui règlera par la suite le travail d'autres personnes ou le leur. Les membres chargés d'effectuer une enquête publique (par exemple dans le cas d'un scandale politique) constituent de telles équipes. Des études récentes au sujet d'équipes décisionnelles montrent qu'elles bénéficient de conflits portant sur la nature du travail. Peut-on tirer des parallèles avec les équipes médicales?

On sait bien sûr que les diagnostics et perspectives de traitement élaborés entre médecins, personnel infirmier et autres professionnels de la santé (psychologues, travailleurs sociaux, par exemple) représentent un processus décisionnel complexe, et à ce titre, on peut peut-être penser que des conflits sur la nature du travail sont bénéfiques aussi. Il convient tout de même de se demander pourquoi un tel résultat serait pertinent. Il est possible que le type de résultat attendu autre que la performance et la satisfaction puisse jouer un rôle. En effet, qu'en est-il du rôle des conflits sur d'autres éléments importants, comme la créativité au travail et l'innovation?

\section{Conflits, créativité et innovation}

La créativité fait référence au processus de génération d'idées nouvelles. Une équipe où règne l'ouverture, un sentiment de confiance et de sécurité psychologique (c'est-à-dire où il est permis de s'exprimer sans crainte de représailles ou de railleries), générera plus d'idées nouvelles. La créativité est une composante essentielle à l'innovation. L'innovation fait référence à l'initiation ou à la découverte d'une nouvelle technologie, d'un processus 
ou d'une idée souvent suivie de son développement et de son implantation. Les innovations varient sur le plan de leur caractère radical, novateur et aussi sur le plan de leurs effets. Les innovations sont habituellement le résultat du travail d'équipes de projets. Dans ce contexte particulier, les études laissent entrevoir qu'une certaine quantité de conflits sur la nature du travail serait bénéfique à la créativité et à l'innovation.

En somme, il faut comprendre qu'il y a différents types de conflits: les conflits de tâches et de processus, qui concernent la nature du travail et les conflits relationnels, qui mettent en jeu les émotions. Les conflits au sein des équipes de travail sont inévitables, mais pas nécessairement essentiels. De plus, les conflits sont peu étudiés dans le contexte particulier des équipes de professionnels de la santé qui offrent des soins de manière continue, comme une équipe de santé familiale ou une équipe de soins intensifs. Une certaine quantité de confrontations sans composante affective est pertinente lorsque des décisions doivent être prises dans un contexte qui requiert de la créativité et de l'innovation, mais pas dans les contextes habituels. Enfin, ce qui détermine le succès des équipes n'est pas la présence de conflits, mais la capacité des coéquipiers à les gérer.

\section{Pour aller plus loin}

Beaulieu, S. et Malo, L.A. (2007). Savoir innover en équipe. Montréal, Isabelle Quentin Éditeur.

De Bono, E. (2007). Conflits: comment les résoudre. Paris, Eyrolles.

FURLONG, G. T. (2010). The conflict resolution toolbox: Models and maps for analyzing, diagnosing, and resolving conflict. Mississauga,Wiley. 


\title{
9
}

\section{Le syndrome d'épuisement professionnel du soignant n'existe pas en Afrique}

\author{
Josiane Tantchou
}

Le syndrome d'épuisement professionnel du soignant est largement connu aujourd'hui. Les premiers articles qui l'évoquent sont parus au milieu des années 1970. Pourtant, ce n'est qu'au début des années 1980 que les premières données issues de travaux de recherches empiriques sont publiées. Maslach et Jackson le définissent comme un syndrome d'épuisement émotionnel, de dépersonnalisation et de réduction de l'accomplissement personnel qui apparaît chez les individus impliqués professionnellement auprès d'autrui. L'épuisement émotionnel renvoie au manque d'énergie, à la dépersonnalisation, au développement d'attitudes détachées, négatives, cyniques envers les personnes dont on s'occupe, et la réduction de l'accomplissement personnel traduit le déclin du sentiment de succès et de compétence dans le travail, la diminution de l'estime de soi et du sentiment d'auto-efficacité. Les causes potentielles du syndrome d'épuisement professionnel incluent: l'absence de reconnaissance, les conditions inadéquates de travail, l'absence d'équité, les conflits de valeurs, les conflits/ ambiguités de rôle, la charge de travail, le manque de soutien social, l'impossibilité de contrôler son activité, la sédentarité du travail.

Dans cette perspective, les recherches empiriques se sont principalement focalisées sur les facteurs liés à l'environnement de travail: satisfaction au travail, stress lié à la charge de travail, conflits de rôle, relations 
entre collègues, relations avec les clients, etc. Mais la plupart des travaux se sont focalisés sur l'Amérique du Nord et l'Europe. En Afrique, plusieurs études relèvent une crise des ressources humaines en santé particulièrement aiguë, laquelle a d'importantes répercussions sur les conditions de travail et la charge de travail de certaines catégories de soignants. D’autres travaux ont mis en évidence l'environnement difficile au sein duquel évoluent les soignants, environnement perçu comme une source d'épuisement professionnel. Mais les soignants africains sont-ils affectés par ce syndrome?

Notre réflexion est basée sur des données recueillies dans le cadre d'une étude menée dans le Grand Nord Cameroun, dans un hôpital public de capacité moyenne (150-200 lits).

\section{Un environnement professionnel accablant}

Avec la récession économique du début des années 1990, le Cameroun a vu ses indicateurs sociosanitaires se dégrader de manière importante. Le budget de la santé publique a subi une réduction progressive. Les recrutements se sont arrêtés à la fin des années 1980 pour toutes les catégories de professionnels de la santé, mis à part les médecins. Les postes libérés du fait des départs en retraite et des décès n'étaient pas renouvelés. Au milieu des années 2000, on a estimé qu'un recrutement de 31 ooo personnes était nécessaire pour résorber l'insuffisance numérique. En 2002, 2004 et 2007 , des recrutements spéciaux, insuffisants, ont eu lieu dans le cadre de programmes internationaux de remise de dettes. À cette insuffisance numérique s'ajoute un sous-équipement chronique. Le Cameroun dispose de plus de 1500 structures sanitaires, pour la plupart sous-équipées. Ceci concerne les éléments facilitant les activités routinières de soins aussi bien que ceux qui sont nécessaires à la vie quotidienne sur les lieux de travail (eau courante, eau potable, W.C., papier hygiénique, ordonnanciers, stylos à bille, etc.). Ces conditions inadéquates de travail évoquées comme facteur d'épuisement professionnel ont un impact sur la fluidité des soins et la satisfaction des soignants. S’y ajoute la précarité caractéristique de la fonction soignante dans ce contexte.

Les soignants se distinguent en fonction de leurs statuts: fonctionnaires, fonctionnaires de pays pauvres très endettés (PPTE ), bénévoles et autres, tous précaires. Prenons par exemple le cas des fonctionnaires. 
Ce statut est attesté par l'affectation d'un numéro matricule qui déclenchera le paiement du salaire. Mais le processus n'est pas automatique. Ainsi, un médecin attendra une moyenne de deux années avant de recevoir son premier salaire. Une fois le statut acquis, les soignants doivent avancer en grade. Ici encore, ils devront subir les effets pervers du système administratif, amplifiés pour ceux qui exercent dans les localités éloignées du centre de décision. Les dossiers sont en effet examinés à Yaoundé, la capitale. Les services du courrier dans les administrations publiques n'étant pas fiables, il est conseillé de porter son dossier soi-même aux bureaux qui en ont la charge. Une fois enregistré, le dossier est examiné par différents services. La durée du passage d'un service à un autre dépendra largement de la capacité du fonctionnaire à mobiliser ses réseaux sociaux et ses ressources financières. Si les fonctionnaires ont chacun une expérience unique de ce rapport au service public, on retrouve partout la même trame: les dossiers avancent quand on connaît quelqu'un ou que l'on donne quelque chose. Or, la crise économique et les Programmes d'ajustement structurel (PAS) ont entraîné une baisse des salaires. Ceux-ci ont été revalorisés au début des années 2000 ; cependant, ils restent insuffisants (autour de 140 ooo FCFA [213, $43 €$ environ] pour un généraliste en début de carrière). Cette précarité incite les médecins hospitaliers à donner des consultations dans des cliniques privées, des cours dans les écoles de formation, à assister aux séminaires organisés par diverses organisations menant ou finançant des projets de santé publique dans la région. Pour quelques jours de séminaire, les indemnités dont bénéficient les participants sont parfois nettement supérieures à leurs revenus mensuels (quand ils ont déjà un salaire). Ainsi, les consultations de médecins sont-elles parfois suspendues pendant plusieurs jours. Ces éléments sont pour les soignants les signes du désintéressement de l'État quant à leurs fonctions, donc une absence de reconnaissance symbolique, et d'une absence d'équité notamment dans l'attribution des avancements en grade, facteurs favorisant l'émergence du syndrome d'épuisement professionnel.

L’environnement professionnel étudié est aussi caractérisé par divers type de tensions: tensions entre soignants et soignés, tensions entre soignants liées à la vente illégale de médicaments, aux soupçons et aux accusations de corruption. Si, jusqu’au début des années 2000, la plupart des soignants n'osaient pas évoquer la pratique si ce n'est avec embarras, 
plusieurs, sans aucune distinction d'origine et de statut, abordent aujourd'hui la question parfois délibérément et sans gêne particulière. Ils justifient la pratique et son inéluctabilité. Toutefois, les pratiques de corruption, en dépit de leur généralisation, posent des problèmes de conscience, des conflits de valeurs. Certains soignants aujourd'hui corrompus, reconnaissant «que ce sont des choses qu' ils ne devraient pas faire», rappellent la droiture caractéristique des débuts de leur trajectoire professionnelle, droiture mise à mal par l'attitude des collègues. Il reste des soignants dont l'intégrité est reconnue. Mais celle-ci s'avère fragile, compte tenu de ce contexte. Ainsi enregistre-t-on parfois des réactions de colère et de dépit. Tous ces éléments, auxquels s'ajoute la sédentarité du travail, viennent composer des environnements socioprofessionnels accablants.

\section{Les soignants sont-ils pour autant affectés par le syndrome d'épuisement professionnel?}

Les données indiquent des niveaux d'épuisement émotionnel et de dépersonnalisation bas et un accomplissement personnel modéré pour l'ensemble du personnel. Ces résultats ne permettent pas d'établir l'existence d'un syndrome d'épuisement professionnel. On note un niveau de dépersonnalisation bas dans l'ensemble, un taux d'épuisement émotionnel modéré chez les médecins, bas pour les infirmiers et autres; un accomplissement personnel modéré chez les médecins et les infirmiers et bas chez les autres. Ces résultats sont surprenants compte tenu du contexte, mais aussi du fait des travaux de recherche déjà évoqués. On serait plutôt en présence de soignants frustrés et d’un système de santé à bout de souffle.

\section{Pour aller plus loin}

Maslach, C. et Leiter, M. P. (1997). The truth about burnout. How organizations cause personal stress and what to do about it. San Francisco, Jossey-Bass.

Schaufeli, W. B., Maslach, C. et al. (dir.) (1993). Professional burnout: Recent development in theory and research. Philadelphia, PA, Taylor \& Francis.

Truchot, D. (2004). Épuisement professionnel et burnout. Concepts, modèles, interventions. Paris, Dunod. 


\section{LA PRÉvention et Les SOINS}





\title{
Les génériques ne sont pas aussi efficaces que les médicaments de marque
}

\author{
Joseph Josy Lévy, Amara Bamba et Elhadji Mamadou Mbaye
}

Les médicaments, basés sur des innovations de l'industrie pharmaceutique, demandent des investissements importants. L'obtention de brevets garantit la propriété intellectuelle et la commercialisation pendant 15 à 20 ans. Par la suite, des compagnies peuvent mettre sur le marché des génériques remplaçant les médicaments originaux. Produits à un coût moins élevé, jusqu’à $45 \%$, ils aident à réduire les dépenses de santé. Malgré cet avantage, les idées reçues à leur sujet limitent la progression de leur diffusion et de leur acceptation. Ce serait ainsi des médicaments peu efficaces, de moindre qualité, plus dangereux et d'ailleurs plus chers que les médicaments originaux. Déconseillés dans le traitement de certaines maladies, ils prendraient plus de temps à agir et auraient des effets secondaires importants. Leur bioéquivalence serait trompeuse et le contrôle exercé par les instances de santé moins draconien que pour les autres médicaments. Les installations permettant leur fabrication ne correspondraient pas non plus aux normes de qualité, en particulier dans la zone asiatique. Mais jusqu'à quel point ces idées reçues sont-elles partagées par les groupes d'acteurs impliqués dans la chaîne des médicaments (médecins, pharmaciens, patients)?

Les médecins sont partagés quant au recours aux génériques, variable selon les pays européens et asiatiques. Si la majorité des praticiens européens 
considèrent que les génériques ont les mêmes caractéristiques que les médicaments de marque en termes de qualité, de sécurité et d'efficacité, l'ampleur de leur prescription varie en fonction des demandes des patients. Dans d'autres pays, l'acceptation est plus faible et les réticences sont liées aux problèmes de sécurité des produits locaux et de bioéquivalence avec les médicaments de marque.

Chez les pharmaciens, selon des études menées dans plusieurs régions du monde, les incertitudes entourant les génériques semblent moins marquées. En Asie, les pharmaciens peuvent avoir de bonnes connaissances et des perceptions positives quant à leur sécurité, leur efficacité et leurs avantages économiques, mais ils sont plus divisés quant aux effets secondaires et à la prescription des produits locaux, considérés comme de moindre qualité. La bioéquivalence reste un problème récurrent. En Australie et en Nouvelle-Zélande, les pharmaciens sont, pour la très grande majorité, enclins à les recommander comme produits de substitution. Si, à leurs yeux, leur niveau de sécurité rejoint celui des médicaments de marque, ces derniers sont considérés comme de meilleure qualité et plus efficaces. Cependant, ce sont surtout les avantages économiques des génériques qui sont soulignés. Au Portugal et en France, la grande majorité des pharmaciens ont confiance dans les génériques et sont favorables à leur utilisation, mais ils soulignent quand même quelques effets secondaires importants.

Du côté des patients, les enquêtes suggèrent que les génériques sont aussi associés à des perceptions problématiques et contradictoires, ce que montrent plusieurs études menées en Europe et aux États-Unis. Si, dans plusieurs pays, les génériques ne posent pas de problèmes particuliers et sont bien acceptés, dans d'autres régions, on constate la présence de connaissances incomplètes s'appuyant sur une méfiance qui les associent à des contrefaçons, à une production étrangère et remettent en question leur efficacité. Les génériques seraient moins puissants que les autres médicaments, nécessitant l'absorption de doses plus élevées et impliquant plus d'effets secondaires. Leur prix bas contribuerait à les associer à des produits d'une qualité douteuse, mais l'inverse est aussi vrai. Ces perceptions peuvent varier selon le lieu de résidence, l'origine ethnoculturelle et le niveau socioéconomique.

Comment les instances d'évaluation et de contrôle des médicaments se situent-elles face à ces idées reçues? Une fois de plus, même à ce niveau, 
des réticences liées au processus de production et aux résultats des recherches scientifiques sont relevées, surtout dans le contexte français. Pour l'Académie nationale française de médecine, la bioéquivalence ne serait pas totalement démontrée. Les contraintes liées à l'évaluation de la qualité et à l'inspection, à la multiplication des intermédiaires et à la progression des contrefaçons en provenance d'Asie rendent difficile le contrôle de la qualité. Une autre organisation, l'Inspection générale des affaires sociales, n'observe pas la présence de problèmes majeurs dans la chaîne de production, mais des carences sont notées quant à la qualité des produits actifs et des excipients en provenance de l'étranger, tandis que la validité des études de bioéquivalence et de pharmacovigilance est soulevée. Les chercheurs remettent aussi en question les méthodes d'évaluation de la bioéquivalence qui ne seraient pas sûres, soulignant que les caractéristiques pharmacocinétiques, à cause du type d'excipients et des techniques de production, différeraient entre les génériques et les médicaments de marque. Des écarts dans l'efficacité, la tolérabilité et la biodisponibilité ont été constatés pour certains médicaments. De nouvelles mesures d'évaluation seraient à utiliser pour tenir compte des variations entre les individus et les populations. À l'inverse, la Mutualité française critique des prises de position influencées par les grandes industries pharmaceutiques, leur opposant des arguments basés sur des données probantes. Elle montre, à partir d'une recension des études de la FDA (l'agence américaine de contrôle du médicament), que, dans la très grande majorité des cas, des écarts minimes dans la biodisponibilité entre les génériques pris par voie orale et les médicaments de marque, eux-mêmes sujets à des variations, confirment ainsi la validité des mesures de bioéquivalence. De ce fait, les critiques quant à la qualité, l'efficacité ou la sécurité seraient à rejeter. Ces conclusions sont partagées par les instances américaines qui confirment l'intérêt des génériques dans le traitement.

Si leurs avantages économiques sont soulignés, le statut des génériques reste donc encore problématique et des réserves sont émises par les groupes professionnels et les patients concernés quant à leur qualité et leur efficacité, même si elles varient selon les pays et les contextes culturels. Les principes et les méthodes d'évaluation des génériques font l'objet de questionnements quant à leur validité, tandis que la mondialisation pose le problème de la qualité des matériaux utilisés et de la sécurisation du processus de production. Les génériques ne font donc pas l'unanimité, 
démontrant ainsi les difficultés à établir des preuves scientifiques irréfutables quant à leur efficacité et leur sécurité, et ce, dans un domaine pourtant crucial pour la santé des populations.

\section{Pour aller plus loin}

Mutualité FRANÇAise (2012). Rapport 2012 sur les médicaments génériques. http:// www.medicamentsgeneriques.info/wp-content/uploads/2009/12/2012_12_ Médicaments-génériques.pdf

Sarradon-Eck, A., Blanc, M.-A. et Faure, A. (2007). Des usagers sceptiques face aux médicaments génériques. Une approche anthropologique. Revue d'Épidémiologie et de Santé Publique, 55: 179-185.

ToKLU, H.Z. et al. (2012). Knowledge and attitudes of the pharmacists, prescribers and patients towards generic drug use in Istanbul - Turkey. Pharmacy Practice (Internet), 10(4): 199-206. 
Pour lutter contre

la transmission du VIH, il suffit d'utiliser le condom

\author{
Jacky Ndjepel, Georges Batona, Aïssatou Coulibaly, \\ Sophie Dupéré et Laurence Guillaumie
}

Malgré les campagnes d'information et les progrès indéniables de la science, l'imaginaire collectif reste très influencé par des idées fausses sur le VIH/sida, notamment sur les modes de transmission et les moyens de prévention, comme l'utilisation du préservatif.

Les modes de transmission du VIH/sida demeurent encore flous pour bon nombre de personnes. Plusieurs études ont permis de mesurer la présence de certaines croyances à contre-courant des connaissances scientifiques actuelles au sein de la population. À titre d'exemple, une étude conduite en France en 2010 indique que 21\% des personnes interrogées, en majorité des jeunes, pensent que le virus peut se transmettre par une piqûre de moustique, $13 \%$ dans les toilettes publiques et $6 \%$ en buvant dans le verre d'une personne porteuse du virus (ANRS, 2010). D’autres idées suggèrent que l'on peut contracter le VIH en se prêtant ou en échangeant des vêtements, en ayant des contacts directs avec des personnes séropositives (embrassades, caresses, baisers, partage de repas, etc.) et avec certains animaux (singes, chats, chiens et autres). Aussi, un nombre important de personnes pense que le virus peut se transmettre par des postillons ou des crachats.

Dès son apparition en Afrique subsaharienne, le sida fut considéré par l'opinion publique comme une maladie propagée par les Blancs, pour 
décimer les populations noires, ou encore comme un virus qui se serait échappé des laboratoires des Blancs. Plus tard, avec la reconnaissance des risques associés aux rapports sexuels non protégés, l'imaginaire populaire a basculé vers une nouvelle représentation, celle d'une malédiction divine. Ainsi, les personnes contaminées par le virus seraient punies pour avoir mené une vie de débauche. De ce fait, il n'était pas rare d'associer le sida exclusivement aux prostituées et aux personnes homosexuelles. Aujourd'hui, même si les croyances africaines rejoignent celles partagées en Occident, les idées du complot blanc ou de la colère divine circulent toujours.

\section{La transmission : seulement par la voie vaginale?}

Le sida est reconnu comme une infection transmissible sexuellement et par le sang (ITSS). Il est généralement admis qu'avoir des relations sexuelles non protégées par le condom est la manière la plus courante de contracter une ITSS et, par ricochet, le VIH. Dans l'imaginaire populaire, une relation sexuelle protégée fait presque uniquement référence au port du préservatif lors de la pénétration vaginale. Cette idée est souvent renforcée et explicite dans les campagnes de sensibilisation où l'on demande habituellement à l'homme d'enfiler un préservatif au moment de la pénétration en référence au vagin ou à l'anus. Pourtant, les contacts bouche-sexe ou bouche-anus sans préservatif comportent aussi des risques de transmission du VIH, même s'ils sont jugés beaucoup plus faibles. Les simples contacts avec les organes génitaux offrent également un terrain propice pour attraper une ITSS. En effet, on compte parmi les liquides contaminants les sécrétions vaginales qui, en l'absence de pénétration, peuvent être produites par des frottements au niveau des organes génitaux. Chez les hommes, le sperme et le liquide séminal qui s'écoule au début de l'érection sont autant de liquides corporels susceptibles d'être contaminants.

Les scientifiques s'accordent pour dire que le sida se transmet de trois manières:

- La transmission par voie sexuelle, qui consiste à avoir des rapports sexuels non protégés avec pénétration vaginale, anale ou buccale avec une personne infectée. C'est le mode de contamination le plus important: $80 \%$ à $85 \%$ des personnes infectées contractent le virus 
de cette manière. Bien que la pénétration représente un risque plus grand de contracter le VIH, il en existe d'autres, même s'ils sont jugés plus faibles: de simples contacts génitaux sans pénétration produisant des sécrétions vaginales ou anales.

- La transmission par voie sanguine. Celle-ci se traduit par des échanges sanguins dans des situations bien précises, à savoir l'utilisation d'objets coupants souillés (couteaux d'excision, de circoncision, lames de scarification, de rasage, aiguilles de tatouage, seringues d'injection, piqûres accidentelles chez des professionnels de la santé) et lors de transfusions sanguines.

- La transmission mère-enfant pendant la grossesse, l'accouchement ou l'allaitement. Ce mode de contamination dépend en grande partie du niveau d'infection de la mère. Les risques de transmission sont plus élevés vers la fin de la grossesse (huitième, neuvième mois) et dépendent du type d'accouchement. Des études récentes ont démontré qu'une bonne prise en charge des femmes vivant avec le VIH leur permettait de mener à bien une grossesse et de donner naissance à des enfants en bonne santé.

Le sida est une infection qui affaiblit tout le système immunitaire d'un individu. Ce terme désigne l'ensemble des maladies qui surviennent à la suite de l'affaiblissement de l'organisme causé par le VIH. Dans les campagnes de sensibilisation, il est toujours recommandé d'adopter des comportements responsables, par exemple l'abstinence, la fidélité, la connaissance de son statut sérologique et de celui de son partenaire, l'utilisation systématique du préservatif chez les personnes actives et la stérilisation des objets contaminés. Ces recommandations constituent de bons moyens pour éviter de contracter le virus. Évidemment, le port du préservatif constitue le principal mode de prévention. En fait, si le condom est bien utilisé, le risque d'attraper le VIH est très minime. Une bonne utilisation consiste à l'enfiler avant tout contact avec le vagin, la bouche ou l'anus du partenaire.

\section{La prévention, l'arme la plus précieuse}

Les idées reçues sur le VIH/sida ont la peau dure. Bien que la maladie soit étudiée et que l'information soit mise à la disposition du public, des 
croyances fausses sont encore très ancrées dans l'imaginaire collectif. Aussi, certains chercheurs soulignent que des facteurs autres pourraient être à l'origine du sida. Dans ces conditions, on peut se demander si les mythes autour de cette grave infection finiront par tomber un jour. Toutefois, au-delà des controverses scientifiques, l'urgence d'agir et d'éduquer sur ses modes de transmission ne fait aucun doute. La prévention demeure l'arme la plus précieuse dans le combat contre le VIH/sida, en attendant d'autres avancées médicales pour éradiquer ce fléau.

\section{Pour aller plus loin}

HÉdon, C. (2008). Le sida aujourd'hui. Milan: Éditions Milan, Les Essentiels, Milan $\mathrm{n}^{\circ} 294$.

SociétÉ CANADIENNE DU SIDA (2005). La transmission du VIH: guide d'évaluation $d u$ risque. $5^{\mathrm{e}}$ éd. Société canadienne du sida, Ottawa, Canada. 


\title{
Les populations africaines ne suivent pas les consignes posologiques des traitements antirétroviraux
}

\author{
Amara Bamba, Elhadji Mamadou Mbaye \\ et Joseph Josy Lévy
}

C'est au début des années 1980 que l'épidémie du VIH/sida fait son apparition, touchant des populations de plus en plus larges, en particulier en Afrique, où près de 23,5 millions de personnes vivent avec le virus. Si, pendant plus d'une décennie, après la découverte de l'infection, les traitements antirétroviraux étaient peu développés, cette situation s'est radicalement transformée après 1996. En effet, à la suite des innovations thérapeutiques, de nouveaux antirétroviraux (ARV) ont été mis sur le marché. Prescrites en association, ces thérapies ARV constituent aujourd'hui la norme internationale du traitement. Elles ont notamment pour effet de réduire significativement la multiplication du virus, contribuant ainsi à un rétablissement des défenses de l'organisme en bloquant la progression du VIH vers le stade du sida. À cette étape, elles permettent, à long terme, un renversement de la situation avec un retour à un stade asymptomatique à long terme. Ces avantages ont eu pour effet d'augmenter l'espérance de vie et de réduire le nombre de malades et de décès dus au sida dans les hôpitaux, d'améliorer la qualité de vie, de réduire la transmission entre partenaires et à l'enfant. Le VIH/sida devient ainsi de plus en plus une maladie chronique.

Ces traitements impliquent les contraintes d'une observance thérapeutique élevée afin d'empêcher les résistances virales et la transmission 
de virus résistants aux ARV. De ce fait, on recommande un niveau d'observance très élevé, de l'ordre de $95 \%$ pour assurer une suppression du maximum de virus. Compte tenu des difficultés d'assurer une adhésion optimale, la prescription des ARV à toutes les personnes vivant avec le $\mathrm{VIH} /$ sida a suscité de nombreux débats et des prises de position critiques de hauts responsables de l'aide internationale dans le monde de la santé comme ceux de l'USAID (coopération états-unienne), de la Banque mondiale et d'autres, démontrant des préjugés tenaces à l'égard de populations vulnérables (PVVIH) et vivant dans des conditions socioéconomiques et de santé très difficiles. Ils ont affirmé que le soutien financier pour assurer l'accès des ARV aux PVVIH des pays pauvres africains serait un gaspillage de l'argent public pour plusieurs raisons. Les structures de santé locales ne seraient pas capables d'administrer le programme d'accès à cause du manque de professionnels de la santé et de la faiblesse des infrastructures, mais seraient aussi en cause des pratiques culturelles liées en particulier aux difficultés liées à la perception et à la gestion du temps, une dimension essentielle dans l'observance du traitement. Les conceptions du temps différeraient considérablement de celles qui sont prédominantes en Occident, le temps étant évalué par les rythmes naturels plutôt que selon un horaire basé sur sa mesure objective.

Ces préjugés ont été contredits par un ensemble de recherches menées dans plusieurs pays africains, qui ont démontré que le niveau d'observance des traitements antirétroviraux par les PVVIH pouvait être égal ou supérieur à celui des patients occidentaux. En Afrique du Sud, les taux d'observance des PVVIH d'origine européenne étaient comparables à ceux des patients parlant une langue nationale (Xhosa). Ces tendances se retrouvent au Sénégal, en Côte d'Ivoire et au Mozambique. Malgré des efforts à réaliser sur le plan de l'accès aux ARV en Afrique, 70 \% des personnes bénéficiant de programmes de thérapie antirétrovirale dans les dispensaires en Afrique subsaharienne continuaient de l'observer deux ans après le début du traitement. Le renforcement des ressources des organisations non gouvernementales locales mais aussi des acteurs médicaux de lutte contre le sida, l'amélioration de la prise en charge psychosociale, la lutte contre la stigmatisation, les inégalités sociales de santé et contre les discriminations ont largement contribué à l'amélioration de l'observance.

Ce haut niveau d'observance est confirmé en Amérique du Nord et dans la région subsaharienne. L'analyse des études américaines indique 
que $55 \%$ des PVVIH atteignaient les niveaux satisfaisants, alors que ce pourcentage était de $77 \%$ dans le cas des patients africains.

Ces données remettent en question les conclusions des opposants à la diffusion des ARV en Afrique. Le simplisme de leurs arguments indique qu'ils ne tiennent pas compte des contraintes complexes qui interviennent dans l'observance de ces traitements, que celles-ci soient personnelles ou plus structurelles (enjeux politiques et économiques, système de soins, rapports entre médecins et patients).

Aux contraintes financières viennent s'ajouter des facteurs qui échappent au contrôle des patients et qui affectent l'observance, comme les problèmes de disponibilité des stocks de médicaments, les problèmes de distribution ou le boycott des génériques. Les événements naturels (inondations), politiques et sociaux (grèves) survenant dans les pays peuvent aussi entraîner des interruptions non voulues dans le traitement $\mathrm{ARV}$, ce qui exige une planification de toutes les instances responsables pour assurer la continuité de la chaîne thérapeutique et les conditions de l'observance liées au minimum à l'accès aux médicaments.

\section{Pour aller plus loin}

Mills, E.J., Nachega, J.B., Buchan, I., Orbinski, J. et al. (2006). Adherence to antiretroviral therapy in Sub-Saharan Africa and North America, a meta-analysis. JAMA, 296(6): 679-69o.

Prescrire (2004). Observance des traitements en Afrique: l'obstacle est économique. Éditorial, La revue Prescrire, 24, 250 (mai) : 379-38o. http://www.prescrire.org/ editoriaux/EDI23659.pdf

Veenstra, N., Whiteside, A., Lalloo, D. et Gibbs, A. (2010). Unplanned antiretroviral treatment interruptions in southern Africa: how should we be managing these? Globalization and Health, 6: 4. http://www.globalizationandhealth.com/ content/pdf/1744-8603-6-4.pdf. 


\title{
Les méthodes contraceptives rendent stérile
}

\author{
Ramatou Ouédraogo
}

Le contrôle de la fertilité a toujours été un enjeu dans la vie des sociétés. Depuis l'Antiquité, infanticide, avortement et contraception furent pratiqués, démontrant la volonté des humains d'échapper à la fatalité. Les méthodes utilisées pour contrôler la fécondité ont connu une évolution dans le temps. Ainsi, aux méthodes dites naturelles (coït interrompu ou abstinence périodique) succèderont, au $\mathrm{xx}^{\mathrm{e}}$ siècle, les méthodes modernes constituées de dispositifs mécaniques (stérilet) ou médicaux (pilule, implant, injection, par exemple). Les méthodes médicales sont constituées de substances à activité hormonale agissant sur le fonctionnement des organes reproducteurs. Le principe est d'utiliser ces méthodes durant les périodes où la femme ou le couple ne désire pas procréer, et d'en arrêter l'utilisation une fois que le désir d'enfant se manifeste. Cependant, considérés comme des produits chimiques, bon nombre d'effets secondaires sont reprochés à ces contraceptifs, parmi lesquels le risque de stérilité. Introduites dans les pays à faible revenu à la faveur de programmes visant à maîtriser la croissance démographique des populations, et objet d'investissements divers (de la part des gouvernements, d'institutions internationales et d'ONG), les méthodes contraceptives rencontrent de nombreux obstacles. L'un d'eux est la croyance relative aux effets sur la fertilité, notamment le risque de stérilité. 


\section{Une préoccupation prégnante}

L'idée reçue autour des effets stérilisants des méthodes contraceptives est encore très présente dans les populations. Elle est souvent revenue au cours de mes enquêtes sur l'avortement auprès de jeunes femmes à Ouagadougou. Les grossesses ayant conduit la plupart de ces filles à l'avortement avaient été contractées à cause de l'absence d'utilisation de contraception malgré une absence de désir d'enfant. Aussi, après les expériences d'avortement avec complications, certaines jeunes femmes hésitaient toujours à accepter les méthodes contraceptives qui leur étaient proposées, ou les acceptaient, mais choisissaient de ne pas les utiliser une fois chez elles. Les entretiens avec ces jeunes femmes célibataires sur les motifs de leur refus et réticences ont révélé la crainte des effets secondaires, notamment le risque de stérilité. L'une d'elles, après trois avortements, pensait toujours ceci: «On dit que ce n'est pas bon, que ça rend stérile. Il paraît que ça s'entasse dans le ventre et ça finit par boucher l'utérus, et après on ne peut plus avoir d'enfant. » Des trompes à l'utérus, les substances contenues dans les contraceptifs comme la pilule, les implants ou les injectables s'attaqueraient, selon les perceptions des femmes rencontrées à Ouagadougou (Burkina Faso), aux organes reproducteurs de la femme pour, à terme, créer une stérilité. L'origine de cette croyance paraît difficile à déterminer. Il s'agit d'une forme de rumeur de par la manière dont elle s'énonce: "on dit que», «il paraît que». D’aucuns pensent tout de même que cette idée viendrait des fervents opposants à la contraception, parmi lesquels on compte les leaders religieux. Une des jeunes femmes avec qui j'ai discuté dit, en effet, avoir reçu l'information d'une amie qui l'aurait entendue, quant à elle, pendant un prêche dans son église.

Cette croyance n'est pas propre au contexte du Burkina Faso. Au cours d'un séjour au Bénin en 2013, j'ai entendu une étudiante dire lors d'une discussion de groupe sur l'avortement que «la pilule bouche les trompes, et ça fait que les ovaires et les spermatozoïdes ne peuvent plus se rencontrer». Le constat a aussi été fait en France par les enquêtes de 2007 de l'Institut national de prévention et d'éducation pour la santé (INPES), au Nigeria auprès de jeunes femmes vivant dans un camp de réfugiés et également au Québec dans une étude auprès de jeunes.

Contrairement aux femmes mariées ayant déjà entamé leur parcours génésique et qui cherchent, à travers la contraception, à espacer ou arrêter 
leur procréation, devenir stérile pour une jeune femme célibataire aspirant à la maternité revêt un tout autre sens. Chez les jeunes femmes qui n'ont pas encore entamé leur parcours génésique, le recours à la contraception vise à différer l'entrée dans la parentalité le temps pour elles de réunir les conditions propices (être mariée, se fiancer, trouver un emploi, finir ses études, etc.). Il s'agit donc, pour elles, de différer, sans la compromettre, la maternité, compte tenu de sa place fondamentale dans la construction de leur identité et dans la conjugalité. Or, les contraceptifs modernes compromettraient cette possibilité d'accéder à la maternité en temps voulu. Ces perceptions vont induire par conséquent des pratiques diverses chez les jeunes femmes, allant du refus à l'utilisation sporadique des méthodes contraceptives.

\section{Contraception et stérilité : des preuves scientifiques}

La contraception est définie par l'Organisation mondiale de la santé comme l'utilisation d'agents, de dispositifs, de méthodes ou de procédures pour diminuer la probabilité de conception ou l'éviter. Dans le cas des contraceptifs oraux, par exemple, appelés "pilules», il s'agit de comprimés d'hormones (œstrogène ou un progestatif, en fonction du type de pilule), dont le mécanisme consiste à empêcher l'ovulation ou à épaissir la glaire cervicale pour empêcher la rencontre du spermatozoïde et de l'ovule. Les effets de ces contraceptifs sont censés s'estomper une fois que la femme cesse leur utilisation, et elle est donc à nouveau féconde. Différentes recherches ont été menées sur ce retour à la fertilité après l'arrêt de méthodes de contraception. Qu'il s'agisse de recherches sur des utilisatrices de contraceptifs oraux, d'injectables ou d'implants, elles sont toutes parvenues à la conclusion d'une possible altération temporaire de la fécondité dans les premiers mois suivant l'arrêt des méthodes. Mais cela n'est jamais irréversible. Certaines femmes retrouveraient immédiatement leur fécondité, tandis que pour d'autres, un temps d'attente est nécessaire (environ trois cycles après l'arrêt de la contraception, le temps pour l'organisme de retrouver son mécanisme normal). Mais à plus de $99 \%$, elles retrouveraient leur fertilité.

L’idée reçue concernant le lien entre contraception et stérilité est une réalité. Elle contribue, autant que d'autres facteurs, au faible taux de prévalence contraceptive dans les pays à faible revenu. Son origine peut être 
recherchée, entre autres, dans la manière dont les méthodes contraceptives modernes ont été introduites et présentées aux populations. Contrairement aux pays occidentaux dans lesquels l'avènement de ces contraceptifs fait partie des luttes pour une libéralisation et un accès à ces méthodes, dans les pays en développement, notamment africains, elles ont été introduites à la faveur de politiques de population promues par des organismes comme l'OMS, la Banque mondiale, le FNUAP, etc., visant à réduire la croissance démographique. Dans un contexte où les pays en développement sont accusés de "faire trop d'enfants», avec la conscience d'une certaine volonté de l'Occident d'avoir une mainmise sur la fécondité des pays pauvres, on assiste à la naissance d'une rumeur (croyance) selon laquelle on serait prêt à tout pour parvenir à ses fins, quitte à passer par des méthodes contraceptives pour rendre stériles les femmes et les hommes. Certains évoquent même des préservatifs contenant des produits pour rendre inféconds les hommes et des pilules qui rendraient infécondes les femmes. Cependant, cette croyance, malgré sa forte présence au sein des populations, fait l'objet d'une faible attention. Dans un contexte de faible taux de recours aux méthodes contraceptives, malgré des politiques visant à lever les barrières diverses, ces idées reçues devraient porter le label "problème de santé publique» et faire l'objet d'un investissement, au même titre que celui accordé aux questions de coûts ou de barrières géographiques. Ceci est essentiel, car des femmes qui ne désirent pas nécessairement d'enfant et qui réunissent les conditions d'accès à ces méthodes les refusent et sont susceptibles d'affronter des grossesses non voulues et les dangers, pour elles et leurs enfants, de l'avortement à risque.

\section{Pour aller plus loin}

Bajos, N. et al. (2013). Tensions normatives et rapport des femmes à la contraception dans 4 pays africains. Population, vol. 68(1):17-39.

Barnhart, K., Mirkin, S., Grubb, G. et Constantine, G. (2009). Return to fertility after cessation of a continuous oral contraceptive. Fertility and Sterility, 91(5): 1654-1656.

Okanlawon, K., Reeves, M. et Agbaje, O. F. (2011). Contraceptive use: knowledge, perceptions and attitudes of refugee youths in Oru refugee camp, Nigeria. African journal of reproductive health, 14(4). 


\title{
Le nombre d'avortements provoqués augmente quand on libéralise cette pratique
}

\author{
Clémentine Rossier
}

Les avortements provoqués ou interruptions volontaires de grossesse (qui ne sont pas des interruptions spontanées ou fausses couches) sont des événements fréquents. En compilant les statistiques disponibles dans les pays où cette pratique est légale et des estimations dans les pays où elle ne l'est pas, l'Organisation mondiale de la santé estimait qu'il y a eu 44 millions d'avortements provoqués dans le monde en 2008. Près de la moitié ont lieu dans le cadre de la légalité, alors que l'autre moitié reste clandestine. La presque totalité des avortements illégaux sont pratiqués dans les pays à faible revenu. En effet, alors que les avortements sont presque tous légaux dans les pays du Nord, ce n'était le cas que de la moitié des interruptions volontaires de grossesse dans les pays à faible revenu en 2008.

\section{Légaliser pour améliorer la sécurité}

Les avortements pratiqués dans des conditions légales offrent un très grand degré de sécurité pour les femmes. Au contraire, les avortements illégaux, pratiqués dans de mauvaises conditions d'hygiène, par des personnes au degré de compétence insuffisant, avec des techniques peu sûres et à des âges trop avancés pour les femmes, peuvent conduire à de 
multiples complications. Les complications principales de l'avortement provoqué non sécurisé sont l'hémorragie, la septicémie, la péritonite et les traumatismes des organes reproductifs et abdominaux. Ces complications peuvent entraîner l'invalidité et la mort. On estimait en 2008 qu'environ 2,2 avortements provoqués sur 1000 se terminent par un décès dans les pays à faible revenu, ce qui correspond à 1 décès maternel sur 8 .

Il faut aujourd'hui relativiser quelque peu cette adéquation entre illégalité et insécurité, en raison du développement des méthodes médicamenteuses utilisées pour les interruptions de grossesse. En Amérique latine, on a observé au cours des dernières décennies une baisse du nombre de décès liés aux avortements clandestins et une diminution de la gravité des complications enregistrées dans les hôpitaux. Pourtant, la fréquence du phénomène restait inchangée et la pratique demeurait fortement criminalisée. Il semble que la diffusion du Misoprostol, une molécule suffisante pour provoquer des interruptions de grossesse et vendue couramment dans les pharmacies, en conjonction avec des efforts de diffusion de la posologie adéquate par des organisations militantes, soit à l'origine de cette diminution de la létalité des avortements. Cependant, ces pays seraient en passe de durcir l'accès à cette méthode d'auto-avortement qui met à mal leur législation.

La légalisation reste aujourd'hui encore la voie privilégiée pour améliorer durablement les conditions sanitaires des avortements et en diminuer la mortalité. La libéralisation de cette pratique rencontre toutefois des obstacles importants. Presque toutes les sociétés s'y opposent pour des raisons morales, éthiques ou religieuses. Il y a quelques décennies, les pays riches ont libéralisé leur loi sur l'avortement en dépit d'une forte opposition populaire. Les pays à faible revenu rencontrent aujourd'hui les mêmes obstacles. Un facteur clé de l'opposition des acteurs étatiques à l'avortement provoqué semble être la contradiction posée par cet acte par rapport au rôle maternel assigné à la femme. Un autre élément important tient au refus de l'idée que l'individu puisse être au centre des décisions qui le concernent. L'avortement reste ainsi couramment stigmatisé, même après sa libéralisation. Les politiques de planification familiale dans les pays où l'avortement est largement autorisé visent à en limiter le recours.

Dans les pays où la pratique n'est pas encore libéralisée, cette opposition s'exprime souvent par la crainte qu'une relaxation de la loi entraîne 
la multiplication du recours à l'avortement. Or, tant l'expérience des pays qui ont libéralisé leur loi que l'examen des statistiques mondiales d'avortement montrent un effet contraire à long terme. Le nombre d'avortements reporté aux femmes en âge reproductif est plus élevé dans les pays en développement que dans les pays développés. En d'autres termes, les régions du monde caractérisées par une part plus grande de femmes qui vivent dans des pays où l'avortement provoqué est illégal sont également les régions où la fréquence de cette pratique est la plus grande.

\section{Les vraies raisons de l'augmentation du nombre d'avortements}

Le nombre d'avortements dans un contexte particulier dépend de plusieurs facteurs. La légalité de l'avortement ne joue qu'un rôle limité dans les décisions d'interruption. Dans tous les pays du monde, il existe des services d'avortement clandestins qui peuvent répondre à la demande des femmes ou des couples. Par ailleurs, les femmes ou les couples qui décident d'interrompre une grossesse sont le plus souvent dans une situation difficile, qui les pousse à recourir à un prestataire ou à un produit abortif, même si ces services sont clandestins ou dangereux. La libéralisation permet avant tout d'améliorer l'égalité de l'accès à l'avortement et d'en garantir la sécurité pour tous. Toutes les femmes peuvent alors, en principe, accéder à des méthodes sûres sous réserve des conditions préconisées par la loi. Dans les contextes d'illégalité, les femmes qui ont moins de ressources éprouvent de plus grandes difficultés à s'assurer les services d'un prestataire ou à trouver un produit. Elles ne renoncent souvent pas à l'avortement, mais utilisent les méthodes les moins efficaces et les moins sûres.

Le facteur le plus important de la fréquence du recours à l'avortement provoqué est sans doute le désir d'éviter des naissances. Historiquement, dans les sociétés à forte fécondité, le recours à l'avortement était relativement bas. Seules les femmes se trouvant en situation de sexualité illégitime (comme les femmes non mariées) pouvaient être susceptibles de vouloir éviter une naissance. Avec la modernisation et le passage à l'économie industrielle, les couples ont cherché à diminuer leur descendance pour s'adapter à la baisse de la mortalité infantile et à la nécessité d'investir dans la scolarisation de leurs enfants. Les observateurs notent dans toutes les régions du monde une forte hausse du nombre d'avortements clandes- 
tins au début historique des baisses de la fécondité, au moment où les couples commencent à réduire la taille de leur famille, sans avoir encore développé des stratégies contraceptives efficaces.

Outre le désir (variable) d'éviter des naissances, l'autre facteur crucial du nombre d'avortements provoqués dans un contexte donné est l'utilisation de la contraception. La diminution des avortements observée par l'OMS entre 1995 et 2003 dans le monde, puis leur stagnation entre 2003 et 2008, peut être mise en lien avec l'évolution de la prévalence contraceptive dans les pays à faible revenu. Elle a d'abord augmenté, puis stagné. L'exemple récent des pays de l'est de l'Europe est également frappant. Dans ces pays, l'État a privilégié l'avortement comme mode de régulation des naissances et n'a pas développé la production des produits contraceptifs. Après la chute de l'empire soviétique, la contraception a été introduite et les taux d'avortement ont fortement diminué.

Ces facteurs (désir d'éviter une naissance et utilisation de la contraception) expliquent le niveau du recours à l'avortement dans un pays, bien plus que sa loi sur l'avortement. Parce que ces deux facteurs augmentent avec le niveau de développement d'un pays, et parce qu'ils ont un effet opposé sur le recours à l'avortement, une augmentation précède souvent son déclin. La libéralisation de l'avortement peut intervenir à n'importe quel moment de ce processus historique.

\section{Pour aller plus loin}

Ganatra, B. et al. (2014). From concept to measurement: operationalizing WHO's definition of unsafe abortion. Bulletin of the World Health Organization, 92(3): 155.

SEDGH, G., et al. (2012). Induced abortion: incidence and trends worldwide from 1995 to 2008 . The Lancet, 379 (9816): 625-632.

WHO (2011). Unsafe abortion: global and regional estimates of the incidence of unsafe abortion and associated mortality in 2008 (6th ed.), Genève: World Health Organization. 


\section{Les populations analphabètes ignorent l'importance des vaccins pour leurs enfants si bien qu'elles refusent de les faire vacciner}

Drissa Sia

La vaccination est reconnue comme l'une des interventions de santé publique les plus efficaces et efficientes. Elle a permis l'éradication de la variole et l'élimination de la poliomyélite dans plusieurs continents. Elle a permis de déplacer du premier rang des causes de mortalité les maladies infectieuses qui évoluaient sous forme épidémique, tuant ainsi des millions de personnes dans le monde.

Devant le constat de l'inaccessibilité des vaccins pour la majorité des enfants (seulement $5 \%$ d'entre eux y avaient accès en 1974 dans le monde), une initiative mondiale visant à fournir six vaccins aux enfants (contre la tuberculose, la poliomyélite, la diphtérie, le tétanos, la coqueluche et la rougeole) a été lancée dans les années 1980 et a permis de sauver plus de trois millions de vies chaque année.

Par la suite, d'autres initiatives, visant à accroître la couverture vaccinale dans les pays à faible revenu, ont vu le jour. Tous ces efforts déployés permettent de fournir gratuitement les six vaccins (plus celui contre la fièvre jaune dans certains pays) proposés par le programme élargi de vaccination (PEV) aux enfants avant leur premier anniversaire. Compte tenu de toutes ces initiatives, on est en droit d'attendre des couvertures vaccinales très élevées, sinon la vaccination complète de tous les enfants. 
Malheureusement, ce n'est pas le cas. Les taux de couverture en DTP-DTC3 (troisième dose de vaccin contre la diphtérie, le tétanos, la poliomyélite et la coqueluche) dans les régions OMS en sont une bonne illustration. Ces taux sont de $71 \%$ pour l'Afrique, $75 \%$ pour l'Asie du Sud-Est et de $85 \%$ en Méditerranée orientale.

Dans ces pays à faible revenu, surtout en Afrique, la majorité de la population est analphabète ou vit en milieu rural. Par exemple, le taux d'alphabétisation des adultes, en 2011, était, selon l'UNICEF, de $42 \%$ au Bénin et en Sierra Leone, de $57 \%$ au Bangladesh et de $63 \%$ en Inde. Dans ces pays, la proportion de la population vivant en milieu urbain au cours de la même période était de $28 \%, 31 \%, 39 \%$ et $45 \%$ respectivement au Bangladesh, en Inde, en Sierra Leone et au Bénin. Or, l'analphabétisme est la cause de plusieurs situations pouvant conduire à un mauvais état de santé. La réponse est toute trouvée! Dans de tels contextes, les personnels de santé chargés de la vaccination des enfants, pour justifier les faibles couvertures vaccinales, soutiennent que «les populations analphabètes ignorent l'importance des vaccins pour leurs enfants si bien qu'elles refusent de les faire vacciner». Demandez à des gestionnaires de programmes de santé, à des intellectuels dans plusieurs villes d'Afrique d'indiquer les raisons des faibles taux de couverture vaccinale, ils diront sans hésitation que l'ignorance des populations analphabètes est la principale raison et très peu d'entre eux évoqueront en plus le rôle du personnel de santé dans cette insuffisance de couverture vaccinale.

Avant de présenter les principales raisons qui expliqueraient les faibles couvertures vaccinales dans les pays à faible revenu, il convient de noter que l'éducation de certains membres de la société a des effets bénéfiques pour les autres membres non éduqués. Cet effet positif de l'éducation se manifeste aussi bien au sein des ménages que de la communauté où ceux-ci résident. Les données utilisées pour l'analyse des couvertures vaccinales proviennent d'individus qui eux-mêmes viennent des ménages qui font partie d'une communauté. Ainsi, ces informations recueillies pour analyser la vaccination des enfants concernent autant l'individu que les ménages et les communautés. Les auteurs qui ont utilisé des méthodes d'analyse ayant tenu compte de ces différents aspects et du contexte d'où proviennent ces informations ont montré que c'est la proportion de femmes éduquées dans le milieu considéré qui est un des facteurs explicatifs de la couverture vaccinale. 
Les principales raisons de faible couverture vaccinale sont nombreuses:

I. La faible couverture en services de santé/de vaccination occasionne des problèmes d'accessibilité géographique des sites de vaccination. Ainsi, cela se traduit par de longs et difficiles trajets pour y accéder et parfois par leur inaccessibilité pendant une période de l'année. Ce problème a d'ailleurs été reconnu dans la plupart des pays à faible revenu, de sorte qu'ont été introduites la stratégie avancée, pour laquelle les agents de santé se déplacent dans des sites choisis à cet effet, et la stratégie mobile, où des équipes de vaccination sont chargées de rejoindre des populations enclavées. Aussi cette faible couverture en services de santé est-elle étroitement liée à la quantité de personnel vaccinateur par rapport à la population. Il a été établi que plus la densité du personnel est élevée, meilleures sont l'offre de service et la couverture vaccinale.

II. Les pratiques du personnel de santé ont un rôle important. Le PEV exige que tout enfant qui entre en contact avec un centre de santé et qui ne présente aucune contre-indication de la vaccination reçoive toutes les doses qu'il devrait avoir avant de retourner chez lui. Ne pas profiter de cette chance constitue ce que l'on appelle une occasion de vaccination manquée. Les occasions de vaccination manquées ont un impact important sur la couverture vaccinale en général. Elles concernent environ un enfant sur trois.

La rencontre singulière entre les agents de santé et les membres de la communauté est le point de départ de la construction de la confiance que développent les utilisateurs des services de santé en leur système de santé. L'établissement de cette relation de confiance requiert, surtout en milieu rural, certaines conditions: (1) la continuité de la relation patient-soignant en dehors de la formation sanitaire, (2) un bon accueil et une compétence perçus par la population et une communication effective s'intéressant à la personne et non uniquement à son besoin et (3) l'habileté de l'agent de santé dans la communication, qui reste un des leviers de la fidélisation des usagers. Cette relation de confiance joue un rôle fondamental dans l'utilisation des services de prévention. Il convient aussi de noter que l'infantilisation des patients par le personnel de santé les éloignerait des services de santé, surtout pour la recherche de vaccination pour un enfant 
en bonne santé. Il n'est pas rare de voir des patients changer de centre de santé au profit d'un autre où ils se sentiraient mieux considérés et mieux servis.

III. Les rumeurs concernant la vaccination, notamment celle sur la stérilité qu'elle induirait, ont toujours existé. En revanche, ces rumeurs ne sont pas l'apanage des gens qui n'ont pas été à l'école. En effet, on se rappellera que trois États du nord du Nigeria avaient boycotté la vaccination contre la poliomyélite. Dans ce cas, ce sont les leaders politiques et religieux de ces États qui ont vu dans cette campagne de vaccination un moyen détourné de leur gouvernement fédéral d'appliquer sa politique de limitation des naissances. Ce boycott a eu des conséquences fâcheuses, non seulement pour le Nigeria avec l'augmentation du nombre de nouveaux cas de polio, mais aussi pour les pays voisins qui ont été contaminés par des souches venant du Nigeria. On pourrait faire un parallèle entre les rumeurs dans les pays à faible revenu et les mouvements anti-vaccin qui se sont développés dans certains pays développés dont les conséquences sont comparables, à savoir la résurgence de certaines maladies déjà contrôlées par la vaccination.

La variole a été éliminée. Le nombre de cas de paralysie liés à la poliomyélite a considérablement régressé. La fréquence et la gravité de certaines maladies comme la rougeole ont diminué. Ces succès face aux maladies évitables par la vaccination sont reconnus par les populations des pays à faible revenu aussi bien en milieu urbain que rural et sont attribués à l'efficacité de la vaccination. Cependant, l'apparition de manifestations post-immunisation (MAPI), telles que des abcès, peut entraîner une réticence des parents à poursuivre la vaccination de leur enfant. Mais une communication adéquate avec eux lors de la séance de vaccination sur ces MAPI permet de venir à bout de cette réticence, surtout s'il s'agit de manifestations mineures comme la fièvre. Des études anthropologiques ont montré que les problèmes de couverture vaccinale ne sont liés ni à une méconnaissance des populations ni à une négation de l'efficacité de la vaccination. L'affluence de la population à la recherche de vaccins lorsqu'une épidémie de méningite apparaît dans un pays est une illustration supplémentaire que les populations, même analphabètes, connaissent l'importance des vaccins pour eux-mêmes ainsi que pour leurs enfants. Il est alors important de souligner qu'avant d'attribuer la faible couverture 
vaccinale à l'ignorance des populations dites analphabètes, il conviendrait d'analyser les modalités de l'offre de vaccination qui leur est proposée.

\section{Pour aller plus loin}

Hutchins, S. S. et al. (1993). Studies of missed opportunities for immunization in developing and industrialized countries. Bulletin of the World Health Organization, 71:549-560.

Sanou, A. et Bibeau, G. (2009). Repères culturels communs et la promotion de la vaccination. In Tapiero, B., Carle, M.-É. (dir.), Maladies infectieuses: Illusion du risque zéro. Montréal: Éditions de l'Hôpital Ste-Justine, 215-237.

Sheppard, V. B. et al. (2004). Providing health care to low-income women: a matter of trust. Family Practice, 21: 484-491. 


\title{
16
}

\section{Le sucre est le seul responsable de l'épidémie mondiale de diabète de type 2}

\author{
Aïssatou Coulibaly, Georges Batona, Sophie Dupéré, \\ Laurence Guillaumie et Jacky Ndjepel
}

Le diabète est une maladie chronique de plus en plus répandue en Afrique subsaharienne. Le taux de prévalence est de $4,3 \%$, mais environ $80 \%$ des cas restent non diagnostiqués. Pour la personne qui reçoit ce diagnostic, des sentiments de culpabilité surgissent. La principale raison est qu'une association est faite entre une alimentation riche en sucre et le fait de devenir diabétique. Peu importe le continent où l'on se trouve, il n'est pas surprenant d'entendre des personnes affirmer qu'elles sont devenues diabétiques à la suite d'une mauvaise alimentation et surtout d'un abus de consommation d'aliments sucrés. En Côte d'Ivoire, lorsqu'une personne abuse du sucre dans sa boisson, on lui dira qu'elle est en train de s'acheter un diabète.

Le diabète est une maladie qui se développe à la suite d'un dysfonctionnement d'un organe qu'on appelle le pancréas. Cet organe permet au corps d'utiliser le sucre contenu dans les aliments consommés grâce à une substance qu'il produit appelée insuline. Il arrive que cet organe dysfonctionne, car il ne produit pas assez d'insuline ou que l'insuline produite ne peut pas être adéquatement utilisée par l'organisme, ou les deux. Dans ce cas, les aliments consommés sont transformés en sucre, mais ce sucre reste dans le sang au lieu d'être utilisé par l'organisme pour son fonctionnement et provoque ainsi des hyperglycémies. 
Les causes de cette défaillance du fonctionnement du pancréas sont complexes et varient selon le type de diabète. Le diabète de type 1 est une maladie auto-immune qui concerne $5 \%$ à $10 \%$ des cas et dont l'apparition serait liée à certains gènes. Il apparaît généralement chez les enfants et les adolescents et se caractérise par une déficience de production d'insuline par le pancréas. Le diabète de type 2, appelé aussi diabète de l'adulte ou diabète non insulino-dépendant, concerne $90 \%$ à $95 \%$ des cas et se présente surtout chez les personnes âgées de plus de 40 ans. Il se caractérise par une résistance à l'insuline et s'accompagne d'une relative déficience en insuline au début de la maladie.

À ce jour, les études scientifiques n'ont pas permis d'établir un lien entre la consommation de sucre et l'apparition du diabète de type 2. Une personne peut avoir consommé une grande quantité de produits sucrés dans sa vie sans développer un diabète ou au contraire ne jamais avoir eu tendance à la consommation de ces aliments et pourtant développer un diabète de type 2.

Toutefois, l'élément sur lequel se mettent d'accord les scientifiques concerne le lien entre le surplus de poids et le diabète. Un apport énergétique supérieur à ses besoins, que ce soit en raison de sa consommation de sucre ou d'autres aliments, entraîne un gain de poids, une accumulation de gras dans l'abdomen, voire l'obésité. Le surpoids et l'obésité constituent des facteurs de risque du diabète. Ils diminuent l'efficacité de l'utilisation de l'insuline par le corps, ce qui nécessite une augmentation de la production de l'insuline par le pancréas. Bien que la lumière ne soit pas entièrement faite sur les causes du diabète de type 2, les autres principaux facteurs de risque identifiés sont la prédisposition génétique et les habitudes de vie comme la sédentarité, la consommation excessive d'alcool et le tabagisme.

Une fois le diagnostic de diabète établi, la personne doit apporter des modifications à ses habitudes de vie qui concernent principalement l'alimentation, la pratique d'activité physique et la prise de médicaments. C’est autour de ces changements liés à l'alimentation que la question de la consommation du sucre va revenir sous une autre forme. Face au risque pour la santé associé à cette consommation, les personnes diabétiques, leur entourage et parfois même des professionnels de la santé vont proscrire la consommation de tous les sucres, y compris les sucres complexes, dits aussi glucides (le riz, la pomme de terre, les pâtes alimentaires, les tubercules comme l'igname, le manioc, le tarot, la patate douce, etc.). 
Pourtant, les recommandations internationales en lien avec l'alimentation des individus diabétiques insistent sur l'importance d'avoir un apport équilibré en glucides, lipides et protéines, tout comme pour les individus non diabétiques. Les aliments glucidiques à base de grains entiers (sucres complexes) comme les céréales, le riz, les pâtes alimentaires, les tubercules et les légumes féculents (tels que le petit pois, le maïs, la patate douce ou la pomme de terre) sont à inclure dans l'alimentation, tout en faisant attention à la taille des portions. En effet, les aliments glucidiques à grains entiers sont riches en fibres alimentaires, qui sont utiles pour faciliter le transit digestif. Pour aider les personnes atteintes de diabète dans leur choix d'aliments glucidiques, on leur conseille de privilégier les aliments glucidiques à index glycémique faible (la majorité des légumes, des fruits, des produits laitiers et des pâtes alimentaires), c'est-à-dire les aliments qui n'entraînent pas d'augmentation du taux de sucre dans le sang.

Contrairement aux idées reçues, les diabétiques n’ont pas besoin d'une alimentation spéciale. Une attention particulière devrait être portée sur les apports en gras, notamment en gras saturés, sur la consommation modérée de sel et d'aliments sucrés, sur une consommation importante de fruits et légumes et sur la consommation d'aliments faits de grains entiers. En somme, le diabétique doit suivre un plan d'alimentation correspondant à ses besoins et à ses préférences, sans tomber dans l'excès.

\section{Pour aller plus loin}

FÉdération internationale du diabète. (2013). Atlas du diabète (6 6 éd). Bruxelles, Belgique: Fédération internationale du diabète.

Grimaldi, A. (2009). Traité de diabétologie (2 éd.). Paris: Médecine-Sciences/ Flammarion.

Laville, M. et Nazare, J. A. (2009). Diabetes, insulin resistance and sugars. Obesity Reviews, 10 : 24-33. doi: 10.1111/j.1467-789X.2008.00562.X 


\title{
Les rapports sexuels avant une compétition nuisent à la performance sportive
}

\author{
Georges Batona, Aissatou Coulibaly, Sophie Dupéré, \\ Laurence Guillaumie et Jacky Ndjepel
}

Mohamed Ali, célèbre champion de boxe, avait la réputation de s'abstenir de toute activité sexuelle pendant plusieurs semaines avant chaque combat. Patrick Dempsey, acteur populaire dans la série américaine Grey's Anatomy et cycliste amateur, pratique lui aussi l'abstinence sexuelle afin d'avoir une meilleure concentration en entraînement et d'optimiser ses performances. En réalité, de nombreux sportifs, comme Ali et Dempsey, s'abstiennent de rapports sexuels avant toute compétition parce que l'acte sexuel engendrerait une dépense énergétique importante pouvant nuire à la performance sportive. Cette idée perdure depuis la Grèce antique, où les athlètes étaient déjà exhortés à observer l'abstinence sexuelle avant toute compétition. Elle se perpétue encore dans l'imaginaire collectif.

Pour s'assurer de l'abstinence sexuelle des sportifs, les entraîneurs et dirigeants sportifs vont jusqu'à interdire la présence des conjoints et conjointes lors des stages préparatoires ou des compétitions. En 2006, lors de la Coupe du monde de football, les joueurs de l'équipe nationale du Costa Rica ont été privés de leurs conjointes pendant tout leur voyage en Allemagne. Jusqu'à ce jour, durant le Tour de France, les conjointes ne peuvent pas accompagner les cyclistes. Ces pratiques s'inscrivent dans l'idée qu'une frustration sexuelle avant une compétition rendrait les 
sportifs plus agressifs, plus concentrés et potentiellement plus performants, alors que l'acte sexuel réduirait cette agressivité et l'envie de gagner. En Afrique, cette idée est tellement ancrée dans les croyances que si un footballeur d'ordinaire talentueux ne parvient pas à exprimer son talent lors d'un match, il pourra être accusé par ses supporters d'avoir eu des rapports sexuels avant le match.

Tous les sportifs ne sont cependant pas unanimes sur les effets négatifs des rapports sexuels avant une compétition. Il semble que les athlètes ne se privent pas de sexe lors des Jeux olympiques et, au contraire, s'y adonnent volontiers entre les compétitions. C'est ce que l'on peut en déduire, si l'on présume que les 150000 condoms distribués aux Jeux de Londres en 2012 ou les 220000 condoms distribués aux Jeux de Salt Lake City en 2002 ont été effectivement utilisés par les athlètes en marge des compétitions. Le sexe pourrait-il au contraire constituer le premier sport des sportifs?

Quelques scientifiques se sont intéressés à explorer la relation entre le sexe et la performance sportive. Tout d'abord, les hommes réaliseraient les mêmes performances sportives sur un tapis de course et démontreraient les mêmes niveaux de concentration, qu'ils aient eu ou non des relations sexuelles auparavant. Ainsi, le rapport sexuel n'aurait aucun impact, ni bon ni mauvais, sur les performances sportives et sur la concentration. De plus, un rapport sexuel dans des conditions normales, et conduisant à l'orgasme, ne brûle que 25 à 50 calories, soit l'équivalent de 20 marches d'escalier. Dans l'hypothèse d'une activité sexuelle ininterrompue pendant une heure, la dépense énergétique serait de 250 calories par heure, soit environ quatre calories par minute. Un tel effort est tout à fait surmontable pour un sportif de haut niveau et discrédite l'idée selon laquelle la dépense énergétique diminuerait les performances d'un athlète. Il a également été confirmé que le sexe atténuerait les douleurs musculaires liées aux entraînements chez les femmes et renforcerait les muscles chez les hommes. Enfin, au lieu de préserver le taux de testostérone dans le sang (cette hormone masculine qui renforce les muscles), une privation de tout rapport sexuel pour des sportifs pendant une période de trois mois le diminuerait plutôt. En plus de cela, le taux de testostérone masculine serait significativement augmenté à la suite d'une activité sexuelle. Ainsi, contrairement à l'idée reçue, l'activité sexuelle avant une compétition sportive pourrait conférer les bénéfices d’une agressivité accrue en raison de niveaux de testostérone supérieurs. 
D’autres bénéfices du rapport sexuel avant une compétition ont été identifiés. Selon Pamela Peeke, de l'American College of Sports Medicine, un rapport sexuel, tant pour les hommes que pour les femmes, permet de relâcher la tension et de s'endormir plus facilement. Le rapport sexuel avant une compétition aurait même un effet équilibrant sur le plan psychologique lorsqu'il se produit avec le partenaire habituel et ne revêt pas le caractère d'un exploit avant l'exploit. Ainsi, il n'aurait aucun effet sur la force ou l'endurance des athlètes, mais en revanche, pourrait être une distraction relaxante en cas d'anxiété avant une épreuve. Cependant, il ne faudrait pas que celui-ci soit associé à un manque de sommeil ou à une consommation d'alcool. Finalement, des experts s'accordent pour dire que les effets des rapports sexuels dépendent principalement de la façon dont l'athlète les perçoit. Pour Éric Blais, éducateur physique, «s'il [le sportif] pense que cela va avoir un effet négatif, c'est certainement ce qui va se produire, et inversement. C'est un peu comme les sportifs qui cessent de se raser pendant les finales, ou les joueurs qui veulent toujours porter leur chandail porte-bonheur avant une compétition».

Les recherches scientifiques ne permettent pas de soutenir l'idée selon laquelle l'activité sexuelle avant une compétition diminuerait la performance sportive. Cela est d'autant plus vrai que de nombreux sportifs utilisent la sexualité avant une compétition pour se relaxer et diminuer la pression. Aussi, les connaissances scientifiques actuelles illustrent que les niveaux de dépense d'énergie engendrés par un orgasme sont très bas.

\section{Pour aller plus loin}

Boone, T. (2008). Sex before athletic competition: Myth or fact. Edwin Mellen Pr, $1^{\mathrm{re}}$ édition.

Collier, F., (1992). La sexualité des sportives. Cahiers de Sexologie clinique, 18, $\mathrm{n}^{\circ} 111$ (nov.): 26.

Solomon, M. et Collier, F. (1991). Être femme et sportive. In P. Madelenat (dir), Etre femme et sportive, Paris, Doin, 1991: 208. 
LA SANTÉ MATERNELLE ET INFANTILE 



\title{
Les femmes n'aiment pas annoncer leur grossesse par pudeur ou parce que cela porte malheur
}

\author{
Fatoumata Ouattara
}

Pour comprendre les conduites des populations relevant de la maladie et de la santé, le recours à leur culture d'appartenance est récurrent. Ainsi, des relations entre des prestataires et des usagers des structures de soins seront focalisées sur la singularité culturelle des usagers pour expliquer dans quelle mesure des pratiques culturelles sont des obstacles pour l'accès et l'offre des soins de santé. Parmi les catégories de population, les conduites des femmes en général et de celles en situation de grossesse et d'accouchement en particulier font l'objet d'interprétations qui tendent à mettre en évidence la prégnance de représentations magico-religieuses ou des codes de bienséance liés à une culture. Il est ainsi communément admis que les femmes sont discrètes sur l'annonce de leur état de grossesse pour marquer une pudeur sexuelle ou bien parce qu'une telle annonce porterait malheur et entraînerait l'échec de la grossesse.

Les attentes des politiques de santé pour une meilleure santé des mères et des enfants préconisent un suivi médical de la femme qui devrait commencer dès le premier trimestre de la grossesse. Selon les recommandations de l'OMS, une grossesse bien suivie devrait compter une consultation prénatale à chaque trimestre. Mais la réalité diffère de cet idéal sanitaire. De fait, on observe, çà et là, une certaine irrégularité dans 
le recours des femmes enceintes aux structures de soins pour les consultations prénatales. Soit il se fait tardivement, soit il ne se fait pas du tout. Les justifications avancées relèvent, d'une part, d'un code de conduite culturelle (l'annonce rend impudique) et, d'autre part, d'un précepte magico-religieux (l'annonce porte malheur).

L'idée d'une pudeur renvoie aux catégories morales qui composent les normes de bienséance et de savoir-vivre. Par exemple, invariablement, dans de nombreuses sociétés africaines, ces normes se structurent autour de différentes pratiques, dont celles qui se rapportent à la pudeur. Celles-ci énoncent des manières d'être attendues de la femme en situation de grossesse. Il est convenu que la femme enceinte fasse preuve d'une certaine discrétion sur son état, jusqu'à ce qu'il soit perceptible par l'entourage. L'annonce d'un état de grossesse requiert donc une forme de retenue chez une femme. Au nom de cette discrétion, elle n'annonce pas en public qu'elle attend un enfant. En effet, même sous la pression de questionnements l'incitant à annoncer son état de grossesse, par pudeur, elle peut éviter en public de le dire explicitement. Tout se passe comme si un état de grossesse ne se dit pas, il s'observe.

Dans les structures de soins, il peut arriver que les femmes expriment de la réserve pour annoncer au soignant leur état de grossesse. Les questions insistantes d'un soignant peuvent faire sortir une femme en consultation de cet état de réserve et de mutisme. Taire son état de grossesse est une norme fortement intériorisée, car une femme qui la transgresse est identifiée comme une personne qui «ne connaît pas la honte», c'est-à-dire qu'elle ferait la démonstration en public d'un manque de savoir-vivre.

Les attitudes de discrétion féminines sur l'annonce de la grossesse ont été généralement interprétées comme des marqueurs de pudeur sexuelle. La pudeur sexuelle agissant comme un mode de régulation sociale, les femmes auraient, selon ce type d'interprétation, le rôle social de préserver la pudeur relevant des relations sexuelles. Parler explicitement de leur état de grossesse reviendrait alors à transgresser une telle norme de conduite qui fonde leur statut social.

La discrétion des femmes sur l'énonciation de leur grossesse est aussi concomitamment interprétée par le biais magico-religieux: cela porte malheur d'annoncer son état de grossesse. Dire sa grossesse risquerait de compromettre son évolution et d'entraîner son échec en suscitant la colère des génies et des sorciers. Ce type d'interprétation fait allusion aux des- 
criptions anthropomorphiques du monde des génies en cours dans plusieurs sociétés africaines. Dans celles-ci, les représentations populaires rendent compte d'un monde de génies pouvant interférer ou compromettre la procréation humaine.

La discrétion de la femme sur l'annonciation de sa grossesse s'insère moins dans une stratégie de démonstration de pudeur sexuelle ou de la crainte des génies que d'une conduite pragmatique relative à l'évolution même de la grossesse. Au Nord comme au Sud, toute situation de grossesse est marquée par la vulnérabilité physique de la femme et l'incertitude sur son issue. Cette dernière est plus sensible au cours des trois premiers mois de la grossesse. Or, c'est à cette période que le suivi médical devrait commencer.

S'il y a, dans la discrétion de la femme enceinte, la crainte des regards jaloux, une telle attitude traduit aussi une expression de reconnaissance d'humilité par rapport à l'évolution d'un processus qui, à terme, entérine un sentiment de plénitude. D’une idée reçue bâtie sur une norme culturelle, nous voilà dans le champ de l'universel. Des enquêtes intensives auprès des femmes au Burkina Faso conduites en milieu urbain et rural permettent d'étayer le caractère pragmatique des femmes sur la probabilité importante de fausse couche au cours du premier trimestre.

Une idée reçue ne fonctionne que parce qu'elle recouvre une part de vérité. Et dans ce sens, prétendre que la discrétion des femmes à parler spontanément de leur état de grossesse au cours des premiers mois (avant que cela ne soit perceptible) s'explique par une représentation culturelle ou une conduite de bienséance locale, c'est admettre que le sens se retrouve nécessairement dans des logiques communautaires. Ce biais interprétatif a déjà été démontré pour ce qui concerne l'analyse des pratiques de maladies à la seule aune des représentations sociales et culturelles.

Les questions que pose la discrétion des femmes sur l'annonce de leur grossesse ont moins à voir avec des craintes de sanction sociale ou magique qu'avec des conduites pragmatiques liées aux risques d'échec de la grossesse, et ce, en dépit des dispositions médicales, quelle que soit la situation de développement sanitaire d'un contexte géographique. 
98 - DES IDÉES REÇUES EN SANTÉ MONDIALE

\section{Pour aller plus loin}

Olivier de SARDAN, J.-P. et JAFFrÉ, Y. (1999). La construction sociale des maladies. Les entités nosologiques populaires en Afrique de l'Ouest. Paris, PUF.

Olivier de Sardan, J.-P., Moumouni, O. et Souley, A. (2000). «L'accouchement, c'est la guerre»: accoucher en milieu rural nigérien, Afrique contemporaine, $\mathrm{n}^{\circ}$ 195: 136-154.

Ouattara, F., Bationo, F. et Gruénais, M.-E. (2009). Pas de mère sans un «mari». La nécessité du mariage dans les structures de soins à Ouagadougou (Burkina Faso). Autrepart 4 (52): 81-94. 


\title{
19
}

\section{C'est parce que les femmes sont ignorantes qu'elles accouchent encore à domicile}

\author{
Sylvie Zongo
}

En matière de réduction de la mortalité maternelle en Afrique subsaharienne, un constat s'impose. Malgré les efforts politiques et sanitaires déployés à cet effet, on note une sous-utilisation des services de soins obstétricaux, voire l'absence de leur utilisation par certaines femmes pendant la grossesse et surtout à l'accouchement. Beaucoup de femmes accouchent encore en dehors des services de santé, notamment à domicile. Le phénomène s'observe aussi bien en milieu urbain qu'en milieu rural, mais il reste plus observé dans les zones rurales. Or, il apparaît que l'accès aux soins obstétricaux de qualité (dans les centres de santé) contribue à réduire sensiblement les décès maternels. Dans la recherche d'explications à ce phénomène, du point de vue des soignants, une idée souvent émise consiste à présenter les facteurs culturels, voire l'ignorance supposée des femmes, comme l'une des causes majeures de telles pratiques. Les femmes accouchent encore à domicile parce qu'elles ne connaissent pas les bienfaits d'un accouchement dans un service de santé et parce qu'elles ignorent les risques liés à la grossesse et à l'accouchement. Cette idée est largement répandue chez les soignants et se traduit parfois par de vifs reproches faits aux femmes qui se rendent au centre de santé après avoir accouché à domicile, ou encore par l'utilisation de termes infantilisants pendant les consultations prénatales. Elle convoque aussi régulièrement des pratiques 
culturelles considérées comme non conformes à la logique biomédicale. En effet, si les femmes n'accouchent pas dans les centres de santé ou ne les fréquentent pas régulièrement, c'est aussi parce que les représentations populaires liées à cette phase du cycle de vie de la femme et les pratiques qui lui sont rattachées ne favorisent pas le recours aux services de santé, où ces pratiques ne sont pas admises. Les facteurs culturels sont ainsi convoqués abusivement par les soignants et même par des chercheurs pour tenter de justifier les comportements des femmes vis-à-vis des services de santé. Cette attitude n'est pas exclusive au domaine de la santé. Qu'il s'agisse du domaine de l'éducation, du développement, du foncier, du politique, les facteurs culturels (l'attachement aux valeurs traditionnelles, aux vertus communautaires) restent les premiers éléments incriminés dans l'explication des échecs, des résistances, en somme, des comportements des populations africaines. Ainsi assiste-t-on à une culturalisation des faits sociaux. Une telle approche conduit à des réflexions fondées sur des préjugés et à occulter les réalités dans lesquelles évoluent les populations.

La culturalisation de l'accouchement à domicile a pour conséquence majeure de figer les comportements des femmes, vues comme ignorantes ou refusant de s'adapter aux changements, à la modernité. Sont passés sous silence les contextes dans lesquels intervient la gestion de la grossesse et de l'accouchement. De l'Afrique en Amérique latine, en passant par l'Asie, plusieurs facteurs expliquent le fait que les femmes accouchent encore en dehors des services de santé. Les aspects liés à la culture apparaissent, certes, parmi ceux-ci, mais ils ne sont pas les plus dominants ni les plus explicatifs. Si, dans certains contextes, comme en Europe, par exemple, ces accouchements sont des choix délibérés de femmes, en Afrique subsaharienne, ils sont le produit de conditions socioéconomiques, matérielles et structurelles défaillantes, de politiques de santé peu cohérentes. Dans sa critique du culturalisme pratique, D. Fassin (2001) a montré comment la sur-considération des facteurs culturels conduit à une lecture mitigée de la réalité sociale. En partant de l'interprétation du comportement des paysannes de l'Équateur vis-à-vis des centres de santé, il démontre que la faible utilisation des maternités par les paysannes indiennes résulte bien plus de problèmes sociaux et matériels que de problèmes culturels ou d'un quelconque rejet de la modernité. Les paysannes indiennes sont réticentes vis-à-vis des maternités, pas seulement 
à cause des difficultés qu'elles ont pour y accéder du fait de leur enclavement et de leur pauvreté, mais surtout à cause de leur expérience du mauvais accueil, du mauvais traitement et de certaines pratiques dans ces lieux. Il ne faut donc pas voir dans leur comportement l'effet d'un quelconque obstacle culturel, mais celui d'obstacles matériels et sociaux renforcés par l'image véhiculée par les services de santé et les soignants.

Des études réalisées au Burkina Faso ont également montré que les accouchements à domicile sont bien plus une contrainte qu'une préférence des femmes. Dans l'étude que nous avons menée dans la zone urbaine et périurbaine de Ouagadougou, il est apparu que les accouchements à domicile ne sont pas le seul fait des populations ni un problème purement culturel lié aux représentations populaires de l'accouchement (Zongo, 2004). Ils sont la résultante d'une combinaison de facteurs. Les plus importants sont la rapidité et la facilité du travail, l'enclavement des domiciles et des villages, le manque de transport pour rejoindre les centres de santé, le manque de moyens financiers. Il y a, aussi et surtout, le mauvais traitement de la part des soignants. Dans la zone périurbaine, outre les facteurs précités, c'est l'interprétation d'une ancienne politique de santé qui entretenait les accouchements à domicile. En effet, au lendemain de la conférence d'Alma Ata, des accoucheuses villageoises avaient été formées dans les zones rurales pour suivre les grossesses et effectuer les accouchements dans les postes de santé primaires. Ces postes de santé constituaient la base de la pyramide sanitaire qui caractérisait le système de santé burkinabè à cette époque. Ils étaient donc la première étape du recours aux soins. À la faveur des nouvelles orientations sanitaires, les postes de santé primaires ne font plus partie de la pyramide sanitaire. Le premier échelon du premier niveau de la pyramide est constitué par les centres de santé et de promotion sociale. Cependant, des accoucheuses villageoises ont continué d'être formées pour exercer dorénavant à domicile, avec la caution des soignants de l'aire de santé. Cette situation, malgré la réorganisation du système de santé, a donc été perçue par les populations comme une continuité de la stratégie des postes de santé primaires. Ainsi, pour les accouchements, au lieu de recourir à la maternité du centre de santé, les femmes se réfèrent aux accoucheuses, favorisant ainsi la persistance des accouchements à domicile.

En somme, ce n'est pas parce que les femmes ignorent les risques liés à la grossesse et à l'accouchement ni les avantages d'un accouchement 
dans un centre de santé qu'elles accouchent à domicile. Au contraire, elles sont bien renseignées sur ces risques et reconnaissent même les compétences des soignants par rapport à l'assistance non qualifiée à domicile. Mais c'est surtout en raison des contraintes économiques, sociales, matérielles et des dysfonctionnements des services de santé que la pratique persiste. Il y a aussi l'effet des incohérences des politiques de santé, par exemple, le statut et le rôle des accoucheuses villageoises dans le système de santé. La compréhension du comportement des femmes ne doit donc pas occulter cet ensemble de facteurs. Plutôt que de convoquer régulièrement l'ignorance des femmes pour expliquer leur faible utilisation des centres de santé pour l'accouchement, on devrait réfléchir à la manière de rendre ces centres accessibles aux populations, géographiquement, financièrement et surtout qualitativement.

\section{Pour aller plus loin}

Fassin, D. (2001). Culturalisme pratique de la santé publique. Critique du sens commun. In Dozon, J. P., Fassin, D. (dir.), Critique de la santé publique. Une approche anthropologique. Paris, Balland: 181-208.

Fassin, D. et Defossez, A. C. (1992). Une liaison dangereuse. Sciences sociales et santé publique dans les programmes de réduction de la mortalité maternelle en équateur. Cahier des sciences humaines "Anthropologie et santé publique», $\mathrm{n}^{\circ}$ 1, vol. 28, Paris, ORSTOM: 23-35.

SomÉ, T.D., SombiÉ, I. et MÉdA, N. (2011). Women's perceptions of homebirths in two rural medical districts in Burkina Faso: a qualitative perspective. Reproductive Health, 8:3. 


\title{
En Afrique, les femmes peuls n'accouchent pas à l'hôpital pour des raisons culturelles
}

\author{
Loubna Belaid et Valéry Ridde
}

La mortalité maternelle demeure un défi majeur de santé publique dans les pays du Sud. Actuellement, près de 800 femmes décèdent chaque jour pour des raisons liées à la grossesse et à l'accouchement. De plus, $99 \%$ des décès maternels ont lieu dans les pays du Sud, et notamment en Afrique, dans la région subsaharienne.

Une bonne partie de ces décès aurait pu être évitée si les femmes avaient un accès rapide et adéquat aux structures de santé. L’assistance médicale avant, pendant et après l'accouchement peut sauver des vies. C'est notamment durant l'accouchement que l'assistance par du personnel qualifié s'avère primordiale. En effet, les accouchements réalisés par des professionnels de la santé qualifiés pourraient prévenir $75 \%$ des décès maternels.

Au Burkina Faso, cette intervention sanitaire s'est traduite par la mise en place d'une politique nationale qui consiste à réduire les prix des accouchements et des urgences obstétricales dans les services de santé publics.

À la suite de l'application de cette politique, on a constaté une hausse de la couverture des accouchements par du personnel qualifié dans les centres de santé à l'échelle du pays. Cependant, des disparités sont observées entre les régions et entre les centres de santé. Par exemple, en 2010, 
quatre années après la mise en œuvre de cette politique, le taux d'accouchements assistés s'élevait à $35 \%$ dans la région du Sahel, alors qu'il était de $88 \%$ dans la région du Centre Sud. En outre, dans certaines formations sanitaires, la couverture des accouchements n'a pas du tout changé.

Cela a été le cas dans le district sanitaire de Djibo, situé dans la région du Sahel. Les agents de santé expliquent que la politique nationale n’a pas eu d'effet dans certains centres, car les femmes peuls (ethnie ${ }^{1}$ majoritaire dans le district) refusent d'accoucher à l'hôpital pour des raisons culturelles. Selon les agents de santé, une des raisons avancées est que les femmes peuls refuseraient de donner naissance à l'hôpital afin de ne pas exprimer publiquement leur douleur. En adoptant une telle attitude, elles exposeraient ainsi leur bravoure.

Il faut d'abord mentionner que cette valeur morale n'est pas spécifique des populations peuls, elle est partagée par de nombreuses sociétés africaines. On la retrouve d'ailleurs dans des représentations culturelles de nombreuses sociétés. En effet, par leur capacité à maîtriser les douleurs associées au travail d'accouchement, les femmes transmettraient à leur nouveau-né la faculté de mieux contrôler ses émotions et d'être mieux perçu par les membres de la communauté.

De plus, cette volonté de maîtriser ces douleurs ne semble pas entrer en contradiction avec le système de santé, puisque celui-ci l'encourage. En effet, les agents de santé incitent les femmes à ne pas exprimer verbalement leurs douleurs. Par ailleurs, celles qui réussissent sont valorisées par les agents de santé et sont élevées en modèles pour les autres.

Toujours selon leur point de vue, les femmes peuls opteraient aussi pour les accouchements à domicile pour des raisons de pudeur et de timidité. Or, s'agit-il réellement de traits culturels? Il faut d'abord rappeler que «l'accouchement est avant tout une affaire de femmes». Or, la majorité des soignants en milieu rural sont des hommes. En outre, le peu de professionnelles de santé qui sont dans les campagnes sont difficiles à maintenir en milieu rural au vu des conditions de travail, familiales et sécuritaires. Ainsi, la timidité et la pudeur n’ont pas à être rangées du côté de la culture, mais peuvent être considérées comme des inadéquations du système de santé face aux attentes et aux préférences des femmes.

1. Nous reprenons le terme utilisé par les professionnels de la santé de ce district pour différencier les différents groupes sociaux qui y sont présents. Le terme majoritaire est pris uniquement dans un sens numérique. 
Les agents de santé soutiennent aussi que les femmes peuls préfèrent retourner au village de leur mère pour donner naissance plutôt que de se rendre dans un centre de santé. Il ne s'agit pas non plus ici d'un obstacle culturel, mais tout simplement d'un souhait, parfois exprimé par les femmes, de pouvoir bénéficier d'un soutien familial au cours d'une période où elles se sentent en état de vulnérabilité. Il y a aussi le fait qu'à la maternité, les soignants n'autorisent pas les familles à entrer dans la salle d'accouchement en raison de l'espace réduit. Dans une salle d'accouchement, trois à quatre parturientes peuvent parfois être en travail en même temps.

Par ailleurs, le soutien social pour les femmes, du Nord comme du Sud, pendant l'accouchement est reconnu comme une stratégie efficace et contribue à renforcer l'expérience positive d'accouchement dans les services de santé publics.

En plus d'un système de santé qui reste encore faiblement à l'écoute des préférences des femmes, le manque d'infrastructures, de médicaments et des consommables nécessaires pour les soins de santé maternels est chronique dans les services de santé publics. À cela s’ajoutent les difficultés relationnelles et de communication entre les agents de santé et les parturientes. La qualité de l'accueil dans certains centres reste médiocre. Les femmes dénoncent l'absence, l'indisponibilité des professionnels de la santé dans les formations sanitaires, la violence verbale et le mépris à leur égard. De surcroît, d'autres déficiences liées aux services de santé viennent s'additionner. Elles concernent les barrières économiques et géographiques qui demeurent des freins persistants aux accouchements dans les centres de santé.

Malgré la réduction des coûts associés à la politique du Burkina Faso, les femmes continuent à payer pour leurs frais d'accouchement. Il ne faut pas non plus oublier les paiements informels exigés parfois par certains soignants, les coûts associés au transport, à la nourriture des accompagnateurs dans les centres de santé et aux consommables qui devraient être pourtant gratuits, comme les gants ou les produits de nettoyage.

En outre, les distances que les femmes doivent parcourir pour atteindre un centre de santé, les mauvaises conditions des routes, l'absence et l'inadéquation des transports (bicyclette, moto) dans les villages sont toujours des réalités vécues par les femmes enceintes lorsqu'elles souhaitent donner naissance dans un centre de santé. 
Donc, en examinant de plus près le fonctionnement actuel du système de santé, on constate que les arguments culturels semblent peu convaincants pour justifier l'absence d'effet de la politique nationale sur la couverture des accouchements assistés dans les centres de santé auprès des femmes peuls. Cela s'explique avant tout par le fait que le système de santé reste encore peu performant et est peu attentif aux préférences et aux attentes des femmes.

\section{Pour aller plus loin}

Bhutta, Z. A., Cabral, S., Chan, C.-W. et Keenan, W. J. (2012). Reducing maternal, newborn, and infant mortality globally: An integrated action agenda. International Journal of Gynecology \& Obstetrics, 119, Supplement 1(0): S13-S17.

Campbell, O. M. R. et Graham, W. J. (2006). Strategies for reducing maternal mortality: getting on with what works. Lancet, 368: 1284-1299.

Göttingen International Health Network (GIHN) (2013). Maternal-Child Health Interdisciplinary Aspects Within the Perspective of Global Health. Göttingen, Universitätsverlag Göttingen. 


\title{
La gratuité de la césarienne permet d'accélérer la réduction de la mortalité maternelle et néonatale en Afrique
}

\author{
Alexandre Dumont
}

Malgré les progrès significatifs enregistrés au cours des vingt dernières années dans le domaine de la santé reproductive, les mortalités maternelle et infantile restent un problème majeur de santé publique dans le monde. Ainsi, environ 358 ooo femmes meurent chaque année de complications liées à l'accouchement et 10 millions d'enfants âgés de moins de cinq ans connaissent le même sort, dont la vaste majorité dans les pays d'Afrique au Sud du Sahara (ASS). Une part importante (80 \%) des décès infantiles survient pendant la période périnatale (un peu avant l'accouchement et jusqu'à un mois suivant la naissance). Les causes de ces décès sont souvent liées à une prise en charge trop tardive des complications obstétricales.

On estime qu'une partie des décès maternels et périnatals liés aux complications du travail et de l'accouchement, tel qu'un arrêt de progression de la présentation du foetus depuis plusieurs heures, est généralement évitable par une césarienne réalisée en temps utile et dans les meilleures conditions. Malheureusement, cet objectif n'est pas toujours atteint dans les pays d'ASS, où la césarienne est souvent réalisée en urgence, dans des conditions précaires et trop tardivement. Dans ces pays, les distances trop longues entre le domicile des femmes et les services de santé, les faibles performances des centres de soins et les contraintes financières retardent 
souvent l'accès aux services chirurgicaux. Pour remédier à ces problèmes, plusieurs pays ont développé des initiatives pour réduire la barrière financière à l'accès aux soins obstétricaux en général et à la césarienne en particulier. Leur objectif est notamment d'accélérer la réduction de la mortalité maternelle et périnatale.

Les formules de réduction des barrières financières varient d'un pays à l'autre, allant de la subvention partielle du prix à la subvention totale (gratuité). Le Burkina Faso a adopté la formule de la subvention partielle à $80 \%$ pour les césariennes, une partie restant à la charge des familles. Au Sénégal, au Mali et au Niger, des politiques de gratuité de la césarienne ont été introduites à partir de 2005. Tous les frais au sein de l'hôpital liés à un accouchement par césarienne (bilan pré-opératoire, acte chirurgical et soins post-opératoires) sont pris en charge par l'État. Aucune de ces politiques n'avait prévu initialement de mesures d'accompagnement pour améliorer simultanément la qualité des soins obstétricaux, condition pourtant indispensable pour réduire efficacement la mortalité maternelle et néonatale. De plus, les dépenses liées au transport des patientes de leur domicile vers l'hôpital de référence ne sont généralement pas prises en compte dans ces programmes de subvention. Il est donc peu probable que les femmes les plus pauvres, résidant loin des formations sanitaires, puissent bénéficier de ces politiques.

Même si la mise en application de ces politiques de subvention nationale partielle ou totale a connu des difficultés, les études quantitatives ont confirmé leurs effets positifs sur la hausse de l'utilisation des services. Au Sénégal, par exemple, le taux populationnel de césarienne est passé de $2,87 \%$ en 2005 à $4,75 \%$ en 2011. Cependant, aucune étude populationnelle n’a montré que l'augmentation des taux de césarienne a été accompagnée d'une réduction de la mortalité maternelle et périnatale. Quelques rares études dans les formations sanitaires suggèrent en revanche une amélioration du pronostic de la mère et de son foetus lorsque la gratuité de la césarienne a été accompagnée par une intervention visant l’amélioration de la qualité des soins. Deux types d'interventions combinées à la gratuité de la césarienne ont été évalués.

La région de Kayes au Mali a mis en place en 2001 un système de référence-évacuation (SRE) des urgences obstétricales, basé sur trois composantes principales: (i) un dispositif de financement (caisses de solidarité); (ii) un système d'alerte et d'évacuation sanitaire (ambulance et radio); (iii) le renforcement des structures de référence pour les res- 
sources humaines (chirurgiens et sages-femmes) et matérielles (bloc opératoire, banque de sang). Le SRE a permis de réduire les décès maternels lors des urgences obstétricales de $50 \%$ en trois ans. L'efficacité du SRE est d'autant plus importante que la qualification du personnel de santé dans les formations sanitaires de première ligne (centres de santé communautaire) est élevée. La mise en œuvre en 2005 de la politique de gratuité de la césarienne a permis de renforcer cette tendance, en améliorant significativement le taux de couverture en césarienne et le pronostic de la mère et de son fotus par rapport aux années précédentes. Cependant, cet effet n'était observable que pour les femmes qui résidaient non loin des structures de santé.

Au Mali et au Sénégal, un programme d'amélioration de la qualité des soins obstétricaux et néonataux d'urgence (programme GESTA international) a été mis en œuvre en 2008 dans les hôpitaux de référence de ces pays. Ce programme a démarré trois ans après l'introduction de la gratuité de la césarienne. Il était basé sur la pratique des revues de décès maternels et la formation continue du personnel de santé en soins obstétricaux et néonataux d'urgence. Les revues de décès maternels ont pour objectif de rechercher de manière qualitative et approfondie les causes et les circonstances entourant les morts maternelles survenues dans les établissements de santé. Cette approche représente un outil essentiel pour définir les actions d'amélioration de la qualité des soins à mettre en œuvre. Deux ans après la mise en œuvre de ce programme, la mortalité maternelle hospitalière a diminué de $15 \%$ dans les hôpitaux bénéficiaires du programme par rapport aux hôpitaux non bénéficiaires. La mortalité néonatale immédiate (inférieure à $24 \mathrm{~h}$ ) a suivi la même tendance avec une diminution plus marquée de $26 \%$. L'intervention a conduit à la mise à jour ou à l'élaboration de nouvelles recommandations pour la pratique clinique, à des changements de pratiques professionnelles, telles qu'une augmentation des transfusions sanguines pour les patientes présentant une hémorragie obstétricale, une augmentation des césariennes avant le travail et une diminution des césariennes urgentes pendant le travail.

Les résultats de ces deux études suggèrent donc que la gratuité de la césarienne ne peut permettre, à elle seule, de résoudre le problème de l'accès pour toutes les femmes à des soins obstétricaux de qualité. Dans la région de Kayes, au Mali, c'est un système de référence organisé des urgences obstétricales qui a permis, dans un premier temps, d’améliorer le pronostic des mères et de leurs nouveau-nés. La gratuité de la césarienne 
a renforcé cette tendance en éliminant la barrière financière liée aux dépenses au sein de l'hôpital. Cependant, quelques années après la mise en œuvre de la politique de gratuité, on constate que la plupart des caisses de solidarité pour la prise en charge des urgences obstétricales ne fonctionnent plus, ou nettement moins bien qu'avant la gratuité. En effet, les communautés ne voient plus vraiment l'intérêt de cotiser pour ces caisses alors qu'on leur parle de gratuité des soins. Les dépenses effectuées avant de se rendre à l'hôpital représentent à nouveau une barrière financière à l'accès aux soins. Une part importante des dépenses de soins est liée au transport des femmes du domicile vers les structures de santé. Certaines dépenses dites catastrophiques font alors basculer les familles vers une situation d'extrême pauvreté, entraînant la déscolarisation des enfants et une sous-alimentation chronique. Ces résultats ont montré les limites de la politique de gratuité telle que formulée au Mali.

Lors de l'émergence des politiques de subvention totale ou partielle de la césarienne, il semblait évident que l'élimination d'une grande partie des barrières financières puisse résoudre le problème de l'accès à des soins obstétricaux essentiels et contribuer ainsi à réduire la mortalité maternelle et périnatale. Les données quantitatives confirment que ces programmes ont bien un effet positif sur l'utilisation des services, sans toutefois régler le problème de l'accessibilité géographique aux structures de santé pour certaines femmes. Sans des mesures d'accompagnement pour améliorer simultanément la qualité des soins obstétricaux, il est peu probable que ces politiques aient un impact sur la santé des mères et des nouveau-nés. Ces mesures d'accompagnement sont nécessaires pour contrecarrer les effets inattendus ou pervers de la gratuité de la césarienne en ASS liés à un risque réel de sur-utilisation des services pour les plus nantis.

\section{Pour aller plus loin}

Arsenault, C., et al. (2013). Emergency Obstetric Care in Mali: Catastrophic Spending and its Impoverishing. Bull World Health Organ, 91(3): 207-216.

Fournier, P. et al. (2014) The free caesareans policy in low-income settings: an interrupted time series analysis in Mali (2003-2012). PLoS One, 19;9(8): e105130. doi : 10.1371/journal.pone.0105130.

WANiez, P. et al. (2006) L'abus de recours à la césarienne au Brésil: dimensions géographiques d'une aberration médicale [Internet]. Available from: http://pmb. santenpdc.org/opac_css/index.php?lvl=notice_display\&id=26141 


\title{
Les mères africaines, par manque d'éducation, n'amènent pas leurs enfants au centre de santé
}

\author{
Jean-Yves Le Hesran
}

Les taux de morbidité et de mortalité infanto-juvénile sont extrêmement élevés en Afrique subsaharienne. Le risque qu'un enfant meure avant l'âge de cinq ans y est 16 fois plus grand que dans les pays à revenu élevé. Les naissances prématurées et les infections sont à l'origine de la plupart des décès néonatals. Après cette période, et pendant les cinq premières années de vie, les principales causes de mortalité sont la pneumonie, la diarrhée et le paludisme. La malnutrition est un facteur sous-jacent dans environ $45 \%$ des décès, car elle rend les enfants plus vulnérables aux maladies graves.

Plus de la moitié des décès d'enfants de moins de cinq ans est due à des maladies qui pourraient être évitées ou traitées par des interventions simples et d'un coût modéré. Des actions de promotion de la santé sont mises en place pour répondre à ces problèmes de santé publique majeurs. Les interventions qui y sont associées ciblent l'adoption de comportements sains et l'autonomisation des individus, des familles, des ménages, et notamment des mères pour leur permettre de prendre des mesures adaptées face aux problèmes de santé des enfants qu'elles ont à gérer.

Toutefois, toutes ces actions menées suggèrent également l'idée d'une responsabilité des mères africaines, qui ne sauraient pas traiter correctement 
leurs enfants et les emmener à temps au centre de santé parce qu'elles ne seraient pas assez éduquées.

Pourtant, des programmes d'éducation pour la santé sont proposés depuis de nombreuses années, sans résultats très probants. Il est donc intéressant de revoir comment, et dans quel cadre, les mères africaines prennent en charge la santé de leurs enfants.

La mère devant une fièvre chez l'un de ses enfants doit faire face à diverses contraintes et en tout premier lieu à des contraintes financières. Une large part des populations d'Afrique subsaharienne vit actuellement dans un contexte de grande pauvreté. Depuis l'initiative de Bamako, en 1987, dans une logique de recouvrement des coûts, les frais de santé doivent être, en grande majorité, réglés directement par les patients. De plus, l'insuffisance ou l'inaccessibilité d'assurances de santé publiques ou privées empêche nombre d'individus de bénéficier d'une couverture contre le risque de maladie. Sans protection sociale, les populations doivent prendre en charge le coût direct de leur santé, et ceci dans un souci constant d'économie. Aller au dispensaire signifie pour les ménages l'engagement de frais directs (prix de consultation, coût des examens et des médicaments) et de frais indirects de transport et de perte de temps de travail.

Se pose également la question de la qualité de l'accueil et du service rendu. Pour différentes raisons (manque de moyens financiers, manque de personnel), le temps de consultation dans les dispensaires est très court, il y a très peu de dialogue entre le personnel soignant et la mère. De plus, le geste médical est très souvent stéréotypé: examen clinique rapide et traitement symptomatique ou traitement test par des antipaludiques ou des antibiotiques. La consultation s'achève généralement par « revenez si cela ne passe pas».

La mère apprend rapidement cette étape de test thérapeutique qui est le plus souvent efficace, puisqu'une grande partie des fièvres de l'enfant sont résolutives spontanément et donc traitées efficacement avec un antipyrétique ou un antipaludique.

De plus, le retrait du secteur public du financement de la santé a eu d'importantes conséquences, en particulier celle de permettre le développement du secteur informel du marché du médicament. Ce marché, présent partout, dans les marchés hebdomadaires, mais également dans les quartiers par l'intermédiaire des marchands ambulants, propose l'ensemble des médicaments prescrits dans les dispensaires. 
Enfin, il faut rappeler que les fièvres de l'enfant sont fréquentes et que leur prise en charge représente des dépenses importantes et répétées pour les familles. Ainsi, pour des raisons d'économie de temps, mais aussi par ce qu'elles ont appris de leur passage dans les dispensaires, les mères font, en cas de symptomatologie bénigne et reconnue, de l'automédication raisonnée. Elles s'occupent elles-mêmes de la première phase du traitement d'une petite maladie.

On avance souvent que les parents en Afrique utilisent en première intention les traitements traditionnels. Cela est vrai et faux. Les familles utilisent effectivement fréquemment des tisanes pour être en bonne santé mais, en cas de symptômes précis (fièvre, douleur, etc.), elles ont plus souvent recours à un médicament moderne. L’utilisation des médicaments est donc une pratique courante avec pour preuve l'état florissant du marché du médicament en Afrique.

Le problème de la prise en charge de la santé des enfants réside plutôt dans le délai de réaction en cas de persistance des symptômes ou d'aggravation de l'état de santé de l'enfant. Amener un enfant au dispensaire ou à l'hôpital représente une dépense potentielle importante qui nécessite une décision consensuelle de la famille et un temps pour mobiliser de l'argent. De plus, si l'état de l'enfant est grave, sa prise en charge correcte n'est pas possible dans toutes les structures de soins, ce qui nécessite un transfert vers un centre de santé mieux équipé et donc un délai et des dépenses supplémentaires.

Ainsi, combattre la morbidité ou la mortalité chez l'enfant nécessite non seulement de travailler auprès des femmes, avec une éducation sanitaire adaptée, mais aussi une amélioration des conditions d'accueil dans les services de santé, une augmentation des capacités techniques de ces centres et, enfin et surtout, une politique de prise en charge de la santé des populations par des stratégies de gratuité ciblée (prise en charge gratuite du paludisme pour les enfants de moins de cinq ans) ou le développement de la couverture médicale universelle (CMU), dont l'objectif est de faire en sorte que tous les individus aient accès aux services de santé sans encourir de difficultés financières. 


\section{Pour aller plus loin}

Baxerres, C. et Le Hesran, J.-Y. (2006). Le marché parallèle du médicament en milieu rural au Sénégal. Les atouts d'une offre de soins populaire. Anthropologie et Sociétés, vol. 30, $\mathrm{n}^{\circ} 3:$ 219-230.

Baxerres, C. et Le Hesran, J.-Y. (2010). Quelles ressources familiales financent la santé des enfants? Les difficultés du recours aux soins pour traiter le paludisme en milieu rural sénégalais. Revue Tiers Monde, $\mathrm{n}^{\circ} 202$.

Ridde, V. (2012). L'accès aux soins de santé en Afrique de l'Ouest. Au delà des idéologies et des idées reçues. Les Presses de l'Université de Montréal. 


\title{
Les agents de santé communautaires peuvent soigner les enfants fébriles dans les régions rurales d'Afrique subsaharienne
}

\author{
Thomas Druetz
}

Au lendemain de la conférence d'Alma-Ata en 1978, des agents de santé communautaires (ASC) ont été déployés dans la plupart des pays à revenus faibles ou modérés afin de mettre en œuvre la politique des soins de santé primaires. Progressivement délaissés en Afrique subsaharienne, notamment à la suite des influences néo-libérales qui ont restreint les politiques publiques et sapé la vision holistique et positive de la santé exprimée à Alma-Ata, l'intérêt porté aux ASC y est aujourd'hui en pleine recrudescence. Jeffrey Sachs, une autorité mondiale en santé publique, a récemment appelé à former un million d'ASC sur le continent africain

Les agents de santé communautaires ont pour mission d'améliorer la santé de la population dans leur village. Ils partagent deux caractéristiques essentielles: (1) ils proviennent du village où ils opèrent et (2) ils reçoivent une formation ad hoc de courte durée, de quelques jours à quelques semaines tout au plus. Les ASC sont souvent considérés comme le prolongement du système de santé dans les villages, même si, dans la plupart des cas, ils ne font pas partie de la fonction publique, ils ne reçoivent pas de salaire officiel et leurs actes ne sont pas réglementés. 
avant le terme des Objectifs du Millénaire pour le développement, prévu en 2015. Bien que le spectre des missions qui leur échoient soit vaste, nous traiterons dans ce chapitre de deux idées reçues concernant un axe majeur de leur utilisation actuelle: la prise en charge thérapeutique des enfants fébriles.

Ce regain d'intérêt pour les ASC en Afrique pour soigner les enfants fébriles marque le passage d'une idée reçue à une autre. La première idée, qui n'est combattue que depuis récemment, consistait à voir dans toute fièvre de l'enfant un cas de paludisme. Lorsqu'un enfant fébrile venait en consultation, l'ASC lui administrait automatiquement un traitement antipaludéen s'il en disposait. Depuis l'arrivée de tests de diagnostic rapide du paludisme, cette idée est graduellement remplacée par une autre, celle d'une prise en charge combinée du paludisme et de la pneumonie par les ASC. Les ASC administreraient des antipaludéens aux enfants fébriles dont le test est positif, et des antibiotiques à ceux qui présentent un ou des signes de pneumonie. Cette stratégie permettrait la prise en charge sur le plan communautaire des deux maladies auxquelles on attribue le plus de décès infantiles en Afrique subsaharienne.

\section{Toute fièvre est causée par le paludisme}

L'administration systématique d'antipaludéens aux enfants fébriles, sans que le diagnostic ait été confirmé, a longtemps été recommandée dans les pays d'Afrique où le paludisme est très présent. Deux facteurs ont justifié cette stratégie. D'une part, il était utopique de vouloir confirmer tous les cas de paludisme en laboratoire, particulièrement dans les zones rurales. D'autre part, il était important d'administrer rapidement (moins de 24 heures après l'apparition des symptômes) un traitement aux enfants ayant le paludisme sous peine de voir leur état empirer et leur probabilité de décès augmenter. L'idée que toute fièvre est causée par le paludisme a donc été inculquée aux agents de santé et aux ASC. Elle a ensuite interagi avec les contextes locaux, où des préconceptions à l'égard de la fièvre circulaient déjà dans les communautés.

L'utilisation d'ASC pour administrer des antipaludéens dans les villages peut accélérer le recours aux soins des enfants fébriles et diminuer le délai entre l'apparition de la fièvre et le traitement. L'efficacité potentielle de cette stratégie pour réduire la morbidité et la mortalité attribuables au 
paludisme a été démontrée, de même que sa faisabilité et son acceptabilité par les populations.

Par contre, les études ont aussi montré que la proportion d'enfants fébriles qui sont véritablement atteints de paludisme est extrêmement variable en fonction de l'épidémiologie locale de la maladie. Ce constat s'est empiré avec le recul du paludisme observé dans plusieurs pays africains depuis les années 1990. Le nombre élevé de faux cas présumés de paludisme a entraîné des effets néfastes. On a ainsi observé une surprescription d'antipaludéens, ce qui a encouragé les ruptures dans les chaînes d'approvisionnement ainsi que l'émergence de résistances du parasite à l'égard des molécules. Également, pour les enfants qui souffrent en réalité d'autres infections ou maladies, la présence présumée du paludisme dans toute fièvre retarde l'établissement du bon diagnostic ainsi que la prise en charge thérapeutique appropriée.

La réduction progressive du fardeau global du paludisme, l'élaboration de tests de diagnostic rapide et la crainte de nouvelles résistances ont incité, en 2010, l'OMS à recommander aux États de ne traiter désormais que les cas confirmés de paludisme. Dans ce nouveau contexte, les ASC sont progressivement formés à utiliser les tests de diagnostic rapide et à n’administrer les antipaludéens qu'aux cas positifs. Mais ce nouveau protocole de prise en charge entre souvent en conflit avec l'idée qu'on leur inculquait depuis des années et selon laquelle tout enfant ayant une fièvre souffre de paludisme. Des études récentes montrent que ce nouveau protocole se heurte à une certaine opposition de la part des agents de santé et des mères qui amènent leur enfant fébrile en consultation. Par conséquent, de nombreux antipaludéens continuent d'être administrés aux enfants fébriles dont le test est pourtant négatif.

\section{L'ASC pour traiter de façon combinée le paludisme et la pneumonie}

Au fur et à mesure que l'utilisation de tests de diagnostic rapide se répand, une nouvelle idée progresse. Les ASC administreraient des antipaludéens aux seuls cas confirmés de paludisme, et procureraient des antibiotiques aux enfants fébriles qui présentent un ou plusieurs signes de pneumonie. Plusieurs éléments justifient cette stratégie. D’une part, en Afrique subsaharienne, la pneumonie se situe au deuxième rang (après le paludisme) des plus importantes causes de mortalité - près de 750 ooo décès infantiles 
annuels lui sont attribués. D’autre part, la pneumonie, tout comme le paludisme, nécessite une prise en charge rapide sous peine d'augmenter les probabilités de décès.

Plusieurs études ont montré que les ASC peuvent efficacement prendre en charge la pneumonie infantile et réduire ainsi la mortalité qui lui est attribuée. Un protocole a été développé pour leur permettre de diagnostiquer la pneumonie chez l'enfant et de distinguer les formes sévères des formes simples. Des outils ont été conçus pour les aider à déceler un taux élevé de respiration par minute, qui est un signe clinique caractéristique de la pneumonie. Les ASC ont montré une bonne capacité à utiliser ces outils et à suivre fidèlement le protocole. Cependant, la grande majorité des études ayant démontré le succès de la prise en charge de la pneumonie par des ASC ont été menées en Asie, dans un contexte radicalement différent de celui de l'Afrique subsaharienne. En effet, outre la présence beaucoup plus marquée du paludisme, l'Afrique subsaharienne se caractérise par un accès plus restreint aux soins de santé, par des services publics plus rares et par un taux d'alphabétisation de la population et des ASC moins élevé.

En réalité, l'impact de la prise en charge de la pneumonie par des ASC en Afrique reste à démontrer. En ce qui concerne la performance des ASC, les rares études qui l'ont mesurée suggèrent que les ASC éprouvent des difficultés à suivre le protocole décisionnel, particulièrement s'ils sont formés à prendre en charge plusieurs maladies (par exemple, le paludisme et la pneumonie) ou si l'enfant présente des signes de sévérité. Les déterminants de bonne performance des ASC sont pour la plupart inconnus, de même que les critères associés à une bonne implantation de cette stratégie. Les taux de sur-prescription et de sous-prescription d'antibiotiques, le traitement administré en cas de pneumonie, varient grandement d'une étude à l'autre, ce qui est préoccupant dans une perspective de santé publique. En effet, dans l'hypothèse où cette stratégie est implantée à grande échelle, elle pourrait encourager le développement de résistances aux antibiotiques. De surcroît, l'épidémiologie de la pneumonie dans les milieux ruraux d'Afrique subsaharienne est mal connue, notamment la proportion des cas causés par des infections autres que bactériennes - et pour lesquels les antibiotiques ne sont pas indiqués.

Ainsi, le recours aux ASC peut permettre d'améliorer l'accès rapide à des traitements efficaces dans les zones rurales. Toutefois, le potentiel 
des ASC pour diminuer la mortalité des enfants fébriles a été limité par deux idées qui ont gouverné leur utilisation. Ces idées sont tombées dans le même travers: miser sur l'efficacité des traitements administrés (antipaludéens ou antibiotiques) plutôt que d'accorder une considération suffisante à la pluralité des causes de la fièvre ou aux difficultés d'implantation en Afrique subsaharienne d'une stratégie éprouvée en Asie. Le recours aux ASC sur le continent africain ne constitue donc pas une solution simple et facile au problème crucial du manque d'accès aux soins des populations rurales.

\section{Pour aller plus loin}

Baiden, F., Webster, J., Owusu-Agyei, S. et Chandramohan, D. (2011). Would rational use of antibiotics be compromised in the era of test-based management of malaria? Tropical Medicine \& International Health, 16 (2): 142-144.

Druetz, T., Siekmans, K., Goossens, S., Ridde, V. et Haddad, S. (2015). The community case management of pneumonia in Africa: A review of the evidence. Health Policy Plan. 30 (2), : 253-266.

FAYE, S. (2012). Améliorer la prise en charge du paludisme par les tests de diagnostic rapide (TDR) : appropriation par les prestataires et bénéficiaires de soins au Sénégal. Bull Soc Pathol Exot, 105(3) : 237-244. 



\section{LES POPULATIONS VULNÉRABLES}

\section{ET L'ÉQUITÉ}





\title{
Les prestataires confessionnels servent les pauvres
}

\author{
Fahdi Dkhimi et Bart Criel
}

Les hôpitaux d'inspiration religieuse ont-ils encore le monopole du cœur? Les prestataires dits confessionnels ont une place particulière dans les systèmes de santé d'Afrique subsaharienne. Ils bénéficient d'une image de marque forte, ils sont les plus appréciés par les usagers des services de santé. Au-delà de la qualité perçue des soins délivrés, une des raisons pour lesquelles ils jouissent d'une bonne réputation est leur caractère charitable. En effet, en Afrique subsaharienne, et plus particulièrement en milieu rural, l'image de l'hôpital confessionnel desservant les pauvres fait encore partie des images symboliques dominantes. Mais existe-t-il des preuves pour justifier cette réputation?

En 2002, le président de la Banque mondiale de l'époque, James Wolfensohn, déclarait: "La moitié du travail dans l'éducation et dans la santé en Afrique est réalisée par les églises... Mais elles ne se parlent pas entre elles, et ne nous parlent pas non plus.» À l'époque, il était difficile de justifier cette impression générale par des données chiffrées. Aujourd'hui encore, malgré plusieurs recherches récentes, le poids des structures confessionnelles dans l'offre de soins reste mal connu. Le plaidoyer pour une plus grande considération de ces prestataires dans les stratégies nationales se base souvent sur des données fragmentées, voire contradictoires.

Néanmoins, au cours de la dernière décennie, cette image de prestataires des pauvres conférée aux structures de santé confessionnelles a 
constamment été mise à mal par les transformations systémiques qu'ont connues ces structures de soins. L'œil averti des utilisateurs et des analystes des systèmes de santé n'aura pas manqué de constater l'inflation rapide du prix des soins chargés aux patients. Certes, les hôpitaux confessionnels continuent de jouir d'une image positive au sein des populations, mais les prix affichés posent la question de leur accessibilité financière, surtout quand on les compare aux prix appliqués par les prestataires du secteur public. Une étude récente menée dans 14 pays a constaté que non seulement les prestataires confessionnels ont tendance à être moins fréquentés par les populations pauvres que par les autres mais, en plus, que les structures confessionnelles ne servaient pas les pauvres en plus grand nombre que les structures publiques. Un tel constat contredit ainsi l'imaginaire collectif.

Qu'est-ce qui explique ce changement? La première raison est liée au redéploiement des structures publiques de soins sur les territoires nationaux. Après une période de sous-financement aiguë, les années 2000 ont été marquées par une augmentation assez importante des budgets alloués aux services de santé, grâce notamment à une croissance forte de l'aide internationale. Une partie importante de cet argent a été consacrée à la construction ou à la rénovation d'infrastructures publiques, souvent en milieu rural. Les hôpitaux confessionnels, qui avaient été installés par les missionnaires dans des zones reculées et moins privilégiés pour des raisons autant humanitaires que religieuses, y étaient jusque-là en situation de quasi-monopole. Ils se sont retrouvés en situation de concurrence avec des infrastructures publiques plus récentes, et souvent mieux fournies en personnel. Paradoxalement, même si cette situation a pesé sur la fréquentation des hôpitaux confessionnels, ces derniers n'ont pas été les plus affectés. Ce sont surtout les structures de soins de santé primaires, gérées par les institutions religieuses, qui ont connu le plus de difficultés. Nombre d'entre elles ont d'ailleurs été cédées au gouvernement ou tout simplement fermées au cours de la décennie, afin de concentrer les ressources plus restreintes sur les hôpitaux. Il y a donc eu une nette baisse de fréquentation du secteur de santé confessionnel, relativement au secteur public.

Mais la baisse de fréquentation des structures confessionnelles n'est pas uniquement liée à cette concurrence accrue, que les États ont tenté d'endiguer en établissant des partenariats public-privé avec les structures 
confessionnelles. Une autre raison majeure est liée aux orientations stratégiques que les établissements confessionnels ont prises face aux problèmes récurrents de financement auxquels ils ont fait face avec la diminution structurelle des dons en provenance de leurs partenaires conventionnels, c'est-à-dire les églises et congrégations des pays occidentaux.

Face à ces nouvelles contraintes financières, nombre de structures confessionnelles ont, en effet, été contraintes de diversifier leurs sources de revenus pour générer les fonds nécessaires à leur fonctionnement. Au cours des années 2000, les établissements d'inspiration religieuse se sont donc engagés dans des stratégies de développement de partenariats avec des réseaux plus larges de donateurs. Ils ont fait appel aux bailleurs publics mais aussi, et surtout, aux États pour endiguer cette chute de fréquentation. Néanmoins, la plupart des nouveaux subsides sollicités présentaient des conditions et imposaient des cibles spécifiques. Y accéder exigeait donc d'apprendre à gérer différents programmes de santé publique, à soumettre des rapports d'activité, à produire des états financiers pour des institutions qui, depuis leur création, fonctionnaient quasi exclusivement sur la base d'un budget annuel stable et jouissaient d'une marge de manœuvre importante. Un véritable changement de culture de gestion que les établissements confessionnels devaient intégrer, mais qui ne s'acquiert pas en un jour. De plus, ces types de financements ciblés ne permettaient pas à ces hôpitaux de couvrir l'ensemble de leurs charges. Enfin, les représentants de l'État ont souvent mal apprécié la situation financière délicate et discrète des hôpitaux confessionnels. Ils pensaient que les institutions religieuses avaient les ressources nécessaires pour ne pas être prioritaires quand il s'agissait de débourser les ressources publiques. Cela entraînait des délais de paiement, voire leur absence, qui plaçaient les prestataires de soins dans des situations délicates en matière de trésorerie.

Dans la plupart des cas, cette transition vers un nouveau mode de financement a été entamée tardivement. Face à l'urgence de la situation, beaucoup d'établissements de soins d'inspiration religieuse ne sont pas parvenus à diversifier leurs sources de financement pour couvrir l'ensemble de leurs besoins. La plupart ont donc connu une période de grandes difficultés financières. Leur seul levier pour faire face à leurs charges a été d'accroître la participation financière du patient. Cela a marqué le début d'un processus d'augmentation des tarifs des consultations dont les conséquences sont encore largement visibles aujourd'hui. 
Dans un premier temps, l'application de cette nouvelle politique tarifaire n'a été que partiellement efficace. La preuve en est la croissance exponentielle du montant des impayés dans les exercices comptables. Mais, à la différence de la période précédente, qui avait également son lot d'impayés, le recouvrement des paiements était devenu indispensable au fonctionnement des établissements. De plus, les églises, qui disposaient de la très grande majorité des établissements de soins d'inspiration religieuse, ont également exigé une plus grande autonomie financière de leurs hôpitaux, afin d'utiliser leurs ressources à d'autres fins et pour d'autres priorités du clergé africain.

Ils ont alors adopté une variété de mesures pour assurer le recouvrement des créances - visites à domicile, convocations, etc. Bien souvent, cette tâche était assurée par les assistants sociaux recrutés par l'hôpital. Ils avaient la charge de mener des enquêtes sociales auprès des familles créditrices et qui pouvaient, le cas échéant, exempter les indigents, qu'ils identifiaient sur la base de critères pas toujours constants et rigoureux. Mais le poids administratif et financier de ces méthodes de recouvrement a amené ces structures à se protéger de plus en plus contre tout risque d'impayés. Ils ont adopté des stratégies, allant jusqu'à exiger des dépôts de garantie en nature ou en argent avant d'autoriser l'admission d'un patient au sein de leur établissement.

Cette nouvelle politique tarifaire a rapidement eu un effet négatif sur la fréquentation des hôpitaux. Le nombre de patients a chuté, engendrant un différentiel croissant entre les recettes mobilisées par le paiement à l'acte et les besoins en financement. Les structures confessionnelles sont alors entrées dans un cercle vicieux. Face à l'érosion de leurs ressources propres et à leur incapacité de couvrir leurs frais fixes de fonctionnement, elles ont continué à augmenter leurs tarifs. Or, plus les prix chargés aux patients ont augmenté, plus ces structures se sont vidées. De plus, cette chute de fréquentation a entraîné une recomposition de la typologie des patients. Les catégories socioéconomiques défavorisées sont celles qui ont le plus déserté ces établissements de soins. Certes, il reste encore quelques modalités pour aider les plus indigents, mais la grande majorité de pauvres n'a tout simplement plus accès à ces hôpitaux.

Aujourd'hui, il n'est pas rare de trouver des hôpitaux confessionnels dont les niveaux d'utilisation sont faibles, avec par exemple des taux d'occupation des lits de $50 \%$ à $60 \%$. Cela pose de sérieuses questions sur 
l'efficience de ces structures. Certaines ont décidé d'investir dans l'aspect qualité au détriment de l'accessibilité en offrant des services adaptés à une population solvable du milieu rural (chambres individuelles, par exemple). D’autres délivrent des soins spécialisés, indisponibles dans les structures publiques avoisinantes, mais qui restent relativement chers. C'est du moins ce que l'on constate en Afrique de l'Est.

Les hôpitaux confessionnels ont donc progressivement changé de profil au cours des dernières années, au détriment de leur image de structures des pauvres. C'est en tout cas l'impression générale partagée par nombre d'usagers d'Afrique subsaharienne. Au cours des dernières années, grâce à l'inclusion des structures confessionnelles dans les programmes verticaux (par exemple pour la vaccination, la santé de la reproduction, la prise en charge des personnes vivant avec le VIH/sida), ces structures d'inspiration religieuse ont rouvert leurs portes à un public plus large. Mais la tendance à la privatisation croissante de l'offre de soins confessionnelle - choix par défaut - a pénétré les esprits. Les défenseurs les plus ardents sur les scènes nationales du paiement à l'acte se trouvent aujourd'hui dans les rangs des institutions religieuses.

Peut-on en conclure que ces structures confessionnelles ne sont plus pour les pauvres? Le problème, encore une fois, est lié au manque d'information. La plus grande partie de ce que nous savons reste basée sur des anecdotes, ou sur des collectes de données ad hoc, alors qu'il existe une diversité considérable sur le continent de la situation des hôpitaux confessionnels. Néanmoins, même s'il existe encore des exceptions, l'évolution décrite dans ce chapitre a été la règle dans la grande majorité des structures de soins confessionnelles. Nombre d'entre elles ont connu une privatisation croissante dans leur pratique. Celle-ci impose un modèle économique qui va à l'encontre du principe de charité, dont la conséquence la plus frappante est la désertion de ces structures par la grande majorité des pauvres. Ce processus peut encore être inversé, au prix d'une réflexion de fond sur les ambitions et le financement de ce type de structures.

\section{Pour aller plus loin}

Boulenger, D. et Criel, B. (2012). The difficult relationship between faith-based care organisations and the public sector in sub-saharan Africa: The case of contracting experiences in Cameroon, Tanzania, Chad and Uganda. Studies in ITGPress : 236. 
128 - DES IDÉES REÇUES EN SANTÉ MONDIALE

Olivier, J., Tsimpo, C. et Wodon, Q. (2012). Do faith-inspired health care providers in Africa reach the poor more than other providers? Munich: 19.

Olivier, J. et Wodon, Q. (2012). Satisfaction with faith-inspired healthcare services in Africa: review and evidence from household surveys. Munich Pers RePEc Arch: 24 . 


\title{
La micro-assurance santé offre une protection sociale efficace pour les plus démunis
}

\author{
Manuela De Allegri
}

Depuis quelques années, la micro-assurance santé (MAS) reçoit une attention croissante en tant que mécanisme de financement potentiellement utile pour faire progresser la couverture universelle de santé dans les pays à faible et à moyen revenu. Dans les milieux où la capacité du gouvernement à établir des régimes d'assurance sociale ou des régimes fondés sur l'imposition est contrainte par le contexte sociopolitique et économique, la MAS comble une lacune dans la protection sociale de la santé. Elle offre une couverture aux personnes qui ne seraient pas couvertes par une autre forme d'assurance. La MAS applique le concept d'assurance au niveau micro. Elle favorise le partage du risque et la mise en commun des ressources au sein d'une communauté pour faciliter l'accès aux soins et offrir une protection financière par rapport au coût de la maladie. La MAS favorise une solidarité des personnes en santé envers les malades et dissocie le paiement de la date des soins donnés. En pratique, cela signifie que les personnes paient des contributions régulières en espèces (appelées des primes) dans un panier commun. L’argent accumulé est alors utilisé pour couvrir les coûts de soins médicaux lorsqu'une des personnes ayant contribué tombe malade. Selon les contextes, la MAS est aussi décrite comme une assurance-maladie collective, une assurance santé communautaire ou une mutuelle de santé. 
La participation dans ces programmes est habituellement volontaire (sauf dans quelques rares exceptions à travers le monde), ce qui signifie que les membres d'une communauté sont libres de décider de s'affilier ou non à la MAS.

Les écrits scientifiques ont surveillé de près l'expérience de nombreux programmes implantés à travers l'Afrique et l'Asie afin de mesurer les impacts engendrés par la mise en place de MAS. Ils ont conclu que la participation dans les MAS peut mener à un meilleur accès aux soins et fournir au moins une protection partielle par rapport au coût de la maladie.

Il existe cependant un mythe qui persiste dans le dialogue politique concernant la MAS. Ce mythe circule particulièrement parmi les ONG et les donateurs intéressés à promouvoir le développement de ces programmes. Il s'agit de l'idée qui établit que la MAS représente une assurance en faveur des personnes pauvres et suggère que les programmes de MAS fournissent une protection sociale en santé efficace pour les plus démunis. Ce mythe prévaut malgré l'ensemble de preuves à son encontre.

En général, hormis les pays où la participation est soutenue par le gouvernement (comme au Ghana ou au Rwanda), les programmes de MAS ont des faibles taux d'affiliation, au maximum $20 \%$. De plus, la MAS ne réussit pas toujours à atteindre les plus démunis dans une communauté. Les expériences dans des pays aussi divers que le Burkina Faso, la Tanzanie, le Sénégal, l'Inde et le Cambodge indiquent que les plus démunis sont les derniers à s'affilier. Le Ghana et le Rwanda constituent des exemples intéressants sur la manière dont les iniquités dans l'affiliation prévalent, même lorsque la participation est soutenue par le gouvernement. La MAS est relativement efficace pour atteindre les travailleurs du secteur informel, mais seulement s'ils vivent au-dessus du seuil de pauvreté et ont accès à une source de revenu stable. Cela signifie que les programmes sont conçus dans l'intention d'atteindre des grands fragments de populations rurales défavorisées, mais qu'ils finissent par atteindre uniquement une proportion limitée de personnes des communautés cibles, et le plus souvent les moins pauvres.

En outre, l'expérience de beaucoup (mais pas tous) suggère qu'une fois affiliés, les plus démunis sont les derniers à bénéficier de la couverture d'assurance. Des femmes pauvres, affiliées au programme SEWA en Inde, ont continué à avoir un moins bon accès aux soins comparativement aux 
personnes affiliées moins pauvres, même après la mise en place de mesures pour simplifier l'accès aux soins et les procédures de remboursement. Des résultats similaires ont été observés au sein du programme Gonoshasthaya Kendra au Bangladesh, où la participation à la MAS n’a pas éliminé les disparités entre les femmes les plus pauvres et les moins pauvres concernant l'utilisation de services de santé maternels. Les personnes pauvres semblent souffrir de nombreuses privations, telles qu'un accès limité à l'information sur leurs droits et un accès très restreint aux ressources financières pour couvrir la portion des coûts des soins non couverts par le programme, que la MAS toute seule n'est pas capable d'offrir.

Ayant reconnu l'importance de la pauvreté en tant que barrière pour la participation à la MAS, certains pays ont instauré des politiques pour assurer l'inclusion des plus démunis. Par exemple, le Cambodge a instauré des fonds d'équité en santé. Ces fonds ne sont pas encore appliqués de façon efficace dans tous les districts du pays, ce qui signifie qu'une grande proportion des plus démunis n'a toujours pas accès à une protection sociale adéquate en santé. Il y a actuellement des expériences en cours pour améliorer l'intégration entre ces fonds et les programmes existants de MAS. De la même façon, le ministère de la Santé en Tanzanie a pris des dispositions et a alloué des budgets réservés aux districts de santé pour soutenir la participation des ménages à faible revenu dans les fonds de santé communautaire. La mise en œuvre manque cependant d'orientations politiques, probablement à cause d'une difficulté globale de cibler efficacement les plus démunis. Des nombreux organismes travaillent actuellement pour renforcer le ciblage des personnes pauvres afin d'assurer une participation plus équitable dans les fonds de santé communautaire.

En regardant les données probantes, on peut presque perdre espoir et abandonner l'idée d'investir dans le développement des MAS pour aller vers une protection sociale de santé plus grande pour les communautés défavorisées en Asie et en Afrique. Toutefois, heureusement, il existe quelques histoires positives à partager sur la manière dont les obstacles à la participation peuvent être surmontés ainsi que la manière dont les personnes pauvres peuvent être accompagnées pour bénéficier de la couverture d'assurance. Depuis 2007, l'introduction d'une subvention en faveur des personnes pauvres couvrant la moitié des coûts de la prime dans un programme d'assurance-maladie communautaire en milieu rural au Burkina Faso a considérablement diminué les iniquités dans l'affiliation. 
Il est intéressant de noter que la subvention a produit un effet sur l'utilisation de services, faisant en sorte que les personnes pauvres affiliées les utilisent plus que les équivalents non affiliés (bien que de façon moindre que les affiliés moins pauvres). Cela suggère que la campagne de ciblage et l'offre subséquente de subvention de la prime pourraient avoir aidé indirectement à sensibiliser les personnes pauvres concernant leurs droits à l'assurance. De façon similaire, des expériences dans les milieux ruraux de Bihar et d'Uttar Pradesh en Inde démontrent que lorsque les programmes sont conçus depuis le début en accordant une attention particulière et explicite aux personnes pauvres, au statut socioéconomique et à d'autres mesures socioculturelles de la pauvreté et de l'exclusion sociale (par exemple, les castes), ils constituent moins un obstacle pour l'affiliation.

Ainsi, la MAS n'est pas faite en soi pour servir les personnes pauvres, sauf si des mesures de ciblage sont prévues dans sa mise en œuvre. Il faut aborder le développement d'un programme de MAS avec prudence. Les organismes intéressés dans son implantation doivent être préparés à faire face aux faibles taux d'affiliation, au moins pendant les premières années, et être conscients que des stratégies explicites doivent être mises en place depuis le début d'un programme afin d'assurer son efficacité pour les plus démunis. Ces stratégies incluent, entre autres, un ciblage en faveur des personnes pauvres, une subvention de la prime ou des primes ajustées selon le revenu, des informations additionnelles en faveur des personnes pauvres et des campagnes de sensibilisation sur les droits à l'assurance santé.

\section{Pour aller plus loin}

De Allegri, M. (2006). To enrol or not to enrol in Community Health Insurance - Case Study from Burkina Faso. Frankfurt, Peter Lang.

Rösner, H.J., Leppert, G., Degens, P. et Ouedraogo, L. M. (2012). Handbook of Micro Health Insurance in Africa. Berlin, Lit Verlag.

Soors, W., Devadasan, N., Durairaj, V. et Criel, B. (2010). Community Health Insurance and Universal Coverage: Multiple paths, many rivers to cross. Background Paper, 48 to the World Health Report 2010. Geneva, The World Health Organization. 


\title{
Les personnes vivant dans les villes sont privilégiées du point de vue de la santé
}

\author{
Florence Fournet
}

L'urbanisation est sans nul doute le phénomène planétaire le plus marquant de ces dernières décennies. Depuis 2007, la moitié de la population vit dans des villes et en 2030, ce sera les deux tiers. Cette explosion urbaine concernera principalement les pays du Sud. C'est en Afrique que la croissance urbaine sera la plus forte (4,8\% par an), de telle sorte que plus d'un Africain sur deux vivra en ville en 2015, et c'est dans les villes secondaires, moyennes voire petites, que la croissance démographique à venir sera la plus forte. L'urbanisation se réalise aujourd'hui dans un contexte économique difficile et à une vitesse beaucoup plus rapide que ce que l'Europe a pu connaître au XIX ${ }^{e}$ siècle.

Deux visions de la ville se sont toujours opposées, celle d'un Eldorado source de modernité et donc de développement et celle d'un lieu de perdition, délétère et pestilentiel, qui peut nuire à sa population. Par la densité démographique et l'ouverture sur l'extérieur caractérisant les villes, leur hétérogénéité est une évidence qui fait dire à certains qu'il est «des villes dans les villes».

Pourtant, en concentrant une grande part des ressources nationales en matière d'éducation, de santé et plus généralement de services, les villes, en particulier les capitales, ont fait naître l'idée que leurs habitants bénéficieraient de meilleures conditions de vie, et jouiraient dès lors d'une 
meilleure santé. On parle ainsi de privilège urbain. Les citadins seraient de ce fait en meilleure santé que leurs voisins des campagnes, éloignés des structures de soins, mal informés, comme laissés-pour-compte.

\section{Des indicateurs à utiliser avec prudence}

De nombreux indicateurs semblent pencher en faveur de ce privilège urbain. Ainsi, l'espérance de vie serait plus longue en ville, la couverture vaccinale meilleure, l'accès à l'eau potable et à l'assainissement plus généralisé. Mais sur quelles données ces chiffres s’appuient-ils? Quels dénominateurs de population mobilisent-ils dans des villes tentaculaires en perpétuelle croissance au Sud, si mal organisées et où les recensements sont quasi obsolètes avant même d'être publiés? La réalité est-elle donc aussi évidente?

Les statistiques internationales le montrent bien souvent, les urbains ont une espérance de vie plus grande que les ruraux. En France, l'espérance de vie a commencé à s'élever dans les grandes villes (Lyon, Paris, puis Marseille) à partir du milieu du XIX ${ }^{e}$ siècle au fur et à mesure de la construction des égouts et d'une adduction d'eau potable. Si cette relation est en faveur des bienfaits de l'urbanisation, elle n'a malheureusement pas été confirmée par la suite, en Angleterre notamment. Et voilà qu'aujourd'hui, des études menées en Europe suggèrent que la pollution atmosphérique liée aux transports et à l'industrie pourrait réduire l'espérance de vie des citadins.

Au chapitre de la prévention, si la couverture vaccinale est toujours meilleure en milieu urbain, l'Organisation mondiale de la santé notait que dans certaines villes, les enfants pouvaient être moins bien vaccinés que ceux des campagnes, eu égard notamment à la méconnaissance du système de soins des néo-citadins arrivant des campagnes ou de l'étranger.

Dans les villes du Sud, et plus particulièrement dans les quartiers défavorisés, les besoins en eau potable et en assainissement ne sont pas couverts, ou seulement partiellement. Cela entraîne la détérioration de la qualité de la vie et la multiplication des maladies liées à l'eau (maladies diarrhéiques et choléra notamment). À la suite de la privatisation de nombreux réseaux de distribution, le prix élevé de l'eau est devenu un obstacle presque insurmontable pour de nombreux citadins pauvres. Au problème de l'approvisionnement en eau potable s'ajoute celui de l'assainissement. 
La pratique de la défécation en plein air, le rejet incontrôlé des eaux usées et l'inefficacité du drainage des eaux de surface, qui semblent sans conséquence dans les zones rurales peu habitées, se traduisent en ville par la pollution des ressources en eau douce et, ainsi, la recrudescence des maladies hydriques. À Delhi, en Inde, par exemple, la quantité de coliformes fécaux dans le Gange, qui traverse la ville, est multipliée par 3000 entre son entrée dans la ville et sa sortie. Et si des villes comme Ouagadougou au Burkina Faso se sont mises à ramasser, trier, recycler et enfouir leurs déchets, combien d'autres se sont laissé déborder?

\section{Un tableau épidémiologique en mutation}

On sait la ville propice à de nombreux changements de comportements: modification des régimes alimentaires avec l'augmentation de la consommation de protéines et de sucres, accès à une alimentation riche en graisses et en sel, réduction de l'activité physique, accès plus aisé à des produits toxiques (tabac, alcool, drogues), etc. Ces mutations ont des incidences sur le plan sanitaire, notamment l'émergence des maladies non transmissibles ou dites de civilisation comme le diabète, les maladies cardiovasculaires, les cancers, les pathologies mentales. Ces maladies sont la première cause de mortalité dans le monde et elles sont à l'origine de $63 \%$ des décès dans les pays à faible revenu.

L'Organisation mondiale de la santé estime que 366 millions de personnes souffrent de diabète dans le monde et que d'ici 2030, elles seront 552 millions. Cette épidémie est liée à l'occidentalisation des modes de vie, en lien notamment avec le milieu urbain. Dans les pays riches, si les populations les plus pauvres sont les plus affectées, dans les pays à faible revenu, le problème concerne encore la part la plus aisée de la population, même s'il s'étend. De fait, au sein des pays à revenu intermédiaire, les différentes catégories de population sont toutes concernées. Ces pathologies soulèvent de nombreux problèmes quant à leur dépistage, puis leur prise en charge, dans des pays où les traitements ne sont pas toujours disponibles et représentent un investissement souvent très (trop) lourd pour un ménage qui n’a déjà pas forcément les moyens de bien se nourrir.

Dans les pays du Sud, ces maladies dites de civilisation viennent s'ajouter aux maladies infectieuses et parasitaires. En effet, si certains scientifiques ont pu penser que la pathologie urbaine des pays du Sud 
évoluerait à l'image de celle des pays du Nord, cette hypothèse est désormais rejetée: les maladies infectieuses et parasitaires ne disparaissent pas au profit des maladies chroniques et dégénératives. Ainsi, les populations affrontent un double fardeau de morbidité.

En ce qui concerne les maladies infectieuses, les maladies diarrhéiques persistent en lien avec la mauvaise hygiène du milieu, de même que les affections respiratoires, qui affectent tout particulièrement, comme les précédentes, les jeunes enfants. S'agissant de la tuberculose, on observe que son incidence est beaucoup plus élevée dans les grandes villes. En République démocratique du Congo, $83 \%$ des personnes atteintes vivraient dans les villes. À leurs côtés, la peste, la dengue, le paludisme, la maladie du sommeil ou encore la leishmaniose sont entrés dans les villes ou sont à leurs portes, alors qu'on les croyait réservés au monde rural. Sont en cause l'adaptation des vecteurs des campagnes à la ville, le développement des cultures maraîchères qui favorisent le maintien de ces mêmes vecteurs, l'installation de populations qui viennent de la campagne avec leurs parasites, ou encore les conditions de vie dans certains quartiers qui confinent le bétail avec les hommes. Face à ce double fardeau de morbidité, l'offre de soins des villes peut-elle satisfaire les besoins des populations?

\section{Adéquation entre besoins de santé et offre de soins}

Les villes, qu'elles soient du Nord ou du Sud, concentrent une grande part des ressources humaines et matérielles en matière de services. En 2002, en Chine, grâce à l'abondance de l'offre scolaire, pour quatre enfants scolarisés dans le primaire en zone urbaine, trois seulement l'étaient dans les campagnes. Et si la différence semble faible, elle implique néanmoins que plus d'un million d'enfants ruraux n'ont pu accéder à l'école primaire. Abidjan concentrerait $60 \%$ du personnel de santé de la Côte d'Ivoire, tandis qu'Alger disposerait de plus de la moitié des structures de soins du pays.

Ces chiffres masquent cependant des disparités importantes. Ainsi, au Burkina Faso, en 2003, le milieu urbain regroupait en moyenne un tiers du personnel de santé. Or, si ce chiffre s'applique aux infirmiers, cela n'est plus vrai des médecins, qui sont près de $70 \%$ à exercer en ville, essentiellement dans la capitale. Quant aux structures de soins, si $9 \%$ de celles du premier niveau sont retrouvées dans les villes, cela ne vaut pas pour 
les structures confessionnelles et privées, dont la part s'élève respectivement à $77 \%$ et $85 \%$ en ville.

Par ailleurs, la présence d'une offre de soins abondante ne signifie nullement que la population y recourt. Au-delà de son accessibilité physique qui apparaît plus aisée en ville, il ne faut pas minimiser l'importance des obstacles économiques et socioculturels.

La distance aura de l'importance dans les quartiers périphériques, sous-équipés, peu motorisés et mal desservis par les transports en commun, qui seront ainsi touchés par des inégalités territoriales d'accès aux soins. Par ailleurs, si l'offre de soins s'est multipliée dans les villes, le coût des soins dans une structure privée est généralement plus élevé et constitue un réel obstacle aux recours. Enfin, les inégalités culturelles ont aussi leur importance, particulièrement pour les néo-citadins qui peinent à s'approprier la ville. À Marrakech, par exemple, une enquête a montré que les femmes issues de l'exode rural recouraient moins aux soins de santé maternelle que celles nées en ville, essentiellement du fait de situations économiques, mais aussi culturelles défavorables.

\section{Les enjeux de l'urbanisation en santé}

On l'aura compris, la santé des citadins n'est pas forcément meilleure que celle de leurs voisins ruraux. Il n'est pas de privilège urbain en santé qui soit partagé par tous.

L'hétérogénéité des villes est productrice d'inégalités sociales, économiques et sanitaires, et certaines populations urbaines peuvent être en moins bonne santé que les populations rurales. La ville n'a pas aboli l'ensemble des obstacles qui freinent l'accès aux soins au plus grand nombre, malgré la multiplicité de l'offre de soins et sa diversité.

Dans la mesure où le monde de demain sera urbain, il devient urgent de faire des villes une priorité sanitaire. N'oublions pas qu'aucune référence à la santé urbaine n’a été faite en 1978 lors de la Conférence mondiale d'Alma Ata consacrée aux soins de santé primaires. Il aura fallu attendre l'année 2010 pour inaugurer la première année de la santé urbaine par l'OMS. 
138 - DES IDÉES REÇUES EN SANTÉ MONDIALE

\section{Pour aller plus loin}

ICSU (2011). Report of the ICSU planning group on health and wellbeing in the changing urban environment: A systems analysis approach. International Council for Science, Paris.

OMS et ONU-HaвiтAт (2010). La face cachée des villes: mettre au jour et vaincre les inégalités en santé en milieu urbain. Rapport mondial, Genève.

Salem, G. (1998). La santé dans la ville. Géographie d'un petit espace dense: Pikine (Sénégal). Karthala, Paris. 


\title{
C'est sur les routes des pays les plus pauvres qu'on meurt le plus
}

\author{
Emmanuel Bonnet
}

Si la prise de conscience du fardeau de santé publique représenté par l'insécurité routière est récente, l'idée reçue établissant que ce sont les pays les plus pauvres, notamment en Afrique, qui connaissent une mortalité routière importante est plus ancienne. Cette mortalité serait notamment associée à une mauvaise formation à la conduite, une corruption forte pour l'acquisition des permis de conduire et une vétusté des véhicules.

Tout d'abord, de quoi parle-t-on lorsque la mortalité et la morbidité sur la route sont évoquées? Les experts en transport parlent d'accidents de la circulation pendant que les experts en sécurité routière et en santé évoquent les traumatismes issus de la circulation routière. Ces derniers font en effet l'hypothèse que tous les traumatismes liés à la sécurité routière sont évitables par la mise en œuvre de mesures de prévention face aux cinq principaux facteurs de risque que sont la conduite en état d'ivresse, le non-usage de la ceinture de sécurité ou du casque, les excès de vitesse et l'absence de protection des enfants.

L’idée reçue repose également sur des perceptions individuelles et collectives influencées par des comportements sur les routes. En Afrique de l'Ouest, la distinction entre les circulations intra et interurbaines est très importante. Les véhicules qui y circulent sont différents ainsi que les vitesses pratiquées. En ville, la mixité est forte, elle est composée de deux- 
roues motorisées, de bicyclettes, parfois d'animaux, de voitures, de camions, et les vitesses sont relativement lentes. En interurbain, ce sont les voitures, les camions et les bus qui dominent. Ces véhicules pratiquent des vitesses importantes en raison d'un trafic peu dense. En général, dans les villes ouest-africaines, les deux-roues et les voitures cohabitent dans une forte densité. Le respect des feux tricolores ou des arrêts est souvent aléatoire. Les perceptions collectives et individuelles sont influencées par l'encombrement, la circulation désordonnée et l'incivilité. Pour les liaisons interurbaines, c'est la vitesse et le comportement des grands véhicules comme les bus ou les camions qui génèrent des perceptions négatives. Des accidents graves avec un nombre de décès importants surviennent fréquemment et produisent des perceptions collectives sur la dangerosité des routes africaines.

\section{Des données difficilement comparables}

Des rapports récents estiment à 1,2 million les morts et 50 millions les blessés sur les routes dans le monde. La majorité de ces décès (92\%) surviendrait dans les pays à revenus faibles et intermédiaires, alors qu'ils ne représentent que $52 \%$ du parc automobile. L'Afrique est le continent qui apparaît le plus touché. Les décès issus de la circulation routière représenteraient la cinquième cause de mortalité, alors qu'elle n'est qu'au dixième rang au niveau mondial. Dans tous les cas, la mortalité et la morbidité liées aux traumatismes de la circulation routière augmentent considérablement dans les pays à revenus faibles et intermédiaires, alors qu'elles tendent à régresser dans les pays à revenus élevés. Or, la diminution du nombre de morts en France, par exemple, se réalise lentement par de nombreuses législations, des observatoires, une amélioration des véhicules et des infrastructures. La situation dans les pays à revenus intermédiaires et faibles est tout autre; la simple mesure du nombre d'accidents est déjà difficile.

Si les perceptions collectives, la connaissance empirique et les rapports mondiaux convergent pour annoncer que la plus forte mortalité due à la circulation routière est dans les pays les plus pauvres, il est nécessaire de comprendre comment ces conclusions sont produites, sur quelles preuves elles reposent.

Depuis 2004, l'OMS et la Banque mondiale ont lancé des mesures d'évaluation pour connaître et suivre les évolutions en matière de sécurité 
routière dans près de 195 pays. Entre 2009 et 2013, on note que les taux de mortalité les plus hauts surviennent dans les pays à revenus faibles et intermédiaires, en particulier en Afrique. La situation entre 2009 et 2013 se serait améliorée dans les pays à hauts revenus, alors qu'elle se serait détériorée dans les pays à revenus faibles et intermédiaires. Ces conclusions sont issues de données collectées par l'intermédiaire de relais locaux dans les directions des services de sécurité routière nationale. Les données seraient ensuite vérifiées et validées par les agents de l'OMS. Dans le même temps, l'OMS mentionne que la standardisation des données sur les traumatismes et les modes d'acquisition des données doivent être améliorés.

La collecte des données et la production de résultats sont des éléments clés pour comprendre la situation dans un pays, et plus globalement, sa position dans un ordre mondial de la sécurité routière. Les comparaisons à ces échelles peuvent en effet être difficiles dans la mesure où il n'existe pas de mode de collecte standardisé, ni de contrôle de la qualité des données. À titre d'illustration, je présenterai l'organisation et la production des données au Burkina Faso, un pays à revenus faibles pour lequel des statistiques sont produites dans les rapports mondiaux.

\section{Le Burkina Faso, un pays à faible revenus}

Au Burkina Faso, la collecte des données relatives aux traumatismes issus de la circulation routière n'est pas systématique. La procédure classique, dans la ville de Ouagadougou, repose sur la prise en charge des victimes graves par la Brigade des Sapeurs Pompiers. Ceux-ci acheminent les victimes aux urgences médicales dans le principal Centre hospitalier universitaire (CHU). Les procès-verbaux d'intervention sont saisis inégalement par la Brigade. Le CHU renseigne l'entrée de la victime comme un accidenté de la route dans le registre. Les registres sont ensuite compilés au ministère de la Santé pour produire des statistiques nationales. Toutefois, d'autres acteurs peuvent intervenir et produire des données relatives aux traumatismes et décès issus de la circulation, comme la police nationale ou la gendarmerie.

En 2012, l'observatoire de la Sécurité de Ouagadougou recensait près de 68 décès et 11023 blessés, alors que le CHU enregistrait 5122 victimes évacuées, dont 92 décès dans la seule ville de Ouagadougou, et le ministère de la Santé en comptabilisait 104 (dans les services d'hospitalisation) au 
niveau national! D’autres sources convergeraient pour annoncer 500 morts et 6000 blessés pour l'ensemble du Burkina Faso. Le rapport de l'OMS dénombrait 966 morts et proposait une estimation à 4566 morts dans le pays. Si les méthodes employées pour le rapport mondial sont parfaitement décrites, si les sources sont mentionnées (ministère des Transports), elles ne pourraient en aucun cas être mises en œuvre sur le territoire burkinabè.

À ce jour, au Burkina Faso, il n'existe pas d'organe administratif qui synthétise les données relatives aux accidents de la route et aux traumatismes. Plusieurs services collectent irrégulièrement des informations sans les partager et les croiser avec les services de l'État. De même, le ministère de la Santé n'est pas en mesure de décomposer la mortalité issue de la circulation routière.

La publication de tels chiffres est donc surprenante et contribue à alimenter des idées reçues qui reposent sur des perceptions issues du quotidien, de stéréotypes et confortées par des publications faisant la preuve d'une situation nationale qui s'avère pourtant impossible.

Dans ces mêmes rapports, il est fait mention des politiques publiques et des législations en vigueur afin de mesurer l'engagement des pays à suivre les recommandations des experts pour réduire les cinq risques majeurs. Ainsi, toujours au Burkina Faso, s'il existe une limitation de vitesse dans les villes et les zones rurales, il y a peu de moyens de contrôle. On dénombrait en 2011 seulement trois radars à visée directe, essentiellement utilisés dans la capitale et son environnement proche. Le rapport mentionne une loi sur l'alcool au volant, sans détail particulier. Elle est inexistante dans le pays. Concernant la ceinture de sécurité, la loi sera mise en œuvre en 2014 et ne semble pas encore faire l'objet de contrôles et de prévention. Enfin, si la loi sur le port du casque existe, elle est à ce jour suspendue en raison d'une farouche opposition de la population qui s'est mobilisée contre sa mise en place en avril 2005. La connaissance des réglementations en vigueur publiées dans ce rapport est donc juste, mais elle manque de vérification concernant leur application.

La comparaison entre tous les pays du monde est difficile, car la production de données est inégale et souffre d'un manque d'intérêt pour certaines nations. Dans un pays pauvre, la collecte, la maintenance et le traitement des données représentent aussi un coût non négligeable et non prioritaire. Ainsi, dire que ce sont les pays à revenus faibles et intermé- 
diaires, en particulier ceux d'Afrique, qui détiennent les plus forts taux de mortalité sur la route est abusif. S'il paraît possible que cette mortalité soit forte et qu'elle soit l'une des principales chez les jeunes, il est impossible de la chiffrer précisément, au risque d'exagérer et d’alimenter des idées reçues.

Les traumatismes et la mortalité issus de la circulation routière deviennent des fardeaux pour les pays à revenus faibles et intermédiaires, en particulier en Afrique. Au-delà du nombre de morts ou du taux de morbidité, ce fardeau de santé publique représente un coût économique et social majeur. La nécessité de construire des observatoires, couplés à des accompagnements réglementaires et surtout des mesures de prévention, est indispensable. C’est avant tout par la volonté des États, la sensibilisation et la formation des populations que la prise de conscience collective pour la sécurité routière sera efficace et contribuera à diminuer la perte de vies humaines et les handicaps dus aux problèmes de circulation routière.

\section{Pour aller plus loin}

Missimikim, M. (2012). Road safety in the WHO african region. WHO.

ORgANISATION MONDIALE DE LA SANTÉ (2004). Rapport mondial sur la prévention des traumatismes dus aux accidents de la circulation. Genève, Organisation mondiale de la santé.

World Health Organization (2013). Global status report on road safety 2013: Supporting a decade of action. Geneva, World Health Organization (WHO). 


\section{Les femmes meurent plus tard que les hommes et sont plus malades}

Pierre Ä̈ach

Les femmes forment un groupe social dominé, quel que soit le pays envisagé. Et pourtant, contrairement à ce qui est observé pour les autres groupes sociaux (classes, catégories sociales), elles vivent plus longtemps que les hommes tout en étant plus malades qu'eux. La mortalité supérieure des hommes est généralement connue du grand public. De plus, les recherches montrent que les femmes sont plus malades et de ce fait, comparativement aux hommes, elles utilisent plus le système de santé. On est en fait face à deux réalités qui sont admises sans que leur caractère contradictoire ne soit souligné ou même perçu.

Ces deux réalités, mortalité des hommes plus forte que celle des femmes et morbidité (nombre et nature des maladies) plus élevée chez les femmes, sont en effet en contradiction dans la mesure où si on meurt dans un groupe (ici les hommes) en moyenne plus tôt, c'est qu'on est plus malade et plus gravement que dans un autre groupe (les femmes).

Or, c'est précisément ce qui n’apparaît pas dans les données internationales. Les femmes ont une espérance de vie à la naissance (nombre moyen d'années de vie) supérieure à celle des hommes. Selon l'Organisation des Nations Unies, cet écart serait par exemple de 4,1 ans au Royaume-Uni, de 11,7 ans en Russie, de 3,5 ans en Inde et en Indonésie, de 1 an en Afrique du Sud, de 3,6 ans au Soudan, de 4 ans en Égypte, etc. 
De plus, les femmes déclarent davantage de maladies et consultent plus souvent. En France, par exemple, en 2009, 39 \% des femmes contre $35 \%$ des hommes ont déclaré au moins une maladie chronique. Par exemple, en France en 2009, pour les personnes âgées de 45 à 54 ans, 33\% des femmes contre $28 \%$ des hommes consultent les services de santé.

Nous sommes donc placés devant un double paradoxe: les femmes, groupe social généralement dominé, connaissent une mortalité inférieure à celle des hommes et déclarent cependant davantage de maladies. En outre, elles se disent en plus mauvais état de santé et fréquentent plus les services de santé que les hommes.

Pour le premier paradoxe, cette mortalité plus faible, en particulier dans les pays économiquement riches, paraît en contradiction avec le statut des femmes en tant que groupe social dominé, tant économiquement que symboliquement. En effet, la réalité sociale d'hier et d'aujourd'hui montre un rapport logique entre la position dans l'échelle sociale et l'espérance de vie. Plus vous êtes en haut de l'échelle sociale et plus longue sera votre espérance de vie, même si cela n'a pas toujours été le cas. Jusqu'à une date encore récente, les femmes ne mouraient pas plus tardivement que les hommes, en tout cas pour un certain nombre de groupes d'âge. Les raisons évoquées concernent les causes de mortalité principales qui décimaient hommes et femmes, riches et pauvres, de façon égale (maladies infectieuses et épidémies). À l'époque, nous faisions en effet face à l'impuissance des moyens thérapeutiques et de la science médicale, ce qui venait s'ajouter aux risques de mortalité maternelle très importants. Dans une certaine mesure, ce schéma épidémiologique reste encore à l'œuvre dans certaines régions du monde.

Mais avec l'amélioration des conditions de vie, de l'hygiène publique, avec l'apparition de moyens thérapeutiques et de prévention efficaces, un nouveau modèle épidémiologique s'est mis en place. Ainsi, nous avons vu apparaître de nouvelles causes, bien plus en rapport avec le comportement individuel, le niveau de vie et le mode de vie: maladies dégénératives chroniques comme les maladies cardiovasculaires et le cancer, qui représentent aujourd'hui les principales causes de mortalité, en particulier le cancer, qui est devenu la première cause de mortalité chez les hommes en France. Pour comprendre le premier paradoxe, il faut donc non seulement considérer le nouveau tableau épidémiologique des causes de décès, mais également les facteurs qui jouent pour chacune d'entre elles. 
Le deuxième paradoxe se présente ainsi: la morbidité des individus est étroitement liée à sa mortalité, car on meurt surtout de maladies, mais dans le cas des femmes, c'est l'inverse que l'on observe. La logique et l'opinion courante voudraient pourtant que les individus les plus malades soient ceux qui meurent tôt. Pourtant, les femmes ont une morbidité déclarée et diagnostiquée plus forte que celle des hommes. Ce paradoxe pourrait s'expliquer par le fait que la morbidité dont il est question est celle recueillie auprès de personnes interrogées ou examinées, sans qu'une analyse biologique soit faite pour évaluer le risque de mortalité que comporte chaque maladie.

Dans les deux faces de ce double paradoxe, on a affaire à une réalité qui est dans une certaine mesure contraire au sens commun et, surtout, contraire à un raisonnement logique, dans lequel la santé ne se présente pas comme l'expression de l'accessibilité aux services de soins ou comme celle d'un patrimoine génétique programmé. Les maladies, troubles et symptômes morbides n'expriment pas tous le même risque vital et certains même ne présentent aucun caractère de gravité. Cette distinction est capitale, car elle est au cœur du problème posé: mortalité faible et morbidité élevée, mortalité forte et morbidité faible. Finalement, tout se passe comme si les femmes avaient l'astuce d'avoir des maladies non fatales. En revanche, les hommes auraient des maladies plus graves.

Donc, leur sur-morbidité déclarée ou diagnostiquée ne signifie pas que les femmes sont plus malades que les hommes. S'agissant de causes dont on meurt, les rapports entre les femmes et les hommes sont très proches de ceux que l'on trouve dans la mortalité. Plus on est dans le domaine psychologique, fonctionnel, voire psychosomatique, et plus l'écart entre les femmes et les hommes est élevé. Plus on s'éloigne des troubles présentant un risque de mortalité, plus le rapport entre les femmes et les hommes augmente. Il faut trouver ailleurs que dans l'état de santé réel des hommes et des femmes l'explication de ce phénomène. Elle réside dans la construction du rapport au corps différenciée des hommes et des femmes, ainsi que dans le partage des rôles sociaux. Ce qui différencie les hommes des femmes concerne essentiellement la violence symbolique qui s'exerce dans le rapport de domination des hommes sur les femmes. Ce n'est pas parce qu'elles subissent davantage de violences physiques et qu'elles sont soumises à des pressions psychologiques, difficiles et souvent douloureuses, que les femmes sont plus malades. Ce 
phénomène, que l'on retrouve dans la plupart des pays où de telles données sont recueillies, n'est ni négatif ni positif, ni stigmatisant ni valorisant.

\section{Pour aller plus loin}

AïACH, P. (2001). Femmes et hommes face à la mort et à la maladie, des différences paradoxales. In Femmes et hommes dans le champ de la santé, approches sociologiques, Aї̈ACH, P., CÈBe, D., Cresson, G., Philippe, C. (dir.), ENSP.

Aïach, P., Philippe, A., Leclerc, A., Vennin, M. et Cèbe, D. (1979). Les femmes sont-elles plus malades que les hommes? Revue française de la Santé Publique, 4.

Hunt, K. et Sweeting, H. (1996). Gender differences in health: are things really as simple as they seem? Social Sciences and Medicine, vol. $42, \mathrm{n}^{\circ} 4$. 


\title{
La réduction des inégalités sociales de santé passe principalement par des actions en faveur des plus vulnérables
}

\author{
Eric Breton
}

Les inégalités sociales de santé sont celles qu’on ne peut attribuer aux caractéristiques biologiques, physiologiques et génétiques des différents groupes qui composent une population. L'idée que leur réduction passe nécessairement par des interventions en faveur des plus vulnérables est fortement enracinée dans le discours et l'action publique et guide l'essentiel des programmes déployés par les organisations caritatives, et aussi l'approche privilégiée par les gouvernements de tradition démocratique libérale (Australie, Canada, États-Unis, Royaume-Uni) dont les programmes sociaux visent à offrir un filet de sécurité aux individus qui se retrouvent dans des circonstances matérielles et de ressources en deçà de ce qui est jugé acceptable par la société. Je défends pour ma part l'idée que la réduction des inégalités sociales de santé relève davantage d'une approche qui doit concerner toute la population que d'une stratégie ciblant les groupes dits à risque ou vulnérables.

\section{L'épreuve de la réalité empirique}

L'idée même de groupes vulnérables suggère une dichotomie qui, tout comme les lignes qui tracent les frontières sur une carte du monde, n'existe 
pas dans la réalité du paysage sociosanitaire. Agir en faveur des individus dits vulnérables exige tout d'abord de définir ce groupe pour ainsi délimiter les contours de l'action publique. Qui inclut-on et sur la base de quels critères? Parle-t-on d'une vulnérabilité liée à une carence de revenus, de diplômes, de soutien social on encore de services? Pour les identifier, l'État se dote de critères basés sur des calculs rigoureux, mais qui ne peuvent échapper à un certain arbitraire. Par exemple, les programmes de revenu de solidarité active (France) ou de l'aide sociale (Québec) établissent une série de conditions pour se prévaloir de l'aide financière. Mais ultimement, la ligne tracée entre le groupe des bénéficiaires de l'aide et des nonbénéficiaires tient davantage du degré de solidarité/générosité dont fait preuve l'État que de conditions objectives qui permettraient d'établir que l'aide est absolument nécessaire à certains individus/familles, alors qu'elle ne le serait pas pour d'autres, qui sont jugés moins précaires mais qui pourtant présentent un niveau de ressources à peine plus enviable.

Mais cette distinction entre personnes vulnérables et non vulnérables est sérieusement remise en question lorsqu'on considère la distribution des ressources, choix, comportements et indicateurs de mortalité/morbidité au sein de la population en fonction de la catégorie socioprofessionnelle. Ainsi, on observe la même distribution en escalier de l'espérance de vie ou du risque de mortalité et de morbidité. Cet escalier que l'on désigne par l'expression gradient social est aussi observable sur les facteurs de risque (tabagisme, sédentarité, carence en fruits et légumes, addictions) et les déterminants en amont de ces facteurs tels le soutien social, les conditions de logement et d'emploi. Les personnes se situant dans la catégorie des cadres et professions intellectuelles constituent un groupe présentant en moyenne des indicateurs toujours plus favorables que ceux des professions intermédiaires, employés, ouvriers et sans emploi. Notre perspective des inégalités ne peut donc faire autrement qu'évoluer devant le constat de l'existence de ce gradient. Car s'il est injuste que les ouvriers vivent en moyenne moins longtemps que les cadres, il est également injuste que les individus regroupés sous la catégorie socioprofessionnelle professions intermédiaires présentent une espérance de vie plus courte que ceux de la classe des cadres.

Ainsi, face à la réalité des données populationnelles examinées en fonction de la hiérarchie sociale, cette vision dichotomique apparaît non seulement inappropriée, mais porteuse d'un préjugé implicite qu'il y a 
chez les personnes incluses dans des groupes dits vulnérables quelque chose qui les distingue du reste du corps social. Cette conception d'un eux-nous devrait donc plutôt laisser place à un nous multiple. Autrement dit, une population dont les conditions quotidiennes d'existence de ses individus, et donc ultimement leur santé et leur bien-être, sont fortement déterminées par leurs positions dans l'organisation sociale.

\section{Les limites des approches centrées sur les groupes vulnérables}

Si l'injustice ne peut uniquement être envisagée comme émergeant de la comparaison entre deux groupes (eux et nous) et qu'il faut plutôt réfléchir en fonction du gradient social, il en découle que ce gradient doit aussi devenir une cible de l'action. Je dis bien «aussi» puisqu'il ne s'agit pas de plaider pour une fin des services, programmes et politiques destinés aux plus vulnérables, ce qui constituerait un déni du devoir d'assistance aux personnes dont la santé et le bien-être sont en danger. Cependant, limiter l'action publique uniquement à ce groupe est critiquable, pour au moins quatre autres raisons:

1. agir uniquement sur les individus dits vulnérables, c'est généralement se limiter à intervenir sur les symptômes plutôt que sur les causes. Par exemple, développer un programme d'aide au logement pour les personnes sans domicile fixe est peu susceptible d'amener des changements aux conditions structurelles qui déterminent l'offre de logement abordable;

2. on écarte de nos actions d'autres groupes moins vulnérables, mais dont la fragilité les expose (ou leurs descendants) au risque de basculer à leur tour du côté des plus vulnérables;

3. les programmes qui visent exclusivement les groupes dits vulnérables sont généralement mal financés et sujets aux aléas des choix politiques. Comme les membres de ces groupes ont peu de voix, pour ne pas dire aucune, dans le processus démocratique, il est aisé de leur retirer le peu qu'ils ont pour réduire par exemple les dépenses publiques;

4. enfin, soulignons que ces programmes contribuent à stigmatiser les individus qu'ils visent, en les affublant justement de l'étiquette vulnérables. Devoir prouver par une déclaration de ressources sa pau- 
vreté pour ainsi accéder aux services, programmes et aides contribue à construire une citoyenneté de seconde classe.

\section{Pour des mesures universelles proportionnées}

Il apparaît donc souhaitable de penser l'action publique pour réduire les inégalités sociales de santé et de bien-être, non plus uniquement comme action sur les groupes vulnérables. Il faut surtout envisager l'action publique sur la population dans son ensemble, ce qui permettrait d'agir sur le gradient social. L'épidémiologiste Geoffrey Rose a démontré que les comportements (le tabagisme, la consommation d'alcool ou de sel, par exemple) ou les indicateurs de santé (la tension artérielle, le cholestérol sanguin, par exemple) d'un groupe à risque sont intimement liés à ceux que l'on retrouve pour l'ensemble de la population. Par exemple, en comparant la consommation moyenne d'alcool par habitant de deux pays, on retrouvera pour le pays avec la consommation moyenne la plus faible un contingent moins important d'individus dépassant un seuil annuel donné de $\mathrm{X}$ litres d'alcool que pour la population avec une consommation moyenne plus élevée. Il apparaît donc plus rentable d'agir sur les facteurs liés à la consommation d'alcool pour toute la population (par exemple par des mesures sur l'accès au produit telles que le prix, le nombre de points de vente, les heures d'ouverture, etc.) que de se limiter à des interventions auprès des consommateurs excessifs.

Cela dit, si en termes coûts-efficacité l'approche prônée par Rose est nettement plus rentable, il n'est pas rare qu'un changement de la moyenne populationnelle s'accompagne d'une augmentation des inégalités. Les écrits scientifiques comptent nombre d'exemples de programmes qui, tout en générant des effets positifs pour les classes moyenne et supérieure, n'en ont eu aucun pour les classes inférieures, ce qui s'explique par un gradient social de la capacité de convertir les apports et opportunités d'un programme en gains de santé ou de bien-être. Notons que cet effet pervers des approches populationnelles est plus susceptible de s'observer si le travail de transformation porté par le programme ne vise que les caractéristiques individuelles (niveau de connaissance, attitudes ou compétences) et non les causes structurelles.

Il serait donc judicieux que les actions s'organisent selon le principe de la mesure universelle proportionnée, autrement dit, par le déploiement 
d'actions dont le bénéfice sera ressenti dans toutes les catégories socioprofessionnelles, mais tout en ayant des effets plus importants en bas de l'échelle sociale.

Les mesures universelles proportionnées demeurent peu étudiées. Cette situation n'est pas attribuable à leur caractère novateur, mais plutôt au fait regrettable qu'on évalue rarement les effets des interventions en santé publique par classe sociale. Quoi qu'il en soit, parmi les mesures universelles aux effets proportionnés avérés, on peut distinguer deux types de stratégie. Le premier type regroupe les interventions dont les propriétés intrinsèques font qu'elles génèrent des ressources dont la traduction en bénéfices est plus forte en descendant l'échelle sociale parce que ces ressources suivent justement un gradient social. Par exemple, les stratégies de prévention de la carie dentaire par l'ajout de fluor à l'eau potable se sont traduites par un gradient social de la carie chez les enfants moins élevé dans une ville comme Newcastle (où il y a apport de fluor) que celui observé à Manchester, qui n'avait pas adopté cette mesure.

L'offre d'un service à toute la population peut aussi générer des effets proportionnés aux besoins des différents groupes. Citons le cas, en Alsace, du programme ICAPS visant à accroître le niveau d'activité physique chez les jeunes collégiens. Comme la ressource transport est inégalement distribuée selon un gradient social, l'offre d'un service de transport après les activités parascolaires a permis aux étudiants de ne pas partir tout de suite après leurs cours et ainsi de participer aux activités. Notons que cette offre de transport n'a eu qu'un faible impact sur le niveau d'activité physique des jeunes dont les familles avaient les ressources pour les conduire à des activités sportives en fin de journée.

Un deuxième type de stratégie par mesure universelle proportionnée consiste à enrichir une mesure universelle par une série d'actions, de ressources (de services, par exemple) reflétant les besoins spécifiques des membres des différentes classes sociales. Ainsi, un programme universel de visites postnatales peut offrir un accompagnement de base à toutes les familles tout en incitant le professionnel qui fait la visite à mettre en place d'autres actions et services pour celles éprouvant des difficultés spécifiques, notamment sur la question de l'emploi, du logement, de la formation ou des problèmes d'accès aux soins. Autre exemple, en Angleterre, les services d'aide au sevrage du tabac constituent une mesure universelle (donc accessible à tous les fumeurs) de la stratégie nationale de lutte contre 
le tabagisme. Les autorités ont toutefois noté que les fumeurs des quartiers défavorisés y avaient moins recours, un constat qui a donné lieu au renforcement des ressources allouées à ces quartiers. L'évaluation de cet effort additionnel a montré que les services rejoignaient une proportion plus grande de fumeurs des quartiers défavorisés que de ceux des autres quartiers, ce qui constitue en quelque sorte un exemple de discrimination positive.

Enfin, si on ne peut nier l'importance de déployer des actions en faveur des populations les plus défavorisées, ce ciblage sur un groupe spécifique est peu susceptible de modifier le gradient social de la santé. C'est pourquoi une stratégie orientée vers des mesures universelles proportionnées apparaît plus prometteuse. Mais son déploiement exige au préalable d'investiguer les causes du gradient pour un choix, comportement ou indicateur sociosanitaire donné. Il faut en effet pouvoir répondre à la question: quel est l'avantage ou le désavantage dont l'importance varie en fonction de la position des individus dans la hiérarchie sociale? Les modèles explicatifs des inégalités sociales de santé suggèrent qu'émergeront des explications concernant l'exposition (aux facteurs de stress, conditions environnementales, etc.) ou les ressources (information, soutien social, revenus).

\section{Pour aller plus loin}

Potvin, L., M. J. Moquet et A. M. Jones (dir.) (2010). Inégalités sociales de santé. Guide d'aide à l'action. Paris, INPES.

Ridde, V. et A. Guichard (2008). Agir pour réduire les inégalités sociales de santé: aporie, épistémologie et défis. In Lutter contre les inégalités sociales de santé, politiques publiques et pratiques professionnelles, C. Niewiadomski et P. AїACH (dir.), Rennes, Edition EHESP : 57-80.

Rose, G.A. (1992). The strategy of preventive medicine. Oxford, New York, Oxford University Press. 


\title{
Les migrants sont par nature vulnérables
}

\author{
Sandrine Musso
}

Parmi les prêts-à-penser au sujet des migrants, leur vulnérabilité singulière en matière de santé est parfois désignée comme constitutive, allant jusqu’à faire de la migration la métaphore d'une maladie. Les nombreuses embûches auxquelles ont à faire face certaines des personnes engagées dans une trajectoire migratoire - du contexte de départ à celui des routes qui mènent à la destination désirée jusqu'à la société dite d'accueil peuvent effectivement affecter leur santé à des échelles diverses. Avec 214 millions de migrants internationaux en 2009 ( $3 \%$ de la population mondiale) et l'apparition de zones de transit inédites, la mondialisation humaine implique un effacement relatif des catégories qui avaient permis par le passé d'analyser les migrations. Les distinctions ne sont plus si nettes entre pays de départ, pays d'accueil et pays de transit, certains d'entre eux étant les deux ou les trois à la fois (Mexique, Turquie, Maroc), ce qui renvoie à leur localisation entre des régions aux caractéristiques politico-économiques marquées par l'inégalité. À l'échelle mondiale, un tiers des migrants se déplace des pays en développement (sud) vers les pays développés (nord); un tiers, du sud vers le sud; et le dernier tiers, du nord vers le nord.

La mondialisation n'a pas, loin s'en faut, aboli les frontières: elle est même la scène de leur renforcement. La surveillance électronique par satellite des forêts tropicales et des aires désertiques se met en place, 
développant ainsi une véritable économie politique de la frontière, et transformant en parcours $d u$ combattant certaines trajectoires migratoires. Pas un jour sans que ceci ne produise des morts: l'imaginaire et le désir d'ailleurs, l'aventure du départ, tout comme les inégalités globales que traduisent les mobilités, ne sauraient être endigués par les forteresses contemporaines. Sur cette toile de fond, qu'en est-il donc des enjeux de santé des migrants et de la manière dont ils sont rituellement formulés?

\section{Des pauvres qui partent dans des pays riches, notamment pour s'y faire soigner}

Cette première idée reçue est en contradiction avec les données sur les migrations internationales. Sur les 214 millions de migrants internationaux, l'écrasante majorité n'est pas issue des catégories les plus pauvres de la société dont ils émigrent. Migrer coûte cher et demande des forces, c'est pourquoi ce sont ceux et celles qui sont en meilleure santé qui partent: c'est le fameux effet favorable aux migrants ("healthy migrant effect») dont nombre d'études des migrations internationales rendent compte. C'est en revanche après plusieurs années d'installation dans la société dite d'accueil que cet état de santé connaît une dégradation: les conditions d'accès au travail, au logement sont par exemple des déterminants de santé en lien avec les discriminations.

La santé est en outre très rarement un motif de migration, alors même que le droit aux soins peut être le dernier «droit d'avoir des droits». Les flux de personnes engagées dans des quêtes de soins peuvent par ailleurs être appréhendés sous la catégorie de tourisme médical. A l'instar des mouvements globaux des migrations internationales, celui-ci recouvre des mobilités disparates (sud-sud, nord-sud, nord-nord, sud-nord) façonnées par de nombreux paramètres: l'accès à la procréation médicalement assistée selon les critères d'éligibilité des postulant-e-s et les mobilités qu'il entraîne est un exemple parmi d'autres.

Par ailleurs, une dimension importante des mobilités contemporaines concerne non pas les personnes engagées dans une quête de soins, mais les personnels de santé des pays des suds, tandis que les analyses de la "global care chain» éclairent la part constituée par les migrants sur le plan du «care» (soins aux personnes âgées et aux enfants) dans le monde contemporain. 


\section{Il y a des obstacles culturels à l'accès à la prévention et aux soins}

Alors que les dimensions culturelles sont spontanément évoquées dans les enjeux qui lient migrations et santé, force est de constater que le trait saillant des phénomènes migratoires aujourd'hui est la place que la loi et le droit occupent. Ceci à la fois dans la formulation des projets de migration, et à l'échelle des conditions sociales d'existence au sein des sociétés dites d'accueil. Les discriminations administratives et juridiques affectent l'accès à la prévention et aux soins de certains migrants, notamment de ceux qui ne disposent pas de statut légal sur le territoire, lesquels constitueraient entre $10 \%$ et $15 \%$ des migrants internationaux. Ces discriminations ont des effets sur les modes de recours comme sur l'expérience des institutions de santé, du fait de la peur de l'expulsion ou du rejet.

La maladie implique aussi une reformulation du projet migratoire, dont elle peut symboliser l'échec. Faire face à l'annonce d'une maladie grave, dont les traitements n'existent pas dans le pays d'origine, amène à reconsidérer sur le long terme un séjour initialement projeté comme temporaire. Affronter les conséquences sociales et économiques d'une affection a des effets, par ailleurs, sur l'assignation à la réussite et la mission de soutien au pays d'origine dont est investi celui qui est parti vis-à-vis de ceux qui sont restés.

Mais là encore, un ensemble d'inégalités et de variations sont remarquables. Les discriminations d'accès au statut légal, notamment pour des raisons de santé, sont enchâssées à un ensemble de représentations. Ces dernières sont façonnées tant par l'Histoire que par des enjeux politiques actuels, elles impliquent un travail de tri opéré entre migrants qui le méritent et ceux qui ne mériteraient pas l'accès à la régularisation de leur situation, soit en raison de la nature de leur pathologie, soit en raison de la société dont ils sont originaires.

La dimension culturelle des faits de maladie et de santé, en dépit de son importance indéniable, n'est pas la seule dimension contextuelle à prendre en compte en termes de santé des migrants. Cette perception de la dimension culturelle peut aussi souffrir de présupposés partiels et partiaux, en ne pensant qu'une partie de celle-ci: l'exemple souvent évoqué des rapports de genre et du refus de l'interaction avec un médecin homme pour les femmes originaires de certaines sociétés est le parangon. C’est une double assignation que peuvent vivre les femmes migrantes dans la 
société de destination, entre les normes en usage dans leur société d'origine et les clichés victimaires à leur endroit dans les sociétés d'accueil. C'est en somme sur le plan relationnel, et non en postulant que «la culture, c'est celle de l'autre», qu'il s'agit d'appréhender la dimension culturelle.

\section{Adapter les services de santé et promouvoir l'accès des migrants aux soins}

Les accès à la prévention et aux soins des migrants peuvent entrer en dissonance avec des politiques ayant pour visée la «régulation des flux migratoires», voire l'ordre public. Or, tel n'est pas le prisme sous lequel la santé des migrants a longtemps été envisagée dans les manuels de santé publique. La classique distinction entre les pathologies d'importation, d'acquisition et d'adaptation a fait l'objet de critiques en raison des deux logiques qui la sous-tendent. D’une part, celle de la discrimination, en instituant une clinique à part, et d'autre part, celle de la naturalisation, du fait de la primauté donnée aux seules conséquences biologiques de situations qui relèvent avant tout de logiques sociales, économiques et politiques. De fait, ce modèle homogénéise une population en occultant la diversité et la complexité des trajectoires migratoires contemporaines.

Si les personnes migrantes, comme d'autres personnes issues de mondes sociaux stigmatisés ou disqualifiés, peuvent effectivement faire les frais d'un ethnocentrisme médical ou de discriminations, il arrive aussi que des dispositifs pensés comme spécifiques pour promouvoir l'accès aux soins aient les effets inverses de ceux qu'ils prétendent viser. Des dispositifs d'accès spécifiques pour les étrangers sans-papiers ont ainsi montré leur manque de pertinence sanitaire et économique, comme c'est le cas de l'Aide médicale d'État en France. Tandis que la régularisation pour soins peut assigner à une maladie-métier quand elle est le seul socle d'accès à des droits.

Le 29 avril 2014, l’Union européenne annonçait vouloir généraliser le dépistage des migrants. La sélection médicale aux frontières a fait partie des premières mesures adoptées par l'OMS en 1954. Cette continuité met en lumière l'ambivalence de la désignation d'une cible des politiques de santé dont il est parfois difficile de saisir ce qu'elle doit à une suspicion archaïque des sédentaires vis-à-vis des nomades, les premiers devant se protéger des dangers suscités par la mobilité des seconds. Il existe 
néanmoins des initiatives soucieuses des complexités et difficultés des trajectoires de l'exil, de l'expérience d'une condition minoritaire dans la société dite d'accueil: sans doute s'agit-il de les promouvoir. Mais il s'agit aussi d'être attentif aux écueils qui viennent d'être évoqués.

Ce sont les contraintes politiques, juridiques, économiques et sociales façonnant les mobilités contemporaines qui produisent de la vulnérabilité, laquelle n'est pas intrinsèque aux migrants et les affecte de manière non homogène. Cela témoigne d'un profond paradoxe du monde contemporain: la mondialisation est liée à une dynamique de disparition des frontières au sens de la circulation généralisée permise et, dans le même temps, génère un monde où l'obsession des frontières et le désir de murs semblent n'avoir jamais été aussi forts.

\section{Pour aller plus loin}

Agier, M. (2008). Gérer les indésirables. Des camps de réfugiés au gouvernement humanitaire. Paris, Flammarion.

Musso, S. et Sakoyan, J., Mulot et S. (2012). Migrations et circulations thérapeutiques: Odyssées et espaces. Introduction au dossier thématique En quête de soins: soignants et malades dans la globalisation. Anthropologie et Santé, http:// anthropologiesante.revues.org/1040

Withol de Wenden, C. (2009). La question migratoire au XXI siècle. Migrants, réfugiés et relations internationales. Presses de Sciences Po. 


\title{
31
}

\section{C'est parce qu'ils sont migrants qu'ils ont la tuberculose}

\author{
Marie-Ève Carle
}

À l'heure de la mondialisation, la multiplication des déplacements de populations (notamment par l'émigration) est fréquemment perçue comme un vecteur de maladies qui menacent la santé collective. On associe régulièrement les maladies infectieuses (SRAS, grippe $\mathrm{A}\left(\mathrm{H}_{1} \mathrm{~N}_{1}\right)$, $\mathrm{VIH}$, sida, etc.) à ce phénomène, car les individus viendraient de régions du monde où la prévalence de ces infections est plus importante. La tuberculose est l'une de ces maladies souvent associées au fait migratoire'. Qu'en est-il réellement?

Il importe de rappeler que malgré l'existence de traitements préventifs et curatifs, plus de 1,5 million de personnes meurent de la tuberculose annuellement, ce qui représente environ 5000 personnes par jour. Le nombre de cas varie énormément selon les régions du monde. L’incidence est considérable dans certaines régions de l'Afrique et de l'Asie qui représentent respectivement $31 \%$ et $55 \%$ des nouveaux cas de tuberculose à l'échelle mondiale. Quotidiennement, près de 25000 personnes contractent

1. La tuberculose est causée par une bactérie (le bacille de Koch) qui attaque le plus fréquemment les poumons. La transmission de la maladie est aérienne, au même titre que la grippe ou le rhume. En toussant, en crachant ou encore en éternuant, le malade projette des gouttelettes dans l'air qui peuvent être respirées par les gens de son entourage. Ces personnes courent alors le risque de devenir porteuses de la forme latente de la tuberculose (en opposition à la forme active). En période de latence, la maladie est asymptomatique et non contagieuse. 
cette maladie et deviennent porteuses de la forme latente. Ces personnes sont exposées à un risque variant entre $10 \%$ et $15 \%$ de développer la forme active de la maladie au cours de leur vie ${ }^{2}$. L'Organisation mondiale de la santé (OMS) signale qu'une personne sur trois dans le monde est porteuse de la forme latente de la tuberculose.

Bien que cette maladie soit toujours très présente dans les pays pauvres, on assiste à sa recrudescence dans les pays industrialisés, et plus particulièrement parmi les personnes les plus défavorisées. Certains groupes seraient donc plus vulnérables face à cette maladie, notamment les immigrants provenant de pays où la tuberculose est très présente.

Au Canada, les statistiques sont assez probantes à ce sujet. En trois décennies, le nombre de cas de tuberculose diagnostiqués chez des personnes nées à l'étranger a pratiquement doublé, ce qui représente actuellement plus de $66 \%$ des cas. La modification des pays d'émigration des individus venant s'installer au Canada est la principale explication de ce changement. Actuellement, la plupart des immigrants sont originaires de l'Asie et de l'Afrique, régions hautement affectées par la maladie. Or, dans les années 1960, l'émigration se faisait principalement de la France, de l'Angleterre et des États-Unis, qui avaient, à ce moment-là, des profils épidémiologiques similaires à celui du Canada. On observe aujourd'hui un nombre considérable de cas de tuberculose dans les grandes villes canadiennes (Montréal, Toronto, Edmonton, Vancouver, etc.) et plus particulièrement dans les quartiers dont la population se compose majoritairement d'immigrants.

Il importe de rappeler que la règlementation canadienne sur l'immigration impose un dépistage médical de la tuberculose. Les immigrants et les réfugiés atteints de la maladie n'obtiennent pas le droit d'entrer au Canada. Le dépistage concerne uniquement la forme active de la maladie et non pas la forme latente. Malgré tout, les immigrants comptent parmi les groupes où l'incidence de la maladie est la plus élevée. Pourquoi y a-t-il autant de cas de tuberculose au sein de ce groupe?

La forte prévalence de la tuberculose dans le pays d'origine de la quasi-totalité des immigrants représente le facteur le plus souvent répertorié afin d'expliquer ces taux élevés. Malgré tout, pour mieux comprendre

2. La tuberculose peut se développer à la suite d'une diminution des défenses immunitaires d'un individu. Plusieurs éléments peuvent expliquer cette diminution, tels que l'âge, la maladie, la sous-alimentation ou encore un traitement immunosuppresseur. 
le développement de la tuberculose chez ces immigrants, il faut se pencher sur les cinq premières années de leur vie dans le pays d'accueil. En effet, près de la moitié des cas diagnostiqués l'ont été au cours de cette période. Bien que nous nous intéressions plus particulièrement au Canada, ce constat est également observable dans plusieurs pays industrialisés, notamment les États-Unis et l'Australie. Il est possible de s'interroger sur les facteurs entraînant le passage de la forme latente à la forme active de la maladie chez les immigrants. Bien que plusieurs hypothèses concernant le stress, la génétique et l'affaiblissement du système immunitaire soient avancées, les conditions de vie jouent aussi un rôle important.

Tentons de comprendre cette vulnérabilité quant à la tuberculose chez les personnes en situation de migration. Les critères de sélection établis par les pays hôtes tendent à favoriser les jeunes en bonne santé. Par conséquent, les immigrants sont souvent en meilleure santé que la population locale à leur arrivée. Toutefois, cet avantage s'amenuiserait avec le temps pour rejoindre la moyenne nationale. Le même constat est observé en France et dans bon nombre de pays industrialisés. En lien avec cette diminution de l'état de santé, rappelons que tous les porteurs de la forme latente ne développent pas la forme active de la maladie. Il faut donc trouver des pistes d'explication à travers des facteurs biologiques, culturels, politiques et structurels. Si le nombre important de cas de tuberculose chez les immigrants est principalement expliqué par leur pays d'origine, le passage de la forme latente à la forme active s'explique plutôt par trois facteurs.

Plusieurs immigrants sont aux prises avec des conditions de vie déficientes dans les premières années suivant leur installation (pauvreté, logements surpeuplés, conditions précaires d'emploi, malnutrition, etc.), ce qui contribue au passage à la forme active de la maladie. Ces conditions dans les trajectoires post-migratoires introduisent des distinctions considérables quant aux probabilités de développer ou non la forme active de la maladie. Il devient alors possible d'envisager que le risque associé au développement de la tuberculose ne serait pas le même pour tous les immigrants selon leurs conditions de vie après leur installation.

L’impact négatif du stress sur la capacité du système immunitaire à se défendre est avancé par plusieurs auteurs afin d'expliquer la survenue de la maladie après l'établissement. Dix années sont parfois nécessaires à plusieurs immigrants pour accéder à une certaine stabilité financière. 
Plusieurs familles vivent sous le seuil de pauvreté pendant cette période. Cette hypothèse prend tout son sens et est intimement liée au stress pouvant être associé à des conditions de vie déficientes.

Laccès au système de santé demeure un élément primordial de la prise en charge de la tuberculose au sein de ce groupe. Toutefois, certaines études soulignent l'existence d'une certaine discrimination institutionnelle dont sont victimes les immigrants. Cela peut se présenter sous forme de perceptions stéréotypées de la part des professionnels de la santé, de barrières quant à la langue, de la méconnaissance du fonctionnement des institutions, etc. Tous ces éléments limiteraient l'utilisation des services et pourraient favoriser le développement de la maladie, faute de prévention.

Ainsi, l'idée reçue selon laquelle les immigrants apporteraient avec eux la tuberculose doit être largement nuancée en s'intéressant à leurs trajectoires d'établissement dans leur pays d'accueil. Les conditions de vie dans les années suivant leur installation influencent le développement ou non de la maladie et méritent de situer la réflexion dans un cadre beaucoup plus global sur l'impact des inégalités sociales de santé.

\section{Pour aller plus loin}

Organisation MONDiALE DE LA SANTÉ (OMS) (2015). Tuberculose. http://www.who. int/mediacentre/factsheets/fs104/fr/

Reitmanova, S. et Gustafson, D. L. (2012). Coloring the white plague: a syndemic approach to immigrant tuberculosis in Canada. Ethnicity \& Health, 17:4, 403-418.

TAPIÉRO, B. et CARLE, M.-È. (2009). Les maladies infectieuses: l'illusion du risque zéro. Montréal, Éditions du CHU Sainte-Justine. 


\section{LES SINGULARITÉS DES POPULATIONS}





\title{
L'obésité est un problème de riches dans les pays en développement
}

\author{
Hélène Delisle
}

L'obésité a rapidement pris des proportions épidémiques dans le monde. Reconnue maintenant comme une maladie chronique par l'Organisation mondiale de la santé (OMS), elle contribue à réduire l'espérance de vie et augmente le risque de diabète, de maladies cardiovasculaires et de plusieurs cancers. L'obésité est caractérisée par un excès de tissu adipeux. Elle est définie par un indice de masse corporelle (IMC) élevé (30 ou plus); le surpoids (IMC entre 25 et 30 ) est en quelque sorte son antichambre. Toutefois, c'est le gras qui se situe au niveau abdominal qui est le plus susceptible d'entraîner des problèmes de santé. On parle alors d'obésité abdominale, laquelle est définie par un tour de taille supérieur aux valeurs de référence pour le sexe. On doit donc distinguer obésité générale selon l'IMC et obésité abdominale selon le tour de taille. Les données populationnelles disponibles portent cependant essentiellement sur l'IMC.

Il y a une dizaine d'années, l'Afrique restait le seul continent où l'obésité ne représentait pas encore un problème de santé publique. Toutefois, les données fortement agrégées à l'échelle nationale occultent les profondes disparités au sein des pays. En 2008, la prévalence de l'obésité, alors de $10 \%$, avait plus que doublé en 15 ans en Afrique de l'Ouest urbaine, alors qu'elle restait faible et stable en milieu rural. Elle était trois fois plus fréquente chez les femmes que chez les hommes tant en ville qu'en milieu rural. L'obésité en Afrique subsaharienne affecte-t-elle donc essentiellement les citadins et, 
parmi eux, les mieux nantis, comme on le prétend encore souvent? L’augmentation rapportée reflèterait-elle simplement l'amélioration du niveau de vie, entre autres avec l'urbanisation? Qu'est-ce qui peut expliquer la prévalence incomparablement plus élevée d'obésité chez les femmes que chez les hommes, en Afrique de l'Ouest mais aussi en Afrique du Sud, alors que dans les pays industrialisés, c'est plutôt l'inverse?

L'analyse des données d'enquêtes démographiques et de santé (EDS) dans sept pays africains où deux enquêtes séparées par une décennie avaient été menées révélait qu'il y avait en moyenne $5 \%$ d'augmentation par an de la prévalence de surpoids ou d'obésité. Chez les femmes, il y avait une forte relation positive entre l'obésité et un niveau socioéconomique élevé. Mais en 10 ans, le surpoids/obésité avait progressé plus rapidement chez les femmes pauvres (+50\%) que chez les riches citadines $(+7 \%)$. En outre, il y avait une progression de $50 \%$ chez les femmes peu ou pas scolarisées, alors qu'on notait une diminution de $10 \%$ chez celles qui avaient un niveau secondaire ou supérieur. Ces données laissent à penser qu'avec le temps, les citadins pauvres seront autant, sinon plus, affectés par l'obésité que les citadins nantis. L'éducation pourrait plutôt faire reculer l'obésité chez les femmes. Elle pourrait notamment les amener à se détacher de l'image traditionnellement positive de l'obésité dans certaines sociétés.

S’agissant de la relation entre le niveau socioéconomique et l'obésité, relation qui n'est pas univoque, il est bon de distinguer non seulement le revenu des individus, mais également le niveau économique des pays. À la lumière de 244 EDS (878 ooo femmes) menées dans 56 pays entre 1991 et 2009, une étude montre que le surpoids chez les femmes était positivement associé à la résidence urbaine. Toutefois, l'association positive entre le niveau socioéconomique et le surpoids devenait plus faible à mesure que la richesse des pays augmentait. En outre, un niveau universitaire était négativement associé au surpoids, mais cette relation était modulée par le niveau de revenu des pays. Jusqu'à une richesse de 4000 \$US par habitant dans un pays, les femmes les plus éduquées étaient plus susceptibles d'être en surpoids que celles qui avaient moins fréquenté l'école. En revanche, au-delà de ce niveau de richesse du pays, ce sont les femmes les moins éduquées qui avaient la plus forte probabilité de surpoids. Ainsi, le risque d'obésité basculerait des plus riches aux plus pauvres et des plus éduquées aux moins éduquées avec le progrès économique des pays. Ce constat est congruent avec la plus forte prévalence d'obésité observée parmi les populations à faibles revenus dans les pays industrialisés. 
On avance souvent, pour tenter d'expliquer l'obésité de la pauvreté, qu'avoir une saine alimentation coûte plus cher, car ce sont les aliments denses en énergie mais relativement pauvres en nutriments qui coûtent le moins cher. Ceci a été démontré pour les pays industrialisés, mais aussi en Afrique du Sud, où a été menée la seule étude sur ce sujet dans un pays en développement.

Dans les pays à revenu faible ou intermédiaire, mais pas dans les pays à revenu élevé, il apparaît que l'obésité est plus présente en ville qu'en zone rurale. Dans une analyse de 28 études de populations en Afrique de l'Ouest, dont 13 réalisées en milieu urbain, des chercheurs ont montré que l'occidentalisation de l'alimentation, avec une consommation accrue de sucre et de gras, ainsi qu'un mode de vie sédentaire, pouvaient expliquer l'augmentation fulgurante de l'obésité en milieu urbain. Ces deux changements - alimentation plus dense en énergie et dépense physique moindre - caractérisent la transition nutritionnelle qui se produit à des rythmes variés dans les pays à faible revenu. Nos études ont montré que l'alimentation s'éloignait progressivement des habitudes traditionnelles en ville et que le schéma alimentaire urbain était associé à un risque accru de résistance à l'insuline (qui favorise le diabète). En outre, l'inactivité physique était un facteur important d'obésité et d'hypertension.

Une autre idée reçue est que l'obésité, ou tout excès de poids, est une surnutrition, diamétralement opposée à la dénutrition, qui serait son contraire. En réalité, l'obésité est aussi une forme de malnutrition, car coexistent souvent avec ce bilan calorique positif une ou des carences en d'autres micronutriments, comme le fer. En outre, les deux peuvent se cumuler, pour des populations et même des ménages. C'est ce qu'on appelle le double fardeau de la malnutrition. C'est ainsi qu'on observe assez fréquemment que les mêmes ménages abritent un adulte obèse ou en surpoids, souvent la mère, alors qu'un ou plusieurs enfants présentent un retard de croissance caractéristique d'une dénutrition chronique. En Afrique du Sud, pays à revenu intermédiaire où l'on observe une transition nutritionnelle rapide, le double fardeau de la malnutrition est fortement répandu, même en zone rurale. On rapportait récemment que le retard de croissance chez les enfants de moins de cinq ans coexistait avec un taux élevé d'obésité à l'adolescence, particulièrement chez les filles.

La surnutrition et la dénutrition présentent par ailleurs des liens étroits, puisqu'une dénutrition tôt dans la vie, c'est-à-dire pendant la vie intra-utérine ou l'enfance, augmentera par la suite les risques d'obésité et 
autres troubles cardio-métaboliques. Le fœtus qui aurait adapté son métabolisme à un environnement nutritionnel de manque serait très mal outillé pour faire face à une alimentation abondante combinée à la sédentarité. Il serait de ce fait prédisposé aux maladies chroniques métaboliques. Il y aurait ainsi une synergie entre les deux formes de malnutrition, la dénutrition favorisant plus tard l'obésité et les autres maladies chroniques associées. Cette situation devrait être une préoccupation importante pour l'Afrique subsaharienne, qui a connu ou connaît encore une forte prévalence de malnutrition maternelle et infantile.

L'obésité est certainement à prévenir et à contrer. S'agissant du surpoids, son incidence sur la santé est moins nette. D’après une analyse de près de 100 études, il n'y aurait pas d'excès de mortalité pour les individus qui sont en surpoids. Toutefois, un rapport ultérieur concluait, après une nouvelle analyse de ces mêmes résultats, que le surpoids augmente effectivement le risque de décès. Au demeurant, comme le surpoids conduit facilement à l'obésité, il importe de le prévenir également. L'Association internationale pour l'étude de l'obésité lançait tout récemment un plan d'action en 10 points à l'intention des gouvernements pour qu'ils contribuent à maîtriser l'obésité. Ce plan d'action mise sur le leadership des gouvernements qui, avec la société civile, pourraient prendre des mesures propices dans le marché, mais aussi dans d'autres secteurs.

Il apparaît que si l'obésité est pour l'instant proportionnellement plus fréquente parmi les couches privilégiées dans les pays à faible revenu d'Afrique, il est à craindre qu'elle augmente plus rapidement parmi les moins favorisées, particulièrement en milieu urbain et chez les femmes, accentuant encore davantage les inégalités socioéconomiques de santé.

\section{Pour aller plus loin}

Delisle, H. et Receveur, O. (2007). Les «dysnutritions" dans les pays en développement. CMAJ, 176:65.

Eberwine, D. (2003). La globésité: une crise aux proportions croissantes. Diabetes Voice, 48 (1) : 30-33.

Fontbonne, A., Cournil, A., Cames, C. et al. (2011). Caractéristiques anthropométriques et facteurs de risque cardio-métaboliques en milieu urbain au Sénégal. Médecine. Maladies métaboliques, 5 (3): 331-335. 


\title{
L'obésité concerne surtout les États-Unis d'Amérique
}

\author{
Laurence Guillaumie, Aissatou Coulibaly, Georges Batona, \\ Sophie Dupéré, Jacky Njdepel
}

On parle d'obésité lorsque l'indice de masse corporelle est supérieur ou égal à $30 \mathrm{~kg} / \mathrm{m}^{2}$, et de surpoids à partir d'un indice supérieur ou égal à $25 \mathrm{~kg} / \mathrm{m}^{2}$. Le surpoids et l'obésité ont des effets néfastes sur la pression artérielle, le cholestérol, l'insulino-résistance ou encore les articulations. Ils représentent donc des facteurs de risque pour les maladies du cœur, le diabète, les troubles musculo-squelettiques et certains cancers. Sur une population mondiale de sept milliards d'habitants, 2,8 millions meurent chaque année en raison d'obésité ou de surpoids, ce qui représente la cinquième cause de décès dans le monde.

Les États-Unis d'Amérique sont célèbres, non seulement pour leurs vedettes de cinéma, mais aussi pour leur taux d'obésité. En 2010, $36 \%$ des Américains de plus de 20 ans étaient obèses et $70 \%$ étaient en surpoids, en incluant l'obésité. Toutefois, ils ne sont pas les seuls. Beaucoup d'autres pays industrialisés sont eux aussi concernés. À titre d'exemple, le taux d'obésité est de $26 \%$ en Angleterre, de $25 \%$ en Australie, de $24 \%$ au Canada et de $18 \%$ en Irlande. Ce taux a progressé rapidement ces trente dernières années en raison de l'urbanisation, de la modification des habitudes alimentaires et de l'augmentation de la sédentarité des populations. Ces changements dans les modes de vie sont dus à des transformations majeures dans des secteurs tels que l'urbanisme et les milieux de vie, 
l'organisation du travail, les transports, l'agriculture, l'industrie agroalimentaire, la distribution, le marketing, etc. Dans ce contexte, les environnements dits obésogènes, c'est-à-dire qui conduisent à l'obésité, sont devenus la norme dans les pays industrialisés. Il serait toutefois inexact de conclure que ce constat s'applique également à tous les pays. En effet, certains pays industrialisés ont des taux de prévalence de l'obésité plus faibles, comme le Japon avec $4 \%$ ou la Suisse avec $8 \%$.

L’obésité n'est pas limitée aux États-Unis d'Amérique ou aux sociétés industrialisées; elle se retrouve aussi dans les pays à faible revenu qui présentent de plus en plus les caractéristiques des environnements obésogènes. On trouve de forts taux de prévalence de l'obésité dans toutes les régions du monde. En Amérique latine, le Mexique et le Venezuela rapportent un taux de $30 \%$ et le Chili, un taux de $22 \%$. Au Moyen-Orient, la prévalence de l'obésité rivalise avec celle des États-Unis. En Arabie Saoudite, le taux est de $28 \%$ pour les hommes et de $44 \%$ pour les femmes. Des résultats similaires ou un peu plus faibles sont obtenus au Koweït, au Qatar et en Jordanie. En Afrique, des taux élevés peuvent être constatés en Afrique du Sud, avec $20 \%$, ou en Égypte, avec $15 \%$. Cela contraste avec certains pays de l'Asie comme l'Inde, l'Indonésie ou la Chine, qui rapportent des taux de $2 \%$ à $3 \%$. Finalement, c'est dans le Pacifique et l'océan Indien que les prévalences d'obésité sont les plus élevées. Par exemple, des taux d'obésité supérieurs à $75 \%$ sont rapportés à Nauru, à Samoa, aux Samoa américaines, aux îles Cook, au Tonga et en Polynésie française. Certains pays à faible revenu, en plus de connaître la sousalimentation, peuvent donc être aussi touchés par l'obésité, en particulier dans les milieux urbains.

Certaines catégories de personnes ont été identifiées comme particulièrement vulnérables. Tout d'abord, dans tous les pays concernés par l'obésité, les enfants sont exposés à une alimentation riche en gras, en sucre, en sel, et pauvre en micronutriments. Cette alimentation, combinée à un faible niveau d'activité physique, se traduit par une forte augmentation de l'obésité infantile. Cette situation est préoccupante, car les enfants obèses sont susceptibles de le rester à l'âge adulte et de développer de façon plus précoce des maladies telles que le diabète et les maladies cardiovasculaires. Sur 43 millions d'enfants de moins de cinq ans en surpoids et obèses dans le monde, $80 \%$ vivent dans les pays à faible revenu. Aussi, on constate que les femmes sont particulièrement concernées par l'obésité. 
Le taux y est environ deux fois plus élevé que chez les hommes dans les pays à faible revenu, contrairement aux pays à revenus élevés, où le taux est similaire pour les deux sexes. En revanche, dans la plupart des pays à revenus élevés, les femmes peu scolarisées sont deux à trois fois plus susceptibles d'être obèses que les femmes plus instruites, alors qu'il n'existe peu ou pas de disparités pour les hommes. Si l'obésité a été un problème des pays à revenus élevés, il est aussi un problème mondial, touchant particulièrement les populations les plus pauvres.

Face à ces constats, l'Organisation mondiale de la santé (OMS) a tiré la sonnette d'alarme en décrétant une épidémie mondiale d'obésité, car cette dernière touche pratiquement tous les groupes d'âge, toutes les couches socioéconomiques et presque tous les pays. Puisque l'obésité est le plus souvent évitable, l'OMS invite les individus à limiter leur consommation de gras et de sucre, à augmenter leur consommation de fruits, de légumes, de légumineuses, de grains entiers et de noix. Il faut aussi s'engager dans une activité physique régulière, soit 60 minutes par jour pour les enfants et 150 minutes par semaine pour les adultes. L'OMS invite aussi les partenaires publics et privés à donner les moyens aux individus d'adopter un mode de vie sain, notamment en facilitant l'accès à l'activité physique régulière, à une alimentation saine, abordable et facilement accessible, et cela en particulier pour les personnes les plus pauvres.

\section{Pour aller plus loin}

Finucane, M. M. et al. (2011). Global Burden of Metabolic Risk Factors of Chronic Diseases Collaborating Group. National, regional, and global trends in body-mass index since 1980: systematic analysis of health examination surveys and epidemiological studies with 960 country-years and 9.1 million participants. Lancet, 377 (9765): 557-567.

OECD. (2010). Obesity and the Economics of Prevention: Fit Not Fat. OECD Publishing.

Ogden, C. L., Carroll, M. D., Kit, B.,et Flegal, K. M. (2012). Prevalence of obesity in the United States, 2009-2010. NCHS data brief (vol. 82). Hyattsville, MD: National Center for Health Statistics. 


\title{
Pour se soigner, les sociétés africaines sont solidaires
}

\author{
Anne Attané
}

La vivacité des solidarités au sein des familles africaines ne fait de doute pour personne. En effet, discutez à bâtons rompus en Afrique de l'Ouest ou en Europe au sujet de l'entraide et des solidarités et vous entendrez un discours bien argumenté (tant de la part d'Africains que d'Européens) qui décrira à loisir l'intensité des liens sociaux et du degré d'entraide dans les sociétés africaines. L'intensité de ces échanges est évoquée en opposition à l'individualisme forcené qui prévaut dans les relations interindividuelles en Occident, où règne le chacun pour soi. Ainsi, les solidarités africaines seraient si denses que l'on prête à ces sociétés le fait d'avoir créé de manière endogène un système de sécurité sociale. Certes, ce système de dons obligatoires entrave les possibilités de développement en contribuant à l'appauvrissement de personnes bénéficiant d'un revenu régulier qu'elles se voient dans l'obligation de redistribuer en partie. Ce même système, en imposant d'aider celui qui, dans la famille, est dans le besoin, sécuriserait chacun des membres de la famille et plus particulièrement tous ceux n'ayant accès à aucune ressource monétaire. Ainsi, nous aurions affaire à une organisation sociale centrée sur une forme de redistribution obligatoire entre les individus apparentés, ou liés par l'amitié, qui protègerait ces individus en leur permettant, par exemple, d'accéder aux soins, mais rendrait impossible tout développement économique réel. 
Qu'en est-il exactement? L'exemple de Victorine offre un début de réponse.

Victorine vient d'avoir 34 ans, elle vit dans un célibatorium ${ }^{1}$ de Lomé au Togo et travaille dans un hôtel/restaurant du bord de plage tenu par une Européenne, elle y fait le ménage. Le 2 avril 2014, alors qu'elle prenait un zem (moto taxi) pour se rendre à son travail, elle a eu un accident et s'est brûlée très gravement le mollet avec le pot d'échappement de la moto. Elle raconte: "Si j'étais allée à l'hôpital, j’aurais laissé trop d'argent là-bas!» Aussi, un cataplasme noir orne sa plaie: «C'est un médicament traditionnel», me ditelle. Cinq jours après l'accident, elle boite de plus en plus, tant la douleur est vive quand elle marche. Mais elle doit travailler, car chaque jour non travaillé est décompté sur son salaire de 30 ooo FCFA par mois ( 45 euros). Elle ne peut en aucun cas prendre le risque de la moindre dépense, tant son budget est déjà à peine suffisant pour couvrir ses besoins vitaux.

Victorine vit seule, loin de sa famille, et n'a pas d'enfant. Ses parents résident dans la région de Kpalimé à $120 \mathrm{~km}$ de là, ils sont agriculteurs. Victorine est la seule - elle a quatre frères et sœurs - à avoir migré en ville. L’un d'entre eux s'est installé aux États-Unis depuis plus de douze ans, mais personne n'a vraiment de nouvelles. Il téléphone à leurs parents restés au village et leur envoie un peu d'argent (100 ooo à 180 ooo FCFA, de 150 à 270 euros) tous les deux ou trois mois. Mais voici plus de six mois qu'ils n'ont aucune nouvelle de lui. Deux de ses sœurs sont mariées à Kpalime, l'une d'elles, institutrice, a deux enfants, son mari n'a pas d'emploi fixe, il se «débrouille»; elle-même «s'en sort» à peine avec son salaire. L'autre sœur de Victorine fait un petit commerce de bananes plantains au marché de Kpalime. Le cadet des frères cultive dans le champ de ses parents. Victorine est partie vers Lomé, pensant y trouver un emploi rémunéré et aussi un mari. Ses parents sont plutôt dans une position d'attente vis-à-vis d'elle et elle ne se sent pas autorisée à leur «demander quelque chose», c'est-à-dire à leur demander un quelconque soutien financier alors même qu'elle a souhaité pouvoir «voler de ses propres ailes»!

Ainsi, l'entraide intrafamiliale n'est pas systématique. Un ensemble de valeurs et de conditions président à sa mise en place effective. Les normes sociales rappellent que les relations entre les générations sont sous le sceau du régime de l'obligation et de la dette, dette que les enfants auraient contractée vis-à-vis de leurs parents et grands-parents pour avoir été élevés par eux. Aussi, le circuit préférentiel de l'aide doit normalement

1. Le célibatorium désigne la cour collective comptant plusieurs habitations qui regroupaient au départ des célibataires - d'où son nom - et qui offre la possibilité à des couples ou des familles de se loger à moindre coût en milieu urbain. 
s'opérer selon un sens précis. Il est du devoir d'un enfant devenu adulte d'entretenir ses parents lorsqu'ils deviennent plus âgés. L’anthropologue Claudia Roth parle d'un contrat générationnel implicite qui présiderait aux échanges entre les générations: «La règle du jeu s'énonce ainsi: les aînés, venus en premier, sont des créanciers, puisqu'ils ont élevé les plus jeunes. Ces derniers remboursent leur dette, en assurant la sécurité sociale des personnes âgées le moment venu.» (Roth, 2010: 98) Toutefois, ce contrat intergénérationnel se trouve contrarié dans sa réalisation par la précarité économique, l'augmentation de la pauvreté, la difficulté d'accès à un emploi rémunéré, mais aussi du fait des transformations des valeurs qui président à l'entraide. Des recherches anthropologiques menées auprès de personnes vivant avec le VIH témoignent du fait que ce sont parfois des personnes âgées, des hommes comme des femmes, qui prennent en charge leur enfant adulte infecté et parfois les enfants de ce dernier (Attané et Ouédraogo, 2011). Parfois aussi des enfants très jeunes sont le meilleur soutien de leurs parents malades.

Au-delà du simple contrat tacite qui régirait les relations entre les générations, dans les contextes ouest-africains, chaque membre de la famille a toujours participé d'une manière ou d'une autre, soit par son travail, soit en donnant son temps, soit en participant économiquement à la vie de la maisonnée. Un enfant, dès qu'il est en âge de se déplacer aisément et de parler clairement, est sollicité pour de multiples tâches. Ainsi, l'entraide est multidirectionnelle, mais des individus peuvent être à l'écart de tout recours possible. Par exemple, quand des jeunes enfants de 10 à 14 ans se révèlent être les seuls en position de soutien vis-à-vis de l'adulte malade, ceci témoigne davantage de l'isolement social, affectif et relationnel de cet adulte que de l'existence effective de l'entraide.

La monétarisation de la vie sociale comme l'augmentation du coût de la vie (soins, scolarisation des enfants, denrées alimentaires) ont amplifié la part des transferts monétaires au sein du groupe familial et créé une forme de marginalisation de celles et de ceux qui sont précisément à l'écart de ces transferts.

Ibrahim avait cinq ans et demi. Il vivait à Ouagadougou au Burkina Faso dans un quartier loti ${ }^{2}$ en grande famille auprès de son père et de sa mère ainsi que

2. La ville de Ouagadougou se décompose en zones dites loties et en zones non loties. Ces dernières sont celles où les constructions souvent en terre se sont faites sans aucune 
de son frère aîné âgé de neuf ans. Dans la cour familiale, une tante paternelle et des jeunes oncles paternels, son père et sa propre mère. Son grand-père paternel vit dans le quartier voisin. Ce dernier est le seul parmi tout l'entourage de l'enfant à bénéficier d'un emploi stable et d'un salaire régulier. Un matin, alors que la mère d'Ibrahim fait chauffer de l'eau dans une immense marmite en fonte, elle s'éloigne un instant, Ibrahim escalade une chaise en plastique proche de la marmite et tombe! Voyant qu'il est très gravement brûlé, sa mère le conduit à vélo dans le centre de santé du quartier où le personnel les dirige vers l'hôpital du quartier. Arrivés là, la mère et son enfant sont renvoyés vers l'hôpital national pédiatrique. La mère d'Ibrahim s'y rend à vélo, on lui demande d'acquitter la somme de 30 ooo FCFA (45 euros) afin que l'enfant soit soigné. Ne possédant pas cette somme d'argent, elle rentre à la maison avec son enfant et a recours à un traitement traditionnel. Après huit jours, le grand-père paternel est prévenu, le neuvième jour, il conduit l'enfant au centre hospitalier universitaire, ce dernier mourra le lendemain soir.

Ici, le système d'entraide est contrarié à plus d'un titre. Le père d'Ibrahim éprouve de la honte à l'idée de demander de l'aide à son propre père alors qu'il est lui-même père de famille. Il ne préviendra donc son père - le seul en mesure de payer - que très tardivement. La honte éprouvée par le père d'Ibrahim du fait d'être en incapacité économique de faire face aux besoins de son enfant se double d'une gêne liée aux difficultés qui président à la relation qu'il entretient avec son père. Le père d'Ibrahim vit dans la grande concession familiale du fait de sa précarité économique aux côtés de deux tantes paternelles divorcées. Le grand-père d'Ibrahim a, lui, pris son propre foyer dans lequel il vit avec sa jeune épouse villageoise moins âgée que son premier fils. Le père d'Ibrahim est donc au cœur de conflits familiaux entre des adultes qui, tous, pour des raisons spécifiques, vivent en position d'exclusion sociale ou économique. Le statut de femmes divorcées reste à Ouagadougou peu enviable, le nonaccès à un emploi rémunéré d'un père de famille fait de lui un homme faiblement considéré par son entourage. Ici, le déclassement social perçu par les intéressés a favorisé la survenue du drame: personne ne s'est vu en capacité économique de faire face aux soins ou de solliciter l'aide de celui qui était en mesure de le faire.

Parallèlement, il est des transferts de biens intrafamiliaux ou entre amis qu'il est très difficile d'appréhender, car ils restent tus. Par exemple,

autorisation de l'État et surtout sans que ces quartiers soient pourvus d'un accès à l'électricité, à l'eau potable ou au moindre circuit d'assainissement. 
comme le montre Fatoumata Ouattara dans ses travaux, une femme ne dira pas ouvertement que c'est elle qui a donné de l'argent à son époux pour assurer les frais de l'accouchement, de peur de ternir l'image de son mari. De même, un cadet peut être très fortement sollicité par son aîné pour participer au financement d'un événement particulier (un épisode de maladie d'un tiers), mais même si cette obligation incombe au chef de famille, il n'en dit rien. Reconnaître qu'on a donné de l'argent à un aîné pour assumer ce qui est sa charge signifie remettre ouvertement en cause ses capacités économiques et plus généralement ses capacités à assumer son rôle de chef de ménage. La figure de l'homme, chef de famille, pourvoyant aux besoins matériels de son épouse ou de ses épouses et à ceux de ses enfants, reste en effet très prégnante.

L'affection et l'attachement pour un tiers sont des motivations centrales de l'entraide. Le soutien se manifeste d'autant plus s'il y a un consensus de l'entourage sur les qualités et les mérites d'une personne. Parallèlement, exprimer son affection passe dans les contextes ouestafricains contemporains par un soutien moral et matériel, alors que le refus du don signe le rejet. L'entraide quotidienne n'est pas générale mais élective (Attané et Ouédraogo, 2008). Elle est soumise à l'incertitude et elle est fonction de la qualité de la relation et de son évolution, comme des conditions économiques d'existence de l'aidant. Ainsi, les personnes en manque ou rupture d'entraide sont nombreuses. L'histoire des relations intrafamiliales de chacun détermine les possibilités d'existence de l'aide. Ainsi, les actes effectifs de solidarité dépendent également de l'affection, de la reconnaissance, de la confiance, et parfois de la culpabilité qui préside à la relation.

À la lumière de ces deux récits, le manque d'argent et la pauvreté apparaissent comme les raisons premières entravant l'accès au traitement. Mais au-delà, les solidarités en Afrique de l'Ouest ne s'exercent pas de manière systématique. L’entraide n'est pas généralisée, elle est plutôt élective: un individu qui peut aider décide d'accorder son aide à un frère ou une sœur plutôt qu'à un autre. La motivation dépend, certes, de l'ampleur et de la gravité des difficultés éprouvées par celui ou celle qui a besoin d'aide, mais elle est tout autant motivée par des valeurs morales que l'aidant prête à celui ou celle qu'il décide de soutenir. Quelqu'un considéré comme vertueux, dynamique, travailleur, méritant recevra plus facilement de l'aide que celui ou celle auquel ou à laquelle on accorde peu de mérite 
personnel. Ainsi, les membres d'un même groupe familial ne sont pas mis à l'abri de manière identique et comparable. Certains d'entre eux sont complètement exclus du système d'aide.

\section{Pour aller plus loin}

Attané, A. et OuÉdraogo, R. (2011). Lutter au quotidien: effets de genre et de génération sur l'entraide intrafamiliale en contexte de VIH au Burkina Faso. In A. Desclaux, P. Msellati et K. Sow (dir.), Femmes et VIH dans les pays du Sud, Paris, Editions de l'ANRS.

Rотн, C. (2010). Les relations intergénérationnelles sous pression au Burkina Faso. Autrepart, Vieillir au Sud, $\mathrm{n}^{\circ} 53$ : 95-110.

VUARIN, R. (2000). Un système africain de protection sociale au temps de la mondialisation. Ou «venez m'aider à tuer mon lion». Paris, L'Harmattan. 


\section{Les Africaines \\ font beaucoup d'enfants}

Solène Lardoux

La croissance de la population en Afrique au sud du Sahara est la plus forte au monde (2,5\% par an), en particulier parce que le début de la transition démographique est récent et que les niveaux élevés de fécondité passés et actuels ont des effets à long terme (5,1 enfants par femme en 2005-2010).

Toutefois, les variations de la fécondité sont diverses, car les taux sont soit en baisse, en stagnation, ou plus rarement en hausse. Des différences importantes de niveaux de fécondité apparaissent entre milieux urbain et rural ainsi qu'entre les capitales de différentes régions. Mais à l'inverse, il peut exister une certaine homogénéité régionale. La fécondité est généralement la plus basse dans la capitale et parmi les femmes les plus instruites. Par exemple, en 2006 au Kenya, l'ISF (indice synthétique de fécondité) était de 5,9 enfants par femme en milieu urbain (où vit $24 \%$ de la population) comparé à 6,5 en milieu rural. Au Mali, $35 \%$ de la population vit en milieu urbain, où la fécondité s'élève à 5,4 dans l'ensemble urbain et à 4,8 enfants par femme dans la capitale de Bamako seulement. Cependant, en milieu rural, où vit la grande majorité de la population, l'ISF est de 7,2 enfants par femme. Par contraste, au Tchad et au Niger, où un très faible pourcentage de la population vit en ville ( $2 \%$ et $18 \%$ respectivement), les différences de fécondité entre les deux milieux de résidence sont faibles, seulement d'environ un enfant par femme. 
Les déclins les plus rapides de la fécondité ont eu lieu en Afrique australe, à tous les âges et dans les générations successives (Afrique du Sud, Zimbabwe, Botswana, passant de 6 à 2,5 enfants par femme entre 1960-1965 et 2005-2010), au Ghana et au Kenya (de 7-8 enfants par femme à 4-5 entre 1960-1965 et 2005-2010), à Maurice, au Cap-Vert et à La Réunion (de 6-7 enfants par femme à 2 entre 1960-1965 et 2005-2010). Par contraste, dans plusieurs pays d'Afrique centrale et de l'Ouest, la transition de la fécondité est à peine commencée. Les nombres d'enfants par femme sont les plus élevés au Mali, en Guinée, au Tchad (6,8 à 7 enfants par femme) et le maximum est observé au Niger (7,6 enfants par femme en moyenne).

Ainsi, l'affirmation selon laquelle "les femmes africaines font beaucoup d'enfants» mérite d'être nuancée. Outre la présentation précédente de chiffres, qui montre une diversité de situations, nous exposerons trois dimensions liées à la régulation de la fécondité sur ce continent, soit la demande en matière de contraception, le recours à l'avortement et les pratiques matrimoniales et sexuelles.

Des recherches ont montré que les femmes utilisaient la contraception pour arrêter et espacer les naissances en fonction du nombre d'enfants déjà nés et de l'âge du plus jeune enfant. La volonté de retarder les naissances est de plus en plus associée à l'utilisation de la contraception. Par conséquent, le report de la fécondité devrait avoir des implications importantes sur les niveaux de fécondité et sur la croissance de la population en Afrique.

Cependant, l'analyse des résultats d'enquêtes quantitatives rend compte des cas où les femmes ont des besoins non satisfaits de contraception, puisqu'elles déclarent vouloir retarder leur prochaine naissance ou arrêter d'avoir des enfants, mais elles n'utilisent pas de moyen de contraception moderne. En Afrique de l'Est et australe, l'utilisation des contraceptifs avait augmenté et était associée à une baisse de ces besoins non satisfaits, alors qu'en Afrique de l'Ouest et centrale, ces derniers restaient supérieurs à l'utilisation de contraceptifs. Des facteurs limitant l'accès peuvent expliquer ces dernières situations, tels qu'une trop grande distance géographique avec les points de distribution de contraceptifs, le coût des produits, les effets secondaires sur la santé des femmes, ou encore un approvisionnement irrégulier et insuffisant des méthodes contraceptives. D'autres freins correspondent aux restrictions imposées par les professionnels de la santé à cause notamment d'un manque de formation 
adéquate sur les contraceptifs adaptés aux besoins des patientes, ou encore au fait que le processus pour la prescription et la délivrance d'un contraceptif est trop complexe et inadapté, et que les professionnels de santé font intervenir des normes et valeurs sociales associées au statut matrimonial, à l'âge des patientes ou au nombre d'enfants qu'elles ont déjà eus, dans leur décision de prescrire un contraceptif. Les méthodes contraceptives utilisées en Afrique subsaharienne sont principalement des méthodes non définitives (pilule, DIU, Ogino, etc.). Le recours à la stérilisation féminine ou à la vasectomie masculine est très rare, sauf en Afrique australe ou au Kenya.

En plus des contraceptifs, la baisse de la fécondité parmi les jeunes générations est liée à l'augmentation de la fréquence du recours à l'avortement, mais celui-ci a souvent lieu dans des conditions précaires, car clandestines. Dans plusieurs cas, ce dernier est seulement autorisé pour sauver la vie d'une femme lorsque sa santé mentale ou physique est en danger ou qu'elle a été victime d'un viol ou d'inceste (Swaziland, Bénin, Togo, Éthiopie, Guinée, Mali). Au Tchad et au Niger, l'avortement est autorisé seulement pour protéger la santé de la mère, mais exclut les cas d'inceste ou de viol. L'existence de ces lois restrictives et la condamnation populaire répandue de la pratique de l'avortement ont pour conséquence que la plupart des avortements sont pratiqués dans des conditions risquées. Dans les cas de viol et de violence sexuelle, d'une part, les femmes préfèrent ne pas recourir à un avortement légal, ou n'en ont pas les moyens et, d'autre part, ni les professionnels de la santé ni la police ne connaissent bien les conditions et les procédures à suivre lorsque l'avortement devrait être légal. Une remise en cause du système légal semble nécessaire, puisque seules les femmes les plus riches connaîtront un médecin qui pourra les assister lors d'un avortement, sans tenir compte de la reconnaissance de sa légalité.

Notre troisième point concerne un élément du statut matrimonial qu'est la polygamie. La polygamie dans une société peut contribuer à une fécondité élevée, car elle tend à maximiser le temps passé par les femmes dans le mariage (au sein des sociétés où le mariage a lieu à un âge relativement jeune et où le remariage est fréquent après un divorce ou un veuvage), et donc au fait d'avoir des enfants. Par ailleurs, les hommes continuent d'avoir des enfants après l'âge de 60 ans. Pour ce qui est des femmes, la polygamie peut être associée à une plus faible fécondité indi- 
viduelle des femmes mariées en union polygame que celles vivant en union monogame pour plusieurs raisons. Tout d'abord, en union polygame, la fréquence des rapports sexuels pour chacune des épouses est plus faible qu'en union monogame. Selon l'hypothèse de favoritisme, la femme favorite, souvent la plus jeune, aurait une fécondité plus élevée que les autres coépouses. Quand les coépouses d'un homme polygame habitent dans la même concession de maisons avec leur mari, l'abstinence sexuelle pendant la période d'allaitement peut être plus longtemps observée grâce à l'alternance des relations sexuelles entre les partenaires, ce qui allonge les intervalles entre les naissances pour une femme. Deuxièmement, la fécondité plus basse des femmes en union polygame peut aussi s'expliquer par un effet de sélection, car un mari peut prendre des épouses supplémentaires lorsqu'il considère qu'il n'a pas eu tous les enfants qu'il désirait avec sa première femme. Les femmes sans enfant sont surreprésentées dans les unions polygames. Enfin, dans la polygamie, en contraste avec la monogamie, les époux ont moins de chances de vivre ensemble et ainsi les femmes sont moins exposées au risque de grossesse.

Ainsi, la fécondité élevée des femmes africaines est liée aux valeurs et pratiques traditionnelles et pronatalistes, mais aussi aux inégalités entre hommes et femmes, entre générations, et aux contraintes liées aux freins sanitaires et sociaux à l'utilisation de la contraception. En effet, les femmes africaines font beaucoup d'enfants, mais leurs intentions de fécondité ont baissé et les écarts entre les intentions de fécondité et la fécondité réalisée à la fin de la vie reproductive restent grands dans certaines régions de l'Afrique subsaharienne.

\section{Pour aller plus loin}

Lardoux, S. et van de Walle, É. (2003). Polygamie et fécondité en milieu rural sénégalais. Population, vol. 58, $\mathrm{n}^{\circ}$ 6: 807-836.

N'Bouké, A., CALvès, A. et LARDoux, S. (2012). Le recours à l'avortement provoqué à Lomé (Togo): Évolution et rôle dans la réduction de la fécondité. Population, vol. $67, \mathrm{n}^{\circ} 2: 357-385$.

Tabutin, D. et Schoumaker, B. (2004). La démographie de l'Afrique au sud du Sahara des années 1950 aux années 2000. Synthèse des changements et bilan statistique. Population, vol. 59, $\mathrm{n}^{\circ}$ 3-4: 519-621. 


\title{
L'homosexualité n'existe pas en Afrique
}

\author{
Michel Bourrelly
}

Depuis quelques années, dans de nombreux pays africains jusqu'alors muets sur le sujet, des mouvements radicaux de lutte contre l'homosexualité ont vu le jour. Les raisons mises en avant par ces mouvements sont de plusieurs ordres: religieux, moral ou traditionnel. «L'homosexualité n'est pas africaine» est tout de même ce que l'on entend le plus, certains allant même jusqu'à dire que "c'est une maladie de Blancs». En Afrique, au-delà des mouvements intégristes religieux bien campés sur des positions dogmatiques, les populations sont enclines à considérer que l'homosexualité n'est qu’une «déviance européenne».

Sur les continents asiatique, américain et européen, depuis des siècles, souvent à mots couverts, parfois de façon stigmatisante, l'homosexualité est décrite, pratiquée, décriée ou évoquée. Sur le continent africain, la tradition est orale, la majorité des références à la pratique d'une sexualité entre des personnes d'un même sexe met en avant son origine forcément étrangère. Toutefois, on peut trouver des traces d'observations concrètes, telles que les décrit Daniel Vangroenweghe. C’est ainsi que dès 1558, João Dos Santos parle d'hommes passifs, appelés Chibudi au bas Congo et au nord de l'Angola. Dès 1616, alors que le mariage pour tous n'était à l'ordre du jour nulle part sur le globe, Petrus Jarris parlait de deux prêtres qui, en Angola, ont vu des hommes vêtus comme des femmes qui se mariaient. Tout au long des siècles qui ont suivi, les mêmes observations, agrémentées 
de propos réprobateurs, ont été imprimées et publiées sur l'Afrique et les Africains. Le professeur Cheikh Ibrahima Niang, anthropologue sénégalais, plaide pour la dépénalisation des délits de prostitution et d'homosexualité. En effet, il affirme que l'homosexualité n'est pas un «fait occidental», «cette pratique a existé depuis l'Antiquité, depuis l'Égypte pharaonique. Ces sociétés ont pu gérer ce phénomène». Certes, "toutes les religions l'interdisent, mais la foi est un domaine privé», tranche-t-il.

La maladie importée des «Blancs» et héritée de la colonisation est l'homophobie. La plupart des articles de loi pénalisant l'homosexualité sont directement tirés des codes français et anglais de l'époque coloniale.

Pour sa part, le sida a été sans aucun doute l'activateur de cette homophobie, car l'infection à VIH à ses débuts a été fortement marquée par les contaminations en milieux homosexuels. La reconnaissance d'une épidémie importante en Afrique n'était pas pensable pour les dirigeants africains. De plus, un certain nombre d'acteurs de la société civile ne pouvaient imaginer la possibilité d'associer le terme Africain avec celui d'homosexuel. Dès lors, bien que conscients de l'existence de pratiques homosexuelles, tout aussi nombreuses qu'ailleurs dans le monde, mais certes différentes par de nombreux aspects, de savants subterfuges furent employés pour nier leur existence en Afrique. Certains sont allés jusqu’à faire disparaître le terme d'homosexuel et le substituer par des acronymes obscurs. Pour autant, cela ne fit pas disparaître les homosexuels, ni les contaminations par le VIH découlant des actions de prévention influencées par des normes hétérosexuelles qui, on le sait, diminuent lorsque des messages adaptés et délivrés par les pairs sont diffusés. Les États décidèrent de renforcer l'application des législations jusqu'alors somnolentes et de livrer à la vindicte populaire les homosexuels en les rendant responsables de tous les maux.

L'homosexualité n'est pas une maladie, et elle n'est pas cantonnée hors du continent africain. Les nouvelles techniques de communication ont permis à tous ceux qui se sentaient différents par leurs attirances pour des personnes du même sexe de savoir qu'ils (et elles) n'étaient pas seuls, et qu'ils n'étaient pas des déviants et encore moins des anormaux. 
184 - DES IDÉES REÇUES EN SANTÉ MONDIALE

\section{Pour aller plus loin}

Bourrelly, M. (2009). Urgence pour les hommes ayant des rapports homosexuels http://vih.org/200910o1/afrique-urgence-pour-hommes-ayantrapports-homosexuels-7303

NiANG, C. I. (2013). Anthropologie de la sexualité: philosophie, culture et construction sociale du sexe au Sénégal. Thèse d'État.

Vangroenweghe, D. (200o). Sida et sexualité en Afrique. Editions Aden. 


\title{
C'est à cause de leur culture que certains patients ne suivent pas leur traitement
}

\author{
Sylvie Fortin
}

On a longtemps reproché à la médecine son absence de prise en compte de la culture dans le soin. Depuis, nous avons un peu versé dans son contraire, notamment avec un appel à la compétence culturelle. Mais de quelle culture s'agit-il? Celle du migrant de l'Ouest ou du Sud, américain ou haïtien, scolarisé ou moins instruit, portant le hijab coloré ou noir? Nos milieux cosmopolites d'ici et d'ailleurs, la migration et la mondialisation des échanges participent à la déconstruction de cultures pensées comme homogènes, aux frontières définies, partagées par un groupe donné. Entendue comme un ensemble variable de valeurs, de croyances, de modes d'être prenant sens dans un contexte relationnel et historique, la culture fluctue et est mobilisée différemment selon les situations. La culture du professionnel de la santé, comme celle du patient et de sa famille, est en devenir. Elle n'existe pas seule, en dehors des contextes qui la façonnent.

En milieux hospitaliers, on réclame souvent d'expliciter un phénomène dit culturel dès lors que le patient ou sa famille sont perçus comme autres. Cette mise à distance n'est pas étrangère aux rapports sociaux qui prévalent dans la localité circonscrite. L'autre sera migrant (statut réel ou imaginé) et plus spécifiquement minoritaire dans le contexte local, de par son groupe d'appartenance (ethnique, confessionnel) ou encore ses marqueurs phénotypiques et sociologiques (dont la langue, mais aussi la tenue, 
la couleur de la peau, les pratiques). Cette mise à distance est aussi activée lorsqu'il y a une hésitation à suivre les directives thérapeutiques proposées par les équipes de soins. À son tour, ce refus des projets thérapeutiques est fréquemment attribué à un différend culturel, religieux ou de genre.

Laltérité se dessine en fonction de soi qui, dans l'espace hospitalier, évoque une certaine idée de la science ou même de la raison pour le personnel soignant. Lorsque le patient et sa famille tendent à trop diverger par rapport à ce qui est acceptable comme attitude à adopter dans l'espace thérapeutique, ils sont identifiés comme à problème. Corollairement, l'expression d'une bonne compréhension de la condition médicale du patient, du langage médical et une attitude, un comportement estimé adéquat dans la situation sont des atouts pour le patient et sa famille, pour la perception qu'en aura l'équipe soignante et les relations qui s'ensuivront. Or, ces ressources que nous qualifions de symboliques sont inégalement partagées, en milieu hospitalier comme ailleurs. En voici un exemple:

\section{Histoire de Tahina'}

Une fillette d'âge préscolaire fréquente le même milieu hospitalier depuis sa naissance. Sa trajectoire de soins est remplie d'embûches: incertitude quant au diagnostic, délais dans les interventions, complications imprévues, résistance aux médicaments, etc. La relation de Tahina avec le personnel soignant est excellente. La dynamique entre les parents et le personnel soignant est néanmoins problématique. Certains épisodes sont caractérisés par des conflits engendrés par une résistance, voire un refus, de ses parents aux traitements proposés par l'équipe soignante. Une mauvaise représentation et compréhension de la maladie ainsi que des limitations intellectuelles des parents sont évoquées par certains soignants pour expliquer ces tensions. Les parents seraient incapables de comprendre les interventions proposées, ce qui expliquerait leur refus des soins pour leur fille. Les parents sont d'un groupe minoritaire peu favorisé dans la localité, leur situation économique est difficile, notamment en raison de la longue maladie de Tahina et de leur présence soutenue à son chevet. Leur compétence linguistique (langue locale) est limitée, mais fonctionnelle, sans qu'ils en comprennent nécessairement toutes les subtilités (jeux de mots, par exemple).

Selon les codes (les cadres de référence) de l'unité de soins concernée, le bon parent est proactif, s'informe, est à l'affût de symptômes qui pourraient

1. L'histoire intégrale de Tahina (nom fictif) a été publiée dans Fortin (2013). 
renseigner l'équipe soignante sur l'évolution de l'état de l'enfant. Or, l'implication des parents de Tahina dans la trajectoire de soins ne correspond pas à celle attendue. Leurs hésitations quant au plan de traitement deviennent un obstacle, un frein au projet thérapeutique. Un bon parent ne refuserait pas le traitement proposé.

Devant le refus d'acceptation du traitement par le père, un interprète est appelé afin de lui expliquer le bien-fondé de l'intervention proposée. Par la suite, un autre père (du même pays d'origine), ayant jadis approuvé des traitements similaires, est présenté aux parents, afin de mieux les informer. Les parents de Tahina sont rassurés par les paroles de cet autre père qui, à sa manière, élucide le langage médical. Le père de Tahina est légitimé dans cet échange. Ses questions et hésitations sont entendues par l'autre père alors qu'elles étaient écartées par plusieurs soignants. Enfin, le père en vient à approuver les traitements. Pour les cliniciens, cet épisode est qualifié de problème culturel parce qu'il s'agit d'une famille d'origine immigrante dont les perceptions du corps et des soins (réelles ou imaginées) diffèrent des perceptions locales.

Comme tant d'autres, cette histoire raconte cette rencontre souvent difficile entre différents types de savoirs (experts et profanes), mais aussi, celle entre migrants et non-migrants et plus encore, entre majoritaires et minoritaires, dans l'espace clinique. La complexité des trajectoires thérapeutiques et les différentes temporalités de la maladie (les familles et les professionnels de la santé ne perçoivent souvent pas la situation de la même manière), les conditions structurelles d'hospitalisation et l'organisation des services, les différentes capacités d'expression de langue publique, les ressources symboliques et, dans cette histoire, les conditions de vie précaires de la famille participent aussi à cette dynamique.

Cette histoire étaye aussi notre propos quant au fait que l'insistance sur la culture dans le soin est un leurre, un voile qui peut brouiller l'examen de la trajectoire de soins afin de mieux saisir les éléments qui entravent ou favorisent l'établissement d'une relation de confiance entre les professionnels de la santé, les patients et leur famille. Certes, la culture traverse nos vies, nos milieux, teinte notre compréhension, inspire nos pratiques. Elle colore notre expérience de la maladie, la nôtre et celle de nos proches. Elle n'en est pas moins mouvante, fluide, ancrée dans la proximité et traversée par le lointain. Elle résiste à tout enfermement et à toute catégorisation, notamment celle d'un groupe ethnique ou national partageant des traits culturels communs. La compétence culturelle participe à cette impression qu'un savoir balisé peut ordonner la différence. 
Nous retenons plutôt la notion d'humilité culturelle et la curiosité qu'elle sous-tend, l'invitation à une pratique ouverte à la diversité qui est peutêtre, en elle-même, le prérequis à toute rencontre clinique. Le philosophe Paul Ricœur situe la connaissance sur cette voie, avec comme première étape la "reconnaissance de la méconnaissance». La clinique n’y échappe pas. L'incertitude est inhérente à la diversité. Reste à l'apprivoiser. C'est sans aucun doute un défi central à la pratique soignante en milieux cosmopolites, où la diversité ethnique, religieuse et sociale rend caduque toute typologie territoire, groupe, culture.

Par-delà la culture, l'hôpital est un lieu de contact privilégié entre migrants et non-migrants et de manière inclusive, entre minoritaires et majoritaires. À titre d'institution phare de la société locale, l'hôpital joue un rôle qui dépasse sa vocation de soins; il est un acteur dans la ville. C’est un lieu apolitique, car les soignants qui y travaillent le font en fonction de leurs statuts professionnels et non de leurs points de vue personnels sur le social, la migration ou la diversité religieuse. Et comme le disait si bien un clinicien: «Ce n'est pas de moi dont il s'agit mais de mon patient» (notre traduction). En même temps, l'hôpital est un espace politique, puisqu'il engage des relations entre groupes, entre acteurs, entre savoirs, entre valeurs et normes. Au-delà des questions médicales, l'hôpital a aussi une portée sur la vie des patients et de leur famille. Cette institution représente un lien avec la société locale et le mainstream pour de nombreuses familles d'immigration récente et moins récente. Envisagé ainsi, l'hôpital réinvente sa vocation d'accueil et d'hospitalité au cœur de son projet initial, amalgamé à des savoirs de pointe qui constituent les fleurons de la médecine hospitalière contemporaine.

\section{Pour aller plus loin}

Cognet, M. et Montgomery, C. (dir.) (2007). Éthique de l'altérité. La question de la culture dans le champ de la santé et des services sociaux. Ste-Foy, Les Presses de l'Université Laval.

DelVecchio-Good, M.-J., Willen, S. S., Hannah, S. D., Vickery, K. et Taeseng PARK, L. (dir.) (2011). Shattering Culture. New York, Russel Sage Foundation.

Fortin, S. (2013). Conflits et reconnaissance dans l'espace social de la clinique. Les pratiques hospitalières en contexte pluraliste. Anthropologie et sociétés, 37(3): 179-200. 


\section{Les hommes qui ont des relations sexuelles avec d'autres hommes sont largement responsables de la transmission du VIH}

David Lessard, Bertrand Lebouché et Réjean Thomas

Depuis l'apparition de la pandémie de VIH/sida en 1981, les hommes ayant des relations sexuelles avec d'autres hommes ( $\mathrm{HSH}$ ) ont été les premières victimes de cette maladie. Ils restent touchés de manière disproportionnée par le virus de l'immunodéficience humaine (VIH). Trente ans après la découverte du virus, l'incidence élevée du VIH parmi les HSH est recensée sur tous les continents. Ainsi, les Caraïbes, avec $25 \%$ des HSH vivant avec le VIH, est la région où ils sont les plus touchés. En Afrique subsaharienne, où l'ensemble de la population est la plus éprouvée par le VIH, la prévalence parmi les $\mathrm{HSH}$ atteint $18 \%$, contre $5 \%$ dans la population adulte générale. Dans l'ensemble de l'Amérique du Nord, $15 \%$ des HSH vivent avec le VIH. On retrouve une proportion équivalente de HSH dans les grandes villes occidentales. Alors que l'épidémie globale est maintenant en déclin ou stabilisée, le VIH connaît une nouvelle résurgence parmi les $\mathrm{HSH}$, malgré les multiples campagnes de prévention destinées à cette population et la mise massive sous thérapie antirétrovirale des personnes vivant avec le VIH. En effet, une thérapie efficace contre le VIH réduit de plus de $95 \%$ le risque de transmission du virus d'une personne séropositive à son ou ses partenaires sexuels. 
Pour les HSH, la prévention de la transmission du VIH s'est surtout concentrée sur les risques individuels d'exposition à ce virus: relation anale sans préservatif; nombre élevé de partenaires masculins; utilisation concomitante de drogues ou d'alcool; et quantité importante de VIH dans le sang et dans le sperme qui, souvent, vient lui-même d'être infecté dans un contexte de multipartenariat.

Pour réduire les facteurs de risque, les campagnes de prévention du VIH ont mis l'accent sur le changement des comportements sexuels. Durant les années 1980 et 1990, elles ont valorisé différentes mesures comme l'abstinence, la réduction du nombre de partenaires, la fidélité sexuelle ou l'usage du condom. La notion de responsabilité individuelle s'est peu à peu implantée dans les discours et pratiques de prévention. En conséquence, les HSH sont souvent jugés responsables de la prévalence élevée du VIH parmi eux et cela est souvent invoqué pour justifier des stéréotypes défavorables et discriminants à leur égard. Selon ces stéréotypes, les HSH ne se protègent pas suffisamment contre le risque de transmission du VIH; ils gardent ainsi un risque élevé d'être infectés et de transmettre le VIH, et cela, malgré l'information qu'ils ont reçue. Les comportements jugés à risque ont été associés à la transmission du virus, entraînant du coup la stigmatisation, voire la criminalisation des personnes. Étant donné que l'incidence du VIH parmi les HSH ne diminue pas, ces derniers sont perçus comme à risque, ou incapables de changer leurs comportements, sinon irresponsables et immoraux.

Toutefois, les données scientifiques montrent que la transmission du VIH chez les HSH est liée à des facteurs bien plus complexes que leur simple responsabilité individuelle. Des facteurs biologiques, épidémiologiques et sociaux expliquent mieux leur forte vulnérabilité.

Parmi les facteurs biologiques, une relation sexuelle anale non protégée est la pratique sexuelle la plus à risque pour acquérir le VIH, en particulier pour le partenaire réceptif (celui qui est pénétré). La composition et la fragilité de la muqueuse anale la rendent plus perméable au virus. Le risque de transmission du virus lors d'une relation anale est 18 fois plus grand que lors d'une relation vaginale. On estime même que si le risque de transmission anale du VIH était le même que le risque vaginal, on diminuerait de $98 \%$ les nouveaux cas de VIH chez les HSH.

Dans les couples hétérosexuels, le partenaire homme pénètre et la partenaire femme est pénétrée. Le changement de rôle est beaucoup plus 
fréquent chez les HSH. Les relations anales non protégées entre HSH exposent les partenaires réceptifs, qui peuvent ensuite transmettre le virus à d'autres partenaires lorsqu'ils sont insertifs (lorsqu'ils pénètrent leur partenaire).

De plus, un nombre important d'HSH prend part à des relations anales réceptives et insertives, ce qui n'est pas le cas dans la population hétérosexuelle, où les rôles sont en quelque sorte prédéfinis par le sexe biologique. Si cette aptitude à jouer les deux rôles sexuels n'existait pas, on pourrait réduire les nouveaux cas dans cette population de $20 \%$ à $50 \%$.

D’un point de vue épidémiologique, dans un contexte où plus de $15 \%$ de la population HSH est touchée par le VIH, le risque pour un $\mathrm{HSH}$ séronégatif d'avoir un partenaire sexuel séropositif est plus important que dans un contexte hétérosexuel. De plus, le fait que les HSH aient plus fréquemment des partenaires occasionnels qu'un seul partenaire stable sur le long terme est également un moteur important de l'épidémie chez les HSH. Ainsi, si les rapports anaux non protégés intervenaient avec des partenaires stables et non occasionnels, les nouveaux cas de VIH chuteraient de $30 \%$ à $50 \%$. Ces données permettent de rendre compte des environnements différents dans lesquels évoluent les HSH. Pour avoir un impact réel chez les HSH, le préservatif doit être utilisé systématiquement, avec tous les partenaires. Une telle utilisation n'est pas possible dans la vie de tous les jours et sur toute la durée de la vie sexuelle, pour l'ensemble des HSH. Parfois, le préservatif peut être perçu comme une barrière entre les deux partenaires qui cherchent au contraire à approfondir leur intimité. D’autres fois, il n'est pas toujours disponible ou utilisé dans des conditions optimales. De plus, la consommation d'alcool et de drogues avant ou au cours de l'acte sexuel peut altérer le jugement et réduire l'utilisation des condoms.

Sur le plan social, l'homophobie et la discrimination envers les HSH contribuent à rendre ces derniers plus vulnérables, autant dans les pays reconnaissant certains principes d'équité pour les HSH que dans les 80 États du monde où les relations homosexuelles sont criminalisées. La discrimination et l'homophobie sont vécues de différentes manières selon les contextes. Par exemple, les autorités des prisons de plusieurs pays africains refusent de distribuer des condoms aux détenus sous prétexte que les relations sexuelles entre hommes y sont criminalisées. Pourtant, le VIH y est responsable de plus de la moitié des morts chez les détenus. 
Dans les pays industrialisés, les HSH migrent vers les centres urbains ou fréquentent des endroits spécifiques pour rencontrer des partenaires sexuels et se créer de nouveaux réseaux. Dans les grandes villes, ces réseaux sont parfois denses et avec de nombreux contacts sexuels entre les membres de ce réseau, exposant ainsi leurs membres à différentes infections et de manière plus fréquente. La configuration de ces réseaux et le fait que les HSH ont en moyenne plus de partenaires sexuels que d'autres groupes, accélèrent la transmission du VIH,

De plus, l'accès aux services de santé par les HSH varie énormément d'une région à l'autre et selon le contexte socioéconomique. En raison de l'homophobie intériorisée ou provenant de leur entourage, certains HSH hésiteront à parler de leur sexualité et à demander de l'information médicale. Dans certains contextes, ils se verront refuser l'accès aux soins de santé. Cette situation a des implications directes pour les campagnes de prévention émergentes qui combinent éducation, dépistage et traitement du VIH. L'accès aux soins de santé passe d'abord par l'accès au dépistage du VIH et des autres infections transmissibles sexuellement et par le sang. Le dépistage doit devenir une routine chez les HSH sexuellement actifs. Il doit être accessible dans leurs endroits de socialisation. Le test rapide avec résultat immédiat devrait être privilégié et offert par d'autres HSH formés à cette technique. De nouvelles stratégies d'autotests utilisables chez soi pouvant être utilisées, en particulier, comme dépistage avec son partenaire avant une relation sexuelle, sont en développement. L'accès aux soins de santé renvoie ensuite à l'utilisation massive des traitements contre le VIH dès le diagnostic. Ces traitements sont maintenant considérés comme l'un des moyens de prévention les plus efficaces. Un individu séropositif adhérant à une thérapie antirétrovirale peut réduire la quantité de virus dans son sang et ses liquides corporels jusqu'à atteindre un niveau indétectable. Le risque de transmission secondaire à ses partenaires est alors réduit de plus de $90 \%$, même sans une utilisation systématique du condom. Les thérapies antirétrovirales jouent donc un rôle fondamental et démontré dans la prévention de la transmission du VIH aux partenaires sexuels.

En résumé, réussir une prévention efficace du VIH chez les HSH nécessite de dépasser une simple responsabilité individuelle pour s'orienter vers une responsabilité davantage collective. On peut fonder de grands espoirs dans de nouvelles stratégies combinant une culture du dépistage 
fréquent (au moins une fois par an pour les HSH), une mise sous traitement rapide à la suite d'un dépistage positif et un soutien efficace pour le maintien du traitement à long terme. Les HSH séronégatifs devraient être informés et avoir accès aux nouvelles stratégies biomédicales de prévention. La prévention s'organise aussi au sein des communautés locales et globales, par des actions en amont et en aval de l'infection au VIH et culturellement adaptées. Il s'agit de s'attaquer efficacement aux facteurs structurels qui rendent les HSH particulièrement vulnérables au VIH comme l'homophobie, la stigmatisation et la discrimination.

\section{Pour aller plus loin}

Beyrer, C., et al. (2012). Global Epidemiology of HIV infection in men who have sex with men. The Lancet, july: 19-29.

Center for Control Disease (2014). HIV Among Gay, Bisexual, and Other Men Who Have Sex With Men, Fact Sheet. http://www.cdc.gov/hiv/risk/gender/msm/ facts/index.html 


\title{
La participation des femmes au microcrédit explique la violence conjugale en Asie du Sud
}

\author{
Katia Mohindra
}

Le microcrédit, une intervention pour lutter contre la pauvreté qui s'est répandue dans les pays à revenu faible et intermédiaire, vise à offrir aux pauvres l'accès au crédit pour améliorer leurs chances de mener des activités rémunératrices. En Asie du Sud, le mouvement du microcrédit s'est concentré plus spécifiquement sur les femmes, puisqu'il serait un moyen d'augmenter l'accès de ces dernières aux ressources, d'augmenter leur participation dans les décisions relatives au ménage et d'améliorer l'efficacité des programmes de microcrédit, puisque les femmes sont plus susceptibles d'adhérer aux règles des programmes et de rembourser les prêts en temps opportun.

Un nombre important de critiques au sujet du microcrédit ont été émises dont, notamment, le fait que cette intervention n'atteint pas les plus pauvres, le risque que le recours à des prêts se fasse uniquement à des fins de consommation et la convoitise des maris suscitée par les prêts accordés à leurs femmes. Cependant, la critique la plus dommageable demeure celle ayant trait à la violence émise à l'égard des femmes participant à un programme de microcrédit. On a avancé que la participation à un programme de microcrédit peut provoquer de la violence chez les hommes puisque, d'une part, les relations de pouvoir au sein du ménage sont perturbées par une plus grande autonomie sociale et financière de la 
femme et, d'autre part, l'accès des femmes au microcrédit constituerait un moyen pour le mari de contrôler l'objectif et l'utilité du prêt accordé, notamment en imaginant convoiter chez une femme une dot plus importante. Cette idée s'est introduite dans la conscience de nombreux spécialistes du développement et chez les féministes.

La violence contre les femmes est de plus en plus considérée comme un problème majeur de santé publique, un problème social aussi bien qu'une violation grave des droits de l'homme. Les médias et les politiciens portent davantage attention à cette violence, comme en témoigne la sollicitude importante et les vives réactions locales à la suite du viol et du meurtre brutal d'une jeune femme par un gang dans un bus de Delhi en 2012. Cet incident tragique a incité, à son tour, les tribunaux indiens à adopter une législation plus sévère contre le viol.

Cependant, relativement peu d'attention est accordée aux formes moins spectaculaires, mais plus répandues de violence envers les femmes en Asie du Sud, dont notamment la violence conjugale. Alors que cette dernière est présente au sein de diverses sociétés, elle est particulièrement répandue en Asie du Sud (selon certaines estimations, elle toucherait $50 \%$ des ménages), où les normes patriarcales sont particulièrement fortes et la violence envers les femmes étroitement liée au mariage. Elle survient souvent au début du mariage et se veut un signe de l'autorité de l'époux dans la relation maritale. Dans certaines régions du Bangladesh et de l'Inde, elle est utilisée pour extorquer plus d'argent de la dot ou pour mettre la main sur les biens de sa femme ou de la famille de son épouse.

Il semble donc nécessaire d'aborder la thématique de la violence conjugale en Asie du Sud en vue d'améliorer la santé des femmes, leur statut et leurs droits. Pour l'appréhender adéquatement, nous avons besoin d'une compréhension accrue de ses déterminants. L'idée préconçue que le microcrédit engendre de la violence conjugale et, par conséquent, représente une menace pour la santé des femmes, vient porter atteinte à l'image du microcrédit et peut nuire aux interventions visant précisément à réduire la violence conjugale.

La clarté semble faire défaut dans les discussions à ce sujet. Il est nécessaire d'aborder cette question précise en partant des données probantes disponibles.

Peu de recherches systématiques ont étudié le lien entre la participation des femmes au microcrédit et la violence conjugale en Asie du Sud. Les 
quelques données existantes ne sont pas basées sur des méthodes reconnues comme rigoureuses pour démontrer un lien de cause à effet. Par conséquent, il n'existe aucune donnée probante qui démontre que le microcrédit engendre bien de la violence conjugale.

Les données sur le lien entre la violence conjugale et la participation des femmes au microcrédit en Asie du Sud sont basées sur des études transversales et qualitatives. Les résultats de ces études ont été contradictoires. Certains auteurs ont constaté que les femmes qui participent au programme de microcrédit sont plus susceptibles d'être violentées, alors que l'inverse a été constaté par d'autres auteurs. Ces différences peuvent s'expliquer par les méthodes divergentes des études. Elles peuvent aussi s'expliquer par la relation complexe, non linéaire, entre l'autonomie de la femme et la violence de son mari. Le fait d'améliorer l'éducation, les compétences et le statut financier a été démontré comme un facteur de développement du statut et des capacités des femmes. Cela peut aussi conduire à une plus grande violence envers les femmes, car elles commencent à exprimer leur opinion à leurs maris, gagnent un revenu (qui peut être plus élevé que le leur), apparaissent plus souvent en public, ou font toute autre chose qui vient contester les normes et les rôles traditionnels. Il est également possible que les femmes qui participent au microcrédit aient un risque accru de faire face à la violence quand elles rejoignent le programme, puisque les maris doivent s'adapter aux nouveaux rôles et activités de leurs épouses. Mais ce risque tend à se transformer en un facteur protecteur de la violence conjugale, lorsqu'un certain seuil a été atteint, dans lequel les avantages de la participation de la femme l'emportent sur la dynamique du changement des genres, où le microcrédit devient une intervention socialement plus acceptable pour les femmes de la communauté.

En somme, il est possible que la participation des femmes au microcrédit accentues certains facteurs de la violence conjugale (et c'est peutêtre seulement temporairement) pour certaines d'entre elles. Toutefois, dans ce cas, le coupable n'est pas le programme du microcrédit en soi, mais plutôt le bouleversement des relations entre les genres généré par l'autonomie de la femme. La solution n'est pas de restreindre les possibilités pour les femmes de bénéficier d'un crédit et de s'engager dans des activités rémunératrices, mais plutôt d'améliorer la conception du microcrédit et d'intégrer des activités complémentaires dans le cadre du 
programme (par exemple, un programme de formation participative sur la violence conjugale a été intégré en Afrique du Sud) ou de mettre en œuvre des politiques et des interventions spécifiques qui répondent à cette problématique.

La participation des femmes au microcrédit ne fournit-elle pas tout simplement à certains hommes une raison pour s'en prendre à elles? Cette forme de violence est largement tolérée dans de nombreuses régions d'Asie du Sud, notamment dans certaines circonstances pour lesquelles les maris ont, apparemment, le droit de battre leurs épouses. Si c'est effectivement le cas, la solution n'est pas en lien avec le microcrédit, mais plutôt dans la remise en cause de ce supposé droit des maris de recourir à la violence en toute occasion.

\section{Pour aller plus loin}

BATES, L. et al. (2004). Socioeconomic factors and processes associated with domestic violence in rural Bangladesh. International Family Planning Perspectives, 30 : 190-199.

Mohindra, K. S. (2009). Women's Health and Poverty Alleviation in India. New Delhi: Academic Foundation.

Pronyk, P. et al. (2006). Effect of a structural intervention for the prevention of intimate-partner violence and HIV in rural South Africa: a cluster randomised trial. The Lancet, 368: 1973-1983. 


\title{
Les femmes sont responsables de leur assassinat par leur partenaire
}

\author{
Marie France Labrecque
}

En 2013, l'OMS publiait un rapport montrant que $30 \%$ des femmes à l'échelle mondiale subiraient de la violence de la part de leur partenaire intime. Les conséquences sur la santé des femmes sont nombreuses et le rapport montre qu'à l'échelle mondiale, $38 \%$ des femmes assassinées l'ont été par leur partenaire intime. C'est l'Afrique qui arrive en tête des régions du monde avec $45 \%$, alors que les Amériques et les pays à revenu élevé arrivent respectivement aux quatrième et cinquième rangs avec $36 \%$ et $33 \%$. Le rapport évoque que, très souvent, c'est la femme elle-même qui est blâmée pour ce qu'elle subit, même lorsque la violence entraîne sa mort.

Cette propension à penser que la victime - en l'occurrence la femme - est coupable de ce qui lui arrive est extrêmement répandue et seule la rectitude politique empêche certaines catégories de personnes de l'affirmer. Pour lui faire écho, on retrouve l'idée selon laquelle les causes de la violence sont à rechercher dans la psychologie masculine, soit que les hommes ne peuvent contrôler leurs pulsions ou qu'ils font des crises de folie. Cette idée est transversale à toutes les classes sociales. En 2013, alors que la Cour suprême du Canada entamait l'examen de certaines lois limitant l'exercice de la prostitution, un brillant et respecté avocat affirmait à la télévision que la prostitution correspondait à «des besoins 
ici au Canada ${ }^{1} »$. Certes, il n'a pas parlé de pulsions, mais on peut se demander quels seraient donc ces «besoins», au juste?

Peu de temps auparavant, lors d'une interview à la radio sur le cas des féminicides à Ciudad Juárez ${ }^{2}$ pendant laquelle j'expliquais que les quelque 1500 femmes assassinées entre 1993 et 2013 dans cette ville frontalière du Mexique, après avoir été violées, mutilées et même torturées, l'avaient été principalement en raison du système d'impunité généralisée régnant dans cette ville, l'animateur persistait à m'interroger sur les raisons psychologiques poussant les hommes à perpétrer ces crimes. Que l'on pense encore en 2013 que la prostitution correspond à un besoin ou que des assassinats massifs de femmes sont causés par la maladie mentale est révoltant. Mais, réflexion faite, cela n'est pas surprenant. Ce sont là des idées reçues qui ont la vie longue, très longue. Dans les deux cas, elles sont liées à la configuration même de la société patriarcale, et plus particulièrement à l'idéologie patriarcale, qui fait des hommes des protagonistes et des femmes des réceptacles passifs.

L'idéologie patriarcale situe la valeur des femmes dans la reproduction et la maternité, en plus de s'appuyer sur des systèmes binaires d'opposition (l'obligation à l'hétérosexualité est l'un de ces systèmes) qui, à leur tour, renvoient à la distinction entre le privé et le public. Alors que les femmes courent davantage de risques d'essuyer de la violence au sein même de leur foyer que n'importe où ailleurs, c'est en vertu de cette idéologie binaire qu'elles sont blâmées pour celle qu'elles subissent lorsqu'elles en sortent. Elles n'avaient qu'à rester chez elles, dans la sphère privée! Ainsi, lorsque la femme passe de la sphère privée pour investir celle du public, elle devient, toutes proportions gardées, une femme publique, trop facilement associée à la figure de la prostituée. Un peu à la façon de cette dernière, elle transgresse les frontières de la société, mais alors que la prostituée répond à un «besoin», celle-ci agresse les hommes, ou du moins, elle joue avec le feu. Et, une fois le processus enclenché, le fardeau de la preuve reposera sur elle. On se rappellera sans doute le film The Accused (1988) dans lequel l'actrice Jodie Foster personnifie une jeune femme violée par trois hommes dans

1. En ligne: http://www.radio-canada.ca/nouvelles/societe/2013/06/13/oo1prostitution-cour-supreme-audience.shtml. Voir particulièrement le propos tenu à la minute $5: 43$.

2. L'interview portait sur la publication de mon livre Féminicides et impunité. Le cas de Ciudad Juárez. Montréal: Écosociétés, 2012. 
un bar. L'affaire est portée devant la justice mais, au départ, les chances de la jeune personne d'obtenir la condamnation des violeurs sont considérées comme nulles parce que ces derniers estimaient qu'ils avaient été «provoqués» par la façon dont elle était habillée et par son comportement. L'avocate avait gagné cette cause, car elle avait pu prouver que la jeune femme avait dit «non» au moment des viols.

Dans la vie réelle, il y a peu de chances d'obtenir justice dans un cas comme celui-là, malgré toutes les avancées dans le domaine des droits humains des femmes, et plusieurs d'entre elles, si bien évidemment elles survivent, préfèrent continuer à se taire après avoir subi de la violence et même le viol. Se confiner dans la sphère privée n'est pourtant pas une solution pour les femmes. En effet, alors que la maison (espace privé) est considérée, dans la mouvance de l'idéologie patriarcale, comme la place idéale des femmes, elle est dans les faits un des lieux les plus dangereux pour elles, comme le montrent les taux élevés de violence intime sur tous les continents.

Tenir les victimes pour responsables de leur sort, s'agissant de la violence à l'encontre des femmes, ou encore justifier la prostitution par le besoin qu'elle contribuerait à combler, équivaut à tomber dans le piège même de l'idéologie patriarcale. Pour réfuter ces idées et les combattre, les enquêtes et les statistiques comme celles effectuées et fournies par l'OMS sont certes utiles, mais il importe également de sortir du cadre de cette idéologie et d'élargir la perspective aux causes structurelles. La violence des partenaires intimes à l'encontre des femmes ne peut être vue comme un simple geste individuel qui serait dicté, surtout ou seulement, par un dérèglement psychologique. Il s'agit du dernier maillon d'une chaîne de violences que l'on peut suivre de proche en proche, jusqu'au niveau international, et qui touche à la fois les femmes et les hommes. Le fait que ces derniers soient ceux qui majoritairement tuent les femmes relève de processus à long terme de construction de la masculinité, laquelle à son tour doit être mise en relation avec des dynamiques économiques, politiques et idéologiques du système mondial dans lequel nous vivons. Le même type de raisonnement peut s'appliquer à la prostitution, qui est loin de relever seulement de la décision individuelle d'une femme en particulier. Plus que jamais, dans le contexte du capitalisme néolibéral, la prostitution à la petite journée est elle aussi le dernier maillon d'une chaîne qui implique des hommes de toutes catégories, y compris de grands 
patrons de corporations privées ou publiques qui, désormais, dans le contexte d'une marchandisation de tous les aspects de la vie, ont le loisir de choisir les femmes correspondant à leurs critères à partir de catalogues en ligne.

Pour combattre les idées reçues qui servent d'ancrage à ces pratiques, il faudra bien davantage que des constats, des groupes de conscientisation ou des refuges, mais certainement, rien de moins, qu'un changement de société, ici ou ailleurs.

\section{Pour aller plus loin}

Sur le patriarcat: la revue Nouvelles questions féministes.

Sur les liens entre genre, patriarcat et système économique mondial: R. W. ConNelL (2002). Gender. Cambridge: Polity Press.

Sur les liens entre prostitution et marché: S. Hawthorne (2004). Wild Politics: Beyond Globalization. Women's Studies International Forum 27 : 243-259. 


\title{
L'excision est un rite initiatique pratiqué en Afrique, chez les musulmans et dans les villages
}

\author{
Fabienne Richard et Khadidiatou Diallo
}

L'Organisation mondiale de la santé (OMS) définit les mutilations sexuelles féminines comme «toutes les interventions aboutissant à une ablation partielle ou totale des organes génitaux externes de la femme ou toute autre lésion des organes génitaux féminins pratiquée à des fins non thérapeutiques». On parle d'excision quand le clitoris et les petites lèvres ont été coupés, et d'infibulation quand les grandes lèvres ont été cousues ensemble en ne laissant qu'un tout petit orifice pour l'écoulement des urines et du sang menstruel. Il a fallu attendre 1997 et des mouvements de femmes en Afrique et en Europe pour que ces pratiques soient reconnues comme une mutilation par l'OMS. Malgré une médiatisation de la question après la publication de livres biographiques, tels que Fleur $d u$ désert de la célèbre mannequin somalienne Waris Dirie (livre ensuite adapté au cinéma) et des activités de sensibilisation - le 6 février de chaque année reconnu comme journée internationale contre l'excision -, beaucoup d'idées reçues circulent autour de la pratique de l'excision. Ainsi, beaucoup de personnes pensent que l'excision n'est pratiquée que par des musulmans, en Afrique noire, et que cela touche les populations rurales et illettrées. La réalité de la pratique est peu connue et on entend 
qu'il s'agit d'une pratique traditionnelle d'un autre âge en voie de disparition (des missionnaires ont en effet fait des rapports de type ethnographique décrivant avec détail des cérémonies d'excision) et que là où elle se pratique, elle contribue à l'initiation de la jeune fille (passage de l'enfance vers le statut de femme).

\section{Un rite initiatique}

Beaucoup justifient le maintien de l'excision comme rite initiatique, celui du passage de l'enfance à l'âge adulte, du moment où une jeune fille deviendrait femme. Pourtant, ce qu'on observe est plutôt un abaissement de l'âge de l'excision. Au Mali, $77 \%$ des filles sont excisées avant leur cinquième anniversaire. Au Yémen, la majorité des enfants sont excisés dans leurs premières semaines de vie. Devient-on femme à trois mois de vie? À trois ans? Plus qu'un rite initiatique, l'excision est un rite identitaire. On le fait pour appartenir au groupe, pour ne pas être différent du reste du groupe. On excise pour se conformer à une norme sociale. Pour la communauté internationale, l'excision est reconnue comme une violation de l'intégrité physique et mentale des filles et des femmes. C'est une violation des droits humains.

\section{Une pratique en voie de disparition}

Il est arrivé plusieurs fois que des intervenants, et parmi eux des gens travaillant dans la coopération au développement, nous lancent à propos de la pratique de l'excision: "Ah, mais c'est du passé, cela ne se fait plus... » $\mathrm{Si}$, dans certains pays, on peut noter une baisse de la prévalence de l'excision dans les tranches d'âge des 15-19 ans, comme la Côte d'Ivoire ou le Kenya, dans d'autres pays, on ne voit quasiment aucune avancée. C'est le cas de la Somalie, de la Guinée, de la Gambie. Selon le dernier rapport de l'UNICEF de 2013, on estime à plus de 125 millions le nombre de femmes déjà excisées dans le monde, et à 30 millions de nouveaux cas dans les dix prochaines années. Il faudra encore plusieurs générations avant que la pratique soit complètement abolie. 


\section{Elle n'existe qu'en Afrique}

C'est une des idées reçues les plus fréquentes, car on ne présente souvent que la carte de l'Afrique lorsqu'on parle de l'excision. Même l'UNICEF fait cette erreur dans son dernier rapport qui dresse un état des lieux de la pratique (paru en 2013). On devrait présenter une carte du monde pour s'apercevoir que d'autres pays et d'autres communautés sont concernées. Par exemple, on excise en Indonésie, en Inde, en Malaisie. On excise aussi en Irak (Kurdistan, nord de l'Irak, où la prévalence est de $44 \%$ ). On excise également en Europe, comme en témoignent les nombreux procès qui ont eu lieu en France dès les années 1990.

Quand on évoque l'Afrique, les gens pensent directement à l'Afrique noire, l'Afrique subsaharienne. C'est toujours une surprise quand on rappelle que l'Égypte est l'un des pays les plus touchés du continent avec une prévalence de plus de $90 \%$. Les conséquences peuvent être dramatiques. Ainsi, en 2007 et en 2013, les médias ont relayé la mort de jeunes filles en Égypte à la suite d'excisions médicalisées. Dans ce pays, plus des trois quarts des excisions sont pratiquées par des professionnels de santé malgré la condamnation ferme de l'OMS de la médicalisation de mutilations sexuelles.

\section{Seuls les musulmans la pratiquent}

Beaucoup de personnes pensent que l'excision n'est pratiquée que par des musulmans. Or, selon les historiens, la pratique aurait démarré en HauteÉgypte du temps des pharaons, donc bien avant les religions monothéistes et l'écriture de la Bible et du Coran. En Sierra Leone, $80 \%$ des femmes chrétiennes étaient excisées en 2008. Inversement, on observe que plusieurs pays musulmans du continent africain ne pratiquent pas l'excision, comme l'Algérie ou la Tunisie. Il s'agit au départ d'une pratique qui est plus déterminée par le groupe social que par la religion. Il faut cependant reconnaître que certains chefs religieux musulmans font leur propre relecture du Coran et prônent l'excision au nom de la religion. Mais d'autres la dénoncent, comme Muhammad Sayyid Tantawi, qui, à la tête de la mosquée égyptienne Al-Azhar, a déclaré en 2006 qu'il ne retrouvait aucun passage dans le Coran qui indiquait que les filles devaient être excisées. On ne retrouve pas ce genre de prêche pour l'excision chez les 
prêtres catholiques, ce qui pourrait expliquer la diminution plus rapide de la pratique ces dernières années parmi les communautés chrétiennes, comme c'est le cas par exemple au Mali. En 2006, dans ce pays, $40 \%$ des femmes de religion chrétienne déclaraient ne pas vouloir exciser leur fille contre $9 \%$ chez les femmes de religion musulmane.

Contrairement à l'Afrique, où on retrouve l'excision dans les différentes religions, en Asie, elle est pratiquée principalement par des communautés musulmanes. C'est le cas de l'Indonésie, de la Malaisie.

\section{On ne la voit que dans les villages}

Une idée reçue classique suppose que l'excision serait pratiquée en milieu rural. Elle n'existerait pas en milieu urbain, où les gens auraient un niveau socioéconomique et un niveau d'étude plus élevés. Là encore, comme pour la religion, il n'y a pas de frontières. Les familles pratiquent l'excision en ville, la seule différence sera peut-être qu'en ville, la mère emmènera son enfant dans un cabinet médical. Ainsi, en Guinée en 2012, la prévalence de l'excision est la même en milieu urbain et rural (97\%).

Même si l'éducation des filles est en soi une étape vers l'émancipation des femmes, elle ne résout pas tout, si l'approche genre n'est pas enseignée en classe. On verra donc des femmes universitaires faire exciser leur enfant pour respecter la tradition. L'excision est une violence de genre qui est tellement intégrée dans certaines sociétés qu'elle est devenue une norme sociale. On dit même d'une femme excisée qu'elle est complète. C'est cette prise de conscience par les femmes de leurs droits sexuels et reproductifs qui fera changer les choses et un dialogue au sein du couple. La dernière étude de l'UNICEF montre une discordance importante entre les opinions de l'homme et de la femme au sein des ménages par rapport à la poursuite de la pratique. Chacun croit que l'autre est en faveur de l'excision et explique son accord. Ainsi, les femmes disent vouloir exciser leur fille parce que les hommes le demandent, alors que ces mêmes hommes sont plus nombreux à s'opposer à la pratique et disent que c'est une histoire de femmes. Il faut donc en parler, rendre les débats sur l'excision publics via les médias (radio, télé, internet) pour qu’un vrai dialogue s'instaure au sein des familles.

Nous avons donc vu que la pratique de l'excision est encore bien ancrée dans beaucoup de pays, qu'elle touche parfois plus de $90 \%$ de la 
population féminine, qu'elle n'a pas de religion, de classe sociale et qu'elle s'étend bien au-delà de l'Afrique subsaharienne, au Moyen-Orient et en Asie. Aujourd'hui, l'excision prend de nouvelles formes, on coupe les filles de plus en plus tôt sans avertissement, ni apprentissage ou initiation autour de l'acte. On coupe pour marquer les filles dans leur corps et réduire leur plaisir sexuel. Du point de vue des droits humains, cette pratique est le reflet d'une inégalité entre les sexes profondément enracinée, et constitue une forme extrême de discrimination à l'encontre des femmes.

\section{Pour aller plus loin}

Bellas-Cabane, C. (2008). La coupure. L'excision ou les identités douloureuses. Éditions La Dispute.

Études démographiques et de santé avec leur module «excision» : www.dhsprogram. com

UNICEF (2013). Mutilations génitales féminines/excision: aperçu statistique et étude de la dynamique des changements. www.unicef.org/wcaro/french/4494_7461.html 


\title{
Avec tous leurs privilèges, les communautés autochtones devraient pouvoir prendre en charge leur santé
}

\author{
Faisca Richer
}

Il subsiste au sein de la population québécoise une grande méconnaissance de l'histoire des populations autochtones. Par conséquent, nombreux sont les mythes qui persistent sur les enjeux qui les concernent. Parmi les idées reçues les plus répandues, la croyance que les Autochtones sont des citoyens privilégiés bénéficiant de droits supérieurs aux autres citoyens est toujours présente au Québec.

Ainsi, dans les tribunes des grands journaux, on voit de façon routinière les commentaires suivants:

"Je pensais qu'on était au XIX ${ }^{e}$ siècle pas au XVIII. Les authoctones $(s i c)$ n'ont-ils pas les mêmes privilèges que les Blancs? N'ont-ils pas droit à d'autres privilèges qui ne sont réservés qu'à eux? En tant que descendante des Français qui ont colonisés (sic) le pays, je ne vois vraiment pas ce qui m'attendrirait davantage sur leur sort que sur le mien. [...] 300 ans m'apparaissent suffisant pour sortir du bois.

«[...] il faut en revenir des lamentations stériles et, [...] il faut se prendre en main.»

Certes, les Québécois n'ignorent plus que les Autochtones furent, à travers l'histoire, victimes de traumatismes collectifs graves. Plusieurs savent aussi que ces populations sont affectées par des problèmes sociaux 
et de santé (dépendances, violence, suicide, etc.). Toutefois, peu de Québécois connaissent les véritables raisons de cet état de santé désastreux, et plus rares encore sont ceux qui comprennent qu'il est en fait une conséquence directe des traumatismes historiques vécus.

Or, pour bien interpréter la situation des populations autochtones au Québec, il convient de rétablir les faits sur deux questions fondamentales. En premier lieu, les populations autochtones sont-elles aussi privilégiées qu'on le prétend? Et en second lieu, ces privilèges constituent-ils véritablement ce dont une société a besoin pour "se prendre en charge» et améliorer la santé de ses membres? Pour illustrer notre propos, nous emploierons l'exemple des communautés des Premières Nations vivant au Québec ${ }^{1}$.

\section{Les Premières Nations, des citoyens privilégiés?}

Certes, les membres des Premières Nations ne disposent pas des mêmes droits que les autres citoyens canadiens. En fait, leurs droits sont définis par la Loi sur les Indiens qui, depuis 1876, établit un régime de tutelle des populations. En effet, selon les dispositions de cette loi, le statut des «bénéficiaires» équivaut à celui d'un enfant mineur soumis au contrôle de son tuteur légal (le gouvernement fédéral) pour tous les aspects de sa vie. C'est donc en vertu de cette loi que le gouvernement définit qui est «indien", à quel territoire il a droit, et quelle est la structure politique de sa communauté. Malgré de multiples ajustements apportés jusqu’en 1985, la Loi sur les Indiens est toujours en vigueur. C'est évidemment à tort qu'elle est considérée comme un régime de privilèges.

En réalité, cette législation prive les membres des Premières Nations qui y sont assujettis de certains droits fondamentaux, tels que celui d'accès à la propriété. En vertu de la Loi, les terrains dans les réserves appartiennent au gouvernement; ils ne peuvent donc être ni achetés, ni vendus, ni faire l'objet d'un prêt. De même, selon la Loi, les biens des Premières Nations vivant dans une réserve ne sont pas saisissables; l'accès au crédit à la consommation s'avère donc souvent impossible, et ce, quels que soient le revenu et la solvabilité du demandeur. Conséquemment, sans véritable

1. Les détails de la situation des Inuit et des Métis sont différents, mais les grands constats demeurent analogues. 
accès au crédit ou aux prêts hypothécaires, comment se surprendre que rares soient les membres des Premières Nations qui peuvent acquérir une demeure pour y loger leur famille? Pas étonnant non plus que peu d'entreprises aient pu se développer et contribuer au développement des communautés des Premières Nations.

On s'étonne souvent de l'ampleur des budgets consentis aux conseils de bande des réserves, prenant à témoin la situation de municipalités de taille comparable. On oublie pourtant que les conseils de bande ont la responsabilité d'assurer beaucoup plus de services que ceux habituellement assumés par les municipalités non soumises à la Loi sur les Indiens, notamment dans les domaines des services de santé, d'éducation et des services sociaux, qui sont pris en charge par le gouvernement provincial. En matière d'habitation, les restrictions précédemment décrites obligent les conseils de bande à assumer le financement et la gestion du logement pour la majorité de leur population. Ainsi, plus de $80 \%$ des dépenses pour les programmes autochtones du ministère des Affaires autochtones et du Développement du Nord du Canada sont liées à des services de base, fournis aux autres Canadiens par les gouvernements provinciaux et territoriaux et par les administrations municipales.

Preuve que ces sommes sont nettement insuffisantes? L’envoyé de l'ONU dépêché à l'automne 2013 pour enquêter sur les conditions de vie des populations autochtones au pays résumait la situation en ces termes: «Le Canada se classe constamment parmi les meilleurs pays pour son indice de développement humain, et pourtant, [...] les peuples autochtones vivent dans des conditions comparables à celles des pays qui arrivent plus bas dans ce classement.»

Déjà en 2011, la vérificatrice générale du Canada (chargée de l'audit des ministères et programmes fédéraux) faisait le constat que dans les réserves, «les éléments essentiels de l'existence, soit l'éducation, l'accès à l'eau potable et à un logement décent, sont radicalement inférieurs aux normes, et ce, de façon persistante. Les conditions de vie dans de nombreuses réserves sont mauvaises et ne se sont pas améliorées de manière substantielle depuis la dernière décennie». Le rapport de la vérificatrice générale nous rappelle également que cette situation ne résulte pas d'une mauvaise volonté de la part des communautés, mais bien de la structure même des programmes offerts: «Bon nombre des problèmes auxquels les Premières Nations se heurtent sont causés [...] par des obstacles structurels 
qui nuisent gravement à la mise en œuvre (des programmes)». Parmi les obstacles recensés, l'on notait l'absence de cadre législatif pour assurer la qualité des services fournis par le fédéral dans des domaines habituellement de juridiction provinciale ou municipale (éducation, santé ou eau potable).

En outre, les mécanismes mêmes de financement ne seraient pas appropriés, selon $\mathrm{M}^{\mathrm{me}}$ Fraser, car non seulement le calendrier de versement des contributions octroyées pose problème (la plupart des accords devant être reconduits annuellement), mais aussi, le fardeau administratif qui se rattache à ce mode de fonctionnement est extrêmement lourd, surtout pour les petites collectivités de Premières Nations qui disposent de moyens administratifs limités. Cette situation crée une incertitude pour les Premières Nations et complique la planification à long terme. En somme, plusieurs des réserves souffrent d'un manque d'expertise et ne peuvent pas répondre aux exigences administratives liées à la mise en œuvre de programmes clés.

En l'absence des services de base auxquels tous les autres citoyens canadiens ont droit, ainsi que des conditions structurelles nécessaires permettant aux communautés de se développer, comment s'étonner que ces populations ne puissent assurer à leurs membres les conditions de vie qui font des sociétés en santé?

\section{Les conditions d'une prise en charge réelle}

Les politiques colonisatrices canadiennes ont eu des répercussions considérables sur tous les aspects de la vie des populations autochtones. Ces structures sont encore présentes aujourd'hui. L'exemple des nombreuses contraintes imposées par la Loi sur les Indiens aux populations des Premières Nations est éloquent, puisqu'une telle loi entrave non seulement le droit d'accès au territoire et donc la pratique d'activités traditionnelles, mais aussi la libre circulation et l'exploitation des ressources naturelles.

Or, pour qu'une société puisse «se prendre en main», encore faut-il qu'elle puisse disposer des moyens pour ce faire. Pour inverser l'effet de plus d'un siècle de politiques assimilatrices sur la santé de leurs populations, les communautés autochtones doivent pouvoir reprendre le contrôle des facteurs qui l'influencent, bien au-delà de l'accès aux services. Tant qu'une société est laissée en marge de tout développement économique durable, tant qu'elle n'a pas le contrôle de ses propres ressources naturelles, 
ni le contrôle de son système d'éducation et de l'enseignement de sa langue, elle ne peut pas vivre correctement. Au mieux, elle ne peut que survivre.

L’on pourrait s'attendre à ce que les Québécois, plus que tout autre peuple au Canada, puisse démontrer une compréhension intuitive de ces phénomènes sociologiques universels. La réelle "prise en charge» des populations autochtones passera en grande partie par l'autodétermination et la reconnaissance de leurs droits au territoire. De fait, tel que nous le rappelle l'instance permanente sur les questions autochtones des Nations Unies, l'autodétermination et le développement humain et économique durable ne sont en fait que les deux côtés d'une seule et même médaille.

\section{Pour aller plus loin}

Affaires autochtones et Développement du Nord Canada. (1996). Points saillants du rapport de la Commission royale sur les peuples autochtones. http:// www.aadnc-aandc.gc.ca/fra/1100100014597/1100100014637: Gouvernement du Canada.

LePage, P. (2009). Mythes et réalités sur les peuples autochtones, $2^{e} e ́ d i t i o n$. Commission des droits de la personne et des droits de la jeunesse, Québec.

Nations Unies (2008). Déclaration des Nations Unies sur les droits des peuples autochtones. http://www.un.org/esa/socdev/unpfii/documents/DRIPS_fr.pdf. 



\section{LA RECHERCHE EN SANTÉ}





\title{
La recherche médicale en Afrique est un moyen pour l'Occident de tester des médicaments dangereux
}

\author{
Ashley Ouvrier
}

La recherche médicale - dont l'essai clinique est la procédure la plus emblématique - est un bras essentiel de la médecine sans lequel elle ne pourrait aujourd'hui prétendre avancer. Lorsqu'elle est conduite en Afrique, elle donne pourtant fréquemment lieu à des controverses ${ }^{1}$ qui réduisent ses enjeux à un combat entre David et Goliath. Celle-ci est en effet fréquemment associée à un scénario tout tracé, qui situe son principal problème dans le désir des industriels occidentaux de profiter de la vulnérabilité des populations pauvres du continent pour expérimenter leur produit afin de le revendre à prix fort sur le marché du Nord. C’est sensiblement dans cette perspective que l'écrivain John le Carré a élaboré son intrigue kenyane, La Constance du jardinier, qui fut un succès littéraire avant d'être adaptée au cinéma ${ }^{2}$. Or, envisager la recherche en Afrique comme un phénomène qui n'est qu'entre les mains des desiderata

1. Comme en témoigne le procès qu'a intenté l'État de Kano, au Nigeria, contre l'industriel du médicament américain Pfizer ou encore la suspension des essais cliniques en pré-exposition du VIH au Cameroun et au Cambodge, qui furent des événements largement documentés dans la presse.

2. John le Carré et le réalisateur Fernando Meirelles retracent dans leurs œuvres respectives l'enquête que mène un diplomate anglais à la suite de l'assassinat de sa femme alors que celle-ci s'apprêtait à dénoncer les malversations d'un essai clinique réalisé au Kenya. 
des industriels du Nord ne rend compte ni de la variété des acteurs de ce champ ni de la complexité des recherches qui s'y déroulent. Plusieurs a priori nous semblent sous-jacents à cette idée reçue selon laquelle la recherche médicale en Afrique ne serait qu'un moyen pour l'Occident de tester abusivement des molécules dangereuses sur des populations vulnérables. Nuançons cette vision à partir d'exemples concrets.

En Afrique comme dans les pays du Nord, la recherche médicale est d'une grande variété, tant par ses formes que par ses objectifs. Les essais, qui sont divisés en quatre étapes progressives ${ }^{3}$, ne présentent pas les mêmes risques pour les participants. La plupart des essais de phase 1 (ceux qui présentent le plus de risques) sont d'ailleurs réalisés dans les pays du Nord, et ce sont donc plutôt les phases 2 et 3 qui sont réalisées en Afrique. Depuis une trentaine d'années, les industriels du Nord réalisent de plus en plus d'études dans les pays en voie de développement et les pays émergents, mais cette dynamique s'accompagne également d'une augmentation du nombre de recherches réalisées dans le cadre de partenariats privés-publics. Ces nouveaux consortiums, à l'image du Meningitis Vaccine Project, qui associe l'Organisation mondiale de la santé, la fondation Bill et Melinda Gates et le laboratoire GlaxoSmith \& Kline, réussissent parfois à mettre à disposition des nouvelles thérapeutiques accessibles aux gouvernements des pays pauvres. Ainsi, grâce à ce collectif, un nouveau vaccin produit en Inde et vendu à moins de 300 francs CFA par dose (soit 50 centimes d'euros) permet aujourd'hui aux pays aux ressources limitées en Afrique de se prémunir contre la méningite, qui tue régulièrement sous forme d'épidémies parfois dévastatrices. Parmi les nombreuses études réalisées sur le continent, certaines sont appelées «essais de stratégie thérapeutique ${ }^{4} »$. Elles présentent peu de risques pour les participants, puisqu'elles se font à partir de médicaments qui ont déjà obtenu l'autorisation de mise sur le marché, mais elles permettent de

3. La phase pré-clinique consiste à tester la molécule préalablement identifiée en laboratoire sur des animaux. La première phase mesure l'innocuité du produit chez l'homme en bonne santé (dose maximale tolérée). La deuxième phase évalue la tolérance chez l'homme malade (posologie utile). Et enfin, la troisième phase mesure l'efficacité du produit chez l'homme malade (mode d'emploi, utilisation chez la femme enceinte, l'enfant, contre-indications) (Chippaux, 2004).

4. Les essais de stratégie thérapeutique consistent à tester une molécule ou une combinaison de molécules qui ont déjà obtenu une autorisation de mise sur le marché dans un pays autre que celui concerné par l'étude ou pour une autre pathologie. 
rendre accessibles des traitements ou des combinaisons de médicaments moins chers et plus efficaces en prouvant leur efficacité auprès d'une population. Par exemple, le Sénégal a étudié l'efficacité des médicaments antirétroviraux ainsi que leur observance alors que la communauté internationale résistait à rendre accessibles ces médicaments sur le continent, prétextant, entre autres, que les patients subsahariens étaient peu observants.

Toute la recherche sur le continent ne peut évidemment pas se résumer à ce type de succès, car certaines recherches très controversées sont aussi menées. Mais les investissements étrangers en matière de recherche médicale ne peuvent être réduits à un bloc monolithique de mauvaises intentions dont les populations du Sud ne pourraient être que les victimes. Le marché des pays pauvres n'est pas un non-marché, il est cependant un marché du médicament différent de celui du Nord. Les organismes supranationaux comme l'OMS, l'UNICEF ainsi que de nombreux services de coopération y sont d'importants financeurs et acteurs. Les populations du Sud sont dans ce contexte des partenaires ministériels et des équipes de recherche scientifiques qui sont en mesure de négocier et d'infléchir des décisions liées aux recherches menées sur le continent. Prendre le parti que la recherche médicale en Afrique n'est qu'à la merci des industriels, c'est aussi ignorer ou sous-estimer le travail des organismes de régulation du marché global du médicament comme Médecins sans frontières, qui soutiennent vigoureusement, avec d'autres groupes du Sud et du Nord, le droit à un accès aux médicaments pour les plus pauvres.

Derrière cet a priori d'une recherche forcément mal intentionnée sur le continent qui semble vouloir défendre le point de vue des plus faibles, on peut se demander s'il ne se cache pas aussi un vieux cliché, celui qui conduit à systématiquement considérer la figure de l'Africain comme celle de la victime ou de l'opprimé. Ainsi, on oublie souvent que les médecins et politiciens qui encadrent, soutiennent et autorisent les études réalisées sur le continent sont aussi des citoyens africains. Des études anthropologiques de plus en plus importantes montrent la manière dont les chercheurs et le personnel paramédical africain se saisissent avec finesse des moyens et des ressources des essais cliniques pour répondre à leurs besoins en matière de santé et de développement. La recherche médicale constitue parfois un moyen pour ces derniers de pallier les failles de leur système de santé, de rendre accessibles des traitements ou des soins de 
qualité à leurs patients démunis, ou encore de mieux rémunérer des cliniciens ou chercheurs locaux dans une politique de résistance à la fuite des cerveaux africains vers l'Europe ou les États-Unis. Ces appropriations de la recherche médicale sur le continent rend, cependant, les frontières entre recherche, développement et soins parfois très poreuses. Cette situation pose évidemment des questions sur le sens et les limites de la recherche médicale en Afrique.

La recherche médicale sur le continent présente ainsi un ensemble d'inconvénients et d'avantages compte tenu des contextes dans lesquels elle est réalisée. Ceux-ci doivent donc être rigoureusement analysés avant de crier au complot ou au scandale. Si le scénario de John le Carré et les analyses de type complotiste sont populaires, la réalité montre que les pratiques de la recherche sur l'homme en Afrique et ses enjeux sont plus complexes. Si, bien sûr, il est essentiel d'être vigilant et de condamner les recherches médicales qui ne respectent pas les recommandations internationales en matière d'éthique, il nous semble tout aussi important de faire remarquer les études cliniques potentiellement bénéfiques pour les patients du continent. Comme en témoignent les nombreuses études réalisées dans les pays du Sud, la recherche est un outil d'innovation thérapeutique et d'espoir dans le traitement de nombreuses endémies (paludisme, hépatites, méningite, tuberculose, VIH/sida, etc.). Il semble important de ne pas condamner cette activité, problématique au Nord comme au Sud depuis sa création, du fait de la pauvreté ou de l'origine de ses participants.

\section{Pour aller plus loin}

Chippaux, J.-P. (2004). Pratique des essais cliniques en Afrique. Paris: IRD Editions.

FAIRHEAd, J., M. Leach et al. (2006). Public engagement with science? : Local understanding of vaccine trial in Gambia. Journal of Biosocial Science, 38(1): 103-116.

Ouvrier, A. (2014). Faire de la recherche médicale en Afrique - ethnographie d'un village-laboratoire sénégalais. Karthala, IRD Editions. 


\title{
Des comités garantissent le caractère éthique des recherches en santé
}

\author{
Béatrice Godard et Matthew Hunt
}

Les comités d'éthique de la recherche ont été créés pour promouvoir les normes d'éthique ${ }^{1}$ les plus rigoureuses dans la conduite de recherches faisant appel à des êtres humains. Ces normes ont été édictées à la suite d'expériences médicales conduites sur des patients sans leur consentement ou sans se préoccuper de leur santé, ou encore sans se soucier si certaines études répondaient aux besoins et aux intérêts des communautés. Ces normes reposent sur les principes moraux ou éthiques que sont le respect de la personne, la bienfaisance, la justice sociale. En pratique, elles se traduisent par l'obtention du consentement, écrit ou oral, des participants à une étude et par l'évaluation par les chercheurs des bénéfices et des risques de l'étude. Elles concernent de fait la participation de personnes ou de leurs communautés et s'associent à un recrutement des participants juste et équitable pour la conduite d'une étude. Une des attentes consiste en ce que les bénéfices et les risques de l'étude soient distribués équitablement et que les buts et les valeurs de l'étude soient alignés sur

1. L'éthique est l'art de diriger la conduite: comment répondre face à une situation pour laquelle il n'y a pas de solution optimale? Viser un comportement éthique, c'est déterminer quelle est la meilleure façon d'agir dans un contexte donné. Il peut y avoir, dans une situation concrète, plusieurs possibilités de comportements éthiquement acceptables. 
ceux de la communauté afin de prévenir sa stigmatisation ou sa marginalisation. Ces normes sont reconnues à l'échelle internationale. Elles sont même parfois intégrées dans des lois ou règlements.

Ces normes permettent de guider les chercheurs, qu'il s'agisse d'études biomédicales, comportementales, épidémiologiques ou en sciences sociales. En effet, elles les guident pour protéger les personnes qui participent à leurs études, mais aussi pour que leur recherche soit utile à la société. Dès lors que des personnes participent à une étude, il est attendu qu'elle aboutisse à des connaissances scientifiques importantes qui soient communiquées à l'ensemble de la société et qui contribuent au bien-être de la société en général.

C'est justement cet attendu qui fait que, malgré l'approbation par un comité d'éthique de la recherche, celui-ci ne peut pas garantir le caractère éthique de l'étude. Nous passerons en revue quatre idées largement répandues sur les comités d'éthique de la recherche qui montrent que la protection des individus et des communautés n'est pas automatique, mais qu'il s’agit plutôt d'un idéal éthique à atteindre.

Les membres du comité d'éthique de la recherche ont l'expertise et l'autonomie suffisantes pour évaluer le caractère scientifique et éthique des projets de recherche qui leur sont soumis.

- Tout comité d'éthique de la recherche doit être composé de membres réunissant différentes expertises. Or, la recherche scientifique est complexe, pouvant faire appel dans une même étude à différentes disciplines et méthodologies. Cela demande que les membres comprennent bien la science derrière le projet, ce qui n'est pas toujours possible, dès lors que ceux-ci ne peuvent pas être experts dans tous les domaines.

- Pour prévenir les risques de certaines études, il est souvent recommandé d'effectuer un suivi des études. Ceci nécessite des ressources financières et humaines que la plupart des comités d'éthique n'ont pas. Le nombre élevé des demandes d'approbation de projets de recherche est une autre limite au suivi des études en cours.

- Quant à l'indépendance des comités d'éthique de la recherche, elle est relative: le financement de certaines études va parfois permettre aux participants d'accéder à des services et le refus d'approuver une 
étude, soit pour des motifs scientifiques, méthodologiques ou éthiques risque donc de pénaliser ces participants. De même, le refus d'approuver une étude dirigée par un collègue risque d'entraîner des conflits potentiels. Ou encore, une étude refusée par un comité d'éthique peut être approuvée par un autre comité. De nombreux comités d'éthique se plaignent d'un manque de soutien institutionnel dans leur processus de décision.

Le comité d'éthique de la recherche garantit que l'étude va produire des résultats importants et significatifs.

- Les chercheurs sélectionnent les individus qui devraient faire partie de l'étude et ceux qui devraient en être exclus. Dans la recherche clinique, les critères d'exclusion sont de plus en plus nombreux afin de maximiser les chances d'obtenir des résultats. Ainsi, sont souvent exclues les femmes, les personnes âgées de moins de 40 ans ou âgées de plus 65 ans, les personnes qui fument, qui prennent des médicaments, etc. Ces critères d'exclusion sont appliqués presque systématiquement, même si l'argument pour l'exclusion est rarement remis en question. Ce phénomène exclut un nombre important d'individus qui pourraient bénéficier des résultats de ces recherches, et la généralisation de ces résultats à l'ensemble de la communauté peut être remise en cause. De telles études risquent de produire des résultats partiels ou non fiables.

Le comité d'éthique de la recherche garantit que les chercheurs vont rapporter les résultats aux participants et à la communauté.

Il est de plus en plus demandé aux chercheurs de transmettre les connaissances scientifiques en divulguant leurs résultats, en rendant leurs publications accessibles. À nouveau, voici quelques exemples de biais de publication pouvant avoir des conséquences éthiques:

- Report des résultats négatifs: les auteurs et les éditeurs des revues scientifiques ont plus tendance à publier des expériences ayant obtenu un résultat positif que celles ayant obtenu un résultat négatif ou nul. Cela donne aux lecteurs une perception biaisée de l'état de la recherche. En effet, les chercheurs tendent à considérer un résultat 
négatif ou non significatif statistiquement comme moins important, car ils craignent que leur article soit refusé par la revue ou que leurs résultats limitent la mise en marché d'une innovation scientifique. Ils préfèrent dans de tels cas ne pas publier ces résultats. D’autres chercheurs sont tenus au secret industriel et ne peuvent donc pas publier certains résultats de recherche. Les biais de publication constituent un manque de transparence et peuvent influencer la traduction de la recherche en pratique, au risque d'engendrer des préjudices pour les individus et le public. Par exemple, en sciences biomédicales, cela peut influencer les décisions de prescriptions des médecins ou entraîner une méprise thérapeutique de la part des patients.

- Promesses exagérées: plusieurs auteurs d'articles scientifiques clament les bénéfices potentiels des résultats de leurs études, ce qui entraîne parfois des attentes démesurées de la part de personnes à risque ou souffrant de maladies. Des précautions devraient être prises pour éviter les risques associés au battage publicitaire et à la diffusion prématurée des avantages potentiels d'une recherche.

D’autres facteurs liés à la diffusion des résultats de recherche peuvent avoir des implications éthiques. C'est le cas lorsque les chercheurs n'ont pas les ressources pour faire traduire leurs articles en anglais, pour payer les frais de publication ou pour que leurs publications soient répertoriées dans des bases de données internationales. La conséquence est que ni la communauté scientifique ni le public ne peuvent bénéficier des connaissances de ces recherches, de même que les besoins des personnes ou des groupes sur lesquels ont porté ces recherches peuvent être plus difficilement connus et étudiés et des interventions mises en place.

L'approbation d'une étude par un comité d'éthique de la recherche garantit qu'elle contribue au bien-être d'une communauté ou de la société en général.

Même si l'utilité sociale est devenue un critère important dans l'approbation scientifique et éthique d'une étude, de nombreuses études sont financées et n'apportent pas vraiment de nouvelles connaissances:

- C’est le cas des études visant à produire des médicaments génériques. L'argument que la production de ces médicaments contribue à en 
diminuer le coût est contesté. De plus, du fait que des volontaires sains participent à ces études principalement parce qu'ils sont payés pour le faire, on constate que ces volontaires sont très souvent vulnérables économiquement et on craint que certains pourraient se porter volontaires trop souvent et devenir des bénévoles professionnels. Le risque d'une interaction médicamenteuse et d'une anémie de prélèvement de sang excessif demeure.

D'autres études sont financées et n'apportent pas de valeur sociale à la communauté qui fait l'objet de l'étude:

- C'est le cas d'études faites auprès d'individus et de groupes qui ne bénéficient pas des résultats subséquents de l'étude, par exemple après la mise au point de thérapies. En plus d'avoir fait subir les inconvénients, ces études risquent de renforcer des inégalités plutôt que de les diminuer.

- Il y a aussi les effets contraires de la recherche sociale, notamment sur l'exclusion, les discriminations et la pauvreté. Il se peut que les résultats de la recherche, une fois médiatisés, pénalisent davantage la population, alors que le but était totalement autre. Ces effets peuvent toucher les populations étudiées en augmentant, par exemple, leur déqualification aux yeux de l'opinion publique; à son tour, l'opinion publique pourra devenir plus hostile et craintive devant les populations démunies; pour les législateurs et les agents de répression, la recherche sociale pourra donner plus de légitimité au contrôle social et à la coercition.

Il est toutefois possible de réduire les risques liés aux limites méthodologiques ou à la diffusion des résultats de recherche ou encore à leurs retombées sociales. Les comités d'éthique à la recherche y contribuent en appliquant des normes, lesquelles reposent sur des principes ou valeurs aujourd'hui bien établis. La formation en éthique des chercheurs, ainsi que la prise de conscience des citoyens, y contribuent également. Les limites des valeurs éthiques occidentales sont aussi de plus en plus reconnues et de nouvelles façons de faire de la recherche en partenariat avec les communautés sont privilégiées, afin de partir de leurs besoins, de tenir compte de leurs expériences et de leurs perspectives sur les causes 
et les solutions aux problèmes de recherche biomédicale, comportementale, épidémiologique ou en sciences sociales.

Certes, de nombreuses questions restent non résolues pour garantir le caractère éthique des recherches et de nouveaux problèmes surgissent au fur et à mesure de l'avancement des connaissances. C'est presque le sort de la recherche scientifique en constante marche vers l'inconnu, d'où l'importance d'avoir des balises et des normes éthiques rigoureuses. Mais aucun comité d'éthique de la recherche ne saurait constituer un moyen suffisant pour assurer une conduite éthique.

Rabelais disait déjà à son époque: «Science sans conscience n’est que ruine de l'âme.» Dans ce message, il nous enseigne que la recherche scientifique et l'éthique de la recherche n'ont pas de raison d'être séparées. Elles devraient pouvoir s'informer et se nourrir mutuellement.

\section{Pour aller plus loin}

Bastien, R., Tremblay, L. et Dumais, L. (2010) Stigmatisation "paradoxale» induite par la recherche sociale sur l'exclusion: discussion à partir du cas des conduites violentes chez des enfants sur le chemin de l'école. Cahiers du LAREPPS, ${ }^{\circ}$ 10-07.

Godard, B. et Moubé, Z. (2013) Construire et enseigner la bioéthique dans les pays francophones: au carrefour des disciplines et des pratiques. J Int Bioéthique, 24: $53-72$.

Mondain, N., et Arzouma, É. B. (dir.) (2012) La recherche en contexte de vulnérabilité -engagement du chercheur et enjeux éthiques. Paris: L'Harmattan, coll. Logiques sociales. 


\title{
Les objectifs, les séminaires et les recommandations permettent d'améliorer la santé des populations
}

\author{
Yannick Jaffré
}

La santé mondiale correspond à l'assemblage complexe de choix stratégiques, de priorités provenant, de manière complexe, d'une gouvernance mondiale se manifestant par des mises à l'agenda de questions prioritaires, et des mises en ouvre de programmes techniques conduits par de multiples organisations internationales, ONG, associations locales, officines de conseil...

Ce dispositif est largement pensé et présenté comme un kit de pièces harmonieusement disposées sous la forme d'une pyramide. Des recommandations, officiellement basées sur des données probantes, sont censées inciter à la définition de politiques efficaces et efficientes, celles-ci devant à leur tour entraîner des actions concrètes de terrain.

Du haut décisionnel au bas bénéficiaire, le progrès médical est alors censé se diffuser harmonieusement grâce à des orientations et des incitations déclinées en objectifs, puis à des formations ou des programmes en cascade apportant les lumières de la santé publique, là où règnerait encore l'obscurité des choix politiques locaux et la confusion des pratiques populaires.

Certes, nous caricaturons un peu. Définir des priorités est nécessaire. De même, transmettre des connaissances est indispensable, et les acteurs 
des institutions de développement ne sont pas si candides. Nombreux sont ceux qui ne partagent pas cette vision naïvement scientiste et largement post-coloniale du progrès. Autrement dit, les modalités des programmes sont plus complexes que nous ne pouvons l'illustrer dans ce très bref chapitre. Cependant, quelques traits récurrents constituent - sur le mode du Bourgeois gentilhomme qui, voulant exprimer son amour, ne pouvait que distribuer diversement les termes finis de son compliment ${ }^{1}$ - l'ossature intellectuelle et l'organisation pratique des programmes de développement.

Tout d'abord, la problématique générale des actions de développement est largement orientée par des termes comme ceux de barrières culturelles ou d'obstacles. Ces métaphores bornent la réflexion en induisant l'idée qu'il suffirait de sensibiliser, de pousser une sorte de porte langagière ou symbolique pour accéder à une autre culture, où enfin les populations agiraient selon les diverses rationalités comportementales définies et proposées par les programmes sanitaires, économiques, agronomiques ou éducatifs.

Les bailleurs de fonds confortent largement cette vision mécanique des transformations sociales par diverses procédures. La principale consiste à obliger les initiateurs de projets de santé mondiale à programmer leurs actions selon des procédures de codification comme les résolutions par objectifs ou autres cadres logiques. Ils doivent construire, de rubriques à remplir en cases de programmation, une narration implicite mettant en lien «une série de relations logiques entre des objectifs hiérarchisés, les moyens mis en œuvre pour les atteindre, les conditions externes indispensables, et les résultats attendus».

L'étape suivante, marquant le véritable début des projets sur le terrain, est souvent initiée par des séminaires assortis de recommandations censées fixer à la fois les objectifs de l'action et exprimer la ferme et unanime résolution des acteurs à les accomplir.

Très souvent, vient ensuite une phase d'expérimentation. Quelques programmes pilotes concentrant des moyens, des compétences et souvent objets d'enjeux scientifiques et de carrières sont censés démontrer, dans le réel, la justesse des propositions effectuées.

1. Ce que décline le Maître de philosophie: «Belle Marquise, vos beaux yeux me font mourir d'amour. Ou bien: D'amour mourir me font, belle Marquise, vos beaux yeux. Ou bien: Vos yeux beaux d'amour me font, belle Marquise, mourir. Ou bien: Mourir vos beaux yeux, belle Marquise, d'amour me font. Ou bien: Me font vos yeux beaux mourir, belle Marquise, d'amour» (Molière, Le Bourgeois gentilhomme: Acte 4 - Scène II). 
Enfin, cette démonstration est faite sur un petit territoire - idéalement, il ne reste qu'à "passer à l'échelle » pour qu'un pays, voire un continent, bénéficie ainsi des lumières de la science éclairant l'inébranlable volonté des hommes!

Ainsi, réduit à sa maigre ossature, ce prêt-à-penser se révèle bien naïf. Pourtant, il circonscrit très concrètement ce qui est recevable par la plupart des institutions, des bailleurs de fonds, voire des institutions savantes. Il définit aussi les discours légitimes pour conquérir des positions institutionnelles et obtenir du financement. Il nourrit enfin toute une technologie, cabinets d'audits, experts, consultants, partenaires de projets, mêlant dans la plus grande confusion des modèles psychosociaux et diverses théories de la communication et de l'évaluation.

\section{Des hauts et des bas}

Le paradoxe est que personne ne croit véritablement à ces hypothèses et procédures. Certes, chacun s'accorde sur la nécessité de programmer des actions et des moyens idoines pour les réaliser, de disposer d'une feuille de route, et pourquoi pas, de se donner un but en évoquant des lendemains qui chantent, que ce soit au passé de la «santé pour tous en l'an 2000 », ou au futur des «objectifs mondiaux du développement». Mais soyons réaliste.

D’un point de vue institutionnel, il est aisé de constater que ce dispositif perdure pour diverses raisons, allant des plus larges aux plus pragmatiques. Il s'agit de construire une fiction crédible du développement, d'éviter d'évoquer les raisons politiques (nationales et internationales) des situations sociosanitaires observées, de justifier des choix économiques externes aux sociétés bénéficiaires, de décaisser rapidement des budgets, de toucher des per-diem, de prendre de «bonnes résolutions humanitaires», de justifier sa vie ou sa retraite en «aidant les autres», de ne pas scier la branche idéologique justifiant un confortable salaire... Bref, un plein d'avantages politiques, pécuniaires, moraux et affectifs.

D’un point de vue scientifique, les critiques concernant les implicites de ce dispositif développementiste ne sont pas moins nombreuses. La culture, loin de correspondre à un ensemble de rôles figés, spécifiques d'une supposée communauté homogène et consensuelle, correspond plutôt aux multiples façons dont divers segments - le plus souvent antagoniques - de populations négocient des pouvoirs et résolvent concrètement des 
problèmes dans des environnements spécifiques. Par exemple, qui va bénéficier principalement d'une adduction d'eau? Qui va accéder à des formations pour devenir agent de santé communautaire bénévole et ainsi pouvoir espérer obtenir un emploi pérenne et rémunéré? De même, si des représentations sociales peuvent inciter à agir d'une certaine façon, ces systèmes de sens et ces rumeurs informelles n'ont jamais empêché les populations d'agir pragmatiquement et souvent selon des conduites rationnelles en finalité. Par exemple, ce n'est pas une certaine représentation du corps et de la grossesse qui éloigne les parturientes des maternités, mais la qualité, l'accès et le coût des soins.

Par ailleurs, les identités des acteurs sociaux débordent toujours les fonctions qu'ils occupent et les rôles qu'ils jouent dans leurs vies professionnelles. Il en résulte que les conduites des populations ou des professionnels procèdent bien plus du jeu de contraintes adverses ou de l'articulation contradictoire de variables discontinues (par exemple le pouvoir des belles-mères + le manque de moyens de communication + l'absence d'un soignant peuvent entraîner un décès) que d'un simple manque de savoir ou de lucidité sur les tâches à accomplir. Très simplement, même si l'on s'est engagé, lors d'un séminaire ou de son serment d'Hippocrate, à soigner les plus pauvres, en l'absence de salaires réguliers, si l'on souhaite disposer d'argent pour nourrir et soigner les siens, il faut bien être indélicat avec les ressources d'un service ou d'une association...

De plus, si les interventions de santé mondiale passent, sont largement amnésiques et bien peu historiquement responsables des actions engagées, les populations restent. Elles se souviennent, évaluent dans la durée les actions dont elles ont bénéficié. Mais comment celui qui, il y a vingt ans, avait vanté les mérites des soins de santé primaires, puis il y a dix ans, l'indispensable politique de recouvrement des coûts, peut maintenant se déclarer en faveur de l'exemption de paiement? Comment expliquer que la distribution de nourriture aux mères d'enfants malnutris était une bonne chose, puis qu'il ne fallait surtout plus le faire, puis que l'on va maintenant lutter contre la pauvreté en distribuant des ressources (transferts monétaires) aux familles ayant de jeunes enfants? Fort pragmatiquement, pour les populations, l'occasion fait le larron, et chacun tente de maximaliser ses ressources en usant des interventions dont bénéficie maintenant presque chaque village. Mais quelle est la crédibilité de ces interventions? Ce sont des affaires de Blancs, dit-on à tort et à raison. 
Enfin, lors de programmes pilotes, les résultats obtenus en un seul lieu n'ont souvent qu'une valeur propre à ce lieu et ne sont pas généralisables aux problèmes d'un pays en développement, notamment lorsque le financement, les compétences mobilisées et les enjeux ne sont plus similaires.

\section{Penser et agir autrement}

Affublés d'une étrange cécité et d'une amnésie sélective, les développeurs finissent par prendre leur programme pour le tout du réel, excluant le reste - langues, pouvoirs, politiques, religions, histoire - comme n'étant que le contexte de l'intervention.

Et pourtant, ce contexte, ce que l'on ne s'est pas donné la peine d'analyser, oublié dans les marges des projets, est ce qui constitue les raisons d'agir des acteurs. Faut-il alors s'étonner que ces dimensions sociales, chassées des séminaires et des programmes de papier, reviennent dans le déroulement concret des actions?

Partir du réel des situations, analyser les contraintes des acteurs, diffuser de véritables connaissances sans souci d'immédiates évaluations, conduire des actions non contradictoires sur de longues durées, contribuer à une certaine réflexivité des acteurs... Tout ceci constituerait un autre paradigme, non pour développer, mais pour accompagner lucidement des dynamiques économiques, politiques et sociales maintenant mondialisées.

\section{Pour aller plus loin}

Balandier, G. (1971). Sens et puissance, les dynamiques sociales. Paris, PUF.

Giovalucchi, F. et Olivier de Sardan, J.-P. (2009). Planification, gestion et politique dans l'aide au développement: le cadre logique, outil et miroir des développeurs. Revue Tiers Monde, $\mathrm{n}^{\circ}$ 198, avril-juin: 383-406.

JAfFré, Y. (2007). Quand la santé fait l'article. Presse, connivences élitaires et globalisation sanitaire à Bamako, Mali. Revue de pathologie exotique 100 (3) : 207-215. 


\title{
La présentation de preuves scientifiques aide les décideurs à agir rationnellement
}

\author{
Mathieu Ouimet et Pierre-Olivier Bédard
}

Les décisions en matière de politiques publiques résultent de la conjonction de ce que certains politologues désignent comme les trois «I», c'est-à-dire les institutions (les valeurs, les coutumes, les normes, les lois, les règles, etc.), les intérêts (la politique, les groupes de pression, etc.) et les idées émanant d'une multiplicité de sources (élus, groupes de pression, experts, etc.). Plusieurs experts soutiennent que les savoirs scientifiques mériteraient d'être davantage mobilisés dans les cercles où se dessine l'action publique. Cette mouvance est particulièrement populaire dans les pays où la mission de la fonction publique dépasse les activités administratives traditionnelles visant le respect des lois et la surveillance des comptes publics. C'est le cas dans les pays où les activités d'analyse et d'évaluation de l'action publique sont pratiquées régulièrement (comme la GrandeBretagne, les États-Unis, le Canada, l'Australie, etc.). Dans ce type de fonction publique, les analystes de politique jouent un rôle de courroie de transmission entre les lieux de production et de diffusion des savoirs scientifiques, d'une part, et les lieux où s'élaborent et se décident les actions publiques en réponse à des problèmes considérés comme prioritaires par les autorités compétentes, d'autre part.

L’idée selon laquelle les savoirs issus de la recherche scientifique sont une source à considérer, voire à privilégier, dans les cercles d'élaboration des politiques publiques se justifie par le fait que certaines questions ne 
peuvent trouver de réponse qu'en recourant à l'observation ou à l'expérimentation scientifique. Par exemple: le programme de dépistage a-t-il contribué à réduire l'incidence de cancer dans la population? Quels sont les principaux facteurs de risque de cette condition de santé dans la population? Quelle est la prévalence de cette condition dans la population? Que pense la population du programme de prévention? De façon générale, bien qu'elles ne soient jamais absolues et définitives, les réponses qui découlent de recherches scientifiques ont le mérite d'être moins arbitraires que celles qui émanent de l'intuition ou de l'imagination de participants à des séances de remue-méninges.

Bien que la contribution potentielle des savoirs scientifiques à l'analyse des politiques soit généralement reconnue, la mobilisation de ces savoirs par les agents et responsables de l'État bute sur plusieurs obstacles. Premièrement, le calendrier des responsables de politiques est souvent plus court que celui des scientifiques. La méthode juste-à-temps ou zérodélai est difficilement praticable dans les milieux scientifiques. Deuxièmement, des différences culturelles entre l'appareil étatique et le milieu scientifique peuvent parfois nuire à l'établissement de liens de collaboration et de confiance. Ce qui est pertinent dans un milieu l'est peut-être moins dans l'autre. Enfin, une recension systématique des écrits publiée en 2014 a bien montré qu'une formation insuffisante en méthodologie de la recherche est l'un des principaux obstacles à la mobilisation des savoirs scientifiques au sein de l'administration publique.

Deux grandes perspectives sont généralement adoptées pour surmonter ces obstacles. La première consiste à promouvoir la production et la mise à disposition de recensions systématiques d'écrits scientifiques (version longue ou abrégée) adaptées aux besoins des usagers. Dans un monde idéal où de telles recensions existeraient pour toutes les questions d'intérêt, les analystes des ministères et organismes gouvernementaux n'auraient plus qu'à se procurer une copie d'une recension, à la lire et à en résumer les résultats dans une note de service destinée à informer leurs supérieurs hiérarchiques. Les acteurs qui s'inscrivent dans cette perspective réfléchissent également aux meilleures façons d'organiser la structure et la forme de ce type de document afin de répondre le mieux possible aux besoins des usagers.

La deuxième perspective pour surmonter les obstacles à la mobilisation des savoirs scientifiques au sein des organisations publiques se 
focalise davantage sur le processus de transfert des savoirs scientifiques plutôt que sur le produit à transférer. Par exemple, il sera question de réfléchir à des stratégies visant à stimuler la création de liens de collaboration entre les chercheurs et les utilisateurs. La pratique du courtage de savoirs scientifiques selon laquelle un courtier travaille à faciliter l'établissement de liens d'échange avec le milieu de la recherche fait partie des nombreuses stratégies proposées.

Quelle que soit la perspective adoptée, il est généralement admis, de façon implicite, que l'utilisateur est en mesure d'agir de façon rationnelle. Autrement dit, on suppose qu'il est à même d'atteindre ses objectifs lorsqu'il est exposé aux incitations adéquates et lorsqu'il bénéficie d'occasions d'apprentissage. En bref, il est généralement admis que la théorie du choix rationnel fournit une description appropriée du comportement du décideur. Le problème est que depuis les recherches empiriques en psychologie et en économie expérimentale, nous savons que cette théorie ne résiste pas aux tests empiriques.

Nous nous intéresserons ici aux deux éléments essentiels de la théorie du choix rationnel, c'est-à-dire les principes de dominance et d'invariance. Le principe de dominance est celui voulant que si l'option A est globalement supérieure à l'option $\mathrm{B}$, alors $\mathrm{A}$ devrait être préférée à $\mathrm{B}$. Prenons un exemple. Imaginons les deux options suivantes: A) transition vers une couverture universelle des soins de santé; B) maintien d'une couverture non universelle. Supposons que les responsables de politiques qui souhaitent améliorer l'état de santé générale de la population envisagent sérieusement de prendre une décision entre A et B. Supposons également qu'on les expose à des études scientifiques dont les résultats suggèrent fortement que l'option A est supérieure à l'option B. Suivant le postulat de dominance, les responsables politiques devraient donc préférer la première à la deuxième option. Pourtant, il existe une multitude d'exemples où les décideurs choisissent l'option dont l'efficacité est la moins bien rapportée sur le plan scientifique. Pourquoi? Les recherches empiriques montrent que le principe de dominance ne peut être respecté qu'en situation de transparence totale de l'information et des autres éléments nécessaires à la prise de décision (rappelons que les preuves scientifiques ne sont qu'une des multiples considérations intervenant dans le processus décisionnel). Or, un chercheur a bien montré que plusieurs informations pourtant nécessaires à la formation d'un jugement portant 
sur la valeur des études scientifiques ne sont malheureusement pas toujours présentées dans les publications. Le respect du principe de dominance est également problématique en ce qu'il est partiellement influencé par l'inadéquation d'un deuxième élément de la théorie normative du choix rationnel, c'est-à-dire celui de l'invariance.

L'élément de l'invariance veut que la préférence entre des options ne doive pas être influencée par leur description. En d'autres mots, les différentes façons de présenter une problématique de choix, dont les avantages et inconvénients de chacune des options, devraient conduire au même résultat, c'est-à-dire au même choix. Or, des expériences montrent que les décideurs auraient tendance à se laisser influencer par le cadrage de l'information. Une expérience désormais classique a bien montré que le fait de demander à des décideurs de choisir entre deux programmes de prévention dont les effets estimés sont présentés en termes de survie n'aura pas le même impact décisionnel que lorsque ces mêmes effets sont présentés en termes de mortalité. De plus, il est possible de cadrer l'information afin qu'elle donne l'illusion qu'une option, pourtant inférieure à une autre, apparaisse comme la meilleure option, rendant ainsi caduc le principe de dominance.

Un autre aspect également lié au phénomène de cadrage est malheureusement rarement évoqué par les experts en transfert de connaissances. Il s'agit de la théorie selon laquelle l'information à connotation négative contribue davantage à l'impression finale que se fera un individu d'une option ou d'un phénomène que l'information à connotation positive. Les implications pratiques de cette théorie dans le domaine du transfert des savoirs scientifiques sont majeures. Un des principes normatifs de la pratique fondée sur les données probantes est l'attitude critique envers les savoirs et les pratiques. Par exemple, selon la norme PRISMA (Preferred Reporting Items for Systematic Reviews and Meta-Analyses), toute recension systématique d'études scientifiques devrait présenter les biais méthodologiques de toutes les études scientifiques sélectionnées dans la recension. Imaginons que les résultats d'une recension suggèrent de façon quasiment univoque qu'une intervention est efficace et non nuisible. L'analyste de politique aurait donc de bonnes raisons de croire au caractère prometteur de l'intervention et d'en faire la promotion auprès de ses supérieurs. En revanche, imaginons que les auteurs de la recension, voulant se conformer au standard PRISMA, mettent en garde les lecteurs en 
dévoilant une imposante liste de biais identifiés dans les études recensées. En théorie, cette information négative devrait pondérer à la baisse l'impression d'efficacité de l'intervention. Cette tension entre la nécessité de transparence dans la présentation de résultats de recherche et la prise en compte des risques de biais par les utilisateurs potentiels devrait faire l'objet de plus amples investigations empiriques.

En somme, le transfert des savoirs scientifiques vers les cercles où se dessine l'action publique dans le domaine de la santé mondiale est un processus complexe et difficile à saisir. Les décideurs, comme tous les êtres humains d'ailleurs, ne sont pas seulement guidés par des motivations instrumentales et leurs décisions émanent rarement (voire jamais) d'un processus tel que celui qui est décrit par la théorie classique du choix rationnel. D'une certaine façon, ces éléments mériteraient d'être davantage pris en compte par les experts en transfert de connaissances.

\section{Pour aller plus loin}

IOANNIDIS, J. P. A. (2007). Limitations are not properly acknowledged in the scientific literature. Journal of Clinical Epidemiology 60(4):324-329.

Kahneman, D. (2012). Système 1/Système 2: Les deux vitesses de la pensée. Paris, Flammarion.

Martinez, F. (2010). L'individu face au risque: l'apport de Kahneman et Tversky. Idées économiques et sociales 161 (3): 15-23. 


\section{Les auteurs (par affiliation)}

Centre national de la recherche scientifique (CNRS) (France)

Josiane Tantchou

Centre national de la recherche scientifique, École des hautes études en sciences sociales (CNRS/EHESS), LASDEL (Niger)

Jean-Pierre Olivier de Sardan

Centre national de recherche scientifique, Institut des sciences des sociétés (CNRST/INSS) (Burkina Faso)

Sylvie Zongo

Clinique médicale l'Actuel (Canada)

David Lessard et Réjean Thomas

École des hautes études en santé publique (EHESP) (France)

Eric Breton

Groupe pour l'abolition des mutilations sexuelles féminines (Belgique)

Khadidiatou Diallo et Fabienne Richard

Institut national de la santé et de la recherche médicale (INSERM) (France)

Ashley Ouvrier

Institut national de la santé et de la recherche médicale (IRSEM),

Université Paris 13, (France)

Pierre Aïach 
Institut national de santé publique (INSPQ) (Canada)

Faisca Richer

Institut de médecine tropicale d'Anvers (Belgique)

Bart Criel et Fahdi Dkhimi

Institut de recherche pour le développement (IRD) (France)

Carine Baxerres, Alexandre Dumont, Jean-Yves Le Hesran,

Fatoumata Ouattara et Laurent Vidal

Institut de recherche pour le développement (IRD) (Burkina Faso)

Anne Attané et Florence Fournet

Organisation panaméricaine de santé (OPS/OMS) (Haïti)

Ludovic Queuille

Université d'Aix-Marseille (France)

Yannick Jaffré et Sandrine Musso

Université de Bordeaux (France)

Ramatou Ouédraogo

Université de Caen (France)

Emmanuel Bonnet

Université de Genève (Suisse)

Clémentine Rossier

Université de Heildeberg (Allemagne)

Stephan Brenner et Manuela De Allegri

Université de Montréal (Canada)

Loubna Belaid, Hélène Delisle, Sylvie Fortin, Béatrice Godard,

Solène Lardoux et Valéry Ridde

Université de Tulane (États-Unis)

Thomas Druetz

Université du Québec à Montréal (UQAM) (Canada)

Amara Bamba, Marie-Ėve Carle, Joseph Josy Lévy

et Elhadji Mamadou Mbaye 
Université du Québec en Outaouais (UQO) (Canada)

\section{Drissa Sia}

Université d'Ottawa (Canada)

Mamadou Barry, François Chiocchio, Marie-Hélène Chomienne, Katia Mohindra, Karine Savaria et Sanni Yaya

Université d'Oslo (Norvège)

Katerini T. Storeng

Université Laval (Canada)

Georges Batona, Pierre-Olivier Bédard, Aissatou Coulibaly, Sophie Dupéré, Laurence Guillaumie, Marie-France Labrecque, Jacky Ndjepel, Mathieu Ouimet et Nicolas Vornax

Université McGill (Canada)

Matthew Hunt et Bertrand Lebouché

Sida: Fonds pour la mémoire (France)

Michel Bourrelly 



\section{Table des matières}

$\begin{array}{ll}\text { Préface } & 7\end{array}$

$\begin{array}{ll}\text { Introduction } & 13\end{array}$

\section{LES POLITIQUES \\ ET LES SYSTÈMES DE SANTÉ}

$1 \quad$ L'implantation de la médecine occidentale devrait entraîner la disparition des médecines locales dans les pays à faibles revenus

2 Les politiques de santé ne se fondent que sur des critères rationnels

3 En Afrique, les soins gratuits déresponsabilisent la population, ne sont pas valorisés et sont de mauvaise qualité

4 Financer le système de santé sur la base des résultats sauve des vies

5 Les médicaments du marché informel sont des faux médicaments

6 Les missions internationales médicales bénévoles à court terme sont efficaces

7 Le VIH/sida pose des questions totalement inédites aux politiques de santé

8 Les conflits d'équipes sont essentiels à la performance des professionnels de la santé

9 Le syndrome d'épuisement professionnel du soignant n'existe pas en Afrique 


\section{LA PRÉVENTION ET LES SOINS}

10 Les génériques ne sont pas aussi efficaces que les médicaments de marque

11 Pour lutter contre la transmission du VIH, il suffit d'utiliser le condom

12 Les populations africaines ne suivent pas les consignes posologiques des traitements antirétroviraux

13 Les méthodes contraceptives rendent stérile

14 Le nombre d'avortements provoqués augmente quand on libéralise cette pratique

15 Les populations analphabètes ignorent l'importance des vaccins pour leurs enfants si bien qu'elles refusent de les faire vacciner 82

16 Le sucre est le seul responsable de l'épidémie mondiale de diabète de type 2

17 Les rapports sexuels avant une compétition nuisent à la performance sportive

\section{LA SANTÉ MATERNELLE ET INFANTILE}

18 Les femmes n'aiment pas annoncer leur grossesse par pudeur ou parce que cela porte malheur

19 C'est parce que les femmes sont ignorantes qu'elles accouchent encore à domicile

20 En Afrique, les femmes peuls n'accouchent pas à l'hôpital pour des raisons culturelles

21 La gratuité de la césarienne permet d'accélérer la réduction de la mortalité maternelle et néonatale en Afrique

22 Les mères africaines, par manque d'éducation, n'amènent pas leurs enfants au centre de santé

23 Les agents de santé communautaires peuvent soigner les enfants fébriles dans les régions rurales d'Afrique subsaharienne 


\section{LES POPULATIONS VULNÉRABLES ET L'ÉQUITÉ}

24 Les prestataires confessionnels servent les pauvres

25 La micro-assurance santé offre une protection sociale efficace pour les plus démunis

26 Les personnes vivant dans les villes sont privilégiées du point de vue de la santé

27 C'est sur les routes des pays les plus pauvres qu'on meurt le plus

28 Les femmes meurent plus tard que les hommes et sont plus malades

29 La réduction des inégalités sociales de santé passe principalement par des actions en faveur des plus vulnérables

31 C'est parce qu'ils sont migrants qu'ils ont la tuberculose

\section{LES SINGULARITÉs DES POPULATIONS}

32 L'obésité est un problème de riches dans les pays en développement

33 L'obésité concerne surtout les États-Unis d'Amérique 169

34 Pour se soigner, les sociétés africaines sont solidaires

35 Les Africaines font beaucoup d'enfants

36 L'homosexualité n'existe pas en Afrique

37 C'est à cause de leur culture que certains patients ne suivent pas leur traitement

38 Les hommes qui ont des relations sexuelles avec d'autres hommes sont largement responsables de la transmission du VIH

39 La participation des femmes au microcrédit explique la violence conjugale en Asie du Sud

40 Les femmes sont responsables de leur assassinat par leur partenaire 
41 L'excision est un rite initiatique pratiqué en Afrique, chez les musulmans et dans les villages

42 Avec tous leurs privilèges, les communautés autochtones devraient pouvoir prendre en charge leur santé

\section{LA RECHERCHE EN SANTÉ}

43 La recherche médicale en Afrique est un moyen pour l'Occident de tester des médicaments dangereux

44 Des comités garantissent le caractère éthique des recherches en santé

45 Les objectifs, les séminaires et les recommandations permettent d'améliorer la santé des populations

46 La présentation de preuves scientifiques aide les décideurs à agir rationnellement 
1939: l'alliance de la dernière chance. Une réinterprétation des origines de la Seconde Guerre mondiale $•$ Michael J. Carley

L'aide au conditionnel. La contrepartie dans les mesures envers les personnes sans emploi en Europe et en Amérique du Nord • Gérard Boismenu, Pascale Dufour et Alain Noël

L'aide canadienne au développement • François Audet, Marie-Eve Desrosiers et Stéphane Roussel

Amerindia. Essais d'ethnohistoire autochtone • Roland Viau

L'analyse des politiques publiques • Luc Bernier, Guy Lachapelle et Stéphane Paquin

Anatomie de la prison contemporaine. Nouvelle édition • Guy Lemire et Marion Vacheret

L'année politique au Québec. 1997-1998 • Robert Boily

Apprendre en situation d'autodidaxie $\bullet$ Nicole Anne Tremblay

Approches et pratiques en évaluation de programmes. Nouvelle édition revue et augmentée • Christian Dagenais, Valéry Ridde

L'armée canadienne vous parle. Communication et propagande gouvernementales • Isabelle Gusse

Au cœur des débats. Les grandes conférences publiques du prix Gérard-Parizeau 2000-2010 • Marie-Hélène Parizeau (dir.)

Les Autochtones et le Québec. Des premiers contacts au Plan Nord • Alain Beaulieu, Stéphan Gervais et Martin Papillon

Aux origines sociales de l'État-providence $\bullet$ Dominique Marshall

Aux sciences citoyen! Expériences et méthodes de consultation sur les enjeux scientifiques de notre temps $\bullet$ Marie-France Duranceau, Léonore Pion et Florence Piron

La bataille de l'imprimé. Àl'ère du papier électronique • Éric Le Ray et Jean-Paul Lafrance (dir.)

La Beauce Inc. Capital social et capitalisme régional • Jacques Palard

De la beauté comme violence. L'esthétique du fascisme français, 1919-1939• Michel Lacroix

Le bon sens à la scandinave. Les inégalités sociales de santé • Dominique Côté et Marie-France Raynault

La bourgeoisie industrielle au Québec • Arnaud Sales

Le Canada et l'Arctique • Franklyn Griffith, Rob Huebert et P. Whitney Lackenbauer

Le Canadien de Montréal. Une légende repensée • Audrey Laurin-Lamothe et Nicolas Moreau (dir.)

La capacité de choisir. Le Canada dans une nouvelle Amérique du Nord. George Hoberg (dir.)

La Chine imaginaire. Les Chinois vus par les Occidentaux, de Marco Polo à nos jours - Jonathan D. Spence

Le comportement électoral des Québécois • Éric Bélanger et Richard Nadeau

Consolidation de la paix et fragilité étatique. L'ONU en République Centrafricaine• Jocelyn Coulon et Damien Larramendy, Marie-Joëlle Zahar (coll.) 
Continuité et rupture. Les sciences sociales au Québec • Georges-Henri Lévesque (dir.)

Contre la réforme. La dérive idéologique du système d'éducation québécois • Normand Baillargeon

Converties à l'islam. Parcours de femmes au Québec et en France• Géraldine Mossière

Croissance et développement de l'enfant québécois de la naissance à six ans • Arto Demirjian

Le débat linguistique au Québec • Donat J. Taddeo et Raymond C. Taras

Décentralisation, politiques publiques et relations de pouvoir • Vincent Lemieux

Les défis du nationalisme moderne. Québec, Catalogne, Écosse • Michael Keating

La délinquance sexuelle des mineurs. Approches cliniques • Monique Tardif,

Martine Jacob, Robert Quenneville et Jean Proulx (dir.)

Démocratie médiatique et représentation politique • Denis Monière

La démographie québécoise. Enjeux du $\mathrm{xx}^{e}$ siècle • Victor Piché et Céline Le Bourdais (dir.)

Le désengagement de l'État providence $\bullet$ Nicole F. Bernier

Développement durable et participation publique. De la contestation écologiste aux défis de la gouvernance • Corinne Gendron et Jean-Guy Vaillancour (dir.)

La différenciation sociale. Modèles et processus • Danielle Juteau (dir.)

Un drame de la Deuxième Guerre. Le sort de la minorité japonaise aux États-Unis et au Canada • Greg Robinson

Douceville en Québec. La modernisation d'une tradition • Colette Moreux

Drogue et criminalité. Une relation complexe ( $2^{e}$ édition) $•$ Serge Brochu

Du pain ou du sang. Les travailleurs irlandais et le canal Beauharnois • Roland Viau

Éducation en milieu urbain $•$ Manuel Crespo et Claude Lessard (dir.)

De l'éducation spécialisée • Maurice Capul et Michel Lemay

Éduquer avant l'école. L'intervention préscolaire en milieux défavorisés et pluriethniques • François Victor Tochon (dir.)

Emploi et salaire. Deuxième édition revue et augmentée • Jean-Michel Cousineau

Énergie et fédéralisme au Canada • Michel Duquette

Les enfants qu'on n'a plus au Québec • Jacques Henripin, Paul-Marie Huot, Évelyne Lapierre-Adamcyk et Nicole Marcil-Gratton

L'énigme haïtienne. Échec de l_État moderne en Haïti • Sauveur Pierre Étienne

L'état des citoyennetés. En Europe et dans les Amériques • Jane Jenson, Bérengère Marques-Pereira et Éric Remacle (dir.)

L'éthique et le droit. Face aux nouvelles technologies biomédicales • Guy Bourgeault

L'ethnicité et ses frontières $•$ Danielle Juteau

Et si nous dansions? Pour une politique du bien commun au Canada • Charles Blattberg

L'Europe qui se fait. Regards croisés sur un parcours inachevé • Gérard Boismenu et Isabelle Petit (dir.)

Les excuses dans la diplomatie américaine. Pour une approche pluraliste des relations internationales $•$ Jérémie Cornut

La famille et les troubles émotionnels des jeunes • Claude Villeneuve

Le fédéralisme canadien contemporain. Fondements, traditions, institutions • Alain-G. Gagnon (dir.) 
Fédéralisme et cours suprêmes. Federalism and Supreme Courts • Edmond Orban et collaborateurs

Femmes philanthropes. Catholiques, protestantes et juives dans les organisations caritatives au Québec • Yolande Cohen

France-Canada-Québec. 400 ans de relations d'exception $\bullet$ Serge Joyal et Paul-André Linteau (dir.)

La France depuis de Gaulle. La Ve République en perspective • Marc Chevrier et Isabelle Gusse (dir.)

La gauche et la droite. Un débat sans frontières • Alain Noël et Jean-Philippe Thérien

Gérer l'environnement. Défis constants, solutions incertaines •

Edward A. Parson (dir.)

Gérer les catastrophes. L'incertitude à apprivoiser • Hélène Denis

Gestion des ressources humaines. Du modèle traditionnel au modèle renouvelé • Gilles Guérin et Thierry Wils

La gestion écologique des déchets • Jean-Guy Vaillancourt, Michel Séguin, Louis Maheu et Liliane Cotnoir, collaborateur: Pierre Hamel

La gestion environnementale et la norme ISO 14001 • Corinne Gendron

Les grandes universités de recherche. Institutions autonomes dans un environnement concurrentiel $\bullet$ Robert Lacroix et Louis Maheu

Grands projets et innovations technologiques au Canada $\bullet$ Philippe Faucher (dir.)

Grands Seigneurs et multinationales. L'économie politique de l'éthanol au Brésil • Michel Duquette

Les grèves au Canada: causes et conséquences • Robert Lacroix

Guide méthodologique universitaire. Un programme en 12 semaines • Aude Jimenez et Jamal-Eddine Tadlaoui

Gunnar Myrdal et son œuvre • Gilles Dostaler, Diane Éthier et Laurent Lepage (dir.)

Guy Rocher. Le savant et le politique • Violaine Lemay et Karim Benyekhlef (dir.)

Images et sociétés. Le progrès, les médias et la guerre • Catherine Saouter

Immigration et diversité à l'école. Le débat québécois dans une perspective comparative $\bullet$ Marie Mc Andrew

L'Inde et ses avatars. Pluralité d'une puissance • Serge Granger, Christophe Jaffrelot, Karine Bates et Mathieu Boisvert

L'individu et le citoyen dans la société moderne • Maryse Potvin, Bernard Fournier et Yves Couture (dir.)

Les inégalités sociales de santé au Québec • Katherine Frohlich, Maria

De Koninck, Andrée Demers et Paul Bernard (dir.)

Initiation à la recherche en géographie. Aménagement, développement territorial, environnement • Hervé Gumuchian et Claude Marois

L'intégration des familles d'origine immigrante. Les enjeux sociosanitaires et scolaires • Fasal Kanouté et Gina Lafortune (dir.)

L'intégration des immigrants. Cinquante ans d'action publique locale • Aude-Claire Fourot

L'interculturel au Québec. Rencontres historiques et enjeux politiques•

Bob W. White et Lomomba Emongo

Interprétations radicales $\bullet$ Daniel Laurier 
Introduction à la politique africaine. Deuxième édition revue et augmentée • Mamoudou Gazibo

Introduction à la victimologie • Jo-Anne Wemmers

Introduction aux relations internationales. Quatrième édition • Diane Éthier

Le Japon au travail • Bernard Bernier avec la collaboration de Vincent Mirza

Le Japon contemporain. Une économie nationale, une économie morale •

Bernard Bernier

Jeux de mémoire • Bruno Roy et Paul Zumthor (dir.)

Les Juifs du Maghreb. Diasporas contemporaines • Jean-Claude Lasry et Claude Tapia

Maintenir la paix en zones postconflit. Les nouveaux visages de la police •

Samuel Tanner et Benoit Dupont (dir.)

Les majorités fragiles et l'éducation. Belgique, Catalogne, Irlande du Nord, Québec • Marie Mc Andrew

Méchante langue. La légitimité linguistique du français parlé au Québec •

Chantal Bouchard

Méthodes de recherche pour l'éducation • Jean-Marie Van der Maren

Micro-économie. Exercices et corrigés • François Leroux

Milton Friedman et son cuvre • Marc Lavoie et Mario Seccareccia (dir.)

Les meurtriers sexuels. Analyse comparative et nouvelles perspectives • Jean Proulx, Maurice Cusson, Eric Beauregard et Alexandre Nicole (dir.)

Un modèle québécois? Gouvernance et participation dans la gestion publique • Pierre Hamel et Bernard Jouve

Les migrations internationales contemporaines. Une dynamique complexe au cour de la globalisation • François Crépeau, Delphine Nakache et Idil Atak (dir.)

Modernité et criminogenèse • Luigi Lombardi Vallauri (dir.)

Monuments intellectuels de la Nouvelle-France et du Québec ancien. Aux origines d'une tradition culturelle $\bullet$ Claude Corbo (dir.)

Les mouvements sociaux • Antimo L. Farro

Naissance d'une populationé Les Français établis au Canada au XVII siècle • Hubert Charbonneau (dir.)

Nouvelles valeurs et gouvernance au Canada $\bullet$ Neil Nevitte (dir.)

Un nouvel ordre mondial made in China? • Mamoudou Gazibo

et Roromme Chantal

L'ordre et la fête $•$ Frédéric Diaz

Où va le modèle suédois? État-providence et protection sociale •

Lionel-Henri Groulx

Les paradoxes de la démocratisation en Afrique. Analyse institutionnelle et stratégique - Mamoudou Gazibo

Parcours éducatifs: construction de soi et transformation sociale • Paul Bélanger

La pauvreté. Quatre modèles sociaux en perspective • Sylvain Lefèvre,

Gérard Boismenu et Pascale Dufour

Penser l'international. Perspectives et contributions des sciences sociales •

François Crépeau et Jean-Philippe Thérien (dir.)

Les pièges de l'austérité • Pierre Paquette et Mario Seccareccia

Planifier et évaluer l'action sociale • Ricardo Zúñiga 
Politique internationale et défense au Canada et au Québec • Kim Richard Nossal, Stéphane Roussel et Stéphane Paquin

La politique comparée. Fondements, enjeux et approches théoriques • Mamoudou Gazibo et Jane Jenson

La politique économique du développement et les modèles d'équilibre général calculable • Bernard Decaluwé, André Martens et Luc Savard

La politique en questions • Professeurs de science politique de l'Université de Montréal

La politique internationale en questions $•$ Professeurs de science politique de l'Université de Montréal

Les populations amérindiennes et inuit du Canada. Aperçu démographique • Louise Normandeau et Victor Piché (dir.)

La population du Québec d'hier à demain • Jacques Henripin et Yves Martin (dir.)

Pour comprendre le nationalisme, au Québec et ailleurs • Denis Monière

Pour un nouvel art de vivre. Entretiens sur la vie, la santé, l'éthique biomédicale et

l'éducation • Daisaku Ikeda, René Simard et Guy Bourgeault

Pour un regard-monde $•$ Armand Mattelart

Pratiques innovantes auprès des jeunes en difficulté • Denis Lafortune,

Marie-Marthe Cousineau et Claudia Tremblay (dir.)

Prévention des toxicomanies. Aspects théoriques et méthodologiques • Pierre Brisson

Profession démographe $\bullet$ Victor Piché

Profession géographe • Rodolphe De Koninck

Profession sinologue $\bullet$ Charles Le Blanc

Profession sociologue $\bullet$ Marcel Fournier

Protection de l'enfant: échec? • Alice Parizeau

Protection sociale et fédéralisme. L'Europe dans le miroir de l'Amérique du Nord • Bruno Théret

La psychocriminologie. Apports psychanalytiques et applications cliniques •

Dianne Casoni et Louis Brunet

La psychologie des adolescents $\bullet$ Michael Claes

Le Québec en jeu. Comprendre les grands défis • Gérard Daigle (dir.), avec la collaboration de Guy Rocher.

Les Québécois aux urnes. Les partis, les médias et les citoyens en campagne • Éric Bélanger, Frédérick Bastien, François Gélineau

Qitdlarssuaq. L'histoire d'une migration polaire • Guy Mary-Rousselière

Questions de criminologie • Jean Poupart, Denis Lafortune et Samuel Tanner (dir.)

Qui a peur de l'État? Le Canada dans un monde aux stuctures polycentriques de pouvoir • Gordon Smith et Daniel Wolfish (dir.)

Radioscopie de l'information télévisée au Canada • Denis Monière et Julie Fortier

Les raisons fortes. Nature et signification de l'appui à la souveraineté du Québec • Gilles Gagné et Simon Langlois

La recherche sociale en environnement. Nouveaux paradigmes $\bullet$ Robert Tessier et Jean-Guy Vaillancourt (dir.)

La recomposition du politique • Louis Maheu et Arnaud Sales (dir.)

Les relations ethniques en question. Ce qui a changé depuis le 11 septembre • Jean Renaud, Linda Pietrantonio et Guy Bourgeault (dir.) 
Le régime monétaire canadien. Institutions, théories et politiques • Bernard Élie $L a$ résolution de conflits. Guide d'implantation et de pratiques $\bullet$ Nina Admo

Les sciences sociales de l'environnement. Analyses et pratiques • Brigitte Dumas,

Camille Raymond et Jean-Guy Vaillancourt (dir.)

Les scientifiques et la paix. La communauté scientifique internationale au cours des années 20 • Brigitte Schroeder-Gudehus

Sexes et pouvoir. La construction sociale des corps et des émotions • Marc Préjean

Les sextants de Pékin • Joanna Waley-Cohen

Signé Papineau. La correspondance d'un exilé • Yvan Lamonde

Socialisation et communication dans les jeux vidéo • Charles Perraton,

Magda Fusaro et Maude Bonenfant (dir.)

La societé efficiente. Pourquoi fait-il si bon vivre au Canada?• Joseph Heath.

Traduit de l'anglais par Jean Chapdelaine Gagnon

La sociocriminologie • Stéphane Leman-Langlois

La sociologie des populations • Victor Piché et Hubert Gérard (dir.)

Sociologie et société québécoise. Présences de Guy Rocher • Céline Saint-Pierre et Jean-Philippe Warren (dir.)

Sphères de surveillance $\bullet$ Stéphane Leman-Langlois (dir.)

Stratégies de recherche en sciences sociales. Applications à la gérontologie • Richard Lefrançois

Le système de santé québécois. Un modèle en transformation • Clermont Bégin, Pierre Bergeron, Pierre-Gerlier Forest et Vincent Lemieux (dir.)

Le système politique américain • Michel Fortmann et Pierre Martin (dir.)

Terrorisme et anti-terrorisme au Canada • Stéphane Leman-Langlois et Jean-Paul Brodeur (dir.)

Théories de la politique étrangère américaine. Auteurs, concepts et approches • Charles-Philippe David (dir.)

Le Tiers-Monde postcolonial. Espoirs et désenchantements • Maurice Demers, Patrick Dramé (dir.)

Tradition et innovation en Chine et au Japon • Charles Le Blanc et Alain Rocher (dir.)

Traité de criminologie empirique • Marc Le Blanc et Maurice Cusson (dir.)

Trajectoires de la neutralité • Valérie Amiraux, David Koussens (dir.)

Le travail au féminin. Analyse démographique de la discontinuité professionnelle des femmes au Canada • Marianne Kempeneers (dir.)

Trois espaces de protestation. France, Canada, Québec • Pascale Dufour

L'univers social des adolescents • Michel Claes

Le vert et le bleu. Identité québécoise et identité irlandaise au tournant du $\mathrm{XX}^{e}$ siècle - Simon Jolivet

Les Vietnamiens de Montréal • Louis-Jacques Dorais et Éric Richard

Les visages de la police. Pratiques et perceptions • Jean-Paul Brodeur

$Z$ pour Zombies • Bernard Perron, Antonio Dominguez Leiva et Samuel Archibald (dir.) 
«J'ai connu la tentation du cliché », avoue le philosophe Alain Badiou en entrevue. Nous voilà rassurés : même les plus grands risquent de tomber dans les poncifs ou, comme l'expliquait Platon, dans une vision cynique et pauvre du monde. Ce livre pose le problème de l'obscurantisme lié à l'absence d'accès aux connaissances.

Le lecteur est donc convié à une aventure de vérification. Des spécialistes internationaux de plusieurs domaines (santé publique, anthropologie, sociologie, histoire, économie) déconstruisent ici quelques idées reçues autour de thèmes très variés: sida, santé maternelle, reproductive et sexuelle, accès aux soins, offre de soins, environnement, nutrition ... Le pari consiste à mettre en lumière l'importance d'une argumentation critique nuancée en examinant des idées largement véhiculées, c'est-à-dire celles qui ont cours dans le grand public. Par sa dimension éclectique, cet ouvrage est aussi divertissant qu'instructif pour lutter contre les clichés néfastes au progrès des Nations.

Valéry Ridde est professeur agrégé de santé publique à l'École de santé publique de l'Université de Montréal, titulaire d'une chaire de recherche en santé publique appliquée des Instituts de recherche en santé du Canada (IRSC) et chercheur à l'Institut de recherche en santé publique de l'Université de Montréal (IRSPUM)

Fatoumata Ouattara est docteure en anthropologie sociale de l'École des hautes études en sciences (EHESS) de Marseille. Elle est chargée de recherche à l'Institut de recherche pour le développement (IRD) en France et ses travaux portent sur la santé de la reproduction et les catégories morales. 